Nicolaus Henke

\title{
Wettbewerbsvorteile durch Integration von Geschäftsaktivitäten
}


Nicolaus Henke

\section{Wettbewerbsvorteile durch Integration von Geschäftsaktivitäten}

Die Integration der Geschäftsaktivitäten der Unternehmung wird zunehmend entscheidend zur Schaffung und Stabilisierung von Wettbewerbsvorteilen. Dies gilt insbesondere angesichts völlig neuer Möglichkeiten durch die Informationstechnik, die die Integration ganzer Unternehmen und Märkte verändern kann. Dieser Beitrag entwickelt einen Bezugsrahmen, der als Denkund Analyseraster Integrationsentscheidungen strukturieren und bewertbar machen soll. Der Bezugsrahmen wird auf 35 praktische Fallbeispiele aus den USA angewendet und eine Typologie von Integrationsstrategien abgeleitet.

Nicolaus Henke wurde 1961 geboren. Er studierte in Münster, Fribourg und an der Harvard University. Er wurde 1988 Diplom-Kaufmann, 1990 Master of Public Administration und promovierte 1993 zum Dr. rer. pol. Seit 1991 ist er Unternehmensberater. 
Wettbewerbsvorteile durch Integration von Geschäftsaktivitäten Ein zeitablaufbezogener wettbewerbsstrategischer Analyseansatz unter besonderer Berücksichtigung des Einsatzes von Kommunikations- und Informationssystemen (KIS) 


\section{Schpiften zu Marketine und Manasement}

Herausgegebenvon Prof.Dr. Dr.h.c. Heribert Meffert

Band 26

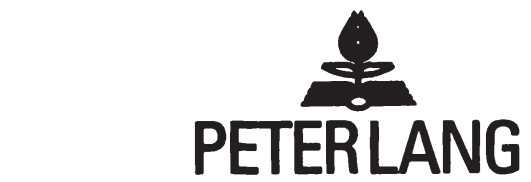

Frankfurtam Main · Berlin · Bern. NewYork. Paris.Wien 


\section{Nicolaus Henke}

\section{Wettbewerbsvorteile durch Integration von Geschäftsaktivitäten}

Einzeitablaufbezogener wettbewerbsstrategischerAnalyseansatz unter besonderer Berücksichtigung des Einsatzesvon Kommunikations- und Informationssystemen (KIS)

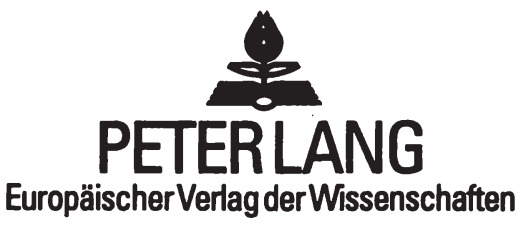


Die Deutsche Bibliothek - CIP-Einheitsaufnahme

Henke, Nicolaus:

Wettbewerbsvorteile durch Integration von Geschäftsaktivitäten : ein zeitablaufbezogener wettbewerbsstrategischer Analyseansatz unter besonderer Berücksichtigung des Einsatzes von Kommunikations- und Informationssystemen (KIS) / Nicolaus Henke. - Frankfurt am Main ; Berlin ; Bern ; New York ; Paris ; Wien : Lang, 1995

(Schriften zu Marketing und Management ; Bd. 26)

Zugl.: Münster (Westfalen), Univ., Diss., 1993

ISBN 3-631-49224-3

NE: GT

Open Access: The online version of this publication is published on www.peterlang.com and www.econstor.eu under the international Creative Commons License CC-BY 4.0. Learn more on how you can use and share this work: http://creativecommons.org/ licenses/by/4.0.

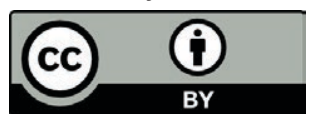

This book is available Open Access thanks to the kind support of ZBW - Leibniz-Informationszentrum Wirtschaft.

\author{
D 6 \\ ISSN 0176-2729 \\ ISBN 3-631-49224-3 \\ ISBN 978-3-631-75100-8 (eBook) \\ (c) Peter Lang GmbH \\ Europäischer Verlag der Wissenschaften \\ Frankfurt am Main 1995 \\ Alle Rechte vorbehalten.
}

Das Werk einschlieBlich aller seiner Teile ist urheberrechtlich geschützt. Jede Verwertung außerhalb der engen Grenzen des

Urheberrechtsgesetzes ist ohne Zustimmung des Verlages unzulässig und strafbar. Das gilt insbesondere für

Vervielfältigungen, Übersetzungen, Mikroverfilmungen und die Einspeicherung und Verarbeitung in elektronischen Systemen.

Printed in Germany 123467 


\section{Vorwort des Herausgebers}

Die Integration der Geschäftsaktivitäten stellt viele Unternehmen in einem von Diskontinuitäten, komplexeren Aufgaben sowie fortschreitender Technik geprägten Wettbewerbsumfeld vor neue Herausforderungen. Die rasante Entwicklung neuer Informationstechnologien bietet völlig neue Möglichkeiten zur Integration von Geschäftsaktivitäten, beispielsweise elektronische Netze zwischen Unternehmen oder die Abwicklung von Geschäften über elektronische Märkte. Damit stehen viele Unternehmen vor neuen Risiken und Chancen bei der Schaffung und Stabilisierung von Wettbewerbsvorteilen.

Die wissenschaftliche Diskussion der Integration von Geschäftsaktivitäten und ihre wettbewerbsstrategische Wirkung wurde in sehr unterschiedlichen Forschungsgebieten, u.a. der Organisationstheorie, der Institutionenökonomie, der Theorie der Wettbewerbsstrategie oder der Informationstechnik geführt. Auf den unterschiedlichen Integrationsbegriffen in der Literatur aufbauend hat sich der Verfasser die Aufgabe gestellt, einen Bezugsrahmen zur Ausgestaltung von Integrationsentscheidungen vorzulegen. Dabei steht die Frage im Mittelpunkt, welche Integrationsinstrumente die Unternehmung einsetzen kann und unter welchen Gegebenheiten deren Einsatz dauerhafte Wettbewerbsvorteile schaffen und absichern kann.

Ausgehend von allgemeinen Arbeiten zur Wirkung der Informationstechnik auf die Unternehmensstrategie und von unterschiedlichen Integrationsbegriffen in der organisationstheoretischen, informationstechnischen und institutionenökonomischen Literatur wird ein allgemeiner Bezugsrahmen zur Integration von Geschäftsaktivitäten entwickelt. Der Bezugsrahmen umfaßt die Strukturelemente einer Integrationsentscheidung. Seine Schwerpunkte liegen bei der Ableitung eines zeitablaufbezogenen Zielgerüsts, dem dauerhaften Wettbewerbsvorteil und einer Systematisierung der Integrationsinstrumente. 
Es werden drei Instrumente zur Integration von Geschäftsaktivitäten unterschieden, das Kommunikations- und Informationssystem, die Koordinationsmechanismen und die Kontrakte.

Eine empirische Fundierung des Bezugsrahmens erfolgt durch dessen Anwendung auf 35 Fallstudien. Dabei werden, abgeleitet aus dem Bezugsrahmen, sechs unterschiedliche Integrationsstrategien skizziert, die Transaktionsintegration, die Organisationsintegration, die Geschäftsprozeßintegration, vertikale Netzwerke, Information Partnerships und elektronische Märkte.

Die Arbeit stellt eine Bereicherung der wissenschaftlichen Diskussion u m die Schaffung und Erhaltung von Wettbewerbsvorteilen dar. Der Autor entwickelt unter sorgfältiger Auswertung der einschlägigen wissenschaftlichen Literatur, insbesondere der amerikanischen, ein systematisches Gerüst von Instrumenten zur Integration von Geschäftsaktivitäten und von Kriterien für deren Einsatz. Diese beruhen, gegenüber einer statischen Sicht, auf einem zeitablaufbezogenenen wettbewerbsstrategischen Grundmodell. Trotz der Schwierigkeit einer breiten empirischen Absicherung gelingt dem Autor ein Test des Ansatzes anhand einer explorativen Untersuchung von Fallstudien. Ferner werden wertvolle Anregungen für die weiterführende wissenschaftliche Diskussion und die praktische Planung abgeleitet. Es ist zu wünschen, daß die Untersuchung ein entsprechendes Echo in Wissenschaft und Praxis findet.

Prof. Dr. Dr. hc. H. Meffert 


\section{Vorwort des Verfassers}

Die Integration ist eine klassische Aufgabe des Managements einer arbeitsteilig in Geschäftsaktivitäten untergliederten Unternehmung. Sie wird auch in Betriebs- und Volkswirtschaftslehre seit langem diskutiert. Nur unvollständig gestellt (und beantwortet) sind jedoch zwei Fragen: erstens, wie Integration auf die Schaffung und Erhaltung des Wettbewerbsvorteils der Unternehmung wirkt, zweitens, welche neuen Möglichkeiten sich dabei durch den Einsatz neuer Informationstechniken ergeben.

Im Mittelpunkt der Arbeit steht ein Analyseansatz zur Entwicklung und Bewertung von Integrationsentscheidungen, insbesondere ein Gerüst von Integrationsinstrumenten und Vorteilhaftigkeitskriterien für deren Einsatz. Der Analyseansatz wird auf 35 Fallbeispiele angewendet, um generische Integrationsstrategien und deren wettbewerbsstrategische Wirkung abzuleiten.

Die Erstellung der Arbeit war nur mit vielfältiger Unterstützung verschiedener Personen möglich. Mein besonderer Dank gilt meinem Doktorvater, Herrn Professor Dr. Dr. h.c. Heribert Meffert. Er begleitete die Entwicklung der Themenstellung und die Fertigstellung der Arbeit intensiv, obwohl sie überwiegend in großer Entfernung zu Münster entstand, zunächst in Cambridge, Massachusetts, später parallel zur Berufstätigkeit in Deutschland. Für die jederzeitige Bereitschaft zu kritischen Diskussionen danke ich Herrn Prof. Dr. Martin Benkenstein.

Schließlich danke ich meinen Eltern und meiner lieben Ellen Kathrine für die jederzeitige Unterstützung und die mir von Ihnen gegebenene Unabhängigkeit, Kraft und Inspiration. 
Nicolaus Henke - 978-3-631-75100-8

Downloaded from PubFactory at 01/11/2019 08:21:33AM

via free access 
Inhaltsverzeichnis

Seite

A Gegenstand und wettbewerbsstrategische Bedeutung der Integration

1 Gegenstand der Integration von Geschäftsaktivitäten 3

1.1 Definition von Integration

1.2 Systematisierung der Integrationsinstrumente 10

2 Wettbewerbsstrategische Bedeutung der Integration 19

2.1 Wettbewerbsstrategische Bedeutung von Kommunikations- und Informationssystemen (KIS)

2.2 Wettbewerbsstrategische Bedeutung der Veränderung von Organisation und Unternehmensgrenze durch KIS

2.3 Wettbewerbsstrategische Bedeutung des Zwangs- und Bindungscharakters von Integrationsstrategien

3 Ziel und Gang der Arbeit 27

B Grundmodell und Bezugsrahmen zur wettbewerbsstrategischen Analyse von Integrationsentscheidungen

1 Gebundener Wettbewerb als Grundmodell

1.1 Bindungswirkung von Commitments 34

1.1.1 Starre Faktoren 35

1.1.2 Entgangene Optionen 37

1.1.3 Organisationale Trägheit 38 
1.2 Notwendigkeit zeitablaufbezogener Analyse und vorausschauender Planung

1.3 Dauerhafter Wettbewerbsvorteil als Zielgröße von Integrationsentscheidungen 44

1.3.1 Wettbewerbsposition .......................................................4 47

1.3.1.1 Qualitätsposition .............................................47

1.3.1.2 Kostenposition .............................................. 51

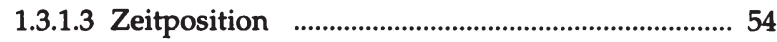

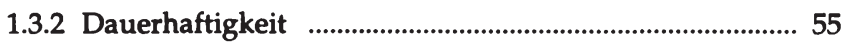

1.3.2.1 Stabilität ...................................................... 57

1.3.2.1.1 Abwehr von Imitation durch Mobilitätsbarrieren ............................. 58

1.3.2.1.2 Neue Regeln zur Umgehung von Mobilitätsbarrieren ...............................6 64

1.3.2.2 Flexibilität …................................................66

1.3.2.2.1 Erwartungswert der Aktionsflexibilität .68

1.3.2.2.2 Einflußfaktoren auf den Erwartungswert der Flexibilität 74

1.3.2.3 Verteilung 79

1.3.2.3.1 Abschöpfung …................................... 80

1.3.2.3.2 Nichtausnutzung ................................ 81 
2.1 Integrationsbereiche als Gegenstand der Integration 86

2.1.1 Mapping zur Bestimmung des Integrationsbereichs .... 88

2.1.2 Elemente des Integrationsbereichs und integrative Verbindungen 91

2.1.2.1 Horizontale Verflechtungen 92

2.1.2.2 Vertikale Verknüpfungen 96

2.2 Situative Bedingungen im Integrationsbereich 98

2.2.1 Volumen 100

2.2.2 Unsicherheit 102

2.2.3 Spezifität 105

2.3 Instrumente der Integration 107

2.3.1 Klassifikation der Integrationsinstrumente 108

2.3.1.1 Kommunikations- und Informationssystem (KIS) 108

2.3.1.1.1 Abwicklungssysteme 110

2.3.1.1.2 Management-Informationssysteme

2.3.1.1.3 Management-Unterstützungssysteme

2.3.1.1.3.1 Entscheidungs-Unterstützungssysteme 119

2.3.1.1.3.2 GruppenentscheidungsUnterstützungssysteme .... 121

2.3.1.1.3.3 Executive-Unterstützungssysteme 122

2.3.1.1.4 Expertensysteme 123

2.3.1.1.5 Büroautomatisierungssysteme 126 
2.3.1.2 Koordinationsmechanismen

2.3.1.2.1 Administrative Koordinationsmechanismen

2.3.1.2.2 Strukturelle Koordinationsmechanismen 131

2.3.1.2.3 Personale Koordinationsmechanismen 133

2.3.1.3 Kontrakte 135

2.3.1.3.1 Marktkontrakte 137

2.3.1.3.2 Unternehmenskonstituierende Kontrakte 139

2.3.1.3.3 Relationale Kontrakte 141

2.3.2 Integrationsinstrumente und situative Bedingungen 147

2.3.2.1 KIS und situative Bedingungen 147

2.3.2.2 Koordinationsmechanismen und situative Bedingungen 148

2.3.2.3 Kontrakte und situative Bedingungen 151

2.4 Nutzen und Kosten der Integration 153

2.4.1 Nutzen der Integration 154

2.4.2 Kosten der Integration 156

2.4.2.1 Autonomiekosten 157

2.4.2.2 Agencykosten 158

2.4.3 Optimaler Integrationsgrad 163

2.5 Zusammenfassende Darstellung des Bezugsrahmens 166 


\section{Wettbewerbsstrategische Wirkung von Integration}

- Explorative Untersuchung unter

besonderer Berücksichtigung von KIS -

1 Reichweiten der Integration und von Integrationsstrategien......169

1.1 Interne Integration .171

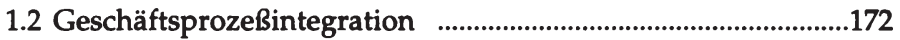

1.3 Geschäftsnetzwerkintegration ..............................................172

2 Explorative Untersuchung von Fallbeispielen

2.1 Interne Integration 178

2.1.1 Interne Integration bei Frito Lay, Inc. 178

2.1.1.1 Der Integrationsbereich: Auftragsprozeß und operative Steuerung

2.1.1.2 Einsatz von KIS im Integrationsbereich 181

2.1.2 Transaktionsintegration 186

2.1.3 Organisationsintegration 188

2.2 Geschäftsprozeßintegration 193

2.2.1 Geschäftsprozeßintegration bei Batterymarch 193

2.2.1.1 KIS-Einsatz im Geschäftsprozeß der Vermögensverwaltung .193

2.2.1.2 Dauerhafter Vorteil für Batterymarch? .196

2.2.2 Geschäftsprozeßintegration: Ansätze und Wirkungsweisen .198 
2.3 Geschäftsnetzwerkintegration ……………………………...... 206

2.3.1 Geschäftsnetzwerkintegration bei AHSC/Baxter ....... 207

2.3.1.1 KIS-Einsatz im Geschäftsnetzwerk Krankenhausbedarf 208

2.3.1.2 Kontraktuelle Integration zur Baxter Healthcare Corp. und die Folgen

2.3.2 Vertikale Netzwerke 217

2.3.2.1 Vertikale Netzwerke und Wettbewerbsposition

2.3.2.2 Stabilität vertikaler Netzwerke 220

2.3.2.3 Kooperative vertikale Netzwerke als Defensivstrategie 223

2.3.2.4 Dauerhaftigkeit in vertikalen Netzwerken: Zusammenfassung 224

2.3.3 Information Partnerships 227

2.3.3.1 Ansätze und Wirkungsweisen 228

2.3.3.2 Wettbewerbsposition und Dauerhaftigkeit .... 229

2.3.4 Elektronische Märkte 231

2.3.4.1 Integrationsbereich elektronischer Märkte .231

2.3.4.2 Wettbewerbsposition in elektronischen Märkten 235

2.3.4.3 Degeneration vertikaler Netzwerke zu elektronischen Märkten 236

2.3.4.4 Dauerhaftigkeit der Wettbewerbsposition in elektronischen Märkten 238

3 Einsatz von KIS zur Integration von Geschäftsaktivitäten: Ergebnisse der Fallstudienuntersuchung 240

D Zusammenfassung 


\section{Abbildungsverzeichnis}

Seite

Abb. A.1 Layers als Ebenen von Geschäftsaktivitäten ………………... 5

Abb. A.2 Unternehmensteile im Multiechelon-System f..................... 6

Abb. A.3 Kontrakte, Koordinationsmechanismen und KIS als

Strata

Abb. A.4 Beispiele für Informationstechnologien und KIS .............. 13

Abb. A.5 Beispiele für KIS in der Wertkette ....................................... 21

Abb. A.6 Bezugsrahmen mit Strukturelementen der Integrationsentscheidung …………………………………………........ 29

Abb. B.1.1 Stellung des Grundmodells im Bezugsrahmen ................. 32

Abb. B.1.2 Gründe für die Bindungswirkung von Commitments ... 35

Abb. B.1.3 Zahlungsprofil einer Strategie mit Kostenvorlauf ............ 36

Abb. B.1.4 Übergangswahrscheinlichkeiten zwischen Strategien ..... 42

Abb. B.1.5 Stellung des dauerhaften Wettbewerbsvorteils im Bezugsrahmen ………........................................................... 44

Abb. B.1.6 Einflußfaktoren auf den dauerhaften Wettbewerbsvorteil ......................................................................................... 46

Abb. B.1.7 Qualitätsbestimmende Faktoren ………................................. 48

Abb. B.1.8 Konsumenten- und Produzentenrente bei steigender Wettbewerbsintensität ............................................................... 57

Abb. B.1.9 Mobilitätsbarrieren als Quellen der Stabilität der Wettbewerbsposition ………………………………………..... 58

Abb. B.1.10 Strategic Gameboard ……………………................................. 64

Abb. B.1.11 Zahlungsprofile eines erfolgreichen (S) und eines nicht erfolgreichen (F) Szenarios ………………………………...... 68

Abb. B.1.12 Lernen und Wert der Flexibilität …………………………….. 76

Abb. B.1.13 Systematik des Verteilungsproblems ……………………..... 79 
Abb. B.1.14 Übersicht über die Einflußfaktoren auf den dauerhaften Wettbewerbsvorteil

Abb. B.2.1 Stellung des Integrationsbereichs im Bezugsrahmen ....... 87

Abb. B.2.2 Map eines Produktinnovationsprozesses $\quad$............................. 90

Abb. B.2.3 Materielle und immaterielle horizontale Verflechtungen

Abb. B.2.4 Auftragsprozeß Herstellung und Distribution eines Markenartikels 97

Abb. B.2.5 Stellung der situativen Bedingungen im Bezugsrahmen 99

Abb. B.2.6 Faktorstarrheit, -spezifität und -fungibilität 105

Abb. B.2.7 Situative Bedingungen im Integrationsbereich 107

Abb. B.2.8 Stellung der Integrationsinstrumente im Bezugsrahmen 108

Abb. B.2.9 Klassifikation von KIS …............................................. 110

Abb. B.2.10 Traditionelle Zuordnung von Anwendungsebenen ..... 112

Abb. B.2.11 Ablauf eines Bestellvorganges ohne Abwicklungssystem

Abb. B.2.12 Ablauf eines Bestellvorganges mit gemeinsamer Datenbank

Abb. B.2.13 Serielle Beziehung zwischen Abwicklungssystem und Management-Informationssystem 115

Abb. B.2.14 Übersicht über KIS bei semi-strukturierter Problemstruktur 118

Abb. B.2.15 Matrix von GruppenentscheidungsUnterstützungssystemen

Abb. B.2.16 Wichtigste Schritte bei der Definition eines ExecutiveUnterstützungssystems

Abb. B.2.17 Tradeoff zwischen Niveau und Einsetzbarkeit von Expertensystemen 
Abb. B.2.18 Ausgewählte Klassifikationen von Koordinationsmechanismen

Abb. B.2.19 Charakterisierung von marktbezogenen Kontrakten .... 138

Abb. B.2.20 Charakterisierung von unternehmenskonstituierenden Kontrakten

Abb. B.2.21 Systematik relationaler Kontrakte

Abb. B.2.22 Profil des Einsatzes von Koordinationsmechanismen in Abhängigkeit von der Unsicherheit der Aufgabe

Abb. B.2.23

Spezifität als Bestimmungsfaktor der Kontraktgestaltung

Abb. B.2.24 Stellung von Nutzen und Kosten im Bezugsrahmen .... 153

Abb. B.2.25 Nutzen der Integration 155

Abb. B.2.26 Kosten der Integration 162

Abb. B.2.27 Optimale Integration 163

Abb. B.2.28

Steigender optimaler Integrationsgrad durch KISEinsatz

Abb. B.2.29 Bezugsrahmen der Integrationsentscheidung 167

Abb. C.1.1 Fünf Ebenen der IT-induzierten Rekonfiguration von Unternehmungen 170

Abb. C.1.2 Systematisierung von Integrationsstrategien bei Einsatz von KIS

Abb. C.1.3 Unterscheidung der Integrationsstrategien anhand der Strukturelemente des Bezugsrahmens

Abb. C.2.1 Frito Lay, Inc.: Map des Integrationsbereichs Auftragsabwicklung und operative Steuerung

Abb. C.2.2 Frito Lay, Inc.: Ausschnitt aus den KIS-gestützten integrativen Verbindungen

Abb. C.2.3 Transaktionsintegration-COSMOS System bei ESAB .. 186

Abb. C.2.4 Überblick des Managementprozeß bei Xerox Corp. 189 
Abb. C.2.5 Geschäftsprozeßintegration bei Batterymarch Financial Management

Abb.C.2.6 Imitation von ATMs als Gefangenendilemma ............... 202

Abb. C.2.7 McGraw-Hill, Inc. als Informationsraffinierie ………….... 205

Abb. C.2.8 Integrationsbereich Distribution bei American

Hospital Supply Corporation .............................................. 209

Abb. C.2.9 Distribution bei American Hospital Supply

Corporation bis incl. ASAP 4 210

Abb. C.2.10 Geschäftssystem Krankenhausbedarf USA, März 1985 .. 213

Abb. C.2.11 Geschäftssystem Krankenhausbedarf USA, Ende $1985 \quad$.. 214

Abb. C.2.12 Benetton S.p.A.: Saisonzyklus ……………………………... 220

Abb. C.2.13 Evolutionsmuster von Strategien der elektronischen

Integration

Abb. C.2.14 Horizontale Verflechtungen im Reisemarkt ....................... 228

Abb. C.2.15 Aucnet elektronischer Gebrauchtwagenmarkt ................... 232

Abb.C.2.16 Degeneration vertikaler Netzwerke zu quasi

elektronischen Märkten als mehrperiodiges

Gefangenendilemma am Beispiel der Flug-

reservierungssysteme

Abb. C.3.1 Wettbewerbsstrategische Wirkung der

Integrationsstrategien 


\section{Abkürzungsverzeichnis ${ }^{1}$}

a. a. O. am angegebenen Ort

Abb. Abbildung

AHSC American Hospital Supply Corporation

allg. allgemein

Art. Artikel

ASAP Analytical Systems Automatic Purchasing

ATM Automatic Teller Machine

Aufl. Auflage

Bd. Band

bspw. beispielsweise

bzw. beziehungsweise

c. p. ceteris paribus

ca. circa

CAD Computer Aided Design

CAM Computer Aided Manufacturing

CEO Chief Executive Officer

COM Corporate Office Management

CSCW Computer Support of Cooperative Work

d. h. das heißt

Diss. Dissertation

DM Deutsche Mark

DSS Decision Support Systems

ed. Edition

EDI Electronic Data Interchange

EDV Elektronische Datenverarbeitung

ESS Executive Support Systems

et al. et alteri

etc. et cetera

1 Variablen einzelner Modelle sind jeweils im Text verzeichnet 
f, ff folgend, folgende

FN Fußnote

GDSS Group Decision Support Systems

GE Geldeinheiten

ggf. gegebenenfalls

H. Heft

Hrsg. Herausgeber

i. e. S. im engeren Sinne

i. d. R. in der Regel

i. S. im Sinne

insbes. insbesondere

IOS Interorganizational Systems

IS Informationssystem

Iss. Issue

IT Informationstechnologie

Jg. Jahrgang

Kap. Kapitel

KIS Kommunikations- und Informationssystem

m. a. W. mit anderen Worten

ME Mengeneinheiten

MIS Management-Informationssystem

No. Number

Nr. Nummer

NYCE New York Cash Exchange

o. J. ohne Jahrgang

o. O. ohne Ort

o. S. ohne Seite

o. V. ohne Verfasser

p. a. per anno

PPS Produktionsplanung- und Steuerung

rd. rund

ROE Return on Equity

ROI Return on Investment 
S. Seite

sog. sogenannte

Sp. Spalte

T Planungshorizont

t Zeitpunkt

Tab. Tabelle

u. ä. und ähnliches

u. a. und andere

u. a. O. und andere Orte

USA United States of America

US \$ United States Dollar

u. U. unter Umständen

vgl. vergleiche

Vol. Volume

WISU Das Wirtschaftsstudium

z. B. zum Beispiel

z. T. zum Teil

ZE Zeiteinheiten 
Nicolaus Henke - 978-3-631-75100-8

Downloaded from PubFactory at 01/11/2019 08:21:33AM

via free access 
A Gegenstand und wettbewerbsstrategische Bedeutung der Integration

Zunehmende Turbulenz und Dynamik der Umwelt sowie steigende Komplexität, Internationalisierung, Spezialisierung und Fragmentierung kennzeichnen den Wettbewerb in vielen Märkten. ${ }^{1} \mathrm{Um}$ in dieser Situation bestehen zu können, muß die Unternehmung ihre Leistungsfähigkeit nicht nur in den einzelnen zueinander interdependenten Geschäftsaktivitäten ${ }^{2}$ steigern. Um Wettbewerbsvorteile zu realisieren, ist auch die Verbindung der Geschäftsaktivitäten zu einem integrativen Ganzen (Integration) von entscheidender Bedeutung. ${ }^{3}$

Einerseits sind die Anforderungen an die Integration von Geschäftsaktivitäten gestiegen, angesichts einer zunehmenden Fragmentierung von Märkten mit einer Tendenz zu Mikromärkten4, einer immer gezielteren Segmentierung bzw. Disaggregation der Marketingstrategie - im Extremfall mit einem "Segment von Eins"5. Andererseits ergeben sich neue Inte-

1 Vgl. Meffert, H., Marketing und strategische Unternehmensführung - ein wettbewerbsorientierter Kontingenzansatz, in: Strategische Unternehmensplanung, Stand und Entwicklungstendenzen, (Hrsg.), Hahn, D., Taylor, B., Heidelberg, Wien, 1986, S. 660 ff; Szyperski, N., Führungstechnische Integration eines differenzierten Informations- und Kommunikationsmanagements, in: (Hrsg.), Seibt, D., Angewandte Informatik: Professor Dr. Paul Schmitz zu seinem 60. Geburtstag gewidmet, Braunschweig, 1985, S. 15 - 28, hier S. 23.

2 Der Begriff Geschäftsaktivität sei vorläufig umfassend definiert als Kombination von Elementarfaktoren oder Gruppen derselben (z.B. betriebswirtschaftliche Funktionen). Vgl. Gutenberg, E., Einführung in die Betriebswirtschaftslehre, Bd. 1, Die Produktion, Berlin, Göttingen, Heidelberg, 1951, S. 5; Meffert, H., Klassische Funktionenlehre und marktorientierte Führung - Integrationsperspektiven aus der Sicht des Marketing - in: Integration und Flexibilität, (Hrsg.), Albach, H. u.a., Wiesbaden, 1990, S. 372 - 406, hier: S. 375 ff. Zur Definition der Geschäftsaktivität vgl. Abschnitt A.1.1.

3 Vgl. Meffert, H., Marketing und strategische Unternehmensführung, a.a.O., S. 671; Porter, M., E., Competitive Advantage, New York, N.Y., 1985, S. 48; Porter, M.E., Millar, V.E., How information gives you competitive advantage, in: Harvard Business Review, Vol. 63, No. 4, 1985, S.149.

$4 \mathrm{Vgl}$. Meffert, H., Die Wertkette als Instrument einer integrierten Unternehmensplanung, in: Der Integrationsgedanke in der Betriebswirtschaftslehre, (Hrsg.), Delfmann, W., Kuhn, A., Wiesbaden, 1989, S. 274.

5 Vgl. Drucker, P. F., The Coming of the New Organization, in: Harvard Business Review, Vol. 66, 1988, S. 45 - 53; Lee, P.M., The Micro-Marketing Revolution, in: Small Business Reports, Vol. 15, 1990, No. 2, S. 71 - 82; Schiller, Z., Stalking the New Consumer, in: (Fortsetzung der Fußnote auf der nächsten Seite) 
grationsmöglichkeiten, vor allem aus neuen Kommunikations- und Informationssystemen (KIS), die auf neuen Informationstechnologien basieren.1 Die Bedeutung dieses Wandels für die Integration der Unternehmung wurde in der Literatur bereits früh erkannt. Leavitt und Whisler prognostizierten 1958 in ihrem klassischen Beitrag zur Informationstechnologie, daß die Organisation der Zukunft erheblich von der Integration mit KIS beeinflußt werden würde. ${ }^{2}$ Galbraith nannte bereits 1968 seinen Beitrag "Achieving Integration Through Information Systems"3 und betrachtete KIS als eine der Schlüsselvariablen der Unternehmensführung. ${ }^{4}$ Kubicek und Kieser wiesen 1975 auf das Potential von KIS hin, die Koordination der Unternehmung zu verändern. ${ }^{5}$ Aufgrund der weitreichenden Auswirkungen neuer Integrationsstrategien auf die Unternehmung, ihre Organisation und ihre Grenzen kann sich der Zuschnitt ganzer Unternehmungen oder Branchen erheblich verändern. ${ }^{6}$ Kernfrage dieser Arbeit ist es, ob und unter welchen allgemeinen Bedingungen die Schaffung dauerhafter Wettbewerbsvorteile durch die Integration von Geschäftsaktivitäten, unter besonderer Berücksichtigung des Einsatzes von KIS, möglich ist.

Business Week, 26. August 1989, S. 54 - 62; Osborn, T.W., Micro Marketing into the Niches: The Eye of the Stranger, in: Marketing Communications, Vol. 12, 1987, No. 3, S. 57 - 72; Webber, J.C., Packaged Goods Marketing Research - Where's It All Going, in: Journal of Advertizing Research, Vol. 26, 1986, No. 5, S. RC3 - RC5.

1 Vgl. Zuboff, S., In the Age of the Smart Machine, New York, N.Y., 1988, S. 415.

2 Vgl. Leavitt, Th., Whisler, Th.L., Management in the 1980's, in: Harvard Business Review, Vol. 36, 1958, S. 41 ff; Whisler, Th.L., Information Technology and Organizational Change, Belmont, California, 1970.

3 Galbraith, J.R., Achieving Integration Through Information Systems, in: Academy of Management Proceedings, 12, 1968, S. 111.

4 Galbraith, J.R., Designing Complex Organizations, Reading, Massachusetts u.a.O., 1973, S. 17 sowie Kapitel 4. Demgegenüber wurde Informationstechnologie in der deutschen Organisationsliteratur zwar als Parameter erkannt, jedoch überwiegend nicht als zu gestaltende Instrumentalvariable, sondem als kontextbestimmtes situatives Datum einbezogen. Vgl. z.B. Kieser, A., Kubicek, H., Organisation, Berlin, 1983, S. 291 ff und dort angegebene Literatur. Aus einer solchen Perspektive kann zwar der EinfluB der Informationstechnologie z.B. auf die Spezialisierung oder die Zentralisation bzw. Dezentralisation diskutiert werden, nicht aber die Frage, wann Informationstechnologie zu welchen Integrationsaufgaben eingesetzt werden sollte.

5 Vgl. Kieser, A., Kubicek, H., An Organizational Concept for the Design of Management Information Systems, in: Information Systems and Organisational Structure, (Hrsg.), Grochla, E., Szyperski, N., Berlin, New York, 1975, S. 166; Kubicek, H., Informationstechnologie und organisatorische Regelungen, Berlin, 1975, S. 240 ff.

6 Vgl. Rockart, J.F., Short, J.E., IT in the 1990's: Managing Organizational (Fortsetzung der Fußnote auf der nächsten Seite) 
In der Literatur liegt bisher kein eindeutiger begrifflicher Rahmen für die Integration von Geschäftsaktivitäten vor. Ein solcher ist im folgenden $\mathrm{zu}$ erarbeiten, unter Bezugnahme auf Anhaltspunkte, die sich aus der Literatur ergeben.

\subsection{Definition von Integration}

Integration konstituiert ein System als Ganzes. ${ }^{1}$ Das hier betrachtete System ist die Unternehmung. Die Integration richtet sich auf die Verbindung der Geschäftsaktivitäten der Unternehmung. Die Träger der Geschäftsaktivitäten sind die Unternehmensteile. Als Unternehmensteile

Interdependence, in: Sloan Management Review, Vol. 30, Winter 1989, S. 7 - 17

1 Vgl. Ulrich, H., Integrative Unternehmensführung, in: Kirsch, W., Picot, A., Die Betriebswirtschaftslehre im Spannungsfeld zwischen Generalisierung und Spezialisierung: Wiesbaden, 1989, S. 185; Lehmann, H., Integration, in: Handwörterbuch der Organisation, (Hrsg.), Grochla, E., 2. Aufl., Stuttgart, 1980, Sp. 976 f; Kosiol, E., Einführung in die Betriebswirtschaftslehre, Wiesbaden, 1968, S. 83 ff; Schweiker, K., Grundlagen einer Theorie betrieblicher Datenverarbeitung, Wiesbaden, 1966, S. 124; Heinen, E., Industriebetriebslehre als Entscheidungslehre, in: Industriebetriebslehre, Entscheidungen im Industriebetrieb, (Hrsg.), Heinen, E., Wiesbaden, 1978, S. 25. Etymologisch kann der Begriff der Integration zurückgeführt werden auf lat. integrare (wiederherstellen, erneuern), integratio (Emeuerung) und integer (ganz, unberührt). Integration von zuvor unabhängigen Elementen konstituiert insofern ein neues Ganzes. Vgl. Lehmann, H., Integration, a.a.O., Sp. 977. Die Verbindung von Elementen zu einem Ganzen bedeutet dabei nicht deren Verschmelzung, aber die Bildung eines organischen

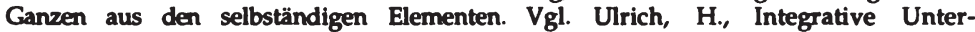
nehmensführung , a.a.O., S. 185. Die Frage der Integration selbst ist alles andere als neu, vielmehr ein seit langem diskutiertes Thema in mehreren Disziplinen, etwa in der Soziologie als soziale Integration angesichts gesellschaftlicher Differenzierung: Spencer, H., System of Synthetic Philosophy, Aberdeen, 1893, S. 265, in der Volkswirtschaftslehre als Abbau von Handelshemmnissen und Eingliederung von Volkswirtschaften in supranationale Organisationen: 2.B. Balassa, B., The theory of economic integration, Homewood, Illinois, 1961; Sannwald, R., Wirtschaftliche Integration, Basel, 1958, in der Organisationspsychologie im Hinblick auf die Eingliederung von Individuen in soziale Kontexte: vgl. Jung C.G., Integration of the personality, New York, 1939; Argyris, Ch., Integrating the Individual and the Organization, New York, 1964. Auf eine nähere Diskussion dieser Integrationsbegriffe sei verzichtet, da dies zur Lösung der Frage dieser Arbeit wenig beiträgt. 
seien allgemein Teilmengen der Unternehmung bezeichnet, also einzelne Mitarbeiter, Gruppen, Abteilungen oder Unternehmensbereiche. Geschäftsaktivitäten selbst sind zwar Ausgangspunkt der Betrachtung, jedoch nicht die zu verbindenden Elemente. Vielmehr sind Unternehmensteile als Träger der Geschäftsaktivitäten die materiellen Objekte der Integration, selbst wenn die Integration auf die Geschäftsaktivitäten gerichtet ist.1

Die Elemente des zu integrierenden Systems Unternehmung können nach verschiedenen Dimensionen geordnet werden. Dem systemtheoretischen Multilevel-Ansatz folgend, seien drei Ordnungen (Dimensionen von Ebenen) in einem System unterschieden: Layers, Echelons und Strata. ${ }^{2}$ Die zentralen Begriffe dieser Arbeit können aus diesen Ordnungen abgeleitet werden: Geschäftsaktivitäten beziehen sich auf Layers, Unternehmensteile auf Echelons und Integrationsinstrumente auf Strata.

Geschäftsaktivitäten sind die wertschöpfende Umwandlung von Produktionsfaktoren zur Lösung eines Kundenproblems. Damit können sehr verschiedene Aggregationsniveaus gemeint sein: Die Kombination von Elementarfaktoren, dispositiven Tätigkeiten oder Gruppen derselben (z.B. Funktionen, Geschäftsfelder). ${ }^{3}$ Damit entsprechen sie den "Layers" des Multilevel-Ansatzes. Jedes Layer ist eine Meta-Ebene des vorstehenden Layers. Allgemein unterscheiden Mesarovic et al. die Layers Process, Selection, Learning and Self-Organisation. Integration richtet sich auf die Verbindung der Geschäftsaktivitäten der Unternehmung. Dabei ist anzu-

1 Lehmann, H., Integration, a.a.O., Sp. 978 ff unterscheidet diesbezüglich immaterielle Integrationsobjekte (z.B. Ziele) und materielle Integrationsobjekte (z.B. Menschen, Maschinen).

2 Mesarovic, M.D., Macko, D., Takahara, Y., Theory of Hierarchical, Multilevel Systems, New York, London, 1970, S. 37. Diese Begriffe könnten jeweils als "Schicht" oder "Ebene" übersetzt werden, sie werden zur Beibehaltung der Eindeutigkeit nicht übersetzt.

3 Vgl. Gutenberg, E., Betriebswirtschaftslehre, a.a.O., S. 5; Meffert, H., Klassische Funktionenlehre, a.a.O., S. 375: Elementarkombinationen von Produktionsfaktoren und dispositiven Tätigkeiten. Porter unterscheidet primäre und sekundäre Wertaktivitäten dies ist ein allgemeinerer Ansatz, der auch höhere Metaebenen bzw. Gruppen von Geschäftsaktivitäten als Geschäftsaktivitäten definiert Porter, M.E., Competitive Advantage, a.a.O., S. 39. 
geben, auf welcher Ebene Geschäftsaktivitäten integriert werden (sollen). In dem in Abb. A.1 gewählten Beispiel wird unterschieden die Integration der Geschäftsaktivitäten des Produktionsprozesses, der einzelnen Aktivitäten der Produktionsprogrammplanung, der Produktentwicklung und der Produktinnovation und -positionierung. ${ }^{1}$

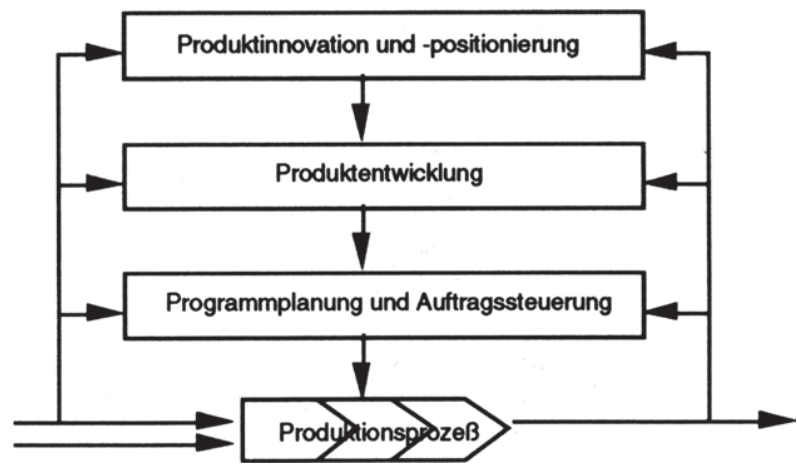

Abb. A.1: Layers als Ebenen von Geschätsaktivitäten

Darstellung nach Mesarovic, M.D., Macko, D., Takahara, Y., Multilevel Systems, a.a.O., S. 37.

Jedes der übergeordneten Layers kann dabei zwar als verwandtes bzw. als Meta-Problem des nachgeordneten angesehen werden, es hat jedoch eine andere Problemstruktur, unterschiedliche Abläufe von Aktivitäten, andere Beteiligte und andere Zeithorizonte. Folglich liegt für die Analyse der Integration der verschiedenen Layers eine völlig verschiedene Kette von Geschäftsaktivitäten vor. Eine sachgerechte Definition der zu betrachtenden Geschäftsaktivität(en) muß sich aus der Problemdiagnose im Einzelfall ergeben, d.h. Lagerhaltungsprobleme werden sich tendenziell aus dem Produktionsprozeß oder der Auftragssteuerung ergeben, die

1 Ein Integrationsproblem kann auch für zwei oder mehrere dieser Ebenen definiert werden, z.B. die Integration von Konstruktion und Produktion durch CAD/CAM. 
Senkung von Entwicklungskosten aus der Produktpositionierung und der Produktentwicklung.

Integration richtet sich auf die Verbindung von Geschäftsaktivitäten. Dabei muß allerdings an konkreten Integrationsobjekten angesetzt werden. Dies sind die Unternehmensteile. Im Multilevel-Ansatz entsprechen Unternehmensteile den Echelons. (vgl. Abb. A.2)

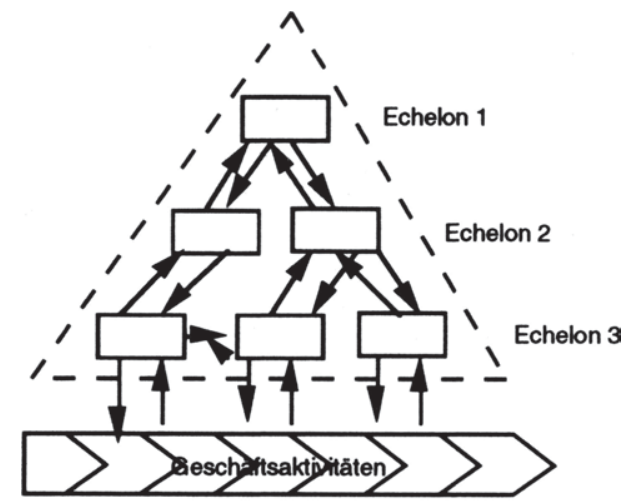

Abb. A.2: Untemehmensteile im Multiechelon-System

Darstellung nach Mesarovic, M.D., Macko, D.,

Takahara, Y., Multilevel Systems, a.a.O., S. 49.

Die Unternehmensteile sind aufbauorganisatorische Teilmengen, z.B. einzelne Mitarbeiter, Gruppen, Abteilungen oder Unternehmensbereiche. Sie sind die Träger der Geschäftsaktivitäten, wie auch in Abb. A.2 schematisch dargestellt.

Die Notwendigkeit, Geschäftsaktivitäten zu integrieren, resultiert aus Interdependenzen. ${ }^{1}$ Interdependenzen zwischen betrieblichen Entscheidungen bestehen, wenn die zielsetzungsgerechte Gestaltung einer Geschäfts-

1 Vgl. Adam, D., Kurzlehrbuch Planung, Wiesbaden, 1986, S. 50 ff. Als Interdependenzen werden hier die von Adam unterschiedenen sachlichen Kopplungen und (Fortsetzung der Fußnote auf der nächsten Seite) 
aktivität nicht unabhängig ist von der Gestaltung einer anderen Geschäftsaktivität. Thompson unterscheidet drei Arten von Interdependenzen: gepoolte, sequentielle und reziproke. ${ }^{1}$ Gepoolte Interdependenzen sind gegeben, wenn für verschiedene Geschäftsaktivitäten auf die gleichen Ressourcen zugegriffen wird. Sequentielle Interdependenzen liegen vor, wenn Geschäftsaktivitäten im Geschäftssystem derart nacheinandergelagert sind, daß die Gestaltung einer Geschäftsaktivität vom Output einer vorangegangenen Geschäftsaktivität (oder Informationen darüber) beeinflußt wird. Als reziprok bezeichnet Thompson Interdependenzen, wenn zwischen Unternehmensteilen gegenseitig sequentielle Interdependenzen vorliegen. ${ }^{2}$

Interdependenzen in der Unternehmung können demnach zwei Ursachen haben: Geschäftsaktivitäten können einander nachgelagert (sequentiell oder reziprok) sein oder auf gemeinsame Ressourcen zugreifen. $^{3}$ Dementsprechend werden zwei Arten integrativer Verbindungen unterschieden: vertikale Verknüpfungen und horizontale Verflechtungen. Eine Integration von im Geschäftssystem nacheinandergelagerten Geschäftsaktivitäten, die Schnittstellen zueinander aufweisen, wird als vertikale Verknüpfung bezeichnet. Die integrativen Verbindungen gleichartiger Geschäftsaktivitäten, die gemeinsam ausgeführt werden können oder gemeinsame Ressourcen nutzen, ${ }^{4}$ werden als horizontale Verflechtungen bezeichnet. Daraus ergibt sich die Definition:

Erfolgskopplungen zusammengefaßt.

1 Vgl. hierzu und zum folgenden Thompson, J.D., Organizations in Action, New York, 1967, S. 54 ff.

2 Ähnlich, jedoch abweichend von Thompson, unterscheidet Frese Entscheidungsinterdependenzen, Interdependenzen aufgrund innerbetrieblicher Leistungsverflechtung, Ressourceninterdependenzen und Marktinterdependenzen. Vgl. Frese, E., Grundlagen der Organisation, 4. Aufl., Wiesbaden, 1988, S. 195 ff, dessen Kritik an der Definition von Thompson für diese Arbeit jedoch nicht relevant ist.

3 Emery, J.C., Management Information Systems, New York, Oxford, 1987, S. 243 f.

4 Dabei sei der Begriff der gemeinsam genutzten Ressource sehr umfassend verstanden, nicht lediglich als Rohstoff, sondem auch als Zugang zu einem Markt, zu einer Produktionstechnologie, zu Management-know-how. 
Integration ist die horizontale Verflechtung und vertikale Verknüpfung von Unternehmensteilen. ${ }^{1}$

Diese Definition hat folgende Implikationen. Jede Unternehmung ist immer in einem bestimmten Umfang integriert, d.h. sie hat einen bestimmten Integrationsgrad. Der Integrationsgrad ist allgemein das Ausmaß, in dem Interdependenzen durch horizontale Verflechtungen und vertikale Verknüpfungen von Unternehmensteilen berücksichtigt werden. Der Integrationsgrad ist hoch, d.h. ein System ist vollkommen integriert, wenn alle Interdependenzen durch vertikale Verknüpfungen und horizontale Verflechtungen berücksichtigt sind. Der Umkehrfall, Nichtintegration, kann lediglich bei Unternehmensteilen auftreten, die in jeder Beziehung unverbunden sind. Dies ist innerhalb eines Unternehmens praktisch nicht denkbar. Neben der Integration von Teilen eines Unternehmens ist durchaus auch die Integration zwischen Unternehmensteilen unterschiedlicher Unternehmen möglich.

Die Begriffe "vertikal" und "horizontal" wurden den Begriffen Verknüpfung und Verflechtung beigegeben, um einerseits auf die eingeführten Begriffe Verknüpfung und Verflechtung zurückzugreifen, andererseits Eindeutigkeit zu gewährleisten. ${ }^{2}$ Die Begriffe "vertikal" und "horizontal" werden dabei wie in der Strategieliteratur ${ }^{3}$ verstanden. Eine davon ver-

1 Eine ausführlichere Charakterisierung dieser neu in die Literatur eingeführten Begriffe erfolgt in Abschnitt B.2.1.2.

2 Verknüpfungen (linkages) und Verflechtungen (interrelationships) werden als Einflußgröße ("Driver") in der strategischen Kosten- bzw. Differenzierungsanalyse benutzt. Linkages werden dabei gelegentlich auch als vertical linkages bezeichnet. Vgl. Porter, M.E., Competitive Advantage, a.a.O., S. 50 ff und 323 ff.

3 In der Literatur der Unternehmensstrategie wird regelmäBig als vertikale Integration die Abstimmung oder Einbeziehung von vor- oder nachgelagerten Stufen des Wertschöpfungsprozesses angesehen. Vgl. Chandler, A.D., Strategy and Structure: Chapters in the History of the American Enterprise, Cambridge, Massachusetts, London, 1962, S. 14; Porter, M.E., Competitive Advantage, a.a.O., S. 55; Koch, H., Integrierte Unternehmensplanung, Wiesbaden, 1982, S. 9. Bei Betrachtung der Ablauforganisation verwenden den Begriff in diesem Sinne auch Mintzberg, H., Structuring of Organizations, a.a.O., S. 406; Galbraith, J.R., Complex Organizations, a.a.O., S. 17. Dies deckt sich mit (Fortsetzung der Fußnote auf der nächsten Seite) 
schiedene Bedeutung liegt in der Organisationsliteratur ${ }^{1}$ vor. In beiden wird "vertikal" für nacheinandergelagerte, in einer Reihenfolge stehende Unternehmensteile verwendet, und "horizontal" für nebeneinanderstehende Unternehmensteile ohne eindeutige Beziehung zueinander. In der Organisationsliteratur bezieht sich dabei die Ordnung oder Reihung von Unternehmensteilen auf die Aufbauorganisation der Unternehmung, in der Strategieliteratur auf den geleisteten Geschäfts- bzw. Wertschöpfungsprozeß. Eine "vertikale Beziehung" ist demnach aus Sicht der Organisationsliteratur eine hierarchische Über-bzw. Unterordnung, in der Strategieliteratur die Relation z. B. von Produktion und Absatz. Keiner der beiden Ansätze ist richtig oder falsch. Für diese Arbeit erscheint die in der Strategieliteratur verwendete Terminologie zweckmäßiger.

Der entwickelte Integrationsbegriff wurde aus Anhaltspunkten der systemtheoretischen, der organisationstheoretischen und der betriebswirt-

dem Begriff der vertikalen Integration der volkswirtschaftlichen Wettbewerbstheorie und -politik und der Transaktionskostenökonomie. Vgl. Williamson, O.E., Economic Institutions, a.a.O., S. 85 ff. Demgegenüber wird als horizontale Integration die Abstimmung oder Einbeziehung nebeneinanderstehender, nicht eindeutig vor- oder nachgelagerter Stufen des Wertschöpfungsprozesses angesehen. Vgl. Koch, H., Integrierte Unternehmensplanung, a.a.O., S. 9; Porter, M.E., Competitive Strategy, a.a.O., S. 364 ff. Dies deckt sich emeut mit dem Begriff der horizontalen Konzentration der Wettbewerbstheorie, womit beispielsweise ein hoher Marktanteil oder eine starke Konzentration in einem Markt gemeint sind. Die starke Konzentration in einem Markt entsteht durch das Zusammenfügen gleichartiger, nicht im Wertschöpfungsprozeß hintereinandergelagerter Unternehmen.

1 In der Onganisationsliteratur treten die Begriffe "vertikal" und "horizontal" in Verbindung mit dem Problem der aufbauorganisatorischen Dezentralisierung und Koordination auf. Beispielsweise sieht Mintzberg es als vertikale Dezentralisierung an, wenn Entscheidungsrechte von der Unternehmensführung auf hierarchische untergeordnete Unternehmensteile delegiert werden. Demgegenüber wird es als horizontale Dezentralisierung angesehen, wenn Entscheidungsrechte auf gleicher hierarchischer Ebene oder in Stäbe delegiert werden. So definiert auch Frese in diesem Zusammenhang Koordination wie folgt: "Vertikale Koordination bedeutet Zerlegung und Zuondnumg von Entscheidungsvektoren und Sicherung der daraus resultierenden hierarchischen Kommunkationsbeziehungen. Horizontale Koordination vollzieht sich durch nichthierarchische (horizontale wie diagonale) Kommunikation." Frese, E., Koordination, in: Handwörterbuch der Betriebswirtschaft, (Hrsg.), Grochla, E., Wittmann, W., 4. Aufl., Stuttgart, 1975, Sp. 2266. Es wird ersichtlich, daß von vertikal gesprochen wird, wenn hierarchische Nach- oder Unterondnungsbeziehungen vorliegen, demgegenüber von horizontal, wemn Beziehungen zwischen hierarchisch nebeneinander stehenden Einheiten betrachtet werden. Vgl. Mintzberg, H., The Structuring of Organizations, Englewood Cliffs, New Jersey, 1979, S. 188; Schanz, G., Organisationsgestaltung, München, 1982, S. 135. 
schaftlichen Literatur abgeleitet. Aus der systemtheoretischen Perspektive ist Integration die Verbindung von materiellen oder immateriellen Elementen (Integrationsobjekten) zu einem System. Auf Basis des organisationstheoretischen Interdependenzbegriffs wurden die integrativen Verbindungen und Integrationsobjekte (Unternehmensteile) inhaltlich bestimmt. Unter Rückgriff auf die betriebswirtschaftliche Literatur wurde konkretisiert, worauf Integration zu richten ist: auf nacheinandergelagerte oder gleichartige Geschäftsaktivitäten in einem Geschäftssystem. Damit liegt eine Integrationsdefinition vor, die die abstrakte Systematik der systemtheoretischen und organisationstheoretischen Literatur mit dem Konkretisierungsniveau der betriebswirtschaftlichen Literatur verbindet. Offen bleibt jedoch in der Literatur wie in der Definition, auf welche Weise, d.h. mit welchen Instrumenten, die so definierte Integration von Geschäftsaktivitäten erreicht werden kann. ${ }^{1}$ Zur Klärung dieser Frage kann wiederum der Multilevel-Ansatz herangezogen werden. Die Integrationsinstrumente lassen sich als "Strata" systematisieren.

\subsection{Systematisierung der Integrationsinstrumente}

Strata sind inhaltlich verschiedene Aspekte des Gesamtsystems Unternehmung, ${ }^{2}$ d.h. inhaltlich verschiedene Betrachtungsebenen. Für die Integration der Unternehmung können drei Aspekte unterschieden werden, ${ }^{3}$ die

1 Ausnahmen: Galbraith, J.R., Complex Organizations, a.a.O., S. 15; Meffert, H., Klassische Funktionenlehre, a.a.O., S. $255 \mathrm{ff}$.

2 Mesarovic, M.D., Macko, D., Takahara, Y., Multilevel Systems, a.a.O., S. 4 ff. Zum Begriff des Aspekt-Systems vgl. auch Wouw, C., Information Systems Planning, Eindhoven, 1977, S. 15; Hübner, H., Informationsmanagement, Strategie - Gestaltung Instrumente, München, Wien, 1984, S. 93 ff.

3 Zum organisationalen und technischen Integrationsbegriff vgl. Grochla, E., Grundfragen der informationstechnologischen Integration, in: (Hrsg.), Seibt, D., Angewandte Informatik, a.a.O., S. 8. Die drei Integrationsbegriffe sind weder überschneidungsfrei noch voneinander unabhängig. Neben diesen Integrationsbegriffen wird in der verhaltenswissenschaftlichen Literatur noch die personale Integration diskutiert. Vgl. z.B. Argyris, Ch., Integrating the Individual, a.a.O. Des weiteren wird der Begriff Integration häufig für die Zusammenfügung von Theorien verwendet. Vgl. z.B. Scholz, Ch., Strategisches Management, a.a.O., der als "integrativen Ansatz" zur (Fortsetzung der Fußnote auf der nächsten Seite) 
sowohl verschiedene Gegenstandsbereiche und damit verschiedene Integrationsinstrumente bezeichnen: die (informations)-technische, die organisationale und die kontraktuelle Integration. Diese sind, in Anlehnung an Mesarovic, in Abb. A.3 als Strata dargestellt.

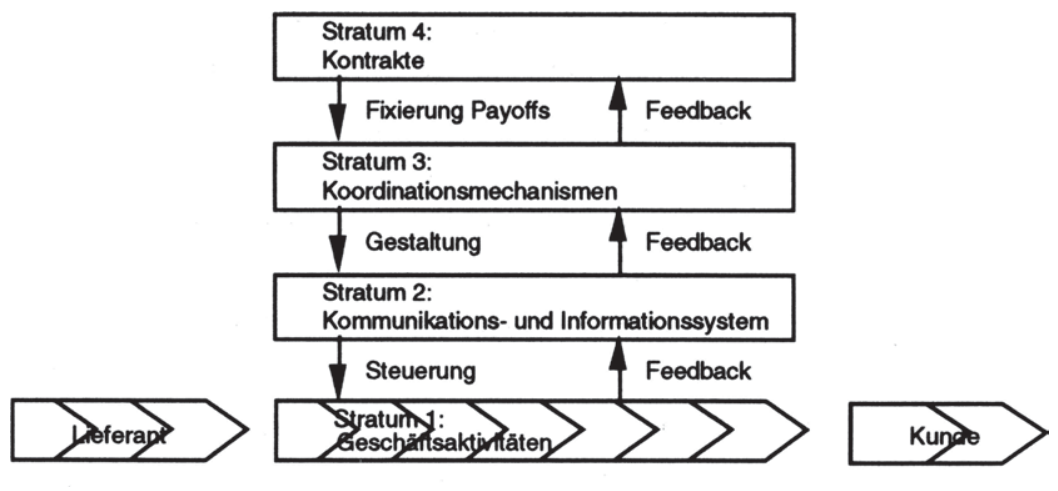

Abb. A.3: Kontrakte, Koordinationsmechanismen und KIS als Strata

Alle Strata sind auf das gleiche Problem gerichtet, nämlich auf die wertschöpfende Umwandlung von Produktionsfaktoren in die Lösung des Kundenproblems. Jedes Stratum betrachtet und beeinflußt den Wertschöpfungsprozeß jedoch auf verschiedene Weise, d.h. nach verschiedenen Aspekten. ${ }^{1}$ Jedes Stratum hat ein eigenes Muster von Begriffen, Konzepten oder Prinzipien.2 Für jedes Stratum kann Integration spezifisch

Unternehmensstrategie die Zusammenfügung von Konzepten der strategischen Unternehmensführung betrachtet. Busch, E.W., Integrationskonzepte der Betriebswirtschaftslehre: das Beispiel der Harvard Business School, in: Die Betriebswirtschaft, Vol. 50, S. 237 - 248 sieht die Fallstudienmethodik und das Curriculum der Harvard Business School als ein Integrationskonzept der Betriebswirtschaftslehre in der Lehre. Vgl. dazu auch Adam, D., Backhaus, K., Meffert, H., Wagner, H., (Hrsg.), Integration und Flexibilität. Eine Herausforderung für die Allgemeine Betriebswirtschaftslehre, Wiesbaden, 1989, S. 137 ff.

1 Die Strata werden unterschieden unabhängig von den Trägern der Entscheidungen (Unternehmensteilen) oder des jeweiligen Komplexitäts- und Abstraktionsniveaus (Geschäftsaktivitäten). Diese wenden durch die Dimensionen Echelons und Layers abgebildet.

2 Mesarovic, M.D., Macko, D., Takahara, Y., Multilevel Systems, a.a.O., S. 41. 
operationalisiert werden: Die kontraktuelle Integration durch die Gestaltung der konstitutiven und laufenden Kontrakte einer Unternehmung, die (informations-)technische Integration durch das KIS und die organisationale Integration durch die eingesetzten Koordinationsmechanismen.

Informationstechnische Integration wird mit dem Stratum Kommunikations- und Informationssystem (KIS) geschaffen. Sie meint das Abstimmen und Verbinden technischer Einheiten innerhalb und zwischen Unternehmungen. Ermöglicht wird das Abstimmen verschiedener Unternehmensteile, indem diese gemeinsam auf Daten zugreifen können (Shared data accessability), das Verbinden, indem Informationen in völlig neuer Form ausgetauscht werden können (Interconnectivity). ${ }^{1}$ Verbessertes Abstimmen und Verbinden technischer Einheiten ${ }^{2}$ sind Merkmale von KIS, die daher im Mittelpunkt der informationstechnischen Integration stehen. ${ }^{3}$ Dabei sind KIS vom Begriff der Informationstechnologie zu unterscheiden (vgl. Abb. A.4).4

1 Benjamin und Scott Morton unterscheiden in diesem Zusammenhang die Integration verschiedener formen von Transaktionsdaten und die Integration verschiedener Formen von Repräsentationen von Daten. Vgl. Benjamin, R.I., Scott Morton, M.S., Information Technology, Integration, and Organizational Change, Working Paper No. 138, Massachusetts Institute of Technology, Center for Information Systems Research, Cambridge, Massachusetts, 1986, S. 1.

2 Und indirekt Abstimmen und Verbinden der darauf aufbauenden nicht technischen Einheiten.

3 Vgl. Rockart, J.F., Short, J.E., IT in the 1990's, Managing Organizational Interdependence, in: Sloan Management Review, Vol. 30, Winter 1989, S. 30; Benjamin, R.I., Scott Morton, M.S., Information Technology, a.a.O., S. 12 ff; McKenney, J.L., McFarlan, F.W., The Information Archipelago - Maps and Bridges, in: Harvard Business Review, Vol. 60, No. 5, 1982, S. 110 ff; Costello, B., Systems Integration, in: Computing for Business, Vol. 10, Iss. 6, 1985, S. 63-65; Gitomer, J., Seamless systems Integration, in: Journal of Information Systems Management, Vol. 6, Iss. 3, 1989, S. 77 - 80; Day, M., The Art of Systems Integration, in PEM: Plant Engineering \& Maintenance (Canada), Vol. 13, Iss. 2, 1990, S. 40 - 44.

4 Es liegen verschiedene Taxonomien der Informationstechnologie vor, und zwar aus verschiedenen Blickwinkeln, in verschiedenen Kontexten und verschiedener Begrifflichkeit. Vgl. z.B. Szyperski, N., Winand, U., Informationsmanagement und informationstechnische Perspektiven, in: Organisation, Festschrift für Knut Bleicher, (Hrsg.), Seidel, E., Wagner, D., Wiesbaden, 1989, S. 137; Szyperski, N., Informationssysteme, computergestützte. In: Handwörterbuch der Organisation, (Hrsg.), Grochla, E., 2. Aufl., Stuttgart, 1980, S. 922; Kroeber, D.W., Watson, H.J., Computer Based Information Systems, 2nd ed., New York, 1987, S. 4 ff. In der deutschen Literatur werden die Begriffe definitorisch noch feiner untergliedert und daraus (Fortsetzung der Fußnote auf der nächsten Seite) 


\begin{tabular}{|c|c|}
\hline Informutionstechnologien & $\begin{array}{l}\text { Kommunlitions- und } \\
\text { Informutlonesyotom (KBS) }\end{array}$ \\
\hline $\begin{array}{l}\text { Hardware } \\
\text { Mainframe Computer } \\
\text { Minicomputer } \\
\text { Workstations } \\
\text { Personal Computer } \\
\text { Telekommunikationsnetzwerke } \\
\text { Softwer }\end{array}$ & $\begin{array}{l}\text { Transaktions- und Abwicklungseysteme } \\
\text { Managementinformationseysteme } \\
\text { Entschoidungsunterstutzungseysteme } \\
\text { Executhe Support Systems } \\
\text { Gruppenentscheidungsunterst.systeme } \\
\text { Büroautomatisierungseysteme } \\
\text { Compurterconferencing }\end{array}$ \\
\hline
\end{tabular}

Abb. A.4: Beispiele für Informationstechnologien und KIS

Als Informationstechnologie gilt die tatsächliche physische Technologie, einschließlich Software; als Kommunikations- und Informationssystem (KIS) die informationstechnisch basierten Infrastrukturen, die zur Erfüllung der betrieblichen Informationsfunktion dienen. ${ }^{1}$ In dieser Arbeit werden KIS betrachtet.

Der Grad der informationstechnischen Integration kann als Grad der Nutzung gemeinsamer Daten operationalisiert werden, d.h. als das Ausmaß, in dem Daten und Anwendungen redundanzfrei verwaltet werden. ${ }^{2}$ Dies manifestiert sich in mehrfacher Weise. In einem integrierten Kommunikations- und Informationssystem treten Daten einer gegebenen Art an einer einzigen Stelle in das System ein, werden redundanzarm konsoli-

Managementebenen abgeleitet. In der amerikanischen Literatur ist die Begrifflichkeit inkonsistent, allerdings werden Inhalte klarer abgegrenzt.

1 In einem umfassenden Sinn könnte die ganze Unternehmung als Informationssystem aufgefaßt werden. Der hier gewählte Begriff des Informationssystems bildet insoweit ein Subsystem des umfassenderen Systems Unternehmung. Zur Abgrenzung des Informationssystems der Unternehmung vgl. Meffert, H., Informationssysteme, Grundbegriffe der EDV und Systemanalyse, Tübingen, Düsseldorf, 1975, S. 26 f.

2 Vgl. Malone, Th.W., Yates, J., Benjamin, R.I., Electronic Markets and Electronic Hierarchies, in: Communications of the ACM, Vol. 30, June 1987, S. 495 f; Emery, J.C., Management Information Systems, a.a.O., 1987, S. 252 ff; Emery, J.C., Integrated Information Systems and their Effects on Organizational Structure, in: Information Systems, (Hrsg.), Grochla, E., Szyperski, N., a.a.O., S. 100; Scheer, A.-W., EDVorientierte Betriebswirtschaftslehre, 4. Aufl., Berlin u.a.O., 1990, S. 28 ff. Einen Zusammenhang von (technischer) Integration und verschiedenen Arten technischer Kompatibilität stellt Hübner her. Vgl. Hübner, H., Informationsmanagement, a.a.O., S. 109 ff. 
diert und an allen relevanten Stellen verfügbar. Darüber hinaus sind auch die Anwendungen und Programme konsolidiert. Insgesamt liegt eine hohe Konsistenz und eine niedrige Duplizierung von Aktivitäten vor. ${ }^{1}$ Scheer weist in diesem Zusammenhang auf zwei Teilprobleme der informationstechnischen Integration hin, und zwar die Integration von Prozeßketten einerseits und die Integration der Datenbasis andererseits. Dies entspricht der in dieser Arbeit vorgenommenen Unterscheidung der Prinzipien horizontaler Verflechtung und vertikaler Verknüpfung, bezogen auf das Stratum Kommunikations- und Informationssystem.

Organisationale Integration wird mit dem Stratum Koordinationsmechanismen geschaffen, ${ }^{2}$ wie zum Beispiel Anweisungen, Zielvorgaben, Verrechnungspreisregeln, Bildung von Teams. ${ }^{3}$ Koordinationsmechanismen sind das in der Literatur am umfassendsten ausgearbeitete Instrument zur Integration von Geschäftsaktivitäten. So unterscheiden Galbraith und Kazanjian als Integrationsinstrumente Hierarchie, Regeln, Zielvorgaben, Direktkontakt, Verbindungsstellen, gemeinsame Teams und eigens eingerichtete Integrationsabteilungen. ${ }^{4}$ Kieser und Kubicek unterscheiden persönliche Weisungen, Selbstabstimmung, Programme, Pläne und nichtstrukturelle Koordinationsinstrumente. 5 Meffert ordnet Koordinationsmechanismen bzw. die zugehörige Meta-Ebene der Konzepte über Koordi-

1 Emery, J.C., Organizational Structure, a.a.O., S. 99 ff. Scheer weist in diesem Zusammenhang auf zwei Teilprobleme der informationstechnischen Integration hin, und zwar die Integration von Prozeßketten einerseits und die Integration der Datenbasis andererseits. Dies entspricht der in dieser Arbeit vorgenommenen Unterscheidung der Prinzipien horizontaler Verflechtung und vertikaler Verknüpfung, bezogen auf das Stratum Kommunikation- und Informationssystem. Vgl. Scheer, A.-W., EDV-orientierte Betriebswirtschaftslehre, a.a.O., S. 46 f.

2 Vgl. Khandwalla, P.N., The design of organizations, New York, S. 157.

3 Vgl. Galbraith, J.R., Complex Organizations, a.a.O., S. 7; Galbraith, J.R., Kazanjian, R.K., Strategy Implementation. Structure, Systems, and Process, 2. ed., St. Paul u.a.O., 1986, S. 72; Lawrence, P.R., Lorsch, J.W., Organization and Environment, Boston, Massachusetts, 1967, S. 12. Eine genauere Beschreibung der einzelnen Koordinationsmechanismen erfolgt in Abschnitt B.2.3.1.2, hier seien nur Beispiele zur Illustration angegeben.

4 Vgl. Galbraith, J.R., Kazanjian, R.K., Strategy Implementation, a.a.O., S. 72; vgl. auch Galbraith, J.R., Complex Organizations, a.a.O., S. 7.

5 Kieser, A., Kubicek, H., Organizational Concept, a.a.O., S. 104 ff. 
nationsmechanismen in planungstechnokratische, strukturorientierte und kulturorientierte Ansätze. ${ }^{1}$ Aus der Perspektive dieser Arbeit ist auf den derart in der Literatur vorliegenden organisationalen Integrationsbegriff zurückzugreifen, jedoch das Spektrum der zu betrachtenden Integrationsinstrumente auf die KIS und die Kontrakte auszudehnen.

Organisationale Integration wird, Lawrence und Lorsch folgend, bezeichnet als die Qualität des Zusammenwirkens zwischen Unternehmensteilen, deren jeweilige Aufgabenumwelt ein solches Zusammenwirken erfordert. ${ }^{2}$ Der Grad der organisationalen Integration kann anhand von Kosiols Trichonomie kommunikativer Arbeitsbeziehungen operationalisiert werden: Information, Veranlassung und Willensbildung. ${ }^{3}$ Als organisational integriert werden Unternehmensteile aufgefaßt, die über eine gemeinsame Willensbildung gemeinsam Entscheidungen treffen. ${ }^{4}$ Dementsprechend sind Unternehmensteile organisational semi-integriert (schwach integriert), wenn sie durch Veranlassung (durch Informationsaustausch) auf die gegenseitige Entscheidungsfindung einwirken. Organisational nicht integriert sind Unternehmensteile, die unabhängig voneinander Entschei-

1 Vgl. Meffert, H., Klassische Funktionenlehre, a.a.O., S. 386; Benkenstein, M., F\&E und Marketing Bd. 20 der Schriftenreihe Unternehmensführung und Marketing, (Hrsg.), Meffert, H., Steffenhagen, H., Freter, H., Wiesbaden 1987 S. 122 ff.

2 Lawrence, P.R., Lorsch, J.W., Organization and Environment, a.a.O., S. 11. Diese Definition ist eng verwandt mit dem Begriff der Koordination. Während Integration die Verbindung von Elementen zu einem Ganzen bedeutet, kann Koordination verstanden werden als die Abstimmung von Einzelaktivitäten im Hinblick auf ein konkretes Ziel. Damit ist Integration das umfassendere Konstrukt, Koordination ist gleichwohl wesentlich zum Erreichen eines integrierten Zustandes. Vgl. Frese, E., Koordination, a.a.O., Sp. 2263; Adam, D., Koordinationsprobleme bei dezentralen Entscheidungen, in: Zeitschrift für Betriebswirtschaft, 39. Jg., Oktober 1969, S. 618.

3 Kosiol, E., Organisation der Unternehmung, 2. Aufl., Wiesbaden, 1976, S. 179.

4 Vgl. March, J.G., Simon, H.A., Organizations, a.a.O., S. 122. March und Simon zeigen darüber hinaus, daB die Notwendigkeit gemeinsamer Entscheidungsfindung von der Stärke vorliegender Interdependenzen bedingt ist. Dabei führen sie zwei Faktoren als bedeutsam an: die Nutzung gemeinsamer Ressourcen und die (zeitliche) Interdependenz nacheinandergelagerter Aktivitäten. Dies ist konsistent mit den Integrationsprinzipien horizontaler Verflechtung (gleichartige Ressourcen) und vertikaler Verknüpfung (nacheinandergelagerte Geschäftsaktivitäten). 
dungen treffen, d.h. zwischen denen keinerlei Koordinationsmechanismen bestehen.

Der Begriff der organisationalen Integration ist vom Begriff der Koordination abzugrenzen. Koordination ist nicht gleich Integration, vielmehr umfaßt Koordination die Abstimmung von Entscheidungen und Handlungen im Rahmen bestehender Strukturen," während Integration die zielorientierte Ausrichtung von Strukturen zu einer Ganzheit ist. ${ }^{2}$ Mithin wird im Rahmen der Koordination spezifiziert, was durch die Integration strukturiert wurde. ${ }^{3}$ Folglich ist (organisationale) Integration die Schaffung integrativer Verbindungen durch die Auswahl von Koordinationsmechanismen. Beispiele dafür sind die Einführung eines Verrechnungspreissystems oder Zielvorgabenmechanismus, etwa Management by Objectives $^{4}(\mathrm{MbO})$. Demgegenüber ist Koordination die Implementierung im Rahmen eines gegebenen Koordinationsmechanismus. Beispiel dafür sind die Wahl eines Verrechnungspreises im Rahmen des Verrechnungspreissystems oder im Rahmen des Management by Objectives (MbO) die Vereinbarung eines konkreten Zielinhalts oder -ausmaßes. ${ }^{5}$

Kontraktuelle Integration wird mit dem Stratum Kontrakte geschaffen. Der Begriff der kontraktuellen Integration basiert auf der Frage von Coase, ob es vorteilhafter ist, Geschäftsaktivitäten innerhalb der Grenzen der Un-

1 Vgl. Kieser, A., Kubicek, H., Organisation, a.a.O., S. 329 ff; Kosiol, E., Organisation, a.a.O., S. 172; Mellerowicz, K., Unternehmenspolitik, Bd. 1, Freiburg i. Br., 1963, S. 243.

2 Vgl. Kosiol, E., Aufbauorganisation, in: Handwörterbuch der Organisation, (Hrsg.), Grochla, E., Stuttgart, 1969, S. 55; Lawrence, P.R., Lorsch, J.W., Organization and Environment, a.a.O., S. 11; Heinen, E., Industriebetriebslehre, a.a.O., S. 25.

3 Vgl. Kosiol, E., Organisation, a.a.O., S. 172 f.

4 Vgl. Wöhe, G., Einführung in die Allgemeine Betriebswirtschaftslehre, 14. Aufl., München, 1981, S. 124.

5 Auf eine ausführliche etymologische oder dogmengeschichtliche Diskussion der Begriffe der Integration und Koordination wird verzichtet, diese liegt in der Literatur mehrfach vor. Gute Übersichten finden sich bei Vieweg, H.-G., Koordination: die Entwicklung eines Modells und seine Anwendung in einer empirischen Untersuchung, München, 1987, S. $2 \mathrm{ff}$, bes. S. 12 ff und S. 31 ff; Hübner, H., Integration und Informationstechnologie im Unternehmen, München, 1979, S. 13 ff, bes. S. 30 - 32 . 
ternehmung oder über den Markt abzuwickeln. ${ }^{1}$ Ein Unternehmensteil ist kontraktuell in eine Unternehmung integriert, wenn er innerhalb der Grenze der Unternehmung angesiedelt ist, beispielsweise durch Arbeitsverträge. Demgegenüber sind kontraktuell nicht integriert Teile anderer Unternehmungen, beispielsweise Lieferanten, mit denen einfache Kaufverträge geschlossen werden. ${ }^{2}$ Das Stratum Kontrakte umfaßt die konstitutiven und laufenden Kontrakte, in denen die Rechte und Pflichten der Unternehmensteile festgelegt werden, die an den betrachteten Geschäftsaktivitäten beteiligt sind. In diesen Kontrakten sind insbesondere die von Zukunftskontingenzen abhängigen Zahlungen spezifiziert.

Kontraktuelle Integration ist keine 0-1 Variable, d.h. manche Unternehmensteile sind partiell kontraktuell integriert bzw. "quasi-integriert". Solche Zwischenformen der Quasi-Integration sind beispielsweise Allianzen, Franchise-Vereinbarungen, Joint Ventures, 'freie' Mitarbeiter oder Beteiligungen. ${ }^{3}$ Der Grad kontraktueller Integration kann als Ausmaß der gemeinsamen Zielfunktion zweier Unternehmensteile operationalisiert werden, und zwar in Anlehnung an das Konzept der vertikalen Integra-

1 Coase, R., On the Nature of the Firm, in: Economica, Vol. 4, 1937, S. 333. Dieser Artikel Coase's begründete den Transaktionskostenansatz, dessen Grundfrage es ist, warum Unternehmungen existieren, d.h. warum nicht sämtliche Transaktionen (Elementarkombinationen und Tauschakte) über Märkte abgewickelt werden. Grundaussage des Transaktionskostenansatzes ist es, daß über den Markt abgewickelte Transaktionen Kosten verursachen, sogenannte Transaktionskosten, z.B. für die Suche von Tauschpartnern, Vertragsaushandlung, Steuern, Vertragscontrolling usw. Aufgrund dieser Transaktionskosten werden viele Transaktionen nicht über den Markt, sondern innerhalb von Unternehmungen abgewickelt. Einen Überblick über den Transaktionskostenansatz geben Schumann, J., Grundzüge der miknökonomischen Theorie, 3. Auflage, Berlin, Heidelberg, New York, 1980; Williamson, O.E., Comparative Economic Organization - Vergleichende ökonomische Organisationstheorie: Die Analyse diskreter Strukturalternativen, in: Betriebswirtschaftslehre und ökonomische Theorie, (Hrsg.), Ordelheide, D., Rudolph, B., Büsselmann, E., Stuttgart, 1991.

2 Zum Begriff der Firmengrenze vgl. MacMillan, K., Farmer, D., Boundaries of the Firm, a.a.O.; Williamson, O.E., Economic Institutions, a.a.O., S. 96 ff.

3 Vgl. MacNeil, I., The New Social Contract. An Inquiry into Modem Contractual Relationship, New Haven, 1980, S. 71 f. Die kontraktuelle Integration wird häufig vor allem auf die vertikale Integration bezogen. Vgl. dazu Blois, K.J., Vertical QuasiIntegration, in: The Journal of Industrial Economics, Vol. 20, 1972, S. 253 - 272; Benkenstein, M., Henke, N., Vertikale Integration - Eine transaktionskostentheoretische Interpretation, Arbeitspapier Nr. 39 des Instituts für (Fortsetzung der Fußnote auf der nächsten Seite) 
tion (Eigenherstellung oder Fremdbezug, z.B. am Spotmarkt). ${ }^{1}$ Zwei Unternehmensteile weisen einen hohen kontraktuellen Integrationsgrad auf, wenn sie gemeinsam ihr (bzw. ein übergeordnetes) Unternehmensergebnis zu optimieren haben. Ein niedriger kontraktueller Integrationsgrad liegt bei jeweils isolierter Optimierung unterschiedlicher Zielfunktionen vor. ${ }^{2}$ Kontrakte über den Fremdbezug an einem Spotmarkt werden mit "Externen" geschlossen. Um Abweichungen von geschlossenen Vereinbarungen zu vermeiden, werden dabei Produktionsqualitäten, -mengen, -preise und -termine bestimmt. Demgegenüber werden bei Eigenherstellung langfristige, offene Verträge mit den Eigentümern der eingehenden Produktionsfaktoren geschlossenen, die erst nach Vertragsschluß genauer spezifiziert werden. Damit werden diese Produktionsfaktoren langfristig in das System Unternehmung integriert, d.h. kontraktuelle integrative Verbindungen zwischen den Eigentümern der Produktionsfaktoren hergestellt. ${ }^{3}$

Drei unterschiedliche Integrationsbegriffe wurden aus der betriebswirtschaftlichen, informationstechnischen, organisationstheoretischen und institutionenökonomischen Literatur abgeleitet. Während bisher vorliegende Taxonomien von Integrationsinstrumenten jeweils im wesentlichen auf einem der drei unterschiedenen Integrationsbegriffe fußen, 4 liegt mit der Zuordnung je eines Integrationsinstruments (KIS, Koordinationsmechanismen und Kontrakte) auf die Integrationsbegriffe eine Systematik von Integrationsinstrumenten vor, die das Integrationsproblem wesentlich breiter faßt als die bisher vorliegende Literatur.

Marketing, Münster, 1990, S. 1 ff.

1 Vgl. Benkenstein, M., Henke, N., Vertikale Integration , a.a.O., S. 3 - 9.

2 Vgl. Abschnitt B.2.3.1.3 Kontrakte; beachte Subzielverfolgung von Agenten, Pratt, J.W., Zeckhauser, R.J. (Hrsg.), Principals and Agents: The Structure of Business, Boston, Massachusetts, 1985; Williamson, O.E., The Economics of Discretionary Behavior: Managerial Objectives in the Theory of the Firm, Englewood Cliffs, New Jersey, 1964 .

3 Vgl. Williamson, O.E., Economic Institution, a.a.O., S. 85 ff, bes. S. 90 ff.

4 Beispiele sind Galbraith, der einige Koordinationsmechanismen und das ITS unterscheidet. Vgl. Galbraith, J.R., Complex Organizations, a.a.O., S. 15; Galbraith, J.R., Kazanjian, R.K., Strategy Implementation, a.a.O., S. 72. Weiterhin Vgl. Meffert, H., Klassische Funktionenlehre, a.a.O., S. 386. 


\subsection{Wettbewerbsstrategische Bedeutung von Kommunikations- und Informationssystemen (KIS)}

In der Literatur wird die große wettbewerbsstrategische ${ }^{1}$ Bedeutung von KIS überwiegend anhand der wettbewerbsstrategischen Ansätze von Porter (fünf Determinanten der Branchenprofitabilität, Wertkette) aufgezeigt. ${ }^{2}$ Der strategische Einsatz von KIS zur Integration der Unternehmung kann den Wettbewerb in Bezug auf jede der fünf Determinanten der Struktur und Profitabilität einer Branche verändern.

1 Auf eine allgemeine Erläuterung des Strategiebegriffs wird hier verzichtet, da dieser in der Literatur extensiv dokumentiert ist. Einen Überblick geben Meffert, $H$., Wertkette, a.a.O., bes. S. 3 ff; Meffert, H., Marketing, Grundlagen der Absatzpolitik, Wiesbaden, 1986, S. 54 ff; Gluck, F.W., Kaufmann, S.P., Walleck, A.S., Strategic Management for Competitive Advantage, in: McKinsey Quarterly, 1980; Hinterhuber, H.H., Strategische Unternehmensführung, Berlin, New York, 1980. Zur Unterscheidung der Konzepte der Strategie, der strategischen Unternehmensführung sowie der strategischen Planung vgl. Scholz, Chr., Strategisches Management, Berlin, New York, 1987, bes. S. 4 ff; Hahn, D., Strategische Unternehmensplanung. Ein konzentrierter Überblick, in: WISU, 10. Jg., Nr. 6, 1981, S. 275 - 279; Meffert, H., Wertkette, a.a.O., S. 257 ff. Jüngere Darstellungen, einschließlich der etymologischen Wurzeln und definitorischer Abgrenzungen eines Begriffs der Strategie bzw. Wettbewerbsstrategie zu verwandten Begriffen, finden sich bei Dannenberg, J., Mikrocomputergestützte Instrumente der strategischen Unternehmensplanung, Wiesbaden, 1990, S. 16 ff; Overlack, J., Wettbewerbsvorteile durch Informationstechnologie, Frankfurt a. M. u.a.O., 1988 S. 31 ff.

2 Vgl. Porter, M.E., Competitive Strategy, New York, New York, 1980, S. 4; Parsons, G.L., Information Technology: A New Competitive Weapon, in: Sloan Management Review, Vol. 25, Fall 1983, S. 3 - 13; Porter, M.E., Competitive Advantage, a.a.O., 1985, S. 37; Porter, M.E., Millar, V.E., Information, a.a.O. Andere Ansätze sind Wiseman, Ch., Strategy and Computers, Homewood, Illinois, 1985, S. 9 und 42 ff; Porter, M.E., Millar, V.E., Information, a.a.O., S. 27; Mertens, P., Plattfaut, E., Informationstechnik als strategische Waffe, in: Information Management, Nr. 2, 1986, S. 6; Krüger, W., Pfeiffer, $P$., Eine konzeptionelle und empirische Analyse der Informationsstrategien und der Aufgaben des Informationsmanagements, in: Zeitschrift für betriebswirtschaftliche Forschung, 43. Jg., 1991, S. 22. Eine Übersicht anderer Ansätze stellt Overlack, J., Wettbewerbsvorteile, a.a.O., S. 102 ff zusammen. In der älteren Literatur wird weniger die wettbewerbsstrategische Bedeutung des Einsatzes von KIS diskutiert, sondern entweder der Einsatz von KIS zur strategischen. Unternehmensplanung oder die langfristige Planung des Informationssystems. Einen Überblick gibt Overlack, J., Wettbewerbsvorteile, a.a.O., S. 32; vgl. auch Mertens, P., Plattfaut, E., Ansätze zur DV-Unterstützung der strategischen Unternehmensplanung, in: Die Betriebswirtschaft, Vol. 43, (Fortsetzung der Fußnote auf der nächsten Seite) 
KIS können die Nachfragemacht der Käufer senken, indem die Geschäftsaktivitäten über Kommunikationssysteme abgewickelt und die Umschaltkosten der Käufer von einem auf einen anderen Hersteller erhöht werden. ${ }^{1}$ Andererseits können KIS die Marktübersicht der Käufer erleichtern und so deren Verhandlungsposition verbessern. ${ }^{2}$ Zulieferer können Anbietermacht einbüßen, weil sie die Abwicklung der Transaktionen mit Produzenten automatisieren, wodurch die Qualität besser kontrollierbar und die Kosten besser erkennbar werden. Der Einsatz von KIS hat auch zur vielfältigen Substitution von Produkten und Leistungen geführt. Beispielsweise wird ein großer Teil von Eilzustellungen nicht mehr von $\mathrm{Zu}$ stelldiensten, sondern über Telekommunikation ausgeführt, insbesondere durch Facsimile-Übertragung. CAD und CAM-Technologien erlauben eine schnellere und einfachere Entwicklung von Substitutionsprodukten in vielen Märkten. Der Einsatz von KIS kann Marktschranken für neue Konkurrenten aufbauen. Beispielsweise binden Informationsnetzwerke Zulieferer oder Distributionskanäle fest an den Netzwerkbetreiber, wie z.B. im britischen Non-food-Großhandel oder im US-amerikanischen Pharmagroßhandel. ${ }^{3}$ Schließlich beeinflussen KIS auch stark die Wettbewerbsintensität. In nahezu allen Branchen, in denen KIS einen bedeutenden Anteil der Geschäftsaktivitäten unterstützen, z.B. bei Anbietern von Flugreisen, Finanz- und Bankdienstleistungen sowie Informationen oder im Einzelhandel, hat sich die Wettbewerbsintensität verändert, im allgemeinen erhöht. 4

Angesichts der großen Bedeutung von KIS für die fünf Determinanten des Wettbewerbs erhebt sich die Frage nach den konkreten Auswirkungen

1985, S. 19 - 30.

1 Vgl. Parsons, G.L., Information Technology, a.a.O., S. 7.

2 Vgl. Porter, M.E., Millar, V.E., Information, a.a.O., S. 155.

3 Vgl. Vitale, M.R., Earl, R., British Home Supply (BhS), Boston, Massachusetts, 1988; Clemons, E.K., Row, M., McKesson Drug Company: A Case Study of Economost - A Strategic Information System, in: Journal of Management Information Systems, Vol. 5, Summer 1988, S. 36. 
auf einzelne Geschäftsaktivitäten. Diese werden häufig anhand der Porterschen Wertkette in neun Kategorien aufgespalten mit dem Ziel, deren jeweiligen Beitrag zum Kosten- oder Differenzierungsvorteil zu bestimmen. ${ }^{1}$ In Abbildung A.5 sind den Kategorien von Geschäftsaktivitäten jeweils beispielhaft KIS zugeordnet. ${ }^{2}$

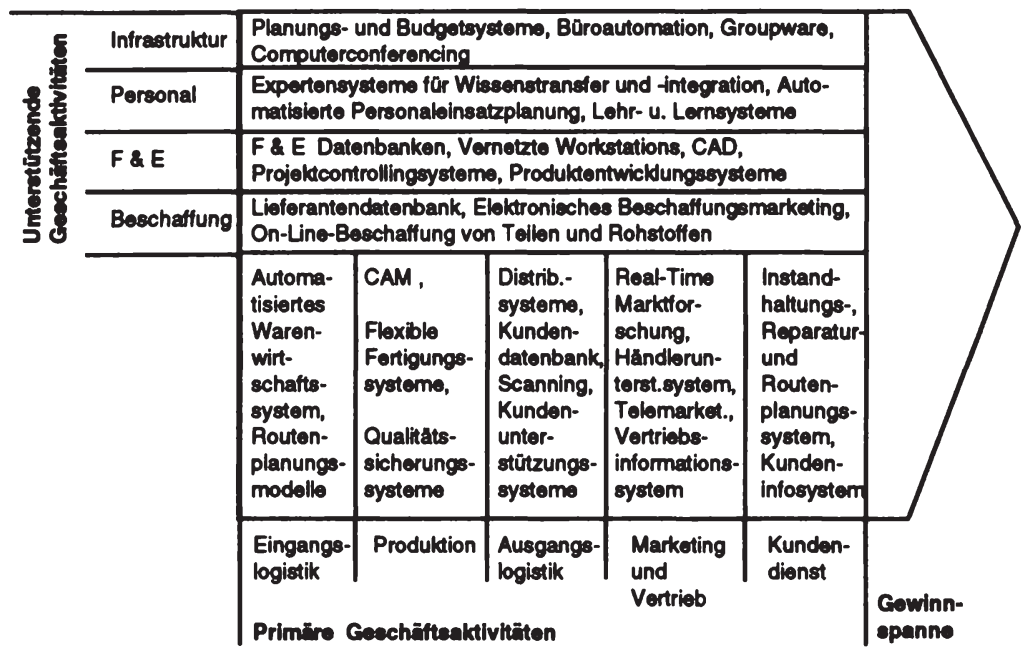

Abb. A.5: Beispiele für KIS in der Wertkette

Quelle: Porter, M.E., Millar, V.E., Information, a.a.O., S. 153, ursprüngl.: Parsons, G.L., Information Technology, a.a.O., S. 7.

Beispielsweise mögen die Kosten der Ausgangslogistik eines Nahrungsmittelherstellers wesentlich vom Einsatz eines Ausgangslogistiksystems abhängen, weil die Verknüpfung mit dem Distributionskanal enger wird, regionale Skaleneffekte ausgebaut werden und gegebene Servicegrade bzw. Lieferzeiten durch zunehmende Vertriebseffizienz kostengünstiger erbracht werden können.

4 Vgl. Porter, M.E., Millar, V.E., Information, a.a.O., S. 153 ff.

1 Vgl. Porter, M.E., Competitive Advantage, a.a.O., S. 45; Meffert, H., Wertkette, a.a.O., S. 263.

2 Vgl. Parsons, G.L., Strategic Information Technology, Boston, Massachusetts, 1983, S. 12; (Fortsetzung der Fußnote auf der nächsten Seite) 
Durch den Einsatz von KIS wurde die Integrationsstrategie in vielen Unternehmen ein entscheidender Faktor für den Unternehmenserfolg. Der italienische Modehersteller Benetton integriert Beschaffung, Produktion und Distribution durch ein weltweites Informationssystem. Täglich werden Abverkaufszahlen weltweit nach einzelnen Produkten erfaßt, so daß Marketing- und Produktionsentscheidungen nach den jeweils neuesten Informationen getroffen werden können. Damit nimmt die Qualität der Entscheidungen zu, beispielsweise in der Produktion (Farbe, Menge) oder Preispolitik.1 US-amerikanische Vermögensverwaltungsfirmen integrieren ihre Geschäftsaktivitäten Research, Anlagestrategie, Kundenkontakt, Handel und Abwicklung durch KIS. Dies bedeutet eine fundamentale Restrukturierung der Unternehmung mit erheblichen Kostensenkungen. ${ }^{2}$ Amerikanische Schnellrestaurant-Ketten integrieren ihre Aktivitäten im Distributionskanal mit Teilhaber- oder Franchisingverträgen und Informationssystemen. Indem sie global vergleichbare Qualitätsstandards bei kürzesten Zubereitungszeiten gewährleisten, erreichen sie schwer angreifbare Wettbewerbspositionen. Japanische Automobilunternehmen integrieren die einzelnen Geschäftsaktivitäten in der Beschaffung, Produktion und Distribution durch enge ablauforganisatorische Koordinationsmechanismen und Informationssysteme. Sie reduzieren die Durchlaufzeiten auf Bruchteile der Ausgangsbasis bei sinkenden Kosten und steigender Qualität. ${ }^{3}$

Porter, M.E., Millar, V.E., Information, a.a.O., S. 153.

1 Vgl. Vitale, M.R., Benetton S.p.A.: Industrial Fashion (A), Boston, Massachusetts, 1988; Sauter, E., Making the Right Connections, in: World, Vol. 21, No. 3, July - September 1987, S. 36; Zottola, L. The United Systems of Benetton, in: Computerworld, Vol. 24, No. 14, 1990, S. 70.

2 Benjamin, R.I., De Long, D.W., Scott Morton, M.S., The Realities of Electronic Data Interchange: How Much Competitive Advantage?, Working Paper No. 166, Massachusetts Institute of Technology, Center for Information Systems Research, Cambridge, Massachusetts, 1986, S. 6 ff; Schmerken, I., Expert Systems Churns Many Happy Returns, in: Wall Street Computer Review, Vol. 5, No. 3, December 1987, S. 17.

3 Shingo, Sh., A revolution in manufacturing: the SMED system. Stamford, Connecticut, 1985, S. 121 ff; Blackburn, J.D., Time-Based Competition, a.a.O., S. 44 ff. 


\subsection{Wettbewerbsstrategische Bedeutung der Veränderung von Organisation und Unternehmensgrenze durch KIS}

Neue Integrationsmöglichkeiten ergeben sich nicht nur aus dem Einsatz von KIS selbst, sondern zwei in der Literatur beschriebenen Wirkungen von KIS auf die Unternehmung: die Dezentralisierung von Organisationen und die Veränderung der Grenzen der Unternehmung. Diese verlaufen parallel zu dem immer umfassenderen Einsatz von KIS und scheinen sich wechselseitig zu beeinflussen, so daß man von einer Substitutionalität von Integrationsmechanismen sprechen kann.

Die Dezentralisierung von Organisationen wurde bereits in der früheren Literatur aus der Möglichkeit zur besseren Informationsverarbeitung ${ }^{1}$ gefolgert. Daraus wurde die Prognose abgeleitet, daß Organisationen in der Zukunft flacher sein würden. ${ }^{2}$ Neben organisatorischer Dezentralisierung verändern sich die Grenzen der Unternehmung. ${ }^{3}$ Im wesentlichen auf Basis transaktionskostenökonomischer Überlegungen 4 wird in der

1 Vgl. z.B. Whisler, Th.L., Man, Organization, and Computer - A Contingency Analysis, in: Grochla, E., Szyperski, N., Information Systems, a.a.O., S. 246 ff und S. 255 f. Dabei wurde vor allem an die Reduzierung der kognitiven Grenzen des Menschen gedacht. Vgl. Simon, H.A., Administrative Behavior, New York, New York, 1976, S. XXXI; March, J.G., Simon, H.A., Organizations, New York, New York, 1958, S. 138.

2 Vgl. Drucker, P. F., The Coming of the New Organization, in: Harvard Business Review, Vol. 66, 1988, S. 45 - 53; Applegate, L.M., Cash, J.I., Mills, D.Q., Information Technology and Tomorrow's Manager, in: Harvard Business Review, Vol. 66, November - December, 1988, S. 128 ff; Leavitt, Th., Whisler, Th.L., Management, a.a.O., S. 41. Eine aktuelle Reflektion beider Autoren findet sich bei Applegate, L.M., Cash, J.I., Mills, D.Q., Information Technology, a.a.O., S. 133. Vgl. außerdem Picot, A., Kommunikationstechnik und Dezentralisierung, in: Information und Wirtschaftlichkeit, (Hrsg.), Ballwieser, W., Berger, K. H., Wiesbaden 1985; Zuboff, Sh., Smart Machine, a . a. O., Kapitel 9; Peters, T., The Destruction of Hierarchy, in: Industry Week, August 15, 1988, S. 33 - 35; Miles, R. E., Snow, Ch. C., Network Organization - New Concepts for New Forms, in: McKinsey Quarterly, Autumn, 1986, S. 53 - 66; Jarillo, J. C., Ricard, J. E., Sustaining Networks, in: Interfaces, Vol. 17, No. 5, 1987, S. 82 - 91; Thorelli, H. B., Networks, Between Markets and Hierarchies, in: Strategic Management Journal, Vol. 7, No. 1, 1986, S. 37 - 51.

3 MacMillan, K., Farmer, D., Redefining the Boundaries of the Firm, in: Journal of Industrial Economics, Vol. 27, No. 3 , 1979, S. 277 - 285.

4 Vgl. Williamson, O.E., Transaction Cost Economics: The Governance of Contractual Relations, in: Journal of Law and Economics, Vol. 22, 1979, S. 3 - 61; Williamson, O.E., (Fortsetzung der Fußnote auf der nächsten Seite) 
Literatur argumentiert, daß sich gleichzeitig mit dem Einsatz von KIS die optimalen vertraglichen Beziehungen innerhalb und zwischen Unternehmen verändern. ${ }^{1}$

Scheinbar bedingen sich Veränderungen der KIS, von Organisationsstrukturen bzw. -abläufen und der Grenzen der Unternehmung gegenseitig. Diese Bedingtheit kann als Substitutionsprozeß verschiedener, alternativ einsetzbarer Integrationsmethoden interpretiert werden. Neue KIS substituieren, (zumindest verändern) organisatorische und firmenkonstituierende Kontrakte. Ein Indiz hierfür findet sich in der Entwicklung der relativen Kosten von KIS. Substitutionsprozesse sind häufig von der Entwicklung der relativen Kosten getrieben. Die Produktivität der Informationstechnologien ist in den letzten 10 Jahren gegenüber der Arbeitsproduktivität um das 25-fache gewachsen. Dies läßt die Existenz solcher Substitutionsprozesse vermuten. ${ }^{2}$ Aufgrund des geschilderten substitutiven $\mathrm{Zu}$ -

The Economic Institutions of Capitalism, New York, 1985; Benkenstein, M., Henke, N., Vertikale Integration - Eine transaktionskostentheoretische Interpretation, Arbeitspapier Nr. 39 des Instituts für Marketing, Münster, 1990.

1 So fordern MacMillan und Farmer eine Redefinition der Grenzen der Firma. MacMillan, K., Farmer, D., Boundaries of the Firm, a.a.O., S. 277. Johnston und Lawrence sehen anstelle einer als vertragliche Einheit integrierten Unternehmung lose Verbünde von Unternehmen, sogenannte "Value-Adding Partnerships", Johnston, H.R., Lawrence, P.R., Beyond Vertical Integration - The Rise of the Value-Adding Partnership, in: Harvard Business Review, Vol. 66, July - August 1988, S. 94 - 101; Konsynski und McFarlan ähnlich sogenannte "Information Partnerships". Konsynski, B.R., McFarlan, F.W., Information Partnerships Shared Data, Shared Scale, in: Harvard Business Review, September October 1990, S. 114 - 120; Malone, Yates und Benjamin die wachsende Bedeutung elektronischer Märkte anstelle von Geschäftsaktivitäten, die vormals innerhalb von Unternehmensgrenzen abgewickelt wurden. Malone, Th.W., Yates, J., Benjamin, R.I., Electronic Markets, a.a.O., S. 489; Malone, Th.W., Yates, J., Benjamin, R.I., The Logic of Electronic Markets, in: Harvard Business Review, Vol. 67, May - June 1989, S. 166 - 170.

2 Benjamin, R.I., Scott Morton, M.S., Information Technology, a.a.O., S. 2; Scott Morton, M.S., The Corporation of the 1990 s - Information Technology and Organizational Transformation, New York, Oxford, 1991, S. 9 - 11. Auf anderem Wege zeigt dies auch Venkatraman, N., Strategic Management and Information Technology: Evolutionary Linkages and a Research Framework, in: Strategy, Onganisation Design and Human Resource Management, (Hrsg.), Snow, C.C., Greenwich, Conneticut, London, England, 1989, S. 145; Die Signifikanz der informationstechnologischen Veränderungen wird in der Literatur an den verschiedensten Tendenzen und Kennziffern gezeigt. Vgl. Leontief, W., The Choice of Technology, in: Scientific American, Vol. 252, Jume 1985, S. 37 ff; Porter, M.E., Millar, V.E., Information, a.a.O., S. 152; Meyer, N.D., Boone, M.E., The Information Edge, New York, New York, 1987, S. 3 - 10; McKenney, J.L., McFarlan, F.W., Information Archipelago, a.a.O.S. 110 ff.; Einen guten Überblick gibt Zuboff, Sh., Smart Machine, a.a.O., S. 415 ff. 
sammenhangs geht die wettbewerbsstrategische Bedeutung der Integration von Geschäftsaktivitäten über die (hohe) Bedeutung des Einsatzes von KIS hinaus. Veränderungen von KIS, Organisationen und vertragliche Strukturen bedingen sich gegenseitig und sind bei der Betrachtung der Integration von Geschäftsaktivitäten als zusammenhängend anzusehen. Die Substitutionalität zwischen KIS, Organisationen und vertraglichen Strukturen wird dabei Grenzen haben, d.h. als periphere Substitution bzw. Randsubstitution gelten. 1

\subsection{Wettbewerbsstrategische Bedeutung des Zwangs- und Bindungs charakters von Integrationsstrategien}

Die wettbewerbsstrategische Bedeutung von Integration wird durch den "Zwangs- und Bindungscharakter" von Integrationsstrategien noch verstärkt, insbesondere bei hohen Investitionserfordernissen für neue KIS: Mit einer Umstellung auf eine neue Integrationsstrategie können Aufbau, Abläufe und/oder Grenzen der Unternehmung - wie skizziert - erheblich verändert werden. Aufgrund des Ausmaßes (und Aufwandes) solcher Veränderung ist das Unternehmen jedoch für einen längeren Zeitraum an eine Integrationsstrategie gebunden. Dies gilt beispielsweise für die frühen Nutzer von Informationssystemen aus informationsintensiven Branchen, z.B. Banken und Versicherungen, die über einen gewissen Zeitraum an rasch veraltende KIS (und die zugehörige Ablauforganisation und Vertragsstruktur) gebunden sind. Die Umstellung der Abwicklungsroutinen zwischen der Unternehmung, den Lieferanten, dem Handelskanal und den Kunden auf KIS erfordert die Ausrichtung der Geschäftsaktivitäten, Organisation und vertraglichen Beziehungen hierauf. Dies verursacht nicht nur einen erheblichen Umstellungs- und Lernaufwand, auch

1 Periphere oder Randsubstitutionalität von Produktionsfaktoren liegt vor, wenn ein Austausch zwischen Produktionsfaktoren nur in Grenzen möglich ist. Vgl. Gutenberg, E., Produktion, Berlin, Heidelberg, New York, 1983, S. 301 f; Heinen, E. , Kostenlehre, Wiesbaden, 1983, S. 199 f. 
würde jede weitere Veränderung erheblichen neuen Aufwand nach sich ziehen. Deswegen gehen die Beteiligten eine Bindung für einen größeren Zeitraum ein, über dessen Verlauf eine Amortisation der Ausgangsinvestition anzustreben ist.

Neben dem Bindungscharakter haben Integrationsstrategien häufig auch einen Zwangscharakter. Aufgrund der hohen strategischen Bedeutung von KIS erscheint jedes einzelne Unternehmen gezwungen, an "neuen Regeln" zu partizipieren und seinerseits Investitionen vorzunehmen, z.B. in eine neue Generation von KIS. ${ }^{1}$ Diese Feststellung entspricht einer Beobachtung Peters. Er berichtet über die erstaunliche Geschwindigkeit bei der Ausbreitung neuer KIS. Meist reiche es aufgrund ihrer erheblichen wettbewerbsstrategischen Bedeutung schon aus, daß ein Unternehmen KIS zur Integration von Geschäftsaktivitäten einsetzt, um eine rasche, branchenweite Imitation der Integrationsstrategie auszulösen. ${ }^{2}$ Die rasche Ausbreitung von KIS-gestützten Integrationsstrategien werde dadurch erleichtert, daß KIS-Anwendungen entweder allen Wettbewerbern zugänglich sind oder der in selbstentwickelten KIS ruhende technologische Wissensvorsprung rasch kopierbar ist. ${ }^{3}$ Deswegen kann sich der Zuschnitt ganzer Unternehmungen oder Branchen erheblich verändern. ${ }^{4}$ Hieraus resultiert jedoch ein Dilemma. Unternehmen können gezwungen sein, sich an die Integrationsstrategien der Wettbewerber anzupassen. Die dann erreichte Wettbewerbsposition kann aber wegen der eingegangenen Bindungen nicht beliebig verändert werden. Das heißt, Angriffe von Konkurrenten können die Ausschöpfung einer Wettbewerbsposition erschweren bzw. verhindern. Notwendig ist somit deren Absicherung über den

1 Vgl. z.B. Nagel, K., Bewertung strategischer Wettbewerbsvorteile durch Informationssysteme, in: Spremann, K., Zur, E., Informationstechnologie und strategische Führung, Wiesbaden, 1989, S. 50 ff.; Porter, M.E., Millar, V.E., Information, a.a.O., S. 149.

2 Vgl. Peters, T., Destruction of Hierarchy, a.a.O., S. 34 f.

3 Cecil, J., Goldstein, M., Sustaining competitive advantage from IT, in: McKinsey Quarterly, 1990, S. 75 f.

4 Dies deckt sich mit den Ergebnissen aus einer zweijährigen longitudinalen Feldstudie mit 16 Großunternehmen durch Rockart und Short, vgl. Rockart, J.F., Short, J.E., IT in the 1990's, a.a.O., S. 9 f. 
Zeitraum, für den sie fixiert wurde. Deswegen ist es nicht allein hinreichend, die Bedeutung von Integration für einen (aktuellen) Wettbewerbsvorteil zu betrachten. Darüber hinaus ist die Dauerhaftigkeit des Wettbewerbsvorteils im Zeitablauf zu analysieren. Das Problem der Dauerhaftigkeit von Wettbewerbsvorteilen mit Bindungs- und Zwangscharakter ist bei der Bestimmung von Ziel und Gang dieser Arbeit zu berücksichtigen.

\section{Ziel und Gang der Arbeit}

Trotz der fundamentalen theoretischen und praktischen Bedeutung der Integration von Geschäftsaktivitäten liegt kein umfassender Ansatz vor, der mehr als nur Teile der aufgeworfenen Fragen strukturiert. So verfügen Rockart und Short zwar über ein Raster von Forschungsfragen, welche Auswirkungen der Einsatz von KIS auf die Unternehmung hat, beklagen jedoch selbst die "absence of any powerful model wich can link these dimensions of organizational change to actual performance changes in the firm". ${ }^{1}$ Diese Arbeit soll dazu beitragen, dieses Defizit zu reduzieren.

Das Ziel dieser Arbeit ist es daher, die wettbewerbsstrategische Wirkung der Integration von Geschäftsaktivitäten zu analysieren, dies unter besonderer Berücksichtigung des Einsatzes von KIS. Es soll ein Bezugsrahmen der Integration von Geschäftsaktivitäten entwickelt werden, auf dessen Basis Integrationsstrategien konkretisiert und im Hinblick auf ihre wettbewerbsstrategische Wirkung analysiert werden können. Basis der Analyse ist ein geeignetes betriebswirtschaftliches Grundmodell, mit dem verschiedene Integrationsstrategien unterschieden, beschrieben und im Hinblick auf ihre wettbewerbsstrategischen Wirkungen untersucht werden können. Wie bereits aufgezeigt, liegt ein solches Grundmodell bisher nicht

1 Rockart, J.F., Short, J.E., Information Technology, a.a.O., S. 47. 
vor. Deswegen können aus dem Ziel der Arbeit drei Teilziele abgeleitet werden:

Erstens ist ein wettbewerbsstrategisches Grundmodell zu entwickeln, welches über die (statisch definierte) Analyse von Wettbewerbspositionen hinausgeht, so daß die skizzierten Zwangs- und Bindungswirkungen von Integrationsstrategien explizit in die Analyse einbezogen werden können. Dabei ist es von entscheidender Bedeutung, ein Gerüst mit Kriterien und Meßgrößen vorzulegen, das es erlaubt, die Dauerhaftigkeit von Wettbewerbspositionen abzuschätzen und das insoweit zeitablaufbezogen ist.

Zweitens muß ein Bezugsrahmen erarbeitet werden, der die Strukturelemente der strategischen Integrationsentscheidung enthält und diese zueinander in Bezug setzt. ${ }^{1}$ Dies sind, wie bereits diskutiert, die Integrationsinstrumente und die Integrationsbereiche, wobei letztere die Unternehmensteile und deren Verbindung durch vertikale Verknüpfungen und horizontale Verflechtungen umfassen. Weitere Strukturelemente der Integrationsentscheidung sind die situativen Bedingungen im Integrationsbereich und die Ziele der Integration. Unter Bezug auf den jeweiligen situativen Kontext im Integrationsbereich ist die Analyse der Zielwirkung des Einsatzes der Integrationsinstrumente zu ermöglichen. Dazu ist ein Gerüst von Kosten- und Nutzenkategorien der Integration zu erarbeiten. Diese sind auf das wettbewerbsstrategische Grundmodell, in dem eine Zielgröße der Integration zu entwickeln ist, zu beziehen.

Drittens ist auf Basis des Bezugsrahmens eine Taxonomie konkreter Integrationsstrategien zu entwickeln und deren Wirkung anhand des wettbewerbsstrategischen Grundmodells im einzelnen zu untersuchen.

1 Vgl. Kieser, A., Kubicek, H., Organisation, a.a.O. , S. 64, die vier Elemente eines handlungsorientierten Grundmodells des situativen Ansatzes angeben: Gestaltungsziele, Aktionsparameter der Gestaltung, situative Bedingungen und Ergebnisse der Gestaltung. 
Aus dem Ziel ergibt sich der Gang dieser Arbeit. Im Kapitel B wird ein Bezugsrahmen zur Integration von Geschäftsaktivitäten vorgelegt (zum folgenden vgl. Abb. A.6). Ausgangspunkt des Bezugsrahmens ist ein in Kapitel B.1 vorzulegendes zeitablaufbezogenes, wettbewerbsstrategisches Grundmodell, im folgenden als gebundener Wettbewerb bezeichnet. Aus diesem leitet sich die Zielgröße zur Beurteilung der Vorteilhaftigkeit von Integration ab: der dauerhafte Wettbewerbsvorteil, der neben der (statisch definierten) Wettbewerbsposition ihre Dauerhaftigkeit zum Schwerpunkt der Analyse macht.

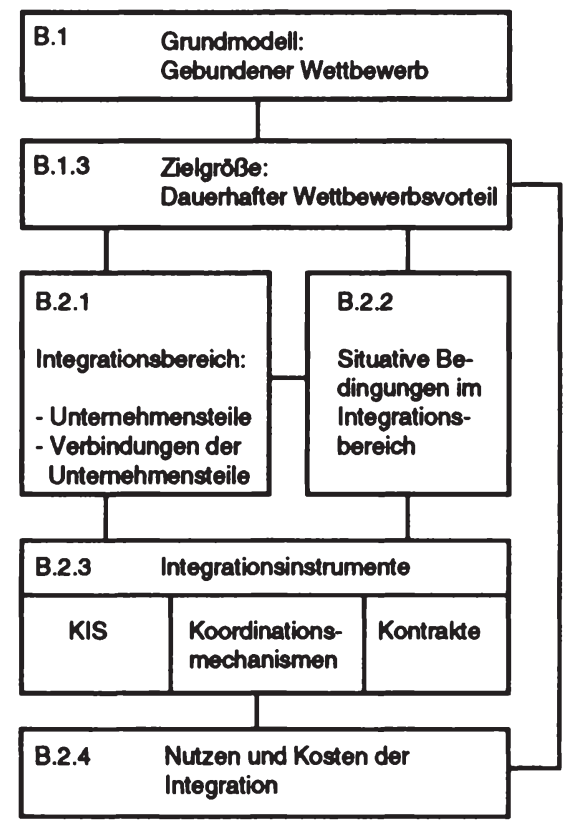

Abb. A.6: Bezugsrahmen mit Strukturelementen der Integrationsentscheidung

Kapitel B.2 geht dem zweiten Teilziel dieser Arbeit nach. Dazu werden die einzelnen Strukturelemente der Integrationsentscheidung konkretisiert und im Hinblick auf Kosten und Nutzen der Integration aufeinander bezogen. Erstes Strukturelement ist der Integrationsbereich (Abschnitt Nicolaus Henke - 978-3-631-75100-8 
B.2.1), der aus den die Geschäftsaktivitäten tragenden Unternehmensteilen besteht, welche mit vertikalen Verknüpfungen und horizontalen Verflechtungen integriert werden. Zweites Strukturelement sind die situativer Bedingungen im Integrationsbereich (Abschnitt B.2.2.), die die relative Vorteilhaftigkeit alternativ möglicher Integrationsstrategien mit bestimmen. Drittes Strukturelement sind die Integrationsinstrumente (Abschnitt B.2.3) KIS, Koordinationsmechanismen und Kontrakte ("3 K"). Viertes Strukturelement ist ein Gerüst von Kosten und Nutzen der Integration (Abschnitt B.2.4).

Dem dritten Teilziel, der Untersuchung der wettbewerbsstrategischen Wirkung einzelner Integrationsstrategien, wird in Kapitel $\mathrm{C}$ nachgegangen. Dazu wird eine Taxonomie von sechs Integrationsstrategien entwickelt. Diese lassen sich in Bezug auf das wettbewerbsstrategische Grundmodell einerseits und den Bezugsrahmen zur Integration andererseits unterscheiden. Die wettbewerbsstrategische Wirkung jeder dieser Integrationsstrategien wird in Bezug auf das eingangs entwickelte Grundmodell des gebundenen Wettbewerbs untersucht. Basis dieser Untersuchung sind 35 Fallstudien, die quer durch verschiedene situative Bedingungsrahmen, Integrationsinstrumente sowie völlig verschiedene Problemebenen analysiert werden sollen.

Im Kapitel D werden als Ergebnis die wettbewerbsstrategischen Wirkungen von Integrationsstrategien knapp zusammengefaßt und diskutiert, ob und unter welchen allgemeinen Bedingungen die Schaffung dauerhafter Wettbewerbsvorteile durch die Integration von Geschäftsaktivitäten, unter besonderer Berücksichtigung des Einsatzes von KIS, möglich ist oder nicht. 
B Grundmodell und Bezugsrahmen zur wettbewerbsstrategischen Analyse von Integrationsentscheidungen

Ausgangspunkt der Integrationsentscheidung sind die mit ihr verfolgten Ziele. Ziele lenken (Integrations)-Entscheidungen, indem die angenommenen Wirkungen der Entscheidungsalternativen mit den Forderungen des Ziels verglichen und damit beurteilbar gemacht werden.. ${ }^{1} \mathrm{Um}$ den Beitrag von Integrationsentscheidungen zum Unternehmensziel beurteilen $\mathbf{z u}$ können, sind die Zielinhalte zu klären. Heinen weist darauf hin, daß bei der Erklärung der Zielinhalte die dem Problem zugehörigen betriebswirtschaftlichen Grundmodelle eine entscheidende Rolle spielen. ${ }^{2}$ Ein solches ist für die strategische Integrationsentscheidung zu entwickeln. Der Zielbeitrag einer strategischen Entscheidung, also auch einer Integrationsentscheidung, kann anhand des Beitrags zum Wettbewerbsvorteil operationalisiert werden. ${ }^{3}$ Ein Wettbewerbsvorteil ist eine im Vergleich zu den Wettbewerbern überlegene Leistung der Unternehmung darin, Nutzen für den Kunden zu erbringen. 4

1 Vgl. Hauschildt, J., Zielsysteme, in: Handwörterbuch der Organisation, (Hrsg.), Grochla, E., 2. Aufl., Stuttgart, 1980, Sp. 2419.

2 Vgl. Heinen, E., Grundlage betriebswirtschaftlicher Entscheidungen: Das Zielsystem der Unternehmung, Wiesbaden, 1976, S. 14; Heinen, E., Einführung in die Betriebswirtschaftslehre, 9. Aufl., Wiesbaden, 1986, S. 99.

3 Vgl. Gluck, F.W., Strategic Choice and Resource Allocation, in: McKinsey Quarterly, Winter 1980, S. 23 ff; Porter, M.E., Competitive Advantage, a.a.O., S. 4 ff. Einen Überblick geben Meffert, H., Strategische Unternehmensführung, a.a.O., bes. S. 3 - 50; ders.: Marketing, a.a.O., S. 54 ff; Hax, A.C., Majluf, N. S., Strategic Management. An Integrative Perspective, Englewood Cliffs, 1984; Gluck, F.W., Kaufman, S.P., Walleck, A.S., Competitive Advantage, a.a.O.; Hinterhuber, H., Zur Objektivierung strategischer Entscheidungen, in: Informationstechnologie und strategische Führung, (Hrsg.), Spremann, K., Zur, E., Wiesbaden, 1989, S. 29 - 48; Ansoff, H.I., The New Corporate Strategy, 3rd ed., New York, 1988. Zur Unterscheidung der Konzepte der Wettbewerbsstrategie, der strategischen Unternehmensführung sowie der strategischen Planung vgl. Scholz, Ch., Strategisches Management, a.a.O., bes. S. 4 - 7; Hahn, D., Unternehmensplanung, a.a.O.; Meffert, H., Wertkette, a.a.O., S. 257 ff; Dannenberg, J., Miknocomputergestützte Instrumente, a.a.O., S. 16 ff; Overlack, J., Wettbewerbsvorteile, a.a.O., S. 31 ff; Ropella, W., Synergie als strategisches Ziel der Unternehmung, Berlin, New York, 1989, S. 27 ff.

4 Vgl. z.B. Aaker, D.A., Kriterien zur Identifikation dauerhafter Wettbewerbsvorteile, in: Wettbewerbsvorteile und Wettbewerbsfähigkeit, (Hrsg.), Simon, H., Stuttgart, 1988, S. 37. 


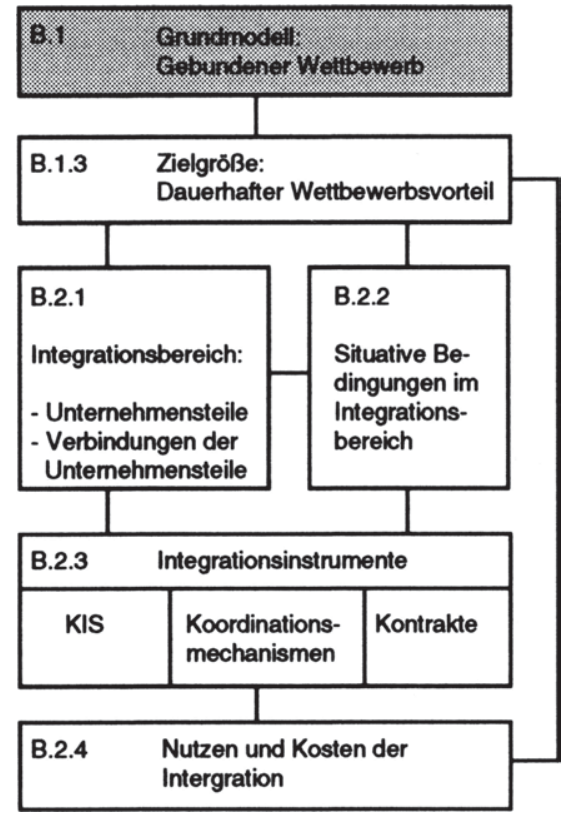

Abb. B.1.1: Stellung des Grundmodells im Bezugarahmen

Der dem Zielinhalt "Wettbewerbsvorteil" zugehörige wettbewerbsstrategische Ansatz von Porter ${ }^{1}$ ist vor dem Hintergrund des Zwangs- und Bindungscharakters von Integrationsstrategien zu erweitern. In der Literatur liegen erste Ansätze, die auch die Dauerhaftigkeit von Wettbewerbsvorteilen einbeziehen, zwar bereits vor. ${ }^{2}$ Diese enthalten zwar Kriterienlisten zur Beurteilung von Wettbewerbsvorteilen und deren Dauerhaftigkeit. Sie werden jedoch weder aus einem zeitablaufbezogenen Grundmodell hergeleitet und damit auf erklärende Faktoren zurückführt noch auf Integra-

1 Porter, M.E., Competitve Advantage, a.a.O.

2 Ansätze dazu finden sich bereits bei Coyne, K.P., Die Struktur dauerhafter Wettbewerbsvorteile, in: Wettbewerbsvorteile, (Hrsg.), Simon, H., a.a.O., S. 18 ff; Ghemawat, P., Dauerhafte Wettbewerbsvorteile aufbauen, in: Wettbewerbsvorteile, (Hrsg.), Simon, H., a.a.O., S. 30 - 36; sowie Aaker, D.A., Dauerhafte Wettbewerbsvorteile, in: Wettbewerbsvorteile, (Hrsg.), Simon, H., a.a.O., S. 37 - 46. 
tionsentscheidungen angewandt. Im folgenden Abschnitt wird das wettbewerbsstrategische Grundmodell des gebundenen Wettbewerbs herangezogen. Der zugehörige Zielinhalt ist der dauerhafte Wettbewerbsvorteil. (vgl. Abb. B.1.1).

Das Konstrukt des dauerhaften Wettbewerbsvorteils läßt sich durch das Caves'sche Konzept des gebundenen Wettbewerbs (Committed Competition) inhaltlich ausfüllen. ${ }^{1}$ Danach kann das strategische Problem der Unternehmung interpretiert werden als die Erzielung von Renten aus dem Einsatz eines partiell fixen Faktorbündels und die Komposition desselben. Die Zusammensetzung des Faktorbündels determiniert den Wettbewerbsvorteil. Dieses ist jedoch nicht beliebig veränderbar. Mitarbeiter und Vermögensgegenstände können nicht jederzeit ausgewechselt und die Art ihrer rechtlichen, organisationalen und technischen Integration nicht sofort verändert werden. Im Wettbewerb versucht die Unternehmung, ihre Zielfunktion unter der Restriktion des ihr gegebenen partiell fixen Faktorbündels zu maximieren. Je weniger veränderlich sowohl die Faktoren als auch deren Integration im Faktorbündel der Unternehmung sind, desto stärker sinken die (entscheidungsrelevanten) Grenzkosten des Faktoreinsatzes unter die Durchschnittskosten des Faktoreinsatzes. Können trotz der in die fixen Faktoren versunkenen Kosten Zielbeiträge realisiert werden, die über den Grenzkosten liegen, stellen diese eine ökonomische Rente dar. ${ }^{2}$ Wettbewerb schmelzt Renten ab. In einem Wettbewerbsumfeld setzt die Erzielung einer ökonomischen Rente aus einem Faktorbündel voraus, daß die Unternehmung einen Vorteil gegenüber ihren Wettbewerbern hat. ${ }^{3}$

1 Caves, R.E., Economic Analysis and the Quest for Competitive Advantage, in: American Economic Review, Vol. 74, 1984, S. 127 - 132.

2 Vgl. Caves, R.E., Economic Analysis, a.a.O., S. 130.

3 Vgl.Kapitel B.1.3. 


\subsection{Bindungswirkung von Commitments}

Strategische Entscheidungen sind Entscheidungen über die Komposition des Faktorbündels. Mit ihnen gehen Unternehmen Commitments (Bindungen) ein, die nicht oder kaum reversibel sind. Dies gilt z.B. für eine Investition in langfristig zu nutzende Faktoren, deren Veräußerung oder anderweitige Nutzung einen geringeren Wert erbrächte als die Nutzung für die Strategie, für die ihr Einsatz geplant ist.1 ${ }^{1}$ Mit einem eingegangenen Commitment, z.B. durch den strategischen Einsatz von KIS, legt sich ein Unternehmen auf eine bestimmte Integrationsstrategie fest. Ist ein $\mathrm{Ge}-$ schäftssystem erst einmal an die neue Integrationsstrategie angepaßt worden, wird es zunächst kaum möglich sein, dies rückgängig zu machen. Verschiedene Unternehmensteile sowie Lieferanten, Absatzkanal und Kunden haben sich Z.B. auf neue elektronische vertikale Verknüpfungen und horizontale Verflechtungen umgestellt. Damit wurde eine Bindung, ein Commitment, für einen großen Zeitraum eingegangen, über dessen Verlauf eine Amortisation der Ausgangsinvestition angestrebt werden muß.

Das Phänomen der Commitments ist in der Betriebswirtschaftslehre keineswegs neu. So sah schon Schmalenbach die Problematik der Bindungswirkung in seinem kontroversen Traktat vom Ende der freien Wirtschaftsverfassung: "Weil die proportionalen Kosten in so großem Umfange fix geworden sind, fehlt der Wirtschaft die Fähigkeit der Anpassung".2 Diese Bindungswirkung bzw. partielle Fixiertheit des Faktorbündels der Unternehmung kann auf drei Gründe zurückgeführt werden: ${ }^{3}$ starre Faktoren,

1 Vgl. Caves, R.E., Economic Analysis, a.a.O., S. 127.

2 Schmalenbach, E., Die Betriebswirtschaftslehre an der Schwelle der neuen Wirtschaftsverfassung, in: Zeitschrift für handelswissenschaftliche Forschung, 22. Jg., 1928, S. 245. Schmalenbach zielte aus damaliger Perspektive darauf ab, die Produktion an die Konsumption anzupassen und argumentiert mit fixen Kosten anstelle von fixen Faktoren (d.h. versunkenen Kosten). Die Logik seines Arguments trifft aber genau das hier diskutierte Problem: die Frage der Abhängigkeit von langfristig einzugehenden Commitments.

3 Nach Ghemawat, P., Commitment, The Dynamic of Strategy, Boston, 1991, S. 17, der jedoch vier Gründe unterscheidet: Lock-in, Lock-out, Lags und Inertia. Dieser Gliederung wird nicht gefolgt, um die hier eingeführten Dimensionen Basis und Art von Commitments zu unterscheiden sowie um die enge Verwandschaft von "Lags" und "Inertia" zu (Fortsetzung der Fußnote auf der nächsten Seite) 
entgangene Optionen und organisationale Trägheit (vgl. Abb. B.1.2). Der Unterschied zwischen diesen Gründen kann anhand der Basis und der Art von Commitments aufgezeigt werden. Die Basis eines Commitments kann ökonomisch, zeitlich oder psychologisch sein. Die Art eines Commitments kann die Unternehmung an eine einmal getroffene Entscheidung binden (Lock-in)1, z.B. nach Investitionen, oder Handlungsmöglichkeiten ausschließen (Lock-out), z.B. bei Nichtinvestition.

\begin{tabular}{|c|c|c|}
\hline Art & "Lock in" & "Lock out" \\
\hline "Okonomisch" & $\begin{array}{l}\text { Starro } \\
\text { Faktoren }\end{array}$ & $\begin{array}{l}\text { Entgangene } \\
\text { Optionen }\end{array}$ \\
\hline$\frac{\text { "zeitlich" }}{\text { "psychologisch" }}$ & \multicolumn{2}{|c|}{$\begin{array}{l}\text { Prozessualo organisationalo Trägholt } \\
\text { Strukturelb organisationalo Trägholt }\end{array}$} \\
\hline
\end{tabular}

Abb. B.1.2 : Gründe für die Bindungswirkung von Commitments

\subsubsection{Starre Faktoren}

Nahezu jede strategische Entscheidung bindet Ressourcen im Hinblick auf eine Strategie: "Commitments are long-lived and limited in resale value."2 Dies trifft in besonderem Maße auf Integrationsentscheidungen zu. Veränderungen von KIS, Koordinationsmechanismen und Kontrakten und die Abstimmung zwischen diesen setzen erhebliche monetäre und nichtmonetäre Investitionen voraus. Die eingesetzten Faktoren werden starr. Sie zahlen sich erst über einen langen Zeitraum aus. Die Starrheit von Faktoren beruht auf der zeitlichen Struktur der Zahlungen für diese. Abb. B.1.3 zeigt das Profil der Zahlungen einer Strategie gegenüber der Opportunitätsstra-

berücksichtigen.

1 Zum Lock-in vgl. Klein, B., Crawford, R., Alchian, A.A., Vertical Integration, Appropriable Rents, and the Competitive Contracting Process, in: Journal of Law and Economics, Vol. 21, 1978, S. 301.

2 Caves, R., Economic Analysis, a.a.O., S. 128. 
tegie im Zeitablauf, die Einzahlungen $B(\tau)$ sowie die Zahlungsausgänge $C(\tau)$ in jedem Zeitpunkt $\tau$ des Planungshorizonts T. Für die Auszahlungen $B(\tau)$ wurde dabei ein dem Verlauf eines Produktlebenszyklus ähnliches Profil angenommen, für die Zahlungsausgänge $C(\tau)$ beträchtliche Lern- bzw. Erfahrungskurveneffekte im Zeitablauf. ${ }^{1}$ Außerdem liegt ein Kostenvorlauf vor, d.h. in einem Zeitraum von $\tau=0$ bis $\tau=t$ sind Auszahlungen zu leisten, denen keine Einzahlungen entgegenstehen.

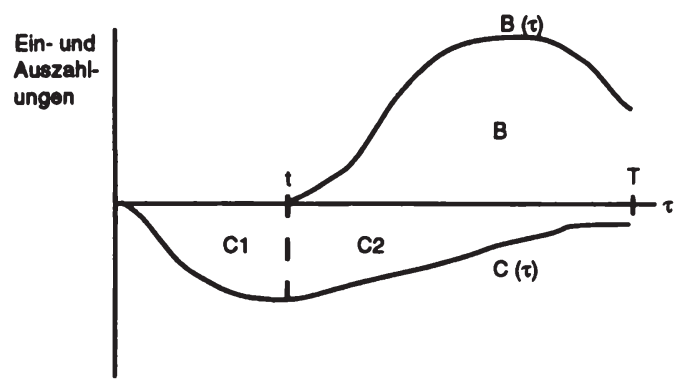

B.1.3: Zahlungsprofil einer Strategie mit Kostenvorlauf

Die Unternehmung entscheide am Ausgangspunkt $\tau=0$ darüber, ob die Strategie verfolgt werden soll. Ihr Entscheidungskriterium sei der Barwert NPV aller Zahlungen bei einer stetigen Verzinsung zum Zinssatz r:

$$
N P V(0)=\int_{0}^{T} C(\tau) e^{-\pi} d \tau+\int_{0}^{T} B(\tau) e^{-r \tau} d \tau \quad \text { (Flächen } \mathrm{C} 1+\mathrm{C} 2+\mathrm{B} \text { ) }
$$

Nachdem der Zeitraum von 0 bis $\mathbf{t}$ verstrichen ist, stellt sich der Barwert der Strategie dar als

$$
N P V(t)=\int_{t}^{T} C(\tau) e^{-r \tau} d \tau+\int_{t}^{T} B(\tau) e^{-r \tau} d \tau \quad \text { (Flächen } \mathrm{C} 2+\mathrm{B} \text { ) }
$$

1 In der Literatur finden sich vereinfachende, kumulierte Zahlungs-Profile von Strategien, z.B. bei Hinterhuber, H., Objektivierung, a.a.O., S. 43; oder bei House, Ch.H., Price, R.L., The Return Map: Tracking Product Teams, in: Harvard Business Review, Vol. 81, January February 1991, S. 94. Diese sind konsistent mit dem angegebenen Profil. 
Damit ist die Strategie zum Zeitpunkt $\tau=t$ gemessen am Barwert im Vergleich zum Ausgangszeitpunkt $\tau=0$ günstiger geworden um den versunkenen Betrag:

$$
N P V(t)-N P V(0)=\int_{0}^{1} C(\tau) e^{-r \tau} d \tau \quad \text { (Fläche C1). }
$$

Sollte die Unternehmung in $\tau=t$ die Strategie überprüfen, sind nur noch die Flächen C2 und B relevant. Wenn eine Neueinschätzung ergibt, daß entgegen der ursprünglichen Erwartung höhere Auszahlungen $C(\tau)$ oder geringere Einzahlungen $B(\tau)$ vorliegen werden, wird die Unternehmung die Strategie dennoch fortführen, zumindest falls der Barwert der Verschlechterung kleiner ist als der Barwert, der Fläche $\mathrm{C} 1$ entspricht. Ein Beispiel für eine solche Situation ist die Investition in ein Informationssystem. Sobald das System installiert ist, lohnen sich für einen langen Zeitraum nur inkrementale Verbesserungen, selbst wenn neue, bessere Systeme erworben werden könnten. Die bis zum Zeitpunkt $\tau=t$ getätigten Zahlungsausgänge (z.B. für Hardware, Software, Organisationsanpassungen, Schulung usw.) sind versunken, die damit erworbenen Faktoren starr.

Die Starrheit von Faktoren hängt damit von drei Einflußgrößen ab, erstens ihre Einsetzbarkeit für eine Strategie, nicht aber einer anderen, zweitens, der Höhe des Kostenvorlaufs (d.h. der versunkenen Kosten), drittens, der Höhe der anzusetzenden Verzinsung.

\subsubsection{Entgangene Optionen}

Entgangene Optionen beruhen auf versunkenen Opportunitätskosten vergangener Entscheidungen. Das oben angeführte Zahlungsprofil stellt die Differenz einer Strategie zur Opportunität dar. Dabei kann die Opportunität einer Nichtinvestition genau wie die Investition Kosten verursachen, nämlich Opportunitätskosten künftig entgangener Optionen aufgrund einer Nichtinvestition. Auch diese Opportunitätskosten versinken im Zeitablauf 
und sind dann Basis eines strategischen Commitments. Beispielsweise wird es kaum möglich sein, die Entscheidung nach einigen Jahren zu revidieren, einen zum Verkauf stehenden Zulieferer kontraktuell, technisch und organisatorisch in die Unternehmung $\mathrm{zu}$ integrieren. Andere Wettbewerber können der Unternehmung zuvorgekommen sein. Insofern zieht die Entscheidung Opportunitätskosten nach sich. Im genannten Beispiel sind dies die entgangenen Integrationsgewinne.

Solche Opportunitätskosten können bei Entscheidungen über das Timing eines Markteintritts entstehen sowie beim Einstieg in KIS auf Basis einer technologisch neuen Generation. So ist die Wahl des Eintrittszeitpunkts in einen Markt aus Sicht des Konzepts des gebundenen Wettbewerbs von hoher Bedeutung, ${ }^{1}$ da zum Zeitpunkt des Eintritts Investitionen in starre Faktoren entstehen, ein Nichteintritt jedoch Opportunitätskosten verursacht. Beide begründen Commitments, binden die Unternehmung für zukünftige Entscheidungen - sei es die Bindung an starre Faktoren oder die entgangene Option des Eintritts in einen Markt.

\subsubsection{Organisationale Trägheit}

Organisationale Trägheit beruht auf der begrenzten Wandlungs- und Lernfähigkeit der Unternehmung. ${ }^{2}$ Organisationale Trägheit kann auf zwei Faktoren zurückgeführt werden: die prozessuale Trägheit und die strukturelle Trägheit. ${ }^{3}$ Die prozessuale Trägheit bezieht sich auf die Handlungsschnelligkeit, mit der Geschäftsaktivitäten ausgeführt werden. Einer Erhöhung der Handlungsgeschwindigkeit setzen nicht nur die technischen Bedingun-

1 Zur Bedeutung des Timings von Markteintrittsentscheidungen vgl. Meffert,H., Strategische Unternehmensführung, a.a.O., S. 63.

2 Vgl. Frese [1991] S. 13.

3 Meffert unterscheidet konsistent dazu im Zusammenhang mit der Flexibilität die Prozeßflexibilität als Handlungsgeschwindigkeit und die Strukturflexibilität als Handlungsbereitschaft. Vgl. Meffert, H., Größere Flexibilität als Unternehmenskonzept, in: Zeitschrift für betriebswirtschaftliche Forschung, Vol. 37, 1985, S. $123 \mathrm{ff}$. 
gen Grenzen, sondern auch gegenseitige Abhängigkeiten, welche sich aus der erforderlichen logischen Reihung von Geschäftsaktivitäten ergeben.

Die strukturelle Trägheit bezieht sich auf die Bereitschaft von Organisationen zu handeln und der in Organisationen arbeitenden Individuen, Anpassungen vorzunehmen. Zahlreiche verhaltenswissenschaftliche Ansätze beschreiben psychologische Mechanismen, die individuelle wie kollektive Verhaltensänderungen begrenzen, selbst wenn verhaltensänderndes Lernen angestrebt wird. ${ }^{1}$ Das Beharrungsvermögen von Organisationen, die ihnen innewohnende Neigung zum Status quo, kann auf diese psychologischen Mechanismen zurückgeführt werden. Angesichts begrenzter Rationalität des Menschen können auch das Wissen und die Fertigkeiten des Menschen als "starre Faktoren" angesehen werden, die durch Lernen nur in Grenzen verändert werden können.

Organisationale Trägheit verstärkt die Bindungswirkung von Commitments nicht nur direkt, sondern auch indirekt - durch die Erhöhung der Starrheit von Faktoren und der entgangenen Optionen. Die prozessuale Trägheit verzögert zusätzlich die Fertigstellung eines (aus mehreren Teilprozessen bestehenden) Vorgangs. Die Vorteilhaftigkeit einer Strategie kann aber oft erst dann beurteilt werden, wenn ein gesamter Vorgang abgeschlossen ist. So wird eine Produktneuentwicklung abschließend erst nach Markteinführung, zumindest nach Markttests, beurteilt werden können. Je später dieser Zeitpunkt, desto stärker werden für die gewählte Strategie starre Faktoren gebildet bzw. alternative Möglichkeiten ausgeschlossen. Damit sinkt durch Verzögerung die Wahrscheinlichkeit, einmal getroffene Entscheidungen zu revidieren - d.h. die Bindungswirkung von Commitments nimmt zu.

1 Vgl. z.B. Argyris, Ch., Overcoming Organizational Defenses, Needham Heights, 1990, S. 12 ff; Cyert, R.M., March, J.G., A Behavioral Theory of the Firm, Englewood Cliffs, New Jersey, 1963; Levitt, B., March, J.G., Organizational Learning, in: Annual Review of Sociology, Vol. 14, 1988, S. 320; Fiol, C.M., Lyles, M.A., Organizational Learning, in: Academy of Management Review, Vol. 10, No. 4, 1985, S. 804 f; Janis, I., Victims of Groupthink, Boston, 1972; Frese [1991] S. 13; Meffert, H., Größere Flexibilität als Unternehmenskonzept, a.a.O., S. $130 \mathrm{ff}$ und dort angegebene Literatur. 
Aus ökonomischer Sicht sollten diese versunkenen Kosten weitere Entscheidungen nicht beeinflussen. Verschiedene Autoren ${ }^{1}$ haben jedoch gezeigt, daß versunkene Kosten über psychologische Faktoren durchaus auf tatsächliches menschliches Verhalten wirken. Die versunkenen Kosten für eine vergangene Entscheidung reduzieren danach die Wahrscheinlichkeit, jene zu revidieren. Dies gilt auch, wenn die Entscheidung sich als falsch herausstellt und eine Revision vorteilhaft wäre. Diese in der "Psychology of Sunk Cost" als Bedauern oder Enttäuschung bezeichneten psychologischen Faktoren erhöhen die strukturelle Trägheit einer Organisation.

Die Bindungswirkung von Commitments ist dabei nicht "unerwünscht". Langfristige Profitabilität setzt geradezu Commitments voraus, denn es bedarf langfristiger und sich kumulierender Anstrengungen, eine Wettbewerbsposition aufzubauen. Strategische Commitments müssen eingegangen werden, um die Unternehmung überhaupt in die Lage zu versetzen, Renten aus dem Einsatz des ihr verfügbaren Faktorbündels, beispielsweise aus starren KIS, Produktionsanlagen oder Markenprofilierung, zu schöpfen. Die Fixierung von Faktoren ist ein Grund dafür, diese Faktoren innerhalb der Unternehmung zu bündeln anstatt sie extern vom Markt zu beziehen. Am Spotmarkt können nur kurzfristige Gewinne durch Arbitrage erzielt werden, die unmittelbarer Imitation ausgesetzt sind.

1 Bell, D.E., Disappointment in Decision Making under Uncertainty, in: Operations Research, Vol. 33, 1985, S. 27; Bell, D.E., Regret in Decision Making under Uncertainty, in: Operations Research, Vol. 30, 1982, S. 961; Loomes, G., Sugden, R., Regret Theory, in: Economic Journal, Vol. 92, 1982, S. 805 - 824; Arkes, H.R., Blumer, C., The Psychology of Sunk Cost, in: Organization Behavior and Human Decision Processes, Vol. 35, 1985, S. 124 -140 . 


\subsection{Notwendigkeit zeitablaufbezogener Analyse und vorausschauender Planung}

Zu jedem Zeitpunkt steht einer Unternehmung und ihren aktuellen und potentiellen Konkurrenten ein Bündel aus partiell gebundenen Faktoren zur Verfügung. Das strategische Problem der Unternehmung ist es, einerseits die gegenüber der Konkurrenz möglichen Renten aus dem partiell fixen Faktorbündel zu maximieren, andererseits durch ständiges Lernen die Komposition des partiell fixen Faktorbündels so $z u$ verändern, daß ein Wettbewerbsvorteil geschaffen und dauerhaft erhalten werden kann. Der Anpassung des Faktorbündels an neue Bedingungen sind Grenzen gesetzt. Unternehmungen können Strategien nicht beliebig ändern, sie sind vielmehr durch in der Vergangenheit getroffene Entscheidungen gebunden. Dies kann, Ghemawat ${ }^{1}$ folgend, anhand folgender Matrix illustriert werden.

Strategie zum Zeitpunkt $t+\Delta t$

A

B

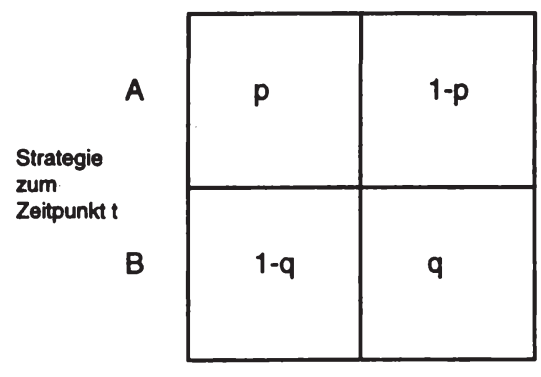

Abb. B.1.4: Übergangswahrscheinlichkeiten zwischen Strategien

Quelle: Ghemawat, P., Commitment, Boston 1991, S. 15

Eine Unternehmung könne zwischen zwei alternativen Strategien wählen, $A$ und B. Dabei gebe $p$ (bzw. $q$ ) ist die Wahrscheinlichkeit an, daß zu einem Zeitpunkt $t+\Delta t$ Strategie A (bzw. B) beibehalten wird, wenn A (bzw. B)

1 Ghemawat, P., Commitment, The Dynamic of Strategy, Boston, Massachusetts, 1991. 
auch zum Zeitpunkt $t$ bestand. Entsprechend bezeichnet die Gegenwahrscheinlichkeit 1 - p (bzw. 1-q) einen Wechsel zur Strategie B (bzw. A) zum Zeitpunkt $t+\Delta t$. (vgl. Abb. B.1.4)Ein Commitment liegt vor, wenn es wahrscheinlicher ist, daß die Unternehmung in $t+\Delta t$ bei ihrer in $t$ gewählten Strategie bleibt, d.h. wenn $p>1$ - $q$ bzw., äquivalent dazu $q>1$ - $p$ ist.

Diese sehr einfachen Grundaussagen gewinnen erhebliche Bedeutung bei der Betrachtung der Dynamik des Wettbewerbs. Ohne Commitment könnte eine Unternehmung beliebig von einem Ist- auf ein Sollprofil wechseln. Commitments binden Unternehmungen an ihre vergangenen Entscheidungen. Die Folge von $\mathrm{A}$ auf $\mathrm{A}$ wird wahrscheinlicher als die Folge von $\mathrm{B}$ auf $A$, die Folge von $B$ auf $B$ wahrscheinlicher als die Folge von B auf $A$. Deshalb machen diese Bindungen eine zeitablaufbezogene Analyse und vorausschauende Planung erforderlich.

Arrow zeigt dies, indem er das Optimalverhalten der Unternehmung für den Fall untersucht, daß jede (Investitions-)Entscheidung reversibel wäre, 1 somit keine Commitments vorlägen. Dann wäre es optimal, genau bis zu jenem Punkt Investitionen vorzunehmen, bis zu dem das MomentanGrenzprodukt des Kapitals den Momentan-Grenzauszahlungen gleicht. Damit ist in jedem Zeitinkrement der optimale Kapitalstock "myopisch", d.h. unabhängig von zukünftigen Erwartungen, z.B. bezüglich technologischer Entwicklungen, Nachfrageverschiebungen oder Angebotsveränderungen. Ohne die Existenz von Commitments, also bei vollständiger Handlungsflexibilität, ${ }^{2}$ wäre eine langfristig orientierte, strategische Unternehmensführung nicht erforderlich. Vielmehr könnte in jedem beliebig

1 Arrow, K.J., Optimal Capital Policy, the Cost of Capital, and Myopic Decision Rules, Annals of the Institute of Statistics and Mathematics, Tokyo, Vol. 16, 1964, S. 21 - 30.

2 Vgl. Meffert, H., Größere Flexibilität als Unternehmenskonzept, a.a.O., S. 125 f; Hax, H., Laux, H., Flexible Planung - Verfahrensregeln und Entscheidungsmodelle für die Planung bei Ungewißheit, in: Schmalenbachs Zeitschrift für betriebswirtschaftliche Forschung, 24. Jahrgang, 1972, S. 319. Das Gegenteil von Commitments ist die vollständige Handlungsflexibilität. Diese ist nur in Extremsituationen gegeben, folglich liegen Commitments vor. (Handlungs-)Flexibilität kann im Rahmen einer vorausschauenden Planung explizit vorgesehen werden. 
kurzen Zeitraum das Faktorbündel der Unternehmung an die Erfolgssituation vollständig angepaßt werden. Beispielsweise würde ein Unternehmensteil sofort veräußert oder seine strategische Position verändert, sobald er einmal Periodenverluste aufwiese. Andererseits unterblieben Investitionen, steigerten sie nicht sofort die Profitabilität.

Unternehmungen können aber ihr Faktorbündel nicht in solcher Weise verändern. Formal ausgedrückt gilt $p>1-q$. Strategische Entscheidungen sind partiell irreversibel. Dies gilt auch, wenn Organisationen gelegentlich ihr Beharrungsvermögen überwinden und dann fundamentale, umfassende Wandelsprozesse durchschreiten. Diese empirisch beobachtete Tatsache ${ }^{1}$ bildet die in Abb. B.1.4 dargestellte dichotome Matrix genau ab: Aufgrund strategischer Commitments neigen Unternehmungen zwar überwiegend (mit p bzw. q) zur Beibehaltung einmal gewählter Strategien. Entscheidet man sich jedoch anders - mit der (kleineren) Gegenwahrscheinlichkeit 1-p bzw. 1-q, bedeutet dies einen "Schwenk" auf eine fundamental andere Strategie. ${ }^{2}$ Diese begründen neue Commitments mit langfristiger Bindung und erfordern damit ihrerseits eine zeitablaufbezogene Analyse und vorausschauende Planung.

\subsection{Dauerhafter Wettbewerbsvorteil}

als Zielgröße von Integrationsentscheidungen

Commitments schaffen also die Notwendigkeit vorausschauender Planung, die dazu dient, ein partiell fixes Faktorbündel so zu gestalten, daß die Rente daraus maximiert wird. Mit diesem Konzept des gebundenen Wettbewerbs und seinem zentralen Konstrukt, den Commitments, liegt ein betriebswirtschaftliches Grundmodell vor, auf dessen Basis die Bindungswirkung von Integrationsentscheidungen analysiert werden kann.

1 Vgl. z.B. Mintzberg, H., Patterns in Strategy Formation, in: Management Science, 1978 S. 934 - 948; Frese [1991] S. 13 und dort angegebene Verweise.

2 Vgl. Ghemawat, P., Commitment: The Cause of Stratetgic Persistence, Boston, (Fortsetzung der Fußnote auf der nächsten Seite) 
Ein Modell zur Analyse des Zielbeitrages von Integrationsentscheidungen kann mit dem Konstrukt des dauerhaften Wettbewerbsvorteil als zeitablaufbezogene Zielgröße strategischer Entscheidungen auf dieser Basis abgeleitet werden (vgl. Abb. B.1.5).

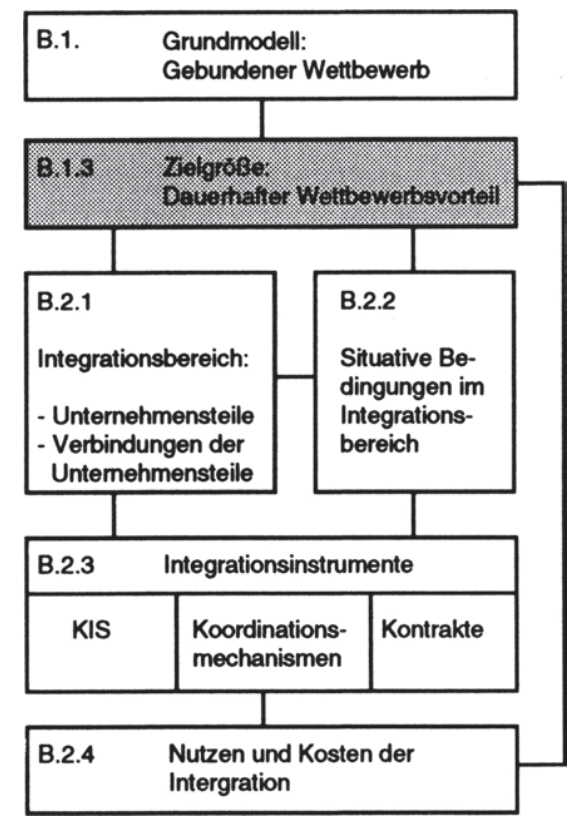

Abb. B.1.5: Stellung des dauerhaften Wettbewerbsvorteils im Bezugsrahmen

Die Schaffung von dauerhaften Wettbewerbsvorteilen kann als die Zielgröße der strategischen Unternehmensführung angesehen werden. Dabei steht der Wettbewerbsvorteil als eher heuristisches Sachziel in wechselseitiger, komplementärer Wirkungsbeziehung zu formalen Oberzielen der erwerbswirtschaftlichen Unternehmung, beispielsweise der langfristigen Gewinnerzielung, der Maximierung des Anteilswerts (als Wert der ausstehenden Aktien) oder der Maximierung des Unternehmenswerts (als Bar- 
wert der erwarteten künftigen Cash Flows). ${ }^{1}$ Diese Wirkungsbeziehung kann am Beispiel des Ziels der Gewinnerzielung anhand des Begriffs des Erfolgspotentials von Gälweiler aufgezeigt werden. Dauerhafte Wettbewerbsvorteile ermöglichen die nachhaltige Gewinnerzielung. Als Maßgröße des Potentials einer Unternehmung, nachhaltig Gewinn zu erzielen, schlägt Gälweiler das Erfolgspotential vor. ${ }^{2}$ Das Erfolgspotential ist die höchste (und langfristigste) Stufe in einem dreistufigen Konzept zur Unternehmenssicherung. Die erste Stufe umfaßt die Liquiditätssicherung im Rahmen der kurzfristigen Finanzplanung. Die zweite Stufe enthält die (mittelfristige) Erfolgssicherung der Unternehmung. Dabei fallen die Zeithorizonte von Liquiditäts- und Erfolgsplanung auseinander wie auch die korrespondierenden Maßgrößen Ausgaben und Einnahmen sowie Aufwand und Ertrag. Gleichwohl bilden erfolgsorientierte Entscheidungen die Grundlage der Liquiditätssicherung. Deswegen ist die Betrachtung des Erfolgs somit eine Betrachtung des Liquiditätspotentials. Die dritte Stufe umfaßt die noch längerfristigen strategischen Entscheidungen, die den (Perioden-)Erfolgsentscheidungen vorgelagert sind. Insofern kann die zugehörige Steuerungsgröße als Erfolgspotential bezeichnet werden. Aus der konsistenten begrifflichen Herleitung findet das Konzept des Erfolgspotentials gleichwohl noch keine operationale inhaltliche Ausfüllung. Dies ist unter Rückgriff auf das Konzept des gebundenen Wettbewerbs möglich. Erfolgspotential ist die Fähigkeit einer Unternehmung, aus dem ihr gegebenen Faktorbündel eine ökonomische Rente zu erzielen.

Aus dem Vergleich des Faktorbündels der Unternehmung mit denjenigen der Konkurrenten, jeweils bezogen auf das Kundenproblem, definiert sich die Wettbewerbsposition der Unternehmung. Sie läßt sich untergliedern in

1 Vgl. z.B. Heinen, E., Betriebswirtschaftslehre, a.a.O., S. 106; Copeland, T., Koller, T., Murrin, J., Valuation, Measuring and Managing the Value of Companies, New York, 1990, S. $73 \mathrm{ff}$.

2 Vgl. zum folgenden Gälweiler, A., Unternehmensplanung, Grundlagen und Praxis, Frankfurt, New York, 1974, S. 230; Gälweiler, A., Unternehmenssicherung und strategische Planung, in: Zeitschrift für betriebswirtschaftliche Forschung, 28. Jg., 1976, S. 363 ff; Dannenberg, J., Mikrocomputergestützte Instrumente, a.a.O., S. 24 ff. 
die Qualitätsposition, die Kostenposition und die Zeitposition (vgl. Abb. B.1.6).

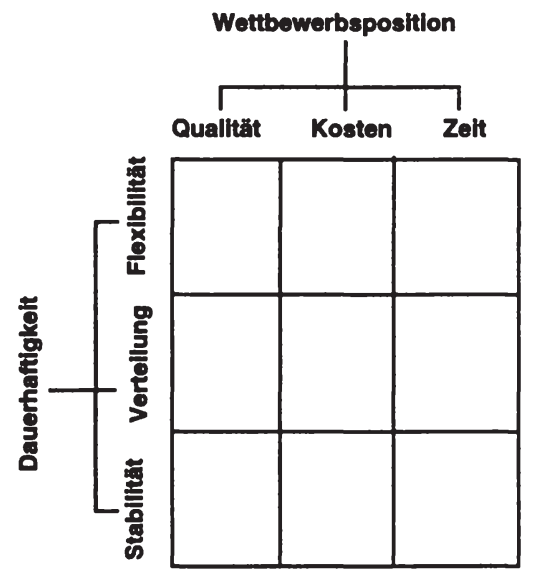

Abb. B.1.6: Einflußfaktoren auf den dauerhaften Wettbewerbsvorteil

Die Wettbewerbsposition bestimmt das Ausmaß eines Wettbewerbsvorteils zu einem Zeitpunkt. Angesichts der Bindungswirkung von Commitments ist die Betrachtung der Wettbewerbsposition zwar notwendig, jedoch nicht hinreichend. Zusätzlich ist die Dauerhaftigkeit eines Wettbewerbsvorteils zu betrachten. ${ }^{1}$ Dies ist die Zeitdauer, über die aus dem Wettbewerbsvorteil Rente erzielt wird. Die Dauerhaftigkeit hängt von drei Einflußfaktoren ab: der Stabilität der Wettbewerbsposition (z.B. gegenüber Imitation durch Konkurrenten), ihrer Flexibilität durch Offenhalten strategischer Handlungsoptionen sowie der Verteilung der erzielten Rente im Geschäftssystem (z.B. angesichts monopolistischer Abschöpfung durch den Absatzkanal) (vgl. Abb. B.1.6).

1 Vgl. dazu im einzelnen Abschnitt B.1.3.2. 


\subsubsection{Wettbewerbsposition}

Die Analyse der Wettbewerbsposition steht seit langem im Mittelpunkt von Ansätzen der strategischen Unternehmensführung ${ }^{1}$ und wird deswegen im folgenden nur knapp systematisiert.

\subsubsection{Qualitätsposition}

Die Qualität eines Produkts oder einer Leistung eines Unternehmens determiniert maßgeblich den Kundennutzen und ist damit wesentlich für die Erlangung eines Wettbewerbsvorteils. Gemäß Porter basieren Differenzierungsstrategien auf dem Angebot von Produkten oder Leistungen, die für den Kunden einen vergleichsweise einzigartigen Wert haben.2 Gälweiler sieht im "Anwenderproblem" die entscheidende strategische Orientierungsgröße. ${ }^{3}$ Meyer bezeichnet Qualität, definiert als Kundeneinschätzung des von der Unternehmung angebotenen Produkts, als "schärfste Wettbewerbswaffe" der Unternehmung. ${ }^{4}$

Ausgehend vom Kundenproblem, kann die relative Qualitätsposition der Unternehmung aus zwei Richtungen analysiert werden: die Kaufkriterien (Perspektive des Kunden) und die Lösungstechniken (Perspektive des Unternehmens) ${ }^{5}$ (vgl. Abb. B.1.7).

1 Vgl. Porter, M.E., Competitive Advantage, a.a.O., Kap. 2 - 3; vgl. Meffert, H., Marketing, a.a.O., S. 59 ff.

2 Porter, M.E., Competitive Advantage, a.a.O., S. $120 \mathrm{ff}$.

3 Gälweiler, A., Die strategische Führung der Unternehmung, in: Sonderdruck des Management-Zentrums St. Gallen, 1980, S. 20.

4 Meyer, J., Qualität als strategische Wettbewerbswaffe, in: Wettbewerbsvorteile und Wettbewerbsfähigkeit, (Hrsg.), Simon, H., 1988, S. 74.

5 Ein denkbarer dritter qualitätsbestimmender Faktor sind die verschiedenen und konfliktäre Anforderungen, Kunde und Absatzkanal an ein Produkt. Vgl. Meffert, H., Strategische Unternehmensführung, a.a.O., S. 48 ff; Porter, M.E., Competitive Advantage, a.a.O., S. 148. 


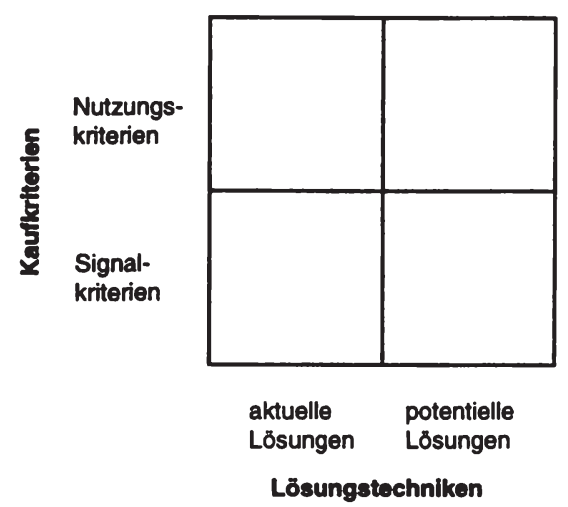

Abb. B.1.7: Qualitătsbestimmende Faktoren

Die Erfüllung der Kaufkriterien, d.h. inwieweit das Angebot einer Unternehmung das Kundenproblem löst, bildet des Kunden Maßstab zur Beurteilung der Qualität. ${ }^{1}$ Dabei sind Nutzungs- und Signalkriterien zu unterscheiden.

Nutzungskriterien sind die Vorteile, die der Kunde von einer Problemlösung erwartet. Dies sind entweder die Verbesserung der Funktionalität oder die Senkung seiner Kosten. ${ }^{2}$ Dem liegt die Betrachtung des tatsächlichen Nutzungsprozesses zugrunde. Beispielsweise kann ein elektronisches Warenbestellsystem die Lager- und Verwaltungskosten des Kunden senken. Durch ein elektronisches Fernüberwachungssystem wartungsanfälliger In-

1 Vgl. z.B. Porter, M.E., Competitive Advantage, a.a.O., S. 120; Siegwarth, H., Overlack, J., Langfristiger Erfolg durch Qualitätsstrategien, in: Harvard Manager, Heft 3, 1986, S. 65; Meyer, J., Strategische Wettbewerbswaffe, a.a.O., S. 74; Wittek, B., Strategien auf Kundennutzen aufbauen, in: Wettbewerbsvorteile und Wettbewerbsfähigkeit, (Hrsg.), Simon, H., Stuttgart, 1988, S. 66. In der Literatur wird verschiedentlich bemängelt, da $B$ im Zusammenhang mit der Betrachtung von Wettbewerbsvorteilen der Konkurrenzsituation zu große Beachtung gewidmet wird zu Lasten des eigentlichen Kundenproblems. Vielmehr seien nicht interne Ersatzkriterien und technische Spezifikationen, sondern die externe kundenbezogene Sichtweise entscheidend. Vgl. Wittek, B., Kundennutzen, a.a.O., S. 69; Overlack, J., Wettbewerbsvorteile, a.a.O., S. 210; Meyer, J., Strategische Wettbewerbswaffe, a.a.O., S. 75. Die Qualitätsposition der Unternehmung definiert sich jedoch nach der angebotenen Lösung des Kundenproblems im Vergleich am Angebot der Konkurrenten. Vgl. z.B. Porter, M.E., Competitive Advantage, a.a.O., S. 119, S. 138 ff. Dabei ist das Kundenproblem der Ausgangspunkt der Analyse, nicht aber deren einziger Inhalt.

2 Vgl. hierzu und zum folgenden Porter, M.E., Competitive Advantage, a.a.O., S. 135 ff. 
vestitionsgüter kann deren Funktionsfähigkeit verbessert und damit der Kundennutzen erhöht werden. Nutzungskritierien ergeben sich also aus den Anforderungen des Kunden an die Nutzung des Produkts. Porter weist darauf hin, daß ein "objektiver" Kundennutzen, gemessen an Nutzungskriterien, nicht notwendigerweise auch vom Kunden wahrgenommen wird. Dies kann auf mangelnde Signalkriterien zurückgeführt werden. Signalkriterien determinieren die Kundenwahrnehmung, ob die Nutzungskriterien durch ein Produkt oder eine Leistung tatsächlich erfüllt werden. ${ }^{1}$ Beispielsweise signalisiert die Benutzung eines elektronischen Flugreservierungssystems eine besondere Verläßlichkeit und Genauigkeit der Reservierung schon allein durch eine ansprechend gedruckte schriftliche Bestätigung anstelle einer handschriftlichen oder mündlichen Zusage. ${ }^{2}$ Dies gilt selbst bei hoher Überbuchung, wenn die Wahrscheinlichkeit einer sicheren Reservierung tatsächlich gering ist.

Nutzungskriterien und Signalkriterien sind definiert in Bezug auf das Kundenproblem. ${ }^{3}$ Gälweiler betont dabei die Notwendigkeit, das Kundenproblem lösungsinvariant, d.h. unabhängig von derzeit möglichen Lösungstechniken, hinreichend präzise zu formulieren und damit potentielle, zukünftige Lösungstechniken einzubeziehen. ${ }^{4}$ Dazu sollte, Porter folgend,

1 Methoden zur praktischen Erhebung von Nutzungs- und Signalkriterien des Kunden liegen in der Literatur vielfach vor, insbesondere in der Literatur strategischer Erfolgsfaktoren. Vgl. z.B. Daniel, R.D., Management Information Crisis, in: Harvard Business Review, Vol. 39, September, October 1961; Bullen, Ch.V., Rockart, J.F., A Primer On Critical Success Factors, Working Paper No. 69, Center for Information Systems Research, Sloan School of Management, MIT, Cambridge, Massachusetts, June 1981; Boynton, A.C., Shank, M.E., Zmud, R.W., Critical Success Factor Analysis as a Methodology for MIS Planning, in: MIS Quarterly, Vol. 9, 1985. Einen Überblick geben Rockart, J.F., De Long, D., Executive Support Systems, Homewood, Illinois, 1988, S. 201 ff; Overlack, J., Wettbewerbsvorteile, a.a.O., S. $180 \mathrm{ff}$.

2 Gleichwohl könnte eine solche Verläßlichkeit auch durch andere Signalkriterien erreicht werden, etwa die Reputation der Agentur. Eine Liste von Signalkriterien legt vor Porter, M.E., Competitive Advantage, a.a.O., S. 144.

3 Vgl. hierzu und zum folgenden Gälweiler, A., Strategische Führung, a.a.O., S. 20 f.

4 In diesem Zusammenhang kann freilich das Kundenproblem nicht lösungsinvariant formuliert und die Frage beantwortet werden: "Wer ist der Kunde?" Für eine sinnvolle Diskussion des Anwenderproblems muß das Segmentierungsproblem gelöst sein, wie für eine sinnvolle Diskussion des Segmentierungsproblems das Anwenderproblem vereinfachend kategorisiert werden muß. Eine solche Kategorisierung ist Abells dreidimensionales Vorgehen (Märkte, Produkte, Technologien). In der Systematik dieser Arbeit wird das Segmentierungsproblem insofern ausgeklammert und als gelöst angesehen, als es den Fra(Fortsetzung der Fußnote auf der nächsten Seite) 
der Anwendungs- oder Nutzungsprozess des Kunden anhand dessen Wertschöpfungskette analysiert werden. Die Analyse darf sich jedoch nicht auf die Kundenperspektive beschränken. Kundennutzen kann auch durch die (technische oder organisationale) Integration der Geschäftsaktivitäten von Kunde und Anbieter geschaffen werden. So kann eine Bank von ihren Firmenkunden elektronisch überspielte Daten entgegennehmen oder ihnen Zugang zum eigenen Auslandsinformations- und Transaktionssystem einräumen.

Nicht nur die Lösungstechniken unterliegen einem Wandel im Zeitablauf. Dies gilt ebenfalls für die Kaufkriterien und das Kundenproblem selbst. Für die Unternehmung ist dies nicht unproblematisch, da die Erreichung einer Qualitätsposition langwierige, sich kumulierende Anstrengungen, d.h. Commitments, erfordert. 1

Die Analyse der Kaufkriterien des Kunden und der Lösungstechniken reichen alleine zur Identifikation und Definition der relativen Qualitätsposition der Unternehmung nicht aus. Denn der Kunde wird bei gleicher Qualität das Angebot wählen, das seine "Konsumentenrente" maximiert, die sich als Überschuß des (kundenspezifisch definierten) Nutzens über dem Preis

gen der strategischen Integration vorgelagert ist. Entscheidungen etwa über die Integration von Unternehmensteilen mit Teilen der Kunden- oder Lieferantenunternehmung müssen von konkreten (rechtlichen) Personen ausgehen; sie können sich kaum auf abstrakte Segmentierungsfelder stützen. Zum Segmentierungsproblem vgl. Abell, D.F., Defining the Business, The Starting Point of Strategic Planning, Englewood-Cliffs, New Jersey, 1980; Meffert, H., Marketing, a.a.O., S. 243 ff.

4 Commitments bilden die wesentliche Basis der Schaffung von Qualität. Hierfür sprechen z.B. die Ergebnisse empirischer Messungen von Meyer im Rahmen des PIMS-Programms: Erstens weisen die Direktkosten keine Korrelation mit der von PIMS definierten relativen Qualität auf, zweitens verringern sich durch Qualitätsverbesserungen die relativen Einzelkosten. Danach kann argumentiert werden, daß bei Qualitätsstrategien eine Verlagerung von Einzel- in Gemeinkosten, von variablen in fixe Kosten vorgenommen wird. Beispielsweise zieht eine "flexible" Fertigungsanlage keineswegs eine elastische Kostenfunktion nach sich. Die Flexibilität einer flexiblen Fertigunganlage bezieht sich auf die Qualität, die Art der Ausbringung, nicht aber auf die Kosten. So weist auch Wildemann darauf hin, daß flexible Produktionstechniken die Fixkosten erhöhen. Wildemann, H., Erfolgspotentialaufbau durch neue Produktionstechnologien, in: Wettbewerbsvorteile, (Hrsg.), Simon, H., a.a.O., S. 122. Die Bindungswirkung dieser Fixkosten ist anhand der Faktoren prüfbar, die strategische Commitments verursachen: Erhöhung der Starrheit , Ausschluß von Optionen und Erhöhung der organisationalen Trägheit. Vgl. auch Horváth, P., Mayer, R., Prozeßkostenrechnung, in: Controlling, Bd. 1, Nr. 4, 1989, S. 215. (Fortsetzung der Fußnote auf der nächsten Seite) 
ergibt. Dies wird häufig anhand einer zweidimensionalen Matrix mit den Dimensionen "relativer Preis" und "relative Qualität" veranschaulicht. ${ }^{1}$

\subsubsection{Kostenposition}

Zur Analyse der Kostenposition hat sich in der Literatur zum strategischen Kostenmanagement ein schrittweises Vorgehen durchgesetzt. ${ }^{2}$ Ausgangspunkt ist die Untergliederung der Unternehmung in die einzelnen Wertaktivitäten ${ }^{3}$ und die Zuordnung der Kosten auf diese. Als Kriterien für diese Untergliederung nennt Porter (1) die Bedeutung (Size) und das Wachstum der für die Wertaktivität anfallenden Kosten und Aktiva, (2) das Kostenverhalten der Wertaktivität und (3) die Unterschiede zur Konkurrenz. ${ }^{4}$ Auf die so differenzierten Wertaktivitäten werden alle Kosten, insbesondere auch die fixen Kosten, und alle Aktiva zugeordnet, allerdings aufgrund des offensichtlichen Zurechnungsproblems nur sehr grob. 5

Zur Bildung von Commitments durch Kostenvorlauf vgl. Abschnitt B.1.1.

1 Auf eine Detaillierung wird hier verzichtet, vgl. dazu z.B. Buzzell, R.D., Gale, B.T., Das PIMS-Programm. Strategien und Unternehmenserfolg, Wiesbaden, 1989, S. 103 ff, bes. S. 112; Meyer, J., Strategische Wettbewerbswaffe, a.a.O., S. 80 f.

2 Vgl. Porter, M.E., Competitive Advantage, a.a.O., S. 62 ff; Cooper, R., Kaplan, R.S., Measure Cost Right: Make the Right Decisions, in: Harvard Business Review, Vol. 66, No. 5, 1988; Horváth, P., Mayer, R., Prozeßkostenrechnung, in: Controlling, Bd. 1, Nr. 4, 1989, S. 214 - 219; Shank, J.K., Strategic Cost Management, New Wine, or just New bottles?, in: Journal of Managemment Accounnting Research, Vol. 1, Fall 1989. Einen guten Überblick gibt Horváth, P., Revolution im Rechnungswesen: Strategisches Kostenmanagement, in: Strategieunterstützung durch das Controlling: Revolution im Rechnungswesen?, (Hrsg.), Horváth, P., Stuttgart, 1990.

3 Als Wertaktivität definiert Porter eine technologisch und strategisch von anderen Aktivitäten unterscheidbare Unternehmensaktivität. Wertaktivitäten lassen sich neun generischen Gruppen zuordnen, diese sind Eingangslogistik, Operationen, Marketing und Vertrieb, Ausgangslogistik, Kundendienst, Unternehmensinfrastruktur, Personalwirtschaft, Technologieentwicklung und Beschaffung. Vgl. Porter, M.E., Competitive Advantage, a.a.O., S. 39, Meffert, H., Funktionenlehre, a.a.O., S. 392. Der in dieser Arbeit geprägte Begriff der Geschäftsaktivität ist mit einer Porterschen Wertaktivität wie folgt zu vergleichen: Eine Geschäftsaktivität kann einer Wertaktivität entsprechen, jedoch auch Teile oder Gruppen von Wertaktivitäten umfassen.

4 Vgl. Porter, M.E., Competitive Advantage, a.a.O., S. 64.

5 Darin ist die strategische Kostenanalyse von der Prozeßkostenrechnung zu unterscheiden. Die Prozeßkostenrechnung (Activity Based Costing) betrachtet zwar wie die strategische Kostenanalyse im wesentlichen die traditionell als indirekt bezeichneten Kosten, jedoch mit anderem Zeithorizont und anderen "zulässigen" Bezugsgrößen. Während die strategische Kostenanalyse die Kostenstruktur sehr grundsätzlich analysiert, legt die Prozeßkostenrechnung die direkten Kosten auf einzelne Tätigkeiten um - analog zur Umlage auf (Fortsetzung der Fußnote auf der nächsten Seite) 
Im zweiten und wichtigsten Schritt der strategischen Kostenanalyse wird für jede einzelne Wertaktivität bestimmt, von welchen Einflußfaktoren ("Cost Drivers") das ermittelte Niveau der Kosten abhängt, ${ }^{1}$ z.B. Skaleneffekte, Lerneffekte, Kapazitätsauslastung, Produktpositionierung, Standorte oder institutionelle Faktoren. Daraus lassen sich Ansatzpunkte zur Verbesserung der Kostenposition ableiten. ${ }^{2}$ Riley unterscheidet zwei Gruppen von Kosteneinflußfaktoren, strukturelle und exekutionelle. Während die strukturellen Kosteneinflußfaktoren die Kostenstruktur bestimmen, beeinflussen die exekutionellen Kosteneinflußfaktoren das Kostenniveau bei gegebener Kostenstruktur. Unter strukturellen Kosteneinflußfaktoren subsumiert Riley Größe, Sortiment, Erfahrung, Technologie und Komplexität. Als exekutionelle Kosteneinflußfaktoren nennt er Kapazitätsnutzung, Produktivität, Produkteffektivität, Qualitätsmanagement, Partizipation und Nutzung der Verbindung zu Lieferanten und Kunden. Diese Kosteneinflußfaktoren sind im Einzelfall zu operationalisieren. ${ }^{3}$

Herstellmengen in der traditionellen Kostenrechnung. Beispielsweise erfaßt die Prozeßkostenrechnung die Kosten pro Angebotserstellung oder pro Forschungsversuch, die strategische Kostenanalyse hingegen betrachtet die Kosten von Angebotserstellung oder Forschung nach Nivau und Einfluß auf die Wettbewerbsposition Zur Prozeßkostenrechnung Vgl. Johnson, H.Th., Activity-Based Information: A Blueprint for World Class Management Accounting, in: Management Accounting, Vol. 69, No. 12, 1988, S. 23 ff; Lee, P.M., The Miccro-Marketing Revolution, in: Small Business Reports, Vol. 15, No. 2, 1990, S. $71 \mathrm{ff}$; Jeans, M., Morrow, M., The Practicalities of Using Activity-Based Costing, in: Management Accounting (UK), Vol. 67, No. 10, 1989, S. 42 - 44; Drury, C., Activity-Based Costing, in: Management Accounting (UK), Vol. 67, No. 8, 1989, S. 60 - 66; Horváth; P., Mayer, R., Prozeßkostenrechnung, a.a.O., S. 214 - 219.

1 Vgl. Porter, M.E., Competitive Advantage, a.a.O., S. 70; Horváth, P., Strategisches Kostenmanagement, a.a.O., S. 182; Shank, J.K., Strategic Cost Management, a.a.O., S. 58. Die Unterscheidung von Kosteneinflußfaktoren geht zurück auf Buaron, R., New-Game Strategies, in: The McKinsey Quarterly, Spring 1981, S. 34 und Bain, J.S., Barriers to New Competition, Cambridge, Massachusetts, 1956, Kapitel 3. In der deutschsprachigen Literatur wird der Begriff "Cost Driver" gelegentlich auch als "Kostentreiber" oder als "kostentreibender Faktor" übersetzt. Vgl. z.B. Horváth, P., Strategisches Kostenmanagement, a.a.O., S. 182; Franz, K.-P., Die Prozeßkostenrechnung im Vergleich mit der flexiblen Plankostenrechnung und der Deckungsbeitragsrechnung, in: Strategieunterstützung durch das Controlling: Revolution im Rechnungswesen?, Hrsg.: Horváth, P., Stuttgart, 1990, S. 197. Dem wird hier die umständlichere, jedoch sinngemäßere Übersetzung in "Kosteneinflußfaktor" vorgezogen.

2 Vgl. Porter, M.E., Competitive Advantage, a.a.O., S. 70 ff. Porters Systematik von Kosteneinflußgrößen erscheint als nicht völlig überschneidungsfrei, deswegen wird die Systematik von Riley herangezogen. Vgl. Horváth, P., Strategisches Kostenmanagement, a.a.O., S. 182 und Shank, J.K., Strategic Cost Management, a.a.O., S. 56 ff .

3 Vgl. Horváth, P., Strategisches Kostenmanagement, a.a.O., S. 182. 
In einem dritten Schritt der strategischen Kostenanalyse ist $\mathrm{zu}$ analysieren, welche Veränderung der Kostenposition aus einer Veränderung der Wertaktivitäten möglich ist. Dazu nennt Porter zwei Hauptansatzpunkte: die Veränderung der Wertaktivitäten entlang der ermittelten Kosteneinflußfaktoren sowie die neue Konfiguration der gesamten Wertkette. ${ }^{1}$ Integrationsentscheidungen können in erheblichem Ausmaß auf beides wirken. So verändert die kontraktuelle Integration eines Wettbewerbers die strukturellen Kosteneinflußfaktoren erheblich, während die technische Integration sowohl die strukturellen (Technologie) als auch die exekutionellen Kosteneinflußfaktoren verändert. ${ }^{2}$ Darüber hinaus kann etwa der Einsatz neuer KIS die Konfiguration der gesamten Wertkette verändern, wie z.B. im Back-Office von Banken geschehen.

\subsubsection{Zeitposition}

Zeit ist in der jüngeren Literatur als dritte Komponente der Wettbewerbsposition einer Unternehmung herausgestellt worden, ${ }^{3}$ und zwar aus zwei Gründen: Erstens ist Zeit für den Kunden ein wichtigeres Qualitätskriterium geworden. Dies verdeutlicht die Tatsache, daß die Zahlungsbereitschaft des Kunden sehr elastisch ist im Bezug auf die Zeit (z.B. Paketzustellung). ${ }^{4}$ Zweitens können mit dieser Maßgröße Erkenntnisse über Prozesse und Abläufe gewonnen werden, die in der kumulierten oder aggregierten

1 Vgl. Porter, M.E., Competitive Advantage, a.a.O., S. 99.

2 Die Operationalisierung der Kosteneinflußfaktoren sowie die Analyse der allgemeinen Wirkungsrichtung von Integrationsentscheidungen erscheint nur für den konkreten Einzelfall sinnvoll. Dies ist aber das Ziel dieses Kapitels B. Erneut sei darauf hingewiesen, da B hier lediglich ein Analyserahmen entwickelt wird, welcher im Kapitel C zur Analyse der wetthewerbsstrategischen Wirkung von Integrationsentscheidungen angewendet wird.

3 Vgl. Stalk, G., Value of Time, a.a.O.; Blackburn, J.D., (Hrsg.), Time-Based Competition, a.a.O.; Stalk, G., Hout, Th.M., Competing Against Time, New York, London, 1990; Stalk, G., Time - The Next Source of Competitive Advantage, in: Harvard Business Review, Vol. 66. No. 4, 1988, S. 41 - 51.

4 Vgl. Stalk, G., Hout, Th.M., Competing Against Time, a.a.O., S.90 ff. 
Bildung von Qualitäts- und Kosteninformationen verloren gehen würden. Damit wird Zeit auch zu einer operativen Steuerungsgröße. ${ }^{1}$

Die Zeitposition der Unternehmung ist wie die Qualitäts- und die Kostenposition aus der Perspektive des Kunden relativ zum Wettbewerb zu definieren. Zur Messung der Komponente Zeit ist dazu ein in sich abgeschlossener, logisch zusammenhängender Zeitraum zu wählen. Dieser wird als "Zyklus" bezeichnet. Vier Zeitzyklen können unterschieden werden: der Produktionszyklus, der Auftragszyklus, der Entwicklungszyklus und der Managementzyklus.

Der Produktionszyklus ist das für die Produktion eines Produkts benötigte Zeitintervall. Der Auftragszyklus ist der Zyklus zwischen Kundenauftrag und Lieferung, er umfaßt damit den Produktionszyklus sowie die Zeit im Distributionssystem. Die isolierte Verbesserung der Zeitposition in der Produktion bleibt in ihrer Wirksamkeit begrenzt, wenn sie nicht auch auf das Distributionssystem ausgedehnt wird. Die Länge des Auftragszyklus hängt vom Integrationsgrad der operativen oder direkten Geschäftsaktivitäten ab. Diese bestimmen die für den Kunden unmittelbar spürbare Zeitposition der Unternehmung.

Von mindestens gleicher Bedeutung ist der Entwicklungszyklus, die Zeit von der Ausarbeitung einer Produktinnovation bis zu deren Implementierung. ${ }^{2}$ Dies einerseits aufgrund von Kundenanforderungen an neue Produkte, andererseits zur zeitlichen Reduktion der erheblichen Kostenvorläufe, die langfristige und unsichere strategische Commitments verursachen. Der Managementzyklus ist die Zeit von der Äußerung eines Kundenproblems im Markt bis zur Umsetzung der Lösung dafür. Der Managementzyklus umfaßt den Entwicklungszyklus sowie zusätzlich die Zeit für

1 Vgl. Stalk, G., Hout, Th.M., Competing Against Time, a.a.O., S. 192. Vgl. die Liste von Turney, P.B.B., Anderson, B., Accounting for Continous Improvemment, in: Sloan Management Review, Vol. 30, No. 2, 1989, S. 45 f.

2 Vgl. z.B. Blackburn, J.D., New Product Development: The New Time Wars, in: TimeBased Competition, (Hrsg.), Blackburn, J.D. a.a.O., S. 127 ff; Stalk, G., Hout, Th.M., (Fortsetzung der Fußnote auf der nächsten Seite) 
Marktforschung und Ideengewinnung einerseits und die Einführung und weitere Implementierung andererseits.

Integrationsentscheidungen wirken offensichtlich in erheblichem Ausmaß auf alle vier Zyklen. Beispielsweise kann durch die Bildung vertikaler Verknüpfungen mit KIS der Produktions- und Auftragszyklus erheblich verkürzt werden, indem unmittelbarer Informationsaustausch und Rückkopplungen möglich werden. Entwicklungs- und Managementzyklus können bedeutend verkürzt werden durch horizontale Verflechtungen mit Koordinationsmechanismen, mit denen Wissen unterschiedlicher Unternehmensteile ausgetauscht wird.

\subsubsection{Dauerhaftigkeit}

Das Hauptproblem bei der Ausschöpfung eines Wettbewerbsvorteils ist dessen Absicherung über den Zeitraum, für den er fixiert wurde. Der Aufbau eines Wettbewerbsvorteils ist nur eine notwendige Bedingung zur Gewährleistung der langfristigen Profitabilität der Unternehmung. Die hinreichende Bedingung ist dessen Dauerhaftigkeit. Diese wird von der Bindungswirkung von Commitments entscheidend beeinflußt. So betont Gälweiler, daß das wohl schwierigste Problem in der Unternehmensplanung in der notwendigen und hinreichenden Einbeziehung der dynamisch verlaufenden Randbedingungen liege, denen die Unternehmensziele von Natur aus ausgesetzt seien - wesentliche Umweltbedingungen änderten sich ständig. ${ }^{1}$ Wie gezeigt, ist diese Anpassung vor allem dann problematisch, wenn (bzw. weil) starre Faktoren, entgangene Optionen und organisationale Trägheit vorliegen. So wurde auch vielfach darauf hingewiesen, daß die Firmen, die gemäß der statischen Erfolgsfaktoren in Peters und Waterman "In Search of Excellence"2 als "erfolgreich" eingestuft wurden,

Competing Against Time, a.a.O., S. $124 \mathrm{f}$.

1 Gälweiler, A., Unternehmensplanung, a.a.O., S. 82.

2 Peters, T., Waterman, J., In Search of Excellence, New York, 1982. 
schon nach wenigen Jahren nicht mehr als erfolgreich gelten können, wenn diese nach denselben Kriterien bewertet werden. ${ }^{1}$

Bei der statischen Betrachtung ${ }^{2}$ von Wettbewerbspositionen wird von der Dynamik des Wettbewerbs, die die Dauerhaftigkeit eines Wettbewerbsvorteils determiniert, abstrahiert. Ein dauerhafter Wettbewerbsvorteil ergibt sich jedoch keineswegs aus einem schlicht großen Vorteil in der Wettbewerbsposition. Das Gegenteil kann der Fall sein. Ein ausgeprägter (profitabler) Wettbewerbsvorteil kann sich in einen hohen Schumpeterschen Pioniergewinn umsetzen, der dann viele Konkurrenten in den Markt lockt, was den Gewinn abschmelzen läßt (vgl. Abb. B.1.8). So läßt eine im Zeitablauf zunehmende Wettbewerbsintensität die Konsumentenrente steigen und die von der Unternehmung erzielbare Rente abnehmen. Um die wettbewerbsstrategische Wirkung von (Integrations)-Entscheidungen mit hoher Bindungswirkung $z u$ analysieren, ist $\mathrm{zu}$ untersuchen, inwieweit sie die Dauerhaftigkeit von Wettbewerbspositionen verändern.

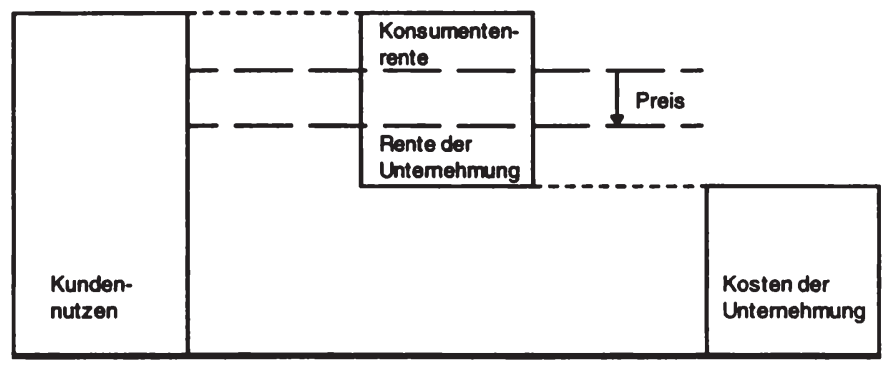

Abb. B.1.8: Konsumenten- und Produzentenrente bei steigender Wettbewerbsintensităt

Die Dauerhaftigkeit einer Wettbewerbsposition ist Ergebnis von drei Faktoren: Stabilität, Flexibilität und Verteilung. Handlungen der Wettbewerber stehen der Stabilität der Wettbewerbsposition gegenüber. Durch Imitation oder durch die Einführung "neuer Regeln" können die Wettbewerber eine

1 Vgl. z.B. Wittek, B., Kundennutzen, a.a.O., S. 67.

2 Bzw. komparativ-statischen Betrachtung (bei Vergleichen von Soll- und Ist-Positionen). 
Erosion der Wettbewerbsposition bewirken. Flexibilität ist in dem Ausmaß gegeben, wie die Unternehmung sich trotz eingegangener Commitments Handlungsoptionen offenhalten kann, die die Irreversibilität von Bindungen begrenzen und damit Unwägbarkeiten in Stabilität und Verteilung begegnen, indem Bindungen gelockert und die Angewiesenheit auf deren Amortisation reduziert werden. Schließlich ist ein Wettbewerbsvorteil vor der Verteilung an Dritte zu sichern, z.B. der Abschöpfung durch monopolistisch agierende Zulieferer.

Stabilität, Flexibilität und Verteilung bestimmen den Zeitraum, über den eine Unternehmung einen geschaffenen Qualitäts-, Kosten- oder Zeitvorteil nutzen und somit Rente realisieren kann.

\subsubsection{Stabilität}

Die Stabilität einer Wettbewerbsposition kann entweder durch Imitation von Konkurrenten oder Einführung neuer Regeln des Wettbewerbs gefährdet werden. Dabei zielt eine Imitationsstrategie darauf ab, durch Produkt- oder Prozeßimitation an der Abschöpfung von Renten in einem Markt zu partizipieren. Sollte den Wettbewerbern die Imitation nicht erfolgversprechend erscheinen, können sie versuchen, neue Wettbewerbsregeln einzuführen, ${ }^{1}$ indem sie eine ähnliche Lösung des Kundenproblems mit einem völlig anders strukturierten Faktorbündel herstellen bzw. das Kundenproblem auf eine andere Weise lösen, als es zuvor der Fall war. In diesem Fall wird das Commitment der Unternehmung von den Konkurrenten nicht kopiert, sondern untergraben.

1 Vgl. Buaron, R., New-Game Strategies, a.a.O., S. 24. 


\subsection{Abwehr von Imitation durch Mobilitätsbarrieren}

Erfolgreiche Unternehmensführung, manifestiert durch hohe Renten, bietet aktuellen und potentiellen Wettbewerbern Anreiz zur Imitation. Imitation läßt die Wettbewerbsintensität steigen und das Erfolgspotential der Unternehmung abnehmen. Mobilitätsbarrieren sichern die Rente aus eingegangen Commitments, indem sie die Imitation der Wettbewerbsposition durch die Konkurrenten erschweren. Unternehmen mit ähnlichen Faktorbündeln werden als strategische Gruppe bezeichnet.

\begin{tabular}{|l|l|l|l|l|}
\hline $\begin{array}{l}\text { Cha- Art } \\
\text { rakter }\end{array}$ & GröBe & Zugang & $\begin{array}{l}\text { Defensiv- } \\
\text { asymmetrie }\end{array}$ & $\begin{array}{l}\text { Wettbewerbs- } \\
\text { reaktion }\end{array}$ \\
\hline $\begin{array}{l}\text { Endogene } \\
\text { Mobilităts- } \\
\text { barrieren }\end{array}$ & $\begin{array}{l}\text { Skalen- und } \\
\text { Sortiments- } \\
\text { effekte }\end{array}$ & $\begin{array}{l}\text { Umschalt- } \\
\text { kosten }\end{array}$ & $\begin{array}{l}\text { Zeitver- } \\
\text { zögerungen }\end{array}$ & \\
\hline & $\begin{array}{l}\text { Erfahrungs- } \\
\text { kurveneffekte }\end{array}$ & $\begin{array}{l}\text { Fixierter } \\
\text { Ressourcen- } \\
\text { zugang }\end{array}$ & $\begin{array}{l}\text { Austritts- } \\
\text { barrieren }\end{array}$ \\
$\begin{array}{l}\text { Strukturellen } \\
\text { Mobilităts- } \\
\text { barrieren }\end{array}$ & $\begin{array}{l}\text { Natürliches } \\
\text { Monopol }\end{array}$ & $\begin{array}{l}\text { Staatliche } \\
\text { Regulierung }\end{array}$ & $\begin{array}{l}\text { Strategische } \\
\text { Commitments }\end{array}$ & Neue Regeln \\
\hline
\end{tabular}

Abb. B.1.9: Mobilitătsbarrieren als Quellen der Stabilitāt der Wettbewerbsposition

Eine strategische Gruppe ist insofern durch sie gemeinsam umgebende Mobilitätsbarrieren definiert. ${ }^{1}$ Mobilitätsbarrieren können nach ihrer Art und ihrem Charakter unterschieden werden (vgl. Abb. B.1.9).

Endogene Mobilitätsbarrieren beruhen unmittelbar auf Entscheidungen der Unternehmung selbst (oder einer Gruppe von Unternehmungen). ${ }^{2}$ Strukturelle Mobilitätsbarrieren resultieren aus exogenen, z.B. Entscheidungen des Staates, oder quasi-exogenen Faktoren, insbesondere in der Vergangenheit getroffene, aber irreversible Entscheidungen, die aufgrund ihrer Irre-

1 Vgl. Caves, R.E., Porter, M.E., Entry barriers, a.a.O., S. $249 \mathrm{ff}$.

2 Vgl. auch Gilbert, R.J., Mobility Barriers and the Value of Incumbency, in: Handbook of industrial organization, (Hrsg.), Schmalensee, R., Willig, R., Amsterdam u.a..O., 1989, S. 495. 
versibilität den Entscheidungsraum der Unternehmung verlassen haben und wie die exogenen Faktoren in den Datenkranz gewandert sind. ${ }^{1}$

Größenvorteile stabilisieren die Wettbewerbsposition, da sie nur in Grenzen imitierbar sind. Skaleneffekte (bzw. Scopeeffekte ${ }^{2}$ ) wirken auf die Kostenposition der Unternehmung durch die Umlage fixer, periodischer Kosten auf die Ausbringungsmenge (bzw. die Menge verschiedener Sorten). Je ausgeprägter die Skaleneffekte eines Unternehmens sind, desto unwahrscheinlicher ist ein Neueintritt eines Wettbewerbers in eine strategische Gruppe, d.h. desto stabiler ist die Kosten- bzw. Wettbewerbsposition. ${ }^{3}$ Liegen Erfahrungseffekte vor, kann mit einem Vorsprung in der relevanten Bestandsgröße (z.B. kumulierter Absatz) eine stabile Wettbewerbsposition aufgebaut werden. Sie ist c.p. gegenüber Imitation zum Zeitpunkt $t$, wenigstens bis zum Zeitpunkt $t+\Delta t$ stabil, wobei der Zeitraum $\Delta t[Z E]$ bestimmt ist aus dem Verhältnis der Differenz der Bestandsgröße [ME] zwischen den betrachteten Wettbewerbern, geteilt durch die Stromgröße [ME/ZE]. ${ }^{4}$ Der Extremfall des Größenvorteils ist das natürliche Monopol. Das natürliche Monopol wird üblicherweise über das Vorliegen einer strikt subadditiven

1 Vgl. Schelling, T.C., The Strategy of Conflict, Boston, 1960, S. 24.

2 Vgl. Teece, D.J., Economies of Scope and the Scope of the Enterprise, in: Journal of Economic Behavior and Organization, Vol. 1, 1980, S. 223. Da kein dem Begriff "Scope" entsprechender deutscher Terminus existiert, wird er nicht übersetzt. Eine einfache Definition der Begriffe, die sich ausschließlich auf die Kosten(-position) der Unternehmung bezieht, ist $C\left(X^{1}+X^{2}\right)<C\left(X^{1}\right)+C\left(X^{2}\right)$ für Skaleneffekte, und $C(X+Y)<C(X)+$ $C(Y)$ für Scopeeffekte; mit $C($.$) der Kostenfunktion der Unternehmung, X^{i}$ zwei Mengen des Guts $X, X$ und $Y$ Mengen verschiedener Güter. Eine gute Übersicht über Scopeeffekte geben Goldhar, J.D., Jelinek, M., Plan for Economies of Scope, in: Harvard Business Review, Vol. 61, November - December 1983, S. 141 - 148.

3 Vgl. Ghemawat, P., Sustainable Advantage, in: Harvard Business Review, Vol. 64, 1986, S. 55.

4 Beispiel: A hat 1 Mio. Telefone verkauft, B 2 Mio. A und B sind die einzigen Anbieter auf dem Markt jenes Telefons, auf dem mit der Rate von 0,5 Mio. pro ZE nachgefragt wird. A benötigt c.p. selbst bei Verkaufsstopp von B zumindest $\Delta t=2 \mathrm{ZE}$, um die Wettbewerbsposition von B zu erreichen. Sollte B weiterhin anbieten, aber A zumindest höhere Absatzmengen erzielen, ist die Bestandsgrößendifferenz durch die Stromgrößendifferenz zu dividieren. Gleichwohl ist die Wettbewerbsposition nicht gegen "neue Regeln" stabil, und selbst das bloße Aufrechterhalten von Erfahrungseffekten bedarf ständiger Fortentwicklung. Vgl. dazu Turney, P. B. B., Anderson, B., Continous Improvement, a.a.O., S. 41 ff. 
Kostenfunktion definiert, ${ }^{1}$ womit nur eine Unternehmung eine stabile Wettbewerbsposition aufbauen kann, z.B. Wasserversorgungsunternehmen. Subadditive Kostenfunktionen führen zu extremen Skaleneffekten, die häufig auf exogen gegebenen, schwer veränderlichen Gegebenheiten beruhen. Sie sind aufgrund mangelnder Imitierbarkeit als sehr stabil anzusehen. ${ }^{2}$ Eine besondere Form solcher Skaleneffekte sind Netzwerkeffekte (Network Economies) 3 Sie führen zu Strukturen, die einem natürlichen Monopol nahekommen. So ist es sowohl für die Betreiber wie für die Nutzer von elektronischen Netzwerken vorteilhaft, daß möglichst viele Anbieter/Abnehmer angeschlossen sind, beispielsweise bei einem Telefon- oder einem geschlossenen Informationsnetz.

Unabhängig von Größeneffekten kann eine Wettbewerbsposition durch eine nicht kopierbare Sicherung des Zugangs zu Kunden, Lieferanten oder Produktionsfaktoren stabilisiert werden (vgl. Abb. B.1.9). Eine endogene Zugangsbarriere ist die Schaffung von Umschaltkosten (switching costs). Dies sind dem Kunden entstehende Kosten des Wechsels zu einem Konkurrenten. ${ }^{4}$ Beispiele sind die Lernkosten beim Wechsel des Textverarbeitungsprogramms oder der Aufbau eines neuen Vertrauensverhältnisses, etwa beim Wechsel des Zahnarztes. Umschaltkosten werden erhöht, wenn KIS eingerichtet werden, z.B. Bestell- oder Liefersysteme zwischen Produzent, (Groß-) Händler und Abnehmer. Nimmt beispielsweise der Abnehmer seine gesamte Lagerhaltung und Bestellung über das von einem Händler gestellte System vor, ${ }^{5}$ verbessert sich nicht nur seine Abwicklungseffi-

1 Eine Kostenfunktion $C(y)$ ist strikt subadditiv bei der Ausbringungsmenge y, wenn für alle Ausbringungsvektoren $y^{i}$ mit $\sum_{i} y^{i}=y$ gilt, daß $C(y)<\sum_{i} C\left(y^{i}\right)$. . Vgl. Panzar, J.C., Determinants of Firm and Industry Structure, in: Industrial Organization, (Hrsg.), Schmalensee, R., Willig, R., a.a.O., 1989, S. 23.

2 Panzar zeigt jedoch, daß Skaleneffekte allein weder notwendig noch hinreichend für das Vorliegen eines natürlichen Monopols sind und daß Skalen- und Scopeeffekte zusammen zwar notwendige, aber nicht an jedem Punkt hinreichende Bedingung für das Vorliegen eines natürlichen Monopols sind. Vgl. Panzar, J.C. Determinants of Firm, a.a.O., S.24 ff.

3 Vgl. Gilbert, R.J., Mobility Barriers, a.a.O., S. 506 ff.

4 Gilbert, R.J., Mobility Barriers, a.a.O., S. 506 ff.

5 Dies ist auch ein gutes Beispiel für qualitative Scopeeffekte: Nur ein Händler, der (fast) den gesamten Bedarf des Abnehmers decken kann, wird eine derart weitgehende Inte(Fortsetzung der Fußnote auf der nächsten Seite) 
zienz, sondern es werden auch enge Bindungen zwischen allen Beteiligten aufgebaut. Ein Wechsel auf ein anderes System wird dabei um so teurer, je enger sich Produzent, Händler und Abnehmer technisch und rechtlich an das System binden. Auch vertragliche Regelungen können den Zugang zu Kunden, Lieferanten oder Produktionsfaktoren fixieren und direkte Mobilitätsbarrieren begründen, die kurzfristig weder imitiert noch umgangen werden können. Ein solcher fixierter Zugang zu Ressourcen bedeutet quasi prohibitiv hohe Umschaltkosten und begründet ein "Lock-in" der (umfassend verstandenen) Ressource ${ }^{1}$ (z.B. Produktionstechniken, Managementtechniken, Zugang zu Märkten bzw. zum Handelskanal). Aus einer exogenen Zugangsbarriere, der staatlichen Regulierung, kann eine Wettbewerbsposition hoher Stabilität resultieren, bei der Imitation schwer vorstellbar ist. Allerdings ist Wettbewerb auf Basis neuer Regeln denkbar, wie etwa durch Einrichtung privater Funktelefonnetze und Telefaxdienste als Konkurrenz zum öffentlichen Telefon- und Briefdienst.

Unter dem Begriff Defensivasymmetrie sind Stabilität begründende Faktoren zusammengefaßt, die auf der bloßen Existenz einer Wettbewerbsposition beruhen und sich nicht aus den Mobilitätsbarrieren Größe oder Zugang ergeben (vgl. Abb. B.1.9). Sie bestehen gegenüber potentiellen Wettbewerbern, die (noch) keine vergleichbare Wettbewerbsposition aufgebaut haben. Zeitverzögerungen (Response Lags) liegen vor, wenn eine Wettbewerbsposition auf komplexen technologischen oder organisationalen Prozessen beruht, deren Imitation nicht beliebig beschleunigt werden kann. ${ }^{2}$ Zeitverzögerungen entstehen durch die Zeitspanne zwischen der Beobachtung von Signalen im Markt und a) deren Umsetzung in sinnvolle Erkenntnisse, b) der Formulierung von und Entscheidung über Reaktionsmaßnahmen, c) deren Implementierung und d) ihrer Wirkung im Markt. Aus-

grationspolitik mit dem Abnehmer durchführen können.

1 Vgl. Farrell, J., Shapiro, C., Dynamic Competition with Lock-in, Working paper, Department of Economics, University of California at Berkely, 1986, S. 1 ff; vgl. Bain, J.S., Industrial Organization, 2nd ed., New York, 1968, S. 261. Bain unterscheidet solche Effekte als "absolute Kostenvorteile" von den Skaleneffekten, welche von der Produktionsmenge abhängen.

2 Vgl. Ghemawat, P., Sustainable Advantage, a.a.O., S. 57 f. 
trittsbarrieren sind Kosten oder entgangene Gewinne, die einer Unternehmung entstehen, wenn sie aus einem Markt ${ }^{1}$ austritt. ${ }^{2}$ Die Höhe von Austrittsbarrieren hängt ab a) von der Art des gebundenen Produktivvermögens, b) den Fixkosten des Marktaustritts, c) den Verbundwirkungen, d) den emotionalen und sozialen Vorbehalten und (e) dem Verhalten des Staates bzw. der Gewerkschaften. ${ }^{3}$ Marktaustrittsbarrieren wirken direkt und indirekt auf die Defensivasymmetrie. Einerseits senken Marktaustrittsbarrieren bereits die Mobilität direkt durch den Erwartungswert der Austrittskosten zum Eintrittszeitpunkt, d.h. des einzugehenden Wagnisses für den Fall, daß sich die angestrebte Wettbewerbsposition nicht aufbauen läßt und ein Austritt bzw. erneute Mobilität erforderlich ist. Indirekte Wirkungen ergeben sich aus der höheren Wettbewerbsintensität bei hohen Austrittsbarrieren, insbesondere bei Überkapazitäten. ${ }^{4}$ Marktaustrittsbarrieren sind partiell endogen, partiell strukturell: Sie ergeben sich einerseits aus dem (beeinflußbaren und imitierbaren) Wesen des Marktes, der Produkte und der Technologie. Andererseits beruhen etwa die Fixkosten des Marktaustritts auf dem Zusammenwirken mehrerer Unternehmungen einer strategischen Gruppe, zudem ist das Verhalten Dritter (Arbeitnehmer, Staat usw.) eher als exogen zu charakterisieren.

Als strategische Commitments seien diejenigen Commitments bezeichnet, die bewußt zum Aufbau struktureller Mobilitätsbarrieren eingegangen werden. Sie sind der einzige Weg, strukturelle Mobilitätsbarrieren selbst aufzubauen, andere strukturelle Mobilitätsbarrieren (natürliches Monopol, staatlich regulierter Zugang) beruhen demgegenüber auf den Gegebenheiten oder Handlungen Dritter. Mit strategischen Commitments kann die

1 Die für den Marktaustritt vorgetragene Argumentation kann auf den Austritt aus einer strategischen Gruppe übertragen werden.

2 Vgl. Gilbert, R.J., Mobility Barriers, a.a.O., S. 520.

3 Meffert, H., Ohlson, G., Was Sie beim Marktein- und -austritt beachten müssen, in: Die Absatzwirtschaft 1982, Nr. 10, Sonderausgabe, 1982, S. 178 - 190; vgl. auch Meffert, H., Katz, R., Unternehmensverhalten in stagnierenden und gesättigten Märkten: Ergebnisse einer empirischen Untersuchung in der Bundesrepublik Deutschland, Wissenschaftliche Gesellschaft für Marketing und Unternehmensführung, Münster, 1983.

4 Vgl. Dixits Modell zur Abschreckung des Markteintritts (Entry Deterrence). Dixit, A., The Role of Investment in Entry Deterrence, Economic Journal, Vol. 90, 1981; Gilbert, R.J., (Fortsetzung der Fußnote auf der nächsten Seite) 
Unternehmung jedoch quasi-exogene Gegebenheiten selbst herstellen. Eine Bindung an die Wettbewerbsposition kann eine Unternehmung beispielsweise durch den Aufbau von anderweitig nicht einsetzbaren, also starren (Über)kapazitäten erreichen. Mit der Investition in starre Faktoren signalisiert die Unternehmung gegenüber aktuellen und potentiellen Wettbewerbern den Willen zur künftigen Nutzung dieser Faktoren, zur Verteidigung der Wettbewerbsposition. Erhebliche bereits eingegangene Kostenvorläufe mögen die Wettbewerber vom Markteintritt abhalten, d.h. ihrer Mobilität begrenzen. Die Logik strategischer Commitments ist dabei das "Versenken" von Kapital in dauerhafte, starre und knappe Faktoren. Die Faktoren müssen dauerhafter Natur sein, also Potentialfaktoren wie Anlagen, Humankapital und Brand Equity, weil sie sonst nicht Stabilität im Zeitablauf begründen können. Sie müssen starr sein, weil sonst Kapital nicht wirklich versinkt, sondern eine jederzeitige Veräußerung der Faktoren möglich wäre zu marktgängigen Opportunitätskosten, die den Ausgangsaufwendungen entsprechen. ${ }^{1}$ Ohne die Starrheit entsteht keine Bindung. Die Faktoren müssen knapp sein, weil sich sonst aus ihrem Einsatz keine Rente erzielen läßt. Gelingt es einer Unternehmung, strategische Commitments in starre, knappe, dauerhafte Faktoren einzugehen, kann sie die strukturelle Stabilität ihrer Wettbewerbsposition erreichen, d.h. ihre Wettbewerber in eine Situation versetzen, die die Imitation der Wettbewerbsposition ökonomisch nachteilig werden läßt.

\subsection{Neue Regeln zur Umgehung von Mobilitätsbarrieren}

Während endogene Mobilitätsbarrieren prinzipiell überwindbar sind, können strukturelle Mobilitätsbarrieren nur durch "neue Regeln" umgangen werden $^{2}$, da Imitationsstrategien entweder nicht möglich oder ökonomisch nicht vorteilhaft sind. Dabei werden die Gegebenheiten in einem Markt

Mobility Barriers, a.a.O., S. 520.

1 Vgl. Gilbert, R.J., Mobility Barriers, a.a.O., S. $521 \mathrm{ff}$.

2 Buaron, R., New-Game Strategies, in: The McKinsey Quarterly, Spring 1981, S. 27ff. 
nicht als unveränderbar hingenommen, sondern neu definiert in enger Abstimmung mit dem partiell fixen Faktorbündel (= den Stärken). Die Neudefinition kann sich dabei wie auch die Imitation auf den Gesamtmarkt oder auf einzelne Segmente beziehen. Eine verbreitete Taxonomie der Optionen ist in Abb. B.1.10 angegeben.

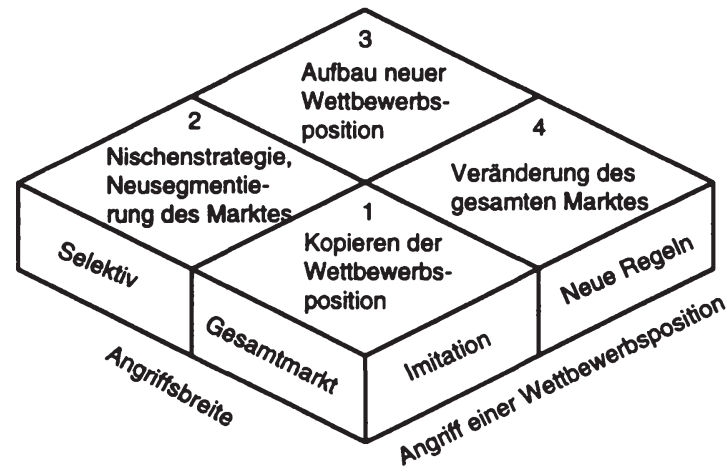

Abb. B.1.10: Strategic Gameboard

Quelle: Buaron, New Game Strategies, a.a.O., S. 27

Neue Regeln der Wettbewerber können eine Wettbewerbsposition untergraben, die durch strukturelle Mobilitätsbarrieren gesichert ist. Die Stabilität einer Wettbewerbsposition sicherzustellen bedeutet daher auch, mögliche neue Regeln zu antizipieren, insbesondere falls erhebliche strategische Commitments eingegangen werden (müssen). Die Antizipation ist jedoch besonders schwierig, weil die Strategie "neuer Regeln" auch kleineren Konkurrenten oder Konkurrenten aus anderen Branchen möglich ist. Typische Situationen für "neue Regeln" sind1:

(1) Neue Produktionstechnologien

(2) Hohe Wettbewerbsintensität auf Basis alter, eingefahrener Regeln

(3) Veränderungen in der sozialen und rechtlichen Umwelt, die die Wettbewerber verschieden stark beeinflussen

1 Buaron, R., New-Game Strategies, a.a.O., S. 36 f. 
(4) Substitutionsprodukte, die den Kundennutzen oder die Kundenkosten erheblich verändern

(5) Veränderung der Inputfaktoren

(6) Übercommitment des Marktführers, das ihn unbeweglich macht

(7) Neue vertikale Strukturen in der Branche (rechtliche Vorwärtsoder Rückwärtsintegration oder Deintegration)

(9) Änderungen der Regulierung oder des Wettbewerbs unter Lieferanten oder Abnehmern

(10) Statisches Wettbewerbsgleichgewicht in der Branche und Verhalten nach identischen Regeln.

Deshalb sollte zunächst eine Situationsanalyse durchgeführt werden, und zwar unabhängig von der aktuellen Definition des Marktes, der Wettbewerbskräfte, der Art der aktuellen Lösung des Kundenproblems oder der Prozeß- bzw. Produkttechnologien. Ziel ist es, mögliche Faktorbündel für drohende "Neue Regel-Strategien" zu lokalisieren.

Die Verteidigung gegen neue Regeln ist jedoch problematisch. Die alten Regeln bestimmen sowohl die Wettbewerbsposition als auch deren Stabilität, mithin das Ausmaß und die Dauer der erzielbaren Rente. Ein Gegenangriff auf die im Aufbau befindliche Wettbewerbsposition eines Wettbewerbers, der nach anderen Regeln operiert, ist nicht immer möglich, da ein Wechsel zu den neuen Regeln den Wert der alten Regeln und damit die erzielbare Rente gefährdet.

Die Schaffung neuer Regeln des Wettbewerbs zieht allerdings ihrerseits das Eingehen von Commitments nach sich. Buaron gibt das Ausmaß einzugehender Commitments von den in Abb. B.1.10 genannten Optionen als steigend von Quadrant 1 nach 4 an. Dies ergibt sich aus dem Charakter des Faktorbündels, das die Commitments ausmacht: Je stärker und breiter eine angestrebte Regelveränderung ist, desto ausgeprägter muß eine Investition in starre Faktoren sein. Daraus folgen höhere Opportunitätskosten für entgangene Optionen und eine sinkende Reversibilität angesichts organisationaler Trägheit. Dabei ist ein sequentielles Vorgehen durch verschiedene Quadranten möglich. ${ }^{1}$

1 Von besonderer Bedeutung ist dabei die Frage des Timings der einzelnen Schritte. Vgl. (Fortsetzung der Fußnote auf der nächsten Seite) 


\subsubsection{Flexibilität}

Eine Wettbewerbsposition ist flexibler als eine andere, wenn sie ein größeres Set von künftigen Optionen ermöglicht. Wettbewerbspositionen beruhen auf Commitments in partiell fixe Faktoren. Dabei sind nicht vollständig reversible Entscheidungen zu treffen. Im Nachhinein kann sich das Eingehen eines Commitments als nachteilig herausstellen, und zwar als derart nachteilig, daß trotz partieller Irreversibilität die Strategie gewechselt wird.

Dabei ist "Flexibilität (...) keine Strategie per se; sie ist eine Eigenschaftsdimension von Strategien". ${ }^{1}$ Mithin kann eine Wettbewerbsposition als mehr oder weniger flexibel bezeichnet werden. Die auf zukünftigen Handlungsoptionen beruhende Flexibilität, die Handlungsflexibilität, ist von der Built-in-Flexibilität zu unterscheiden. Die Built-in-Flexibilität zielt auf die Sicherung der Unternehmung durch Streuung der Geschäftsaktivitäten, so $\mathrm{da}$ der Erfolg der Unternehmung resistent ist gegenüber unsicheren Umwelteinflüssen. ${ }^{2}$ Für die weitere Diskussion ist die Handlungsflexibilität näher zu betrachten. ${ }^{3}$ Innerhalb der Handlungsflexibilität unterscheidet Meffert die Aktionsflexibilität als Menge der Handlungsspielräume, die Prozeßflexibilität als Handlungsschnelligkeit und die Strukturflexibilität als Handlungsbereitschaft. 4

Damit ist die Flexibilität einer Wettbewerbsposition der Umkehrfall der Bindung an sie, der Umkehrfall eines Commitments. Unternehmensflexi-

Buaron, R., New-Game Strategies, a.a.O., S. 31 ff; Remmerbach, K.-U., Markteintrittsentscheidungen, Wiesbaden, 1988, besonders S. $39 \mathrm{ff}$.

1 Meffert, H., Größere Flexibilität als Unternehmenskonzept, a.a.O., S. 123.

2 Vgl. Meffert, H., Größere Flexibilität als Unternehmenskonzept, a.a.O., S. 124.

3 Zur Kritik der Konzeption der Built-in-Flexibilität und der zugrundeliegenden Diversifikationsstrategien vgl. Meffert, H., Größere Flexibilität als Unternehmenskonzept, a.a.O., S. $124 \mathrm{f}$.

4 Meffert, H., Größere Flexibilität als Unternehmenskonzept, a.a.O., S. 126. 
bilität ist dabei kein Selbstzweck, sondern nur dann von Wert, wenn die zugrundeliegende Wettbewerbsposition einen positiven (Erwartungs-)Wert hat, ${ }^{1}$ (ebenso wie eine stabile Wettbewerbsposition und eine günstige Verteilung der Rente). Im folgenden soll am Beispiel der Einführung eines Expertensystems ${ }^{2}$ aufgezeigt werden, inwieweit sich durch Aktionsflexibilität ein zusätzliches Erfolgspotential aus einer flexiblen Wettbewerbsposition versus einer nicht flexiblen Wettbewerbsposition ergibt.

Eine Unternehmung diskutiert eine Modifikation ihrer Wettbewerbsposition durch die Umstellung zentraler Geschäftstätigkeiten auf ein großes Expertensystem. So hat beispielsweise das Kreditkartenunternehmen American Express die Entscheidungen über die Anfragen von Vertragshändlern, ob die Belastung eines Kartenkunden akzeptiert wird, auf ein Expertensystem umgestellt. Diese Umstellung betrifft sehr entscheidende Geschäftsaktivitäten von American Express. Einerseits ist das Unternehmen auf die Zuverlässigkeit der Abwicklung angewiesen, ohne die ein erhebliches Risiko aus überzogenen Kreditlinien bestünde. Zudem ist die Reibungslosigkeit und Geschwindigkeit der Zusage ein wichtiges Kriterium für den Kartenkunden bei der Auswahl der Karte am Point of Sale. Für ein Projekt der Einführung eines derart bedeutenden Expertensystems steht die Unternehmung vor der Frage, wieviel Flexibilität vorgesehen werden und wann das Projekt möglicherweise abgebrochen werden soll. Das Vorsehen von Flexibilität bringt zwar Flexibilitätsvorteile mit sich, mag aber u.a. Kostennachteile nach sich ziehen $^{3}$.

1 Vgl. Meffert, H., Zum Problem der betriebswirtschaftlichen Flexibilität, in: Zeitschrift für Betriebswirtschaft, 39. Jg. 1969, Heft Nr. 12, S. 798; Meffert, H., Die Flexibilität in betriebswirtschaftlichen Entscheidungen, Habilitationsschrift (eingereicht bei der Staatswirtschaftlichen Fakultät der Ludwig-Maximilians-Universität München), München, 1968, S. 7.

2 Zum Begriff des Expertensystems vgl. Kap. 2.3.1.1.4

3 Vgl. Meffert, H., Größere Flexibilität als Unternehmenskonzept, a.a.O., S. 123. 


\subsection{Erwartungswert der Aktionsflexibilität}

Betrachtet wird ein Totalzeitraum T, der in zwei Perioden unterteilt ist: Periode 1 bis zum Zeitpunkt $\tau=t$, innerhalb der der Prototyp des Expertensystems entwickelt wird, und danach die Periode 2, innerhalb der das Expertensystem weiterentwickelt und eingesetzt wird. Vereinfachend wird die zukünftige Unsicherheit dichotom abgebildet, d.h. entweder als Erfolg $S$ oder Mißerfolg F.

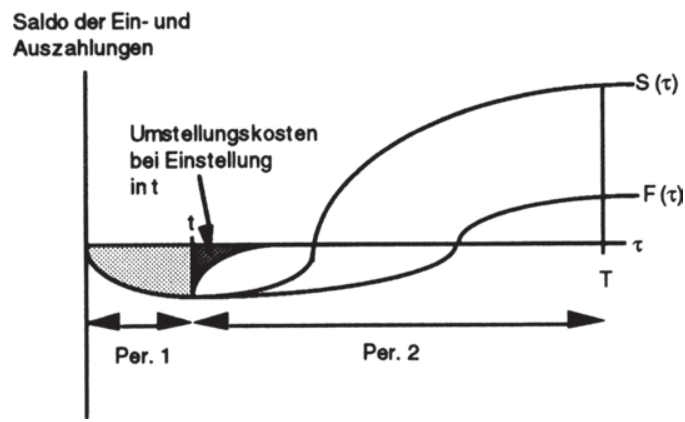

Abb. B.1.11: Schematische Darstellung der Zahlungsprofile eines erfolgreichen (S) und eines nicht erfolgreichen (F) Szenarios

Es seien $C(\tau)$ die Momentanauszahlungen der Erstentwicklung des Expertensystems zum Zeitpunkt $\tau, S(\tau)$ die Momentaneinzahlungen zum Zeitpunkt $\tau$ aus dem Einsatz des Expertensystems im Erfolgsfall und $F(\tau)$ die Momentaneinzahlungen aus dem Betrieb des Expertensystems in $\tau$ im Mißerfolgsfall. (vgl. Abb. B.1.11)

Es sei DC der Barwert der Momentanauszahlungen, abgezinst auf den Zeitpunkt $\tau=0$, analog DF (DS) der Barwert im Erfolgsfall (Mißerfolgsfall), abgezinst auf den Zeitpunkt $\tau=0 .{ }^{1} \mathrm{Um}$ einen Erwartungswert für den Zah-

1 Ausführliche Schreibweise des Barwerts vgl. Abschnitt 1.1.1. 
lungsüberschuß zu ermitteln, sei angenommen, daß aus Erfahrungen mit ähnlichen Projekten in der Vergangenheit a priori Wahrscheinlichkeiten gewonnen werden können. ${ }^{1}$ Es sei bekannt, daß das Expertensystem erfolgreich eingesetzt werden kann $(S)$ mit einer Wahrscheinlichkeit $p(S)=0,6$. Damit ist $p(F)=0,4$.

Der erwartete Barwert E1 des Expertensystems ergibt sich als:

$$
E 1=D C+p(S) D S+p(F) D F=D C+0,6 D S+0,4 D S .
$$

Beim Barwert E1 ist Flexibilität nicht vorgesehen. Eine genauere Analyse möge ergeben, daß die a priori angenommene Wahrscheinlichkeit $p(S)=0,6$ eine vereinfachende Aggregation darstellt, die in zwei Fälle zu unterscheiden ist. Das Expertensystem kann in seiner technischen Funktion entweder gut oder fehlerdurchsetzt sein. Der erste Zustand sei als "Hit", der zweite als "Bug" bezeichnet. Es wird nun a priori angenommen, daß die Erfolgswahrscheinlichkeit davon abhängt, ob das Expertensystem zum Zeitpunkt $t$ bereits gut oder fehlerdurchsetzt ist, und zwar mit folgenden bedingten Wahrscheinlichkeiten:

$$
\begin{array}{ll}
\mathrm{P}(\mathrm{S} \mid \mathrm{HIT})=0,9, & \mathrm{p}(\mathrm{F} \mid \mathrm{HIT})=0,1 \\
\mathrm{p}(\mathrm{S} \mid \mathrm{BUG})=0,3, & \mathrm{p}(\mathrm{F} \mid \mathrm{BUG})=0,7 .
\end{array}
$$

A priori ist jedoch unbekannt, ob das Expertensystem am Startzeitpunkt sich als Hit oder als Bug herausstellen wird. Es wird angenommen daß:

1 Für Entscheidungsprobleme unter Unsicherheit werden in der Literatur alternativ Entscheidungsregeln vorgeschlagen, die die Gewinnung solcher a priori Wahrscheinlichkeiten verzichtbar machen (z.B. die Minimax-Regel, die Maximin-Regel, die HurwiczRegel oder die Annahme gleicher Wahrscheinlichkeiten für jeden der möglichen Umweltzustände). Als Zwischenlösung werden Verfahren zur Gewinnung von a prioriWahrscheinlichkeiten aus Vergangenheitsdaten oder Experimenten vorgeschlagen. Ziel der nachstehenden Analyse ist es nicht, diese Verfahren und deren Vorteilhaftigkeit ou diskutieren, sondern die Erwartungswerte des Vorsehens und Vernachlässigens von Flexibilität zu darzustellen. Zur Bildung der Erwartungswerte werden aus Vergangenheitswerten oder Experimenten abgeleitete a priori Wahrscheinlichkeiten angenommen. Vgl. als Übersicht über die Vor- und Nachteile der Verfahren Luce, R.D., Raiffa, H., Games and Decisions, Paperback Reprint 1989, New York, 1957, S. 13 f., S. 275 ff., S. 309 ff. 
(4) $\quad \mathrm{p}(\mathrm{HIT})=\mathrm{p}(\mathrm{BUG})=0,5$.

Damit ergibt sich wieder:

(5) $\quad \mathrm{p}(\mathrm{S})=\mathrm{p}(\mathrm{S} \mid \mathrm{HIT}) \mathrm{p}(\mathrm{HIT})+\mathrm{p}(\mathrm{S} \mid \mathrm{BUG}) \mathrm{p}(\mathrm{BUG})=0,6$ sowie

(6) $p(F)=0,4$

Nun kann am Ende der ersten Periode beobachtet werden, ob sich der Prototyp des Expertensystems als erfolgreich oder nicht erfolgreich herausgestellt hat. Mit dieser Information kann die Einschätzung der künftigen Situation aktualisiert werden. Aus den Erfahrungen der ersten Periode kann eine verbesserte Prognose darüber abgegeben werden, ob sich das System in dem tatsächlichen Einsatz als Hit oder Bug herausstellen wird. Formal ist diese Aktualisierung die Bildung von A-posteriori-Wahrscheinlichkeiten aus den A-priori-Wahrscheinlichkeiten auf Basis des Bayeschen Theorems. ${ }^{1}$

In der ersten Periode ist beobachtet worden, daß das Expertensystem als Erfolg einzustufen ist. Damit ist:

$$
p(\text { HITIS })=\frac{p(s \mid H I T) p(H I T)}{p(S)}=\frac{0,90,5}{0,6}=0,75
$$

(8) $\quad \mathrm{p}(\mathrm{BUG})=\frac{\mathrm{p}(\mathrm{s} \text { BUG }) \mathrm{p}(\mathrm{BUG})}{\mathrm{p}(\mathrm{S})}=\frac{0,30,5}{0,6}=0,25$

Unter der Bedingung, daß in der ersten Periode $\mathrm{S}$ eintrat, hat sich a posteriori die Wahrscheinlichkeit eines erfolgreichen Einsatzes geändert zu:

$$
\begin{aligned}
\mathrm{p}(\mathrm{S}) & =\mathrm{p}(\mathrm{S} \mid \mathrm{HIT}) \mathrm{p}(\mathrm{HIT} \mid \mathrm{S})+\mathrm{p}(\mathrm{S} \mid \mathrm{BUG}) \mathrm{p}(\mathrm{BUG} \mid \mathrm{S}) \\
& =0,75
\end{aligned}
$$

1 Zum Bayeschen Theorem vgl. z.B. Luce, R.D., Raiffa, H., Games and Decisions, a.a.O., S. 312; Hax, H., Laux, H., Flexible Planung, a.a.O., S. 321. 
(10)

$$
\begin{aligned}
p(F) & =p(F \mid H I T) p(H I T \mid S)+p(F \mid B U G) p(B U G \mid S) \\
& =0,25
\end{aligned}
$$

Analog ist $\mathrm{p}(\mathrm{HIT} \mid \mathrm{F})=0,125$ und $\mathrm{p}(\mathrm{BUG} \mid \mathrm{F})=0,875$ und unter der Bedingung, daß in der ersten Periode $F$ eingetreten ist, $p(S)=0,375$ und $p(F)=$ 0,625 .

Der Barwert aus dem Expertensystem wurde in (3) bezeichnet mit $D C+0,6$ DS + 0,4 DS. Dieser Barwert entspricht der nicht flexiblen Vorgehensweise: Es wird a priori entschieden, ob das Expertensystem entwickelt und eingesetzt wird oder nicht. Bei Einsatz wird der Barwert des Erfolgsfalls und des Mißerfolgsfalls mit den jeweiligen Eintrittswahrscheinlichkeiten multipliziert. Auf Basis der nunmehr ermittelten A-posteriori-Wahrscheinlichkeiten kann der nicht flexible Barwert auch aufgespalten werden in:

$$
\begin{aligned}
\mathrm{E} 1= & \mathrm{DC}+0,6(0,75 \mathrm{DS}+0,25 \mathrm{DF}) \\
& +0,4(0,375 \mathrm{DS}+0,625 \mathrm{DF})^{1} .
\end{aligned}
$$

Im Vergleich dazu ist der Barwert der flexiblen Vorgehensweise

$$
\text { (11) } \mathrm{E} 2=\mathrm{DC}+0,6(0,75 \mathrm{DS}+0,25 \mathrm{DF})+0,4 \mathrm{DU} \text {, }
$$

mit DU als den auf den Zeitpunkt $\mathrm{t}=0$ diskontierten Umstellungskosten. Dem liegt folgende Überlegung zugrunde. Sollte sich in der Periode 1 das Expertensystem als erfolgreich herausstellen, erhöht sich die Erfolgswahrscheinlichkeit auch für Periode 2 - das Projekt wird fortgesetzt. Stellt sich aber ein Mißerfolg heraus, wird das Projekt abgebrochen, allerdings nur dann, wenn ein Abbruch vorteilhafter ist als die Weiterführung, also wenn ein "Abbruchgewinn" oder "Umstellungsgewinn" vorliegt. Dieser Umstellungsgewinn setzt sich zusammen aus den verhinderten Verlusten DF, den durch Abbruch entgangenen, aber noch möglich gewesenen Gewinnen DS

1 Ausmultiplikation ergibt wieder DC + 0,6 DS + 0,4 DF. 
sowie den Umstellungskosten. Im Beispiel ist der Erwartungswert des Umstellungsgewinns im Zeitpunkt $\tau=t$ wenn zuvor $F$ beobachtet wurde:

$$
\begin{aligned}
\text { Umstellungsgewinn } & =\mathrm{DU}-\mathrm{p}(\mathrm{F}) \mathrm{p}(\mathrm{S}) \mathrm{DS}-\mathrm{p}(\mathrm{F}) \mathrm{DF} \\
& =\mathrm{DU}-0,375 \mathrm{DS}-0,625 \mathrm{DF}
\end{aligned}
$$

Ein Abbruch erfolgt, wenn der Umstellungsgewinn positiv ist, anders ausgedrückt, wenn der erwartete negative Cash Flow aus der Weiterführung kleiner ist als die Umstellungskosten. Der Wert der Flexibilität im Zeitpunkt $t=0$ ergibt sich aus der Differenz der erwarteten Barwerte E1 und E2 in $t=0$, also der Differenz des Barwerts mit Flexibilität und ohne Flexibilität als:

$$
\text { (13) } \begin{aligned}
\mathrm{VF} & =\mathrm{E} 2-\mathrm{E} 1 \\
& =\mathrm{p}(\mathrm{F})[\mathrm{DU}-\mathrm{p}(\mathrm{S}) \mathrm{DS}-\mathrm{p}(\mathrm{F}) \mathrm{DF}] \\
& =0,4[\mathrm{DU}-0,375 \mathrm{DS}-0,625 \mathrm{DF}] \\
& =\quad 0,4 \mathrm{DU}-0,15 \mathrm{DS}-0,25 \mathrm{DF}
\end{aligned}
$$

mit

$\mathrm{p}(\mathrm{F}) \mathrm{DU}=0,4 \mathrm{DU} \quad$ Erwartungswert der Umstellungskosten. Da der Term DU negativ ist, senkt dieser den Umstellungsgewinn. Die Umstellungskosten ergeben sich aus den starren Faktoren, aus der Bindungswirkung von Commitments.

$\mathrm{p}(\mathrm{F})[-\mathrm{p}(\mathrm{S}) \mathrm{DS}] \quad=0,15 \mathrm{DS}$ : Erwartungswert der entgangenen Gewinne aufgrund der Einstellung des Expertensystems nach dem Ende der ersten Periode, obwohl mit dieser Wahrscheinlichkeit doch noch der Erfolgsfall eintritt. Da der Term DS positiv ist, senken die entgangenen Gewinne den Umstellungsgewinn. Wie die Umstellungskosten ergeben sich die entgangenen Gewinne aus der Bindungswirkung von Commitments, sie mindern die Erzielung von Renten, wie eingangs verbal beschrieben. 
$\mathrm{p}(\mathrm{F})[-\mathrm{p}(\mathrm{F}) \mathrm{DF}] \quad=0,25 \mathrm{DF}$ : Erwartungswert der vermiedenen Verluste durch die Einstellung des Expertensystems nach dem Ende der ersten Periode. Der Term DF ist negativ, ${ }^{1}$ die Verlustvermeidung bei erkannten "Bugs" ist der Grund, warum ein Umstellungsgewinn überhaupt existiert. Der Erwartungswert vermiedener Verluste ist also die Basis eines Umstellungsgewinns und damit die Basis des Werts der Flexibilität.

Aus (12) und (13) ist ersichtlich, daß der Wert der Flexibilität allgemein angegeben werden kann als

(14) $\mathrm{VF}=\mathrm{p}(\mathrm{F})$ Umstellungsgewinn.

Allgemein ergibt sich der Wert, Flexibilität vorzusehen, aus der a priori erwarteten Wahrscheinlichkeit, umstellen zu müssen, multipliziert mit dem Umstellungsgewinn. Der Umstellungsgewinn muß annahmegemäß größer Null sein, sonst würde eine Umstellung unterlassen. Der Umstellungsgewinn ist positiv, wenn die erwarteten Umstellungskosten und die erwarteten entgangenen Gewinne kleiner sind als die erwarteten vermiedenen Verluste, folglich wenn sich aus der Auflösung der Bindungswirkung von Commitments ein positiver Erwartungswert ergibt. Damit beruht der Wert der Flexibilität auf der Auflösung der Bindungswirkung von Commitments.

Das Vorsehen von Flexibilität kann somit den Wert einer Wettbewerbsposition erhöhen. Folglich kann es sinnvoll sein, z.B. Stabilitätsvorteile zugunsten von Flexibilitätsvorteilen aufzugeben. Zwar ermöglicht das Eingehen strategischer Commitments, also das bewußte Ausschließen von Flexibilität, eine Absicherung gegen Imitation. Besteht jedoch zwischen Flexibilität und Stabilität einer Wettbewerbsposition ein konkurrierendes, substitutives Verhältnis, sind die Vor- und Nachteile der Bindungswirkung strategischer Commitments abzuwägen. Dabei sollte in Bezug auf die Dimension "Stabilität" eine hohe Bindungswirkung, in Bezug auf die Dimension

1 Sonst würde das Expertensystems nicht eingestellt. 
"Flexibilität" eine niedrige Bindungswirkung der die Wettbewerbsposition begründenden Commitments angestrebt werden. Dies ist die Logik, die den KIS-gestützten flexiblen Fertigungssystemen zugrunde liegt. Diese haben im Hinblick auf die potentielle Produktionskapazität einer Branche eine hohe Bindungswirkung, da flexible Fertigungsssysteme hohe versunkene Kosten aufweisen, sind sie einmal beschafft. Somit kann eintretenden Unternehmen glaubwürdig angedroht werden, auf lange Sicht zu extrem niedrigen Grenzkosten anzubieten. Damit kann die Wettbewerbsposition erheblich stabilisiert werden. Im Hinblick auf die Dimension Flexibilität erlauben flexible Fertigungssysteme jedoch eine erheblich Variantenvielfalt und inkrementale Änderungen im Zeitablauf. Damit werden neben der Bindungswirkung zur Stabilisierung der Wettbewerbsposition Freiheitsgrade zur Flexibilisierung der Wettbewerbsposition geschaffen - und beide tragen zur dauerhaften Erzielung von Rente bei.

\subsection{Einflußfaktoren auf den Erwartungswert der Flexibilität}

Aus der dargestellten Ermittlung des Erwartungswerts lassen sich die folgenden vier Faktoren ableiten, die den (Erwartungs-)Wert der Flexibilität bestimmen:

(1) Umstellungswahrscheinlichkeit. Die A-priori-Wahrscheinlichkeit, umstellen zu müssen, [hier: $p(F)$ ] geht direkt in den Wert der Flexibilität ein. Dies erscheint auch plausibel. Denn je geringer die Wahrscheinlichkeit einer Umstellung ist, desto weniger ist Flexibilität im Ausgangszeitpunkt anzustreben.

(2) Umstellungskosten. Eingegangene Commitments verursachen Bindungen und damit Umstellungskosten und entgangene Optionen. Die Umstellungskosten und die Opportunitätskosten entgangener Optionen gehen, multipliziert mit der Wahrscheinlichkeit, umstellen zu müssen, in den Wert der Flexibilität ein. Je höher diese Kosten für das Schwenken zwi- 
schen Alternativen, d.h. je ausgeprägter Commitments sind, desto weniger wird Flexibilität wert sein.

(3) Wertdifferenz strategischer Optionen. Je weniger vorteilhaft am Ende von Periode 1 die Weiterführung ist und je vorteilhafter die Alternative, desto höher wird dies auf den Wert der Flexibilität durchschlagen. Im Beispiel sind dies die Alternative "Null" und die Weiterführung mit dem Erwartungswert, der aus DS und DF gebildet wird. Je näher nun dieser Erwartungswert an Null liegt, desto geringer ist der Wert der Flexibilität, umgekehrt je höher die vermiedenen Verluste, desto größer ist der Wert der Flexibilität.

(4) Lerngeschwindigkeit. Lernen, aufgefaßt als Abnahme von Unsicherheit, begründet den Wert der Flexibilität überhaupt. Lernen wäre ausgeschlossen, wenn sich die A-priori-Wahrscheinlichkeit und die A-posteriori-Wahrscheinlichkeit gleichen würden. Lernen findet im Beispiel allerdings statt. Die A-priori-Wahrscheinlichkeit des Erfolgs, $p(S)=0,6$, wird je nach Beobachtung der ersten Periode a posteriori entweder auf $p(S)=0,75$ oder $P(S)=$ 0,375 verändert. Erst mit dieser Veränderung ergibt sich die Möglichkeit, die Alternativen neu zu bewerten.

In Abb. B.1.12 werden drei Fälle unterschieden: der im Text diskutierte Fall 1, der Fall 2, in dem kein Lernen vorkommt, und der Fall 3, in dem maximal mögliches Lernen in Periode 3 stattfindet. "Kein Lernen" wird abgebildet durch eine identische Wahrscheinlichkeit des Erfolgs oder Mißerfolgs für die Fälle, daß das Expertensystem ein Hit oder ein Bug ist.

Damit kann zum Zeitpunkt $\tau=t$ keine bessere Prognose abgegeben werden, als dies zum Zeitpunkt $\tau=0$ der Fall war. Somit wird sich keine Umstellung ergeben. Damit wird der Erwartungswert mit Flexibilität E2 identisch mit E1. Folglich ergibt sich als Differenz aus beiden der Wert der Flexibilität zum Zeitpunkt $\tau=0$ als Null. Demgegenüber fände vollständiges Lernen statt, würde aus der Beobachtung der ersten Periode mit vollständiger 
Sicherheit auf die Zukunft geschlossen werden können. Zum Ende der ersten Periode wird damit bekannt, ob in der zweiten Periode das Szenario S oder das Szenario F eintritt.

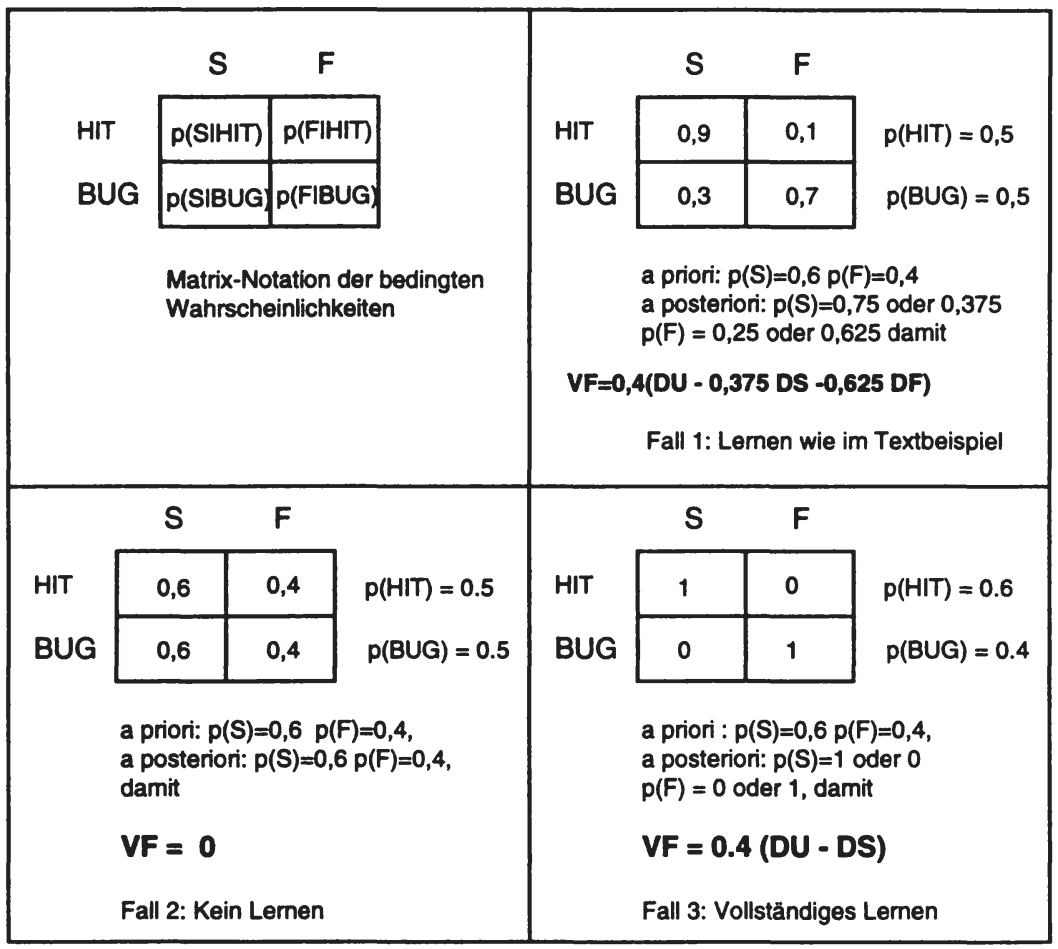

Abb. B.1.12: Lemen und Wert der Flexibilităt

Somit wird am Ende der ersten Periode entweder das Expertensystem eingeführt und erfolgreich betrieben oder ein Abbruch vorgenommen. Damit ist der Wert der Flexibilität maximal: E1 $=0,6 \mathrm{DF}+0,4 \mathrm{DS}$ und E2 $=0,6 \mathrm{DF}+0,4$ $\mathrm{DU}, \mathrm{VF}=\mathrm{E} 2$ - E1 = 0,4(DU - DS). VF wird nicht durch den Term DF geschmälert, während DS voll zu Buche schlägt: Sämtliche möglichen Verluste DS könnten vermieden werden, keine Gewinne DF würden entgehen. 
Lernen, d.h. die Abnahme von Unsicherheit, ist damit von entscheidender Bedeutung für den Wert der Flexibilität. Für Integrationsentscheidungen können aus diesen Überlegungen Ansatzpunkte zur Lerngeschwindigkeit, zur Definition von erklärenden Zuständen im Lernprozeß und zum inkrementalen Lernen gewonnen werden.

Je höher die Lerngeschwindigkeit desto früher sind Anpassungen möglich. Angesichts der Bindungswirkung von Commitments können dadurch sowohl Umstellungskosten $p(F)$ DU und entgangene Gewinne $p(F)$ DS gesenkt als auch Verluste $p(F)$ DF vermieden werden. Die Geschwindigkeit des Lernens hängt dabei von der Zeit für die nötige Beobachtung und von der Spreizung der bedingten Wahrscheinlichkeiten ab. Die Zeit für die nötige Beobachtung im Beispiel ist die Zeit t; wird sie verkürzt, steigt die Lerngeschwindigkeit. Die Lerngeschwindigkeit entspricht der im Zusammenhang mit Commitments diskutierten begrenzten Wandlungsfähigkeit von Organisationen wie $p(F)$ DU den spezifischen Faktoren und $p(F)$ DS den entgangen Gewinnen.

Von erheblicher Bedeutung ist, wie die erklärenden Zustände im Lernpro$z e ß$ definiert werden, und zwar im Hinblick auf erstens die Spreizung und zweitens die Beobachtbarkeit. Im Beispiel wurden beobachtete Zwischenresultate (S und F) auf erklärende Zustände (Hit und Bug) zurückgeführt und daraus künftige Resultate abgeleitet. Je stärker nun die Spreizung der bedingten Wahrscheinlichkeit ist, desto höher ist der Wert der Flexibilität. Deshalb sind erklärende Zustände so zu definieren, daß sie eine besonders große Spreizung der bedingten Wahrscheinlichkeiten erlauben. ${ }^{1}$ Zudem ist zu beachten, daß schnell aus Beobachtungen auf die Zustände geschlossen werden kann - andernfalls wird der Zeitpunkt t so spät liegen, daß der Wert der Flexibilität durch die Verzögerung aufgezehrt wird.

1 Dabei besteht das Risiko der nicht beobachteten Heterogenität (unobserved heterogeneity), das auch als Simpsons Paradoxon in der Literatur diskutiert wird. Vgl. Cohen, J.E., An Uncertainty Principle in Demography and the Unisex Issue, in: The American Statistician, Vol. 40, February 1986, S. 35 f. 
Eine weiterer Ansatzpunkt ist das mehrperiodige Lernen. Durch die Wiederholung von Lernprozessen ergibt sich ein kumulativer Effekt. Im Beispiel könnte die Periode 1 auch in zwei oder mehrere Teilperioden unterteilt werden, in denen jeweils $\mathrm{S}$ versus $\mathrm{F}$ beurteilt wird. Diese "inkrementale Lernstrategie" von häufigen, kleinen Veränderungen kann in vielen Märkten (Fotoapparate, Computer, zunehmend auch Automobile) beobachtet werden. Die Logik kleiner Schritte besteht im häufigen Einholen von Feedback-Informationen aus dem Markt, die dann zu größeren Lerneffekten kumuliert werden können, als dies im Einperiodenfall möglich ist. Durch inkrementales Lernen erhöht sich die Spreizung der bedingten Wahrscheinlichkeiten schneller, da sich die Erkenntnisse aus mehreren Perioden multiplikativ kumulieren. Zudem erhöht sich der Wert der Flexibilität aus zusätzlichen Handlungsoptionen. Verluste $p(F)$ DF können schneller (früher) vermieden werden. Außerdem haben kleine Schritte den Vorteil, daß sie nur geringe Umstellungskosten DU verursachen - sie erhöhen damit den Wert der Flexibilität auf beiden Seiten der Gleichung. ${ }^{1}$

Für die Beurteilung der Wirkung strategischer (Integrations)-Entscheidungen konnten damit vier Faktoren gewonnen werden, die den Wert der Flexibilität einer Wettbewerbsposition bestimmen. Das Vorsehen von Flexibilität trägt c.p. um so stärker zur Erzielung von Rente bei, desto höher die Umstellungswahrscheinlichkeit ist, desto niedriger die Umstellungskosten sind, desto größer die Wertdifferenz der gegebenen strategischen Optionen ist sowie desto höher die Lerngeschwindigkeit ist.

1 Ein Nachteil der inkrementalen Lernstrategie ist die häufigere Bekanntgabe von Informationen an die Konkurrenten, die dann leichter imitieren können. Damit besteht erneut ein Konflikt zwischen Flexibilität und Stabilität der Wettbewerbsposition, allerdings nur dann, wenn die Konkurrenten nicht ohnehin über die Informationen verfügen, die sie durch eine inkrementale Lernstrategie im Vergleich zu einer "revolutionären" Lernstrategie erhalten. 


\subsubsection{Verteilung}

Die Existenz einer (stabilen) Rente besagt noch nichts über deren Verteilung. Die Rente, die aufgrund eines von einer Unternehmung aufgebauten) Wettbewerbsvorteils erzielt wird, fließt nicht notwendigerweise den Eigentümern der Unternehmung in voller Höhe zu, sondern kann im Zeitablauf an andere Stakeholder abfließen, und zwar entweder durch Abschöpfung oder durch Nichtausnutzung (Slack). (vgl. Abb. B.1.13) Stakeholder sind dabei Dritte, die die Macht haben, an der Rente einer Unternehmung zu partizipieren (z.B. Absatzkanal, Staat, Arbeitnehmer).

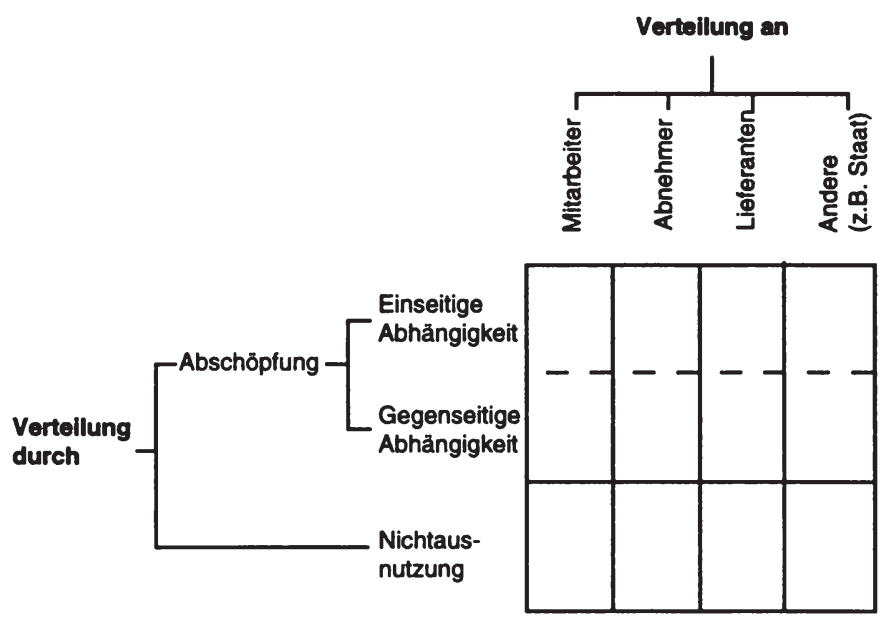

Abb. B.1.13: Systematik des Verteilungsproblems

\subsection{Abschöpfung}

Das partiell fixe Faktorbündel, das die Voraussetzung zur Erzielung von Rente bildet, steht in der Regel nicht vollständig unter der Kontrolle der Kapitaleigner der Unternehmung, sondern wird von verschiedenen Stakeholdern gestellt. Können die dazu beitragenden Faktoren nicht in die Unternehmung internalisiert werden, (z.B. Humankapital, Zulieferer, Ab- 
nehmer), entsteht insbesondere bei einer hohen Rente das Risiko der Abschöpfung. ${ }^{1}$

Die Höhe des Abschöpfungsrisikos wird bedingt von der Art der Abhängigkeit der Parteien voneinander. Beispielsweise kann der Wettbewerbsvorteil eines Reisebüros für "Last-Minute"-Angebote auf dem Zugang zu einem elektronischen Markt für Reisedienstleistungen basieren, verbunden mit der Ausrichtung seines eigenen Geschäftssystems auf diesen elektronischen Markt, z.B. durch langjährigen Schulung der Mitarbeiter, der Anpassung des eigenen Abwicklungssystems, dem Standort an einem Flughafen oder mit einer kostenorientierten Markt- und Marketingstrategie. Die Rente aus dem Vorteil des Reisebüros ist vom Lieferanten des Reisebüros, dem Betreiber des elektronischen Marktes, prinzipiell abschöpfbar. Dies gilt, solange das Abhängigkeitsverhältnis einseitig ist. Denn der Betreiber des elektronischen Marktes kann viele andere Reisebüros bedienen, das Reisebüro jedoch nicht auf alternative elektronische Märkte ausweichen. Ist jedoch das Abhängigkeitsverhältnis zweiseitig, wird auch die Abschöpfung begrenzt. Beispielsweise ist der Reiseanbieter auf seine speziell geschulten Mitarbeiter angewiesen, jene aber auch auf die Anstellung bei diesem Reiseanbieter, weil hier ihre Arbeitsleistung eine höhere (Grenz-)Produktivität erbringt als anderswo. Die Möglichkeiten der Absicherung vor solchen asymmetrischen Abhängigkeiten sind entweder externe Kontrollinstanzen (einklagbare Verträge) oder selbst durch das Herstellen einer gegenseitigen Abhängigkeit der Vertragspartner. ${ }^{2}$

Damit ist die Analyse der Verteilung von Rente zwischen einer Unternehmung und dem sie umgebenden Geschäftssystem bei Integrationsentscheidungen von entscheidendem Stellenwert. Durch Integrationsentscheidungen, z. B. die elektronische vertikale Verknüpfung von Zulieferern, Unternehmung und Absatzkanal mittels KIS, werden die technischen, kontraktuellen und organisationalen Beziehungen im Geschäftssystem völ-

1 Vgl. z.B. Klein, B., Crawford,R., Alchian, A.A., Vertical Integration, a.a.O., S. 297 ff.

2 Vgl. dazu das Geisel-Modell von Williamson, der auch eine Systematik der Absicherungsformen für asymmetrische Abhängigkeitsverhältnisse gibt. Williamson, (Fortsetzung der Fußnote auf der nächsten Seite) 
lig neu geregelt. Durch die Verbindung kann aus einer wettbewerblichen n:m:o-Beziehung eine 1:m:1-Beziehung wird, ${ }^{1}$ insoweit von der Unternehmung ein Commitment in eine Integration eingegangen werden, die sie der quasi-monopolistischen Abschöpfung durch Zulieferer und/oder Absatzkanal aussetzt.

\subsection{Nichtausnutzung}

Erzielte Renten aus dem Einsatz von Produktionsfaktoren bedeuten die Realisierung eines Potentials. Die Nichtausnutzung (Slack) kann dabei verstanden werden als der Teil des Erfolgspotentials der Unternehmung, der nicht in Erfolg (Renten) umgesetzt wird, sondern nur durch zusätzliche Maßnahmen nutzbar wäre: "The crucial, but plausible, assumption here is that there is some 'slack' in the economy; and that additional investment, hours of work, productivity, and decision making can be squeezed out of it by pressure mechanisms."2 Die Nichtausnutzung von Erfolgspotential kann auf eine Reihe zusammenhängender, keineswegs überschneidungsfreier Gründe zurückgeführt werden. Diese sind erstens Unsicherheit über die Produktionsfunktion und die Produktionsfaktoren, zweitens Zielkonflikte zwischen Stakeholdern sowie drittens mangelnder Wettbewerbsdruck.

Leibenstein sieht in der Unsicherheit die Erklärung für suboptimalen Erfolg, d.h. für die Nichtausnutzung von Erfolgspotential. ${ }^{3}$ Aufgrund von

O.E., Economic Institutions, a.a.O., S. 79, S. 85 ff.

1 Dabei sei $\mathrm{n}$ eine beliebig hohe Zahl von Zulieferern, $\mathrm{m}$ die Zahl der Konkurrenten, einschließlich der betrachteten Unternehmung mit ihren Konkurrenten, sowie o die Zahl der Absatzkanäle.

2 Hirshman, A.O., Economic Development, Research and Policy Making: Some Converging Views, in: Behavioral Science, Vol. 7, April 1962, S. 211 f; vgl. Cyert, R.M., March, J.G., Behavioral Theory, a.a.O., S. 36.

3 Leibenstein, H., Allocative Efficiency versus X-Efficiency, in: American Economic Review, Vol. 56, June 1966, S. 398 ff. Empirische Belege finden sich bei Leibenstein, H., Beyond Economic Man, Cambridge, Massachusetts, 1976, S. 34 - 44. Die Nichtausnutzung (Slack) nennt Leibenstein X-Ineffizienz und definiert sie als Abweichung vom theoretischen Optimalzustand der vollständigen allokativen Effizienz. 
Unsicherheit, z.B. über das vielfältige Zusammenwirken der Produktionsfaktoren, die Verfügbarkeit der Faktoren und ihre Beschaffenheit werden optimale Zustände verfehlt. Deswegen wird aufgrund von Unsicherheit immer ein Teil des eigentlich vorhandenen Erfolgspotentials nicht genutzt. 1

Cyert und March weisen auf Zielkonflikte als Ursache für die Nichtausnutzung von Erfolgspotentialen hin. ${ }^{2}$ Die Maximierung von Renten aus dem Faktorbündel der Unternehmung ist Ziel der Eigentümer. Dem können Individual- oder Gruppenziele anderer Stakeholder entgegenstehen. Beispielsweise kann das Management mehr an der Maximierung von Größe und Umsatz als an der Maximierung von Erfolgspotential und Unternehmenswert interessiert sein. ${ }^{3}$ Der Ausgleich von Zielkonflikten wird von Cyert und March als Verhandlungsprozeß einer (instabilen) Koalition portraitiert. Ein solcher Verhandlungsprozeß hat nicht nur distributive Inhalte, sondern auch kooperative. ${ }^{4}$ Distributive Zielkonflikte ziehen in aller Regel auch eine Reduktion des kooperativ erzielbaren Werts nach sich. Auch March und Simon argumentieren, daß (anderweitig) unvereinbare Zielkonflikte in Unternehmungen durch Nichtausnutzung von Erfolgspotential abgemildert würden. 5

Mangelnder Wettbewerbsdruck mindert den Zwang zu Effizienz und Innovation; umgekehrt trägt hoher Wettbewerbsdruck zur Sicherung des Überlebens einer Unternehmung bei. Damit kann die Nichtausnutzung von Erfolgspotential durch (Wettbewerbs-)Druck vermindert werden. ${ }^{6}$ Dies kann

1 Auch Galbraith betont den Zusammenhang von Unsicherheit und Nichtausschöpfung von Erfolgspotential (Slack). Vgl. Galbraith, J.R., Complex Organizations, a.a.O., S. 22 ff.

2 Cyert, R.M., March, J.G., Organizational Factors in the Theory of Oligopoly, in: Quarterly Journal of Economics, Vol. 70, 1956, S. 44 - 64.

3 Vgl. Williamson, O.E., The Economics of Discretionary Behavior: Managerial Objectives in the Theory of the Firm, Englewood Cliffs, New Jersey, 1964, S. $38 \mathrm{ff}$.

4 Formal wird häufig zwischen Nullsummenspielen und Nicht-Nullsummenspielen unterschieden, in der Theorie der Verhandlung oft zwischen "Value Creating" und "Value Claiming" im "Negotiator's Dilemma".Vgl. Lax, D.A., Sebenius, J.K., The Manager as Negotiator, New York, London, 1986, S. 1 ff.

5 March, J.G., Simon, H.A., Organizations, a.a.O., S. 126.

6 Hirshman, A.O., Economic Development, a.a.O., S. 211 f. 
mit dem Konzept des Satisficing vom March und Simon erklärt werden. ${ }^{1}$ Angesichts begrenzter Rationalität wählen Entscheidungsträger "befriedigende" (satisfactory) Lösungen aus ermittelten Alternativen. Bei schwachem Wettbewerbsdruck wird die Zahl der ermittelten Alternativen und das Niveau einer "befriedigenden" Lösung gering ausfallen, da keine "herausfordernden Maßstäbe" zu höheren Niveaus Anreiz geben.2 Die Erreichung dauerhafter Wettbewerbsvorteile zielt in ihrer Logik auf eine Reduktion des Wettbewerbsdrucks. Durch sinkenden Wettbewerbsdruck sind die geschaffenen Erfolgspotentiale der Gefahr der Nichtausnutzung ausgesetzt, dies um so mehr, desto ausgeprägter und dauerhafter ein Wettbewerbsvorteil wahrgenommen wird. Demgegenüber reduziert höherer Wettbewerbsdruck die Nichtausnutzung. Neben höheren "Satisficing"-Anspruchsniveaus reduziert eine hohe Wettbewerbsintensität auch die Unsicherheit. Die Existenz guter Wettbewerber ermöglicht die Übernahme und Fortentwicklung von Know-how, d.h. ergänzt Lernen durch Erfahrung u m Lernen durch Beobachtung. Zudem zwingt Wettbewerbsdruck, die Zielkonflikte der Stakeholder der Unternehmung in einer Weise zu lösen, die das Erfolgspotential möglichst gut ausnutzt. ${ }^{3}$

Integrationsentscheidungen können in erheblichem Ausmaß auf die (Nicht-) Ausnutzung wirken. So können KIS eingesetzt werden, die Informationsbasis von Entscheidungsträgern $z u$ verbessern und damit deren Unsicherheit $\mathrm{zu}$ reduzieren. Mit Koordinationsinstrumenten kann angestrebt werden, Zielkonflikte und daraus folgende Nichtausnutzung zu begrenzen. Zudem beschränkt eine Wettbewerbsdynamik, die durch Einsatz neuer Integrationsinstrumente, insbesondere KIS, verursacht wird, die Nichtausnutzung.

1 Vgl. March, J.G., Simon, H.A., Organizations, a.a.O., S. 140 f.

2 Frank, R.H., Choosing the Right Pond, Human Behavior and the Quest for Status, New York, 1985, S. 3 ff. Er zeigt die fundamentale Bedeutung von wettbewerblichen "Benchmarks" für menschliches Verhalten auf.

3 Vgl. dazu March, J.G., Simon, H.A., Organizations, a.a.O., S. 126. 


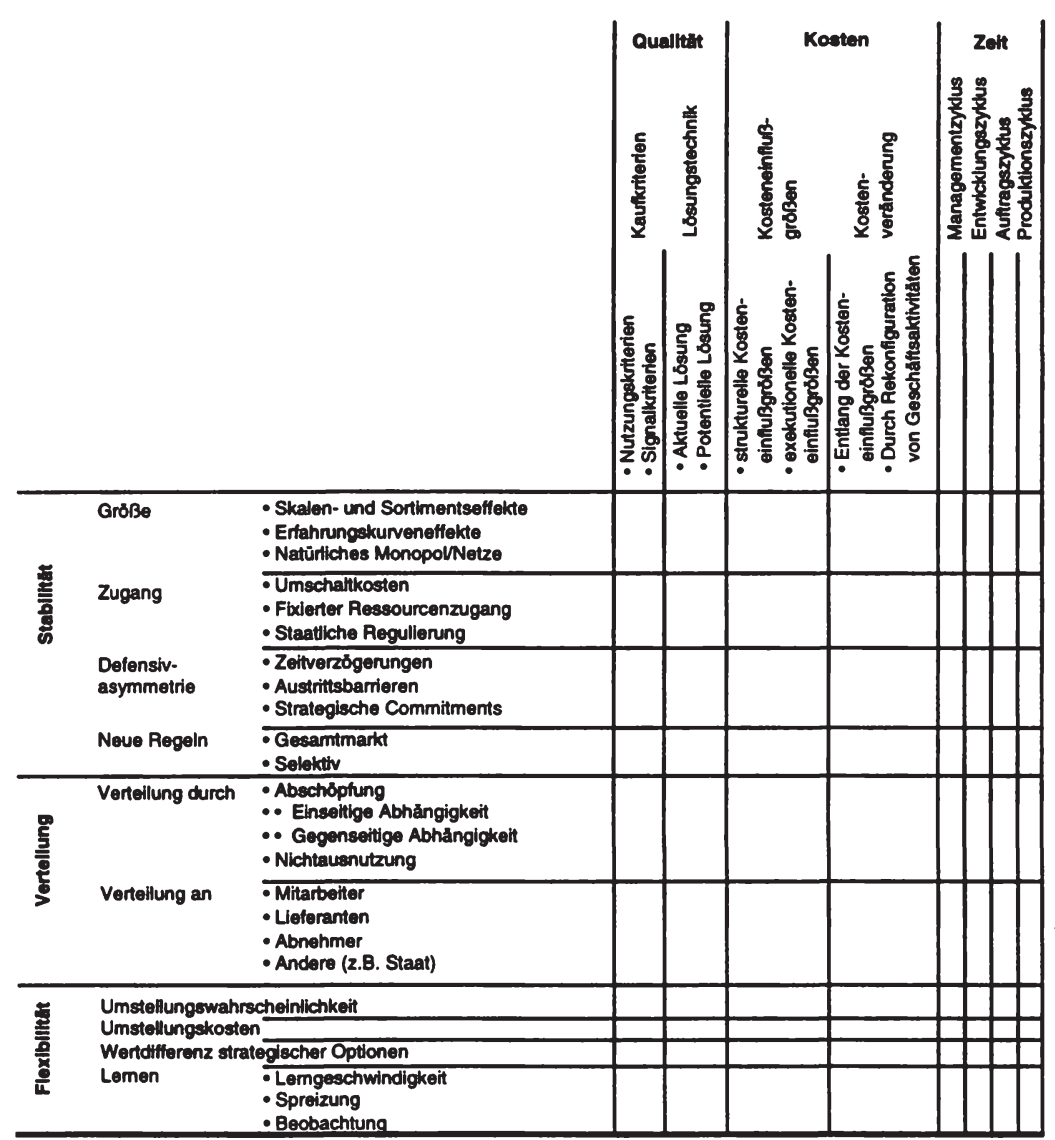

Abb. B.1.14: Ubersicht über die Einflußfaktoren aut den dauerhaften Wettbewerbsvorteil

Als Zusammenfassung des Kapitels B.1.2 sind die diskutierten EinflußgröBen auf den dauerhaften Wettbewerbsvorteil in Abb. B.1.14 dargestellt, analog zur Abbildung B.1.6.

Integrationsentscheidungen haben erhebliche Auswirkungen auf den dauerhaften Wettbewerbsvorteil, und zwar sowohl auf die Wettbewerbsposition als auch auf deren Dauerhaftigkeit. Es liegt nun ein Gerüst vor, mit dem die Wirkung von Integrationsentscheidungen auf den dauerhaften Wettbewerbsvorteil im einzelnen analysiert werden kann. Aufgrund der 
Vielfältigkeit der Wirkungszusammenhänge ist es jedoch nicht möglich, bereits an dieser Stelle eine allgemeine Beurteilung der strategischen Wirkung der Integration von Geschäftsaktivitäten vorzunehmen, also die Frage zu beantworten, unter welchen allgemeinen Bedingungen Integration unter besonderer Berücksichtigung der KIS zum dauerhaften Wettbewerbsvorteil beiträgt oder nicht. Voraussetzung dazu ist, den Möglichkeitsraum von Integrationsentscheidungen genauer $\mathrm{zu}$ bestimmen und verschiedene Integrationsstrategien $\mathrm{zu}$ unterscheiden. Dazu sind die weiteren Strukturelemente von Integrationsentscheidungen zu konkretisieren. 
Das zeitablaufbezogene wettbewerbsstrategische Grundmodell des gebundenen Wettbewerbs ermöglicht die Beurteilung einer gegebenen Integrationsstrategie im Hinblick auf die Zielgröße des dauerhaften Wettbewerbsvorteils. Neben dem Ziel sind für die Entwicklung und die Entscheidung über eine Integrationsstrategie die weiteren Strukturelemente der Integrationsentscheidung zu bestimmen. Dies umfaßt die Definition des Integrationsbereichs und die Wahl der Integrationsinstrumente in Abhängigkeit von den im Integrationsbereich gegebenen situativen Bedingungen. Für die Entscheidung über die so konkretisierte Integrationsstrategie müssen schließlich ihr Nutzen, also ihr Beitrag zum dauerhaften Wettbewerbsvorteil, und ihre Kosten bestimmt werden. Der mit diesen Strukturelementen aufgespannte Möglichkeitsraum von Integrationsstrategien wird im folgenden entwickelt.

\subsection{Integrationsbereiche als Gegenstand der Integration}

Integration ist auf ein konkretes Objekt zu beziehen, nämlich auf einen Integrationsbereich (vgl. Abb. B.2.1). Dabei ist anzugeben, zwischen welchen Elementen (Unternehmensteilen) welche Verbindungen (vertikale Verknüpfungen, horizontale Verflechtungen) betrachtet werden sollen. Integrationsbereiche sind nicht gegeben. Vielmehr sind sie durch die Identifikation von Schnittstellen und Gleichartigkeiten zwischen Geschäftsaktivitäten überhaupt zu definieren, bevor eine Analyse erfolgen kann, durch welche integrativen Verbindungen Schnittstellen abgebaut und Gleichartigkeiten ausgenutzt werden können. ${ }^{1}$ Dieses Problem der Definition zusammenhängender, interdependenter Bereiche ähnelt dem Problem der

1 Schnittstellen und Gleichartigkeiten zwischen Geschäftsaktivitäten weisen auf Integrationsbedarfe aufgrund vorliegender Interdependenzen hin. Dies wird im Abschnitt B.2.1.2 genauer erläutert. 
Marktsegmentierung: Bevor der Einsatz von Marketinginstrumenten sinnvoll analysierbar ist, sind intern homogene und extern heterogene Kundengruppen $z u$ definieren. ${ }^{1}$ Wie die Bestimmung des Integrationsbereichs handelt es sich dabei um ein Problem, daß nur teilweise analytischen Kategorien zugänglich ist und überwiegend durch einen kreativen Prozeß gelöst werden muß. Als Technik zur

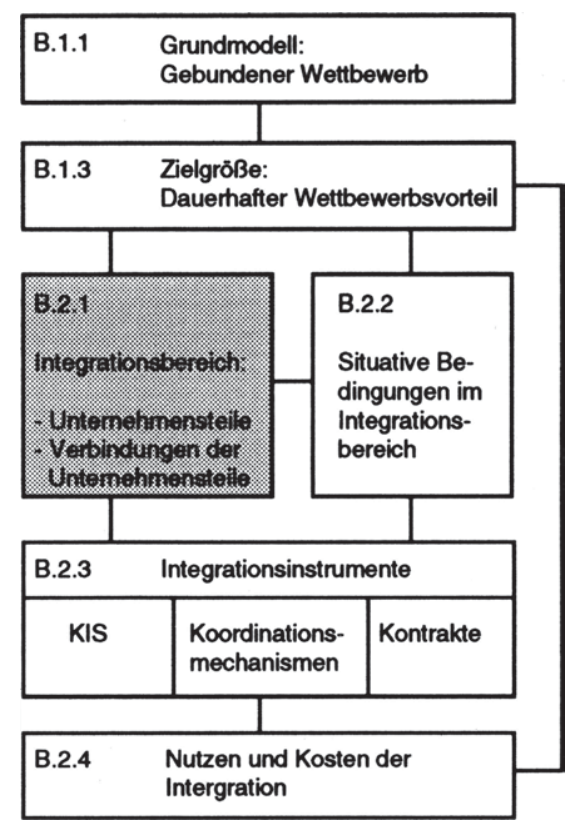

Abb. B.2.1: Stellung des Integrationsbereichs im Bezugsrahmen

Definition von Integrationsbereichen wird das "Mapping" vorgeschlagen. Dieses wird im folgenden beschrieben. Daran schließt sich die Analyse integrativer Verbindungen an.

1 Vgl. Meffert, H., Marketing, Grundlagen der Absatzpolitik, 7. Aufl., Wiesbaden, 1987 S. 243-245. 


\subsubsection{Mapping zur Bestimmung des Integrationsbereichs}

Das Mapping stammt aus der verhaltenswissenschaftlich orientierten Organisationstheorie und wird insbesondere in der Organisationspsychologie eingesetzt. Eine Map enthält danach die wesentlichen Variablen von Vorgängen in einem System, einschließlich der Beziehungen der Variablen zueinander. Die Map wird von Personen konstruiert, die wiederholt Vorgänge in dem System beobachten konnten. ${ }^{1}$ Dabei erfolgt eine subjektive Konzeptionalisierung kognitiver interpersonaler Wirkungszusammenhänge.

Beim Mapping eines Integrationsbereichs werden überblicksartig die tatsächlichen Geschäftsaktivitäten in der Unternehmung im Zeitablauf dargestellt. Entscheidend für das Verständnis einer Geschäftsaktivität ist dabei die Erfassung realer Folgeketten, einschließlich informaler Kommunikation. ${ }^{2}$ Dazu werden verschiedene Methoden eingesetzt, etwa Interviews, Simulationen sowie die Mitverfolgung und ex-post Analyse abgeschlossener Vorgänge. Ergebnis solcher Erhebungen sind natürlich völlig unterschiedliche Interpretationen der verschiedenen Beteiligten, beispielsweise vom tatsächliche Ablauf eines Entscheidungsprozesses in der Produktneuentwicklung. Deshalb kommt es bei der Analyse darauf an, diese verschiedenen Perspektiven herauszufinden und abzugleichen, d.h. eine gemeinsame Map zu erstellen. Entscheidend ist, daß die tatsächlichen Träger der betrachteten Geschäftsaktivitäten in die Erstellung einer gemeinsamen Map einbezogen werden. Schon dabei werden die Beteiligten aus verschiedenen Unterneh-

1 Vgl. Weick, K.E., The Social Psychology of Organizing, 2nd ed., New York, 1979, S. 140; Argyris, C., Putnam, R., McLain Smith, D., Action Science, San Francisco, London 1985, S. 247-265; McCaskey, M.B., The executive challenge: managing change and ambiguity, Boston, 1982, S. 19; ähnlich vgl. Stalk, G., Hout, Th.M., Competing Against Time, a.a.O., S. $200 \mathrm{ff}$.

2 Vgl. Stalk, G., Hout, Th.M., Competing Against Time, a.a.O., S. 201. 
mensfunktionen und Hierarchiestufen eine neue Sicht des Prozesses entwickeln. ${ }^{1}$

Bei der Definition des Integrationsbereichs hängen Umfang und Detaillierungstiefe vom Inhalt und Aggregationsniveau des betrachteten Problems ab. Vier generische Problemebenen wurden zuvor unterschieden:2 der Produktionsproze $B$, der Auftragsproze $B$, der Entwicklungsproze $B$ und der Managementprozeß. Von der Prozeßebene hängt es ab, wie tief die Unternehmung bei der Betrachtung von Integration analytisch zu dekomponieren ist. Beispielsweise ist ein zur "Verkürzung der Auftragsabwicklungsszeit" definierter, schwerpunktmäßig unternehmensintern orientierter Integrationsbereich genauer in einzelne Geschäftsaktivitäten zu detaillieren, während ein zur "Neuausrichtung auf die Kundengruppen $A, B, C$ " definierter, extern orientierter Integrationsbereich ein höheres Aggregationsniveau hat.

Ergebnis des Mapping ist eine Map der Unternehmensteile, Geschäftsaktivitäten und der sie verbindenden Informationsflüsse (ggf. ebenfalls Materialflüsse). Eine entsprechende Notation wird in Abb. B.2.2 vorgeschlagen. Als Beispiel ist ein Entwicklungsprozeß skizziert, der Geschäftsaktivitäten und Informationsflüsse von der Produktidee bis zur Produktionsreife enthält. ${ }^{3}$

Unternehmensteile als Träger von Geschäftsaktivitäten sind im Rahmen des Integrationsmanagements nicht mehr weiter untergliederte aufbauorganisatorische Teile eines Unternehmens, beispielsweise ein Geschäftsbereich, eine Abteilung, eine Gruppe von Arbeitsplätzen oder ein Arbeits-

1 Vgl. Weick, K.E., Social Psychology of Organizing, a.a.O., S. 69 ff; Stalk, G., Hout, Th.M., Competing Against Time, a.a.O., S.203.

2 Nach Mesarovic, Macko und Takahara waren Echelons, Layers und Strata unterschieden worden. Die Echelons korrespondieren mit dem Unternehmensteil, die Strata mit den Integrationsinstrumenten und die Layer mit dem Geschäftsprozeß. Vgl. Mesarovic, D., Takahara, Y., Theory of Hierarchical, a.a.O. sowie Abschnitt A.2.1.1.

3 Vgl. z.B. Roberts, E.B., Managing Invention and Innovation, in: Research Technology Management, Vol. 31, No. 1, 1988, S. 12; Stalk, G., Hout, Th.M., Competing Against Time, a.a.O., S. 124. 
platz. Jede Zeile des dargestellten Integrationsbereichs entspricht einem Unternehmensteil, der links angegeben ist. Ihm zugeordnet sind die von ihm getragenen Geschäftsaktivitäten.

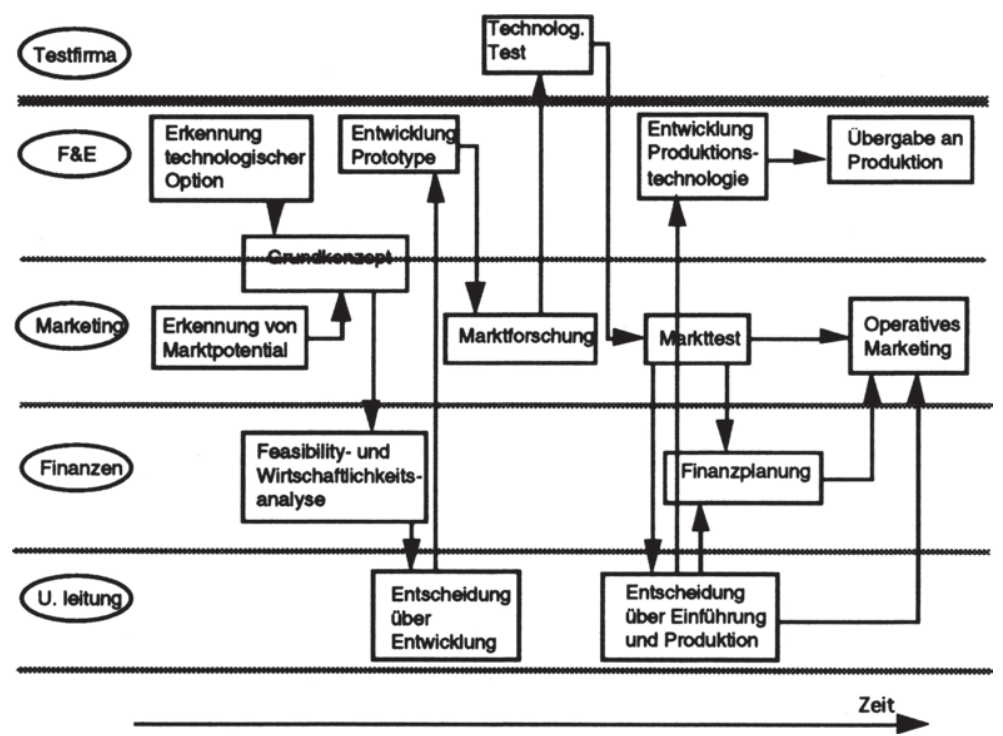

Abb. B.2.2: Map eines Produktinnovationsprozesses

Pfeile symbolisieren Flüsse von Informationen, die nach Vollendung einer Geschäftsaktivität in einem Unternehmensteil in eine folgende Geschäftsaktivität eingehen. Schnittstellen zwischen Unternehmensteilen werden durch graue Trennmarkierungen gekennzeichnet, und zwar schmale innerhalb der Unternehmung und breite bei Einbeziehung von externen Unternehmen oder Unternehmensteilen. Diese Notation wird in der gesamten weiteren Arbeit zur Darstellung von Interdependenzen zwischen Unternehmensteilen bzw. Geschäftsaktivitäten genutzt.

Das Mapping hat als Analyseinstrument gegenüber einer globalen, konzeptionellen Aggregation von Geschäftsaktivitäten zu einer Wertkette folgende Vorteile. Erstens wird explizit zwischen Geschäftsaktivitäten und den sie tragenden Unternehmensteilen unterschieden. Damit kann herausgear- 
beitet werden, wo konkret, d.h. zwischen welchen Unternehmensteilen integrative Verbindungen bestehen (sollen), ohne in der Darstellung Zuständigkeit und Tätigkeit zu vermengen. Zweitens werden durch explizite Einbeziehung der Zeit operationale Meßgrößen (z.B. Zykluszeiten)1 dargestellt, die in der aggregierenden (Wertketten-)Betrachtung von Kosten- oder Qualitätsinformationen verlorengehen können. Drittens werden mit der Orientierung an detaillierten Geschäftsaktivitäten und konkreten Abläufen Integrationspotentiale (Schnittstellenprobleme, Synergiepotentiale) besser identifizierbar als in der abstrakt orientierten Wertkettenanalyse. Insofern ermöglicht das Mapping im Vergleich zu Wertkettendarstellung eine dem Integrationsproblem gemäßere Dekomposition der Unternehmung bzw. eines Geschäftssystems in einzelne Geschäftsaktivitäten.

\subsubsection{Elemente des Integrationsbereichs und integrative Verbindungen}

Unternehmensteile und die von ihnen getragenen Geschäftsaktivitäten sind die Elemente des Integrationsbereichs. ${ }^{2}$ Integration kann auch über Firmengrenzen hinausreichen, insoweit mögen "Unternehmensteile" auch ganze Unternehmungen oder Teile verschiedener Unternehmungen sein. Interdependenzen zwischen diesen Elementen des Integrationsbereichs sind an Gleichartigkeiten und Schnittstellen erkennbar, was durch die Erarbeitung einer gemeinsamen Map wesentlich erleichtert wird. Gleichartigkeiten von zwei oder mehreren Geschäftsaktivitäten begründen Potentiale horizontaler Verflechtung, ${ }^{3}$ die durch Abstimmung der Geschäftsaktivitä-

1 Vgl. Abschnitt B.1.3.1.3.

2 Zur Definition von Unternehmensteilen und Geschäftsaktivitäten vgl. Abschnitt A.2.1.1.

3 Konsistent hierzu unterscheidet Frese drei Formen von Interdependenzen: (1) Interdependenzen aufgrund innerbetrieblicher Leistungsverflechtung, (2) Ressourceninterdependenzen und (3) Marktinterdependenzen. Durch innerbetriebliche Leistungsverflechtung können Interdependenzen zwischen nacheinandergelagerten Unternehmensteilen entstehen, $\mathbf{z} . \mathbf{B}$. zwischen Produktion und Absatz. Diese Interdependenz entspricht hier der Schnittstelle. Demgegenüber liegt eine Überschneidung des Entscheidungsfeldes vor, wenn zwei Unternehmensteile über gleiche Ressourcen oder Märkte verfügen. Diese Interdependenzen entsprechen hier der Gleichartigkeit. Vgl. Frese, E., Organisation, a.a.O., S. 190 ff. 
ten realisiert werden, z.B. eine gemeinsame Nutzung von Ressourcen. ${ }^{1}$ Gleichartigkeiten entsprechen den gebündelten Interdependenzen (pooled interdependency) in Thompsons System von Interdependenzen. Schnittstellen zwischen zwei oder mehreren Geschäftsaktivitäten begründen Potentiale vertikaler Verknüpfung, die gebildet wird bei ablaufbezogenen bzw. Rückkopplungsbeziehungen. Schnittstellen entsprechen insofern Thompsons sequentiellen und reziproken Interdependenzen. ${ }^{2}$

\subsubsection{Horizontale Verflechtungen}

Bei einer horizontalen Verflechtung führen verschiedene Unternehmensteile gleichartige oder zumindest ähnliche Geschäftsaktivitäten gemeinsam oder abgestimmt aus. Gemeinsam optimieren sie damit die Geschäftsaktivitäten, so daß ein synergetischer Effekt entsteht, z.B. Kostenvorteile in der Produktion. ${ }^{3}$ Synergetische Effekte sind möglich, wenn Interdependenzen vorliegen, oder umgekehrt, Interdependenzen zwischen nebeneinanderstehenden Unternehmensteilen liegen vor, wenn eine Abstimmung von Geschäftsaktivitäten einen größeren Beitrag zum Unternehmensziel leistet als die isolierte Führung der Unternehmensteile, z.B. durch gemeinsame Nutzung von Produktionsfaktoren oder gemeinsame Marktbearbeitung. ${ }^{4}$ Ein gemeinsames Vorgehen erscheint nur dann sinnvoll, wenn die Geschäftsaktivitäten der Unternehmensteile gleichartig sind. Beispielsweise

1 Thompson, J.D., Organizations in Action, a.a.O., S. 55; vgl. auch Mintzberg, H., Structuring of Organizations, a.a.O., S. 22 f.

2 Thompson, J.D., Onganizations in Action, a.a.O., S. 55 ff.

3 Riebel betont, daß sowohl verschiedene Produktarten als auch Teilmengen einer Produktart integrativ verbunden sein kömen, d.h. daß sowohl gleichartige Produkte als auch nicht gleichartige Produkte horizontal verflechtbar sind. Damit läge im Hinblick auf die Produkte keine Gleichartigkeit vor. Demgegenüber wird der Gleichartigkeitsbegriff hier weiter gefaßt, d.h. er muß nicht notwendigerweise auf das Produkt bezogen sein, sondem auf irgendeine zur Erstellung von Produkten erforderliche Geschäftsaktivität. Ohne eine so verstandene Gleichartigkeit ist die Bildung einer materiellen oder immateriellen horizontalen Verflechtung sinnlos. Vgl. dazu Ropella, W., Synergie, a.a.O., S. 224 f; Riebel, P., Produktion III: einfache und verbundene, in: HdWW, Band 6, (Hrsg.), Albers, W. u.a., Stuttgart, 1981, S. 298.

4 Vgl. z.B. Frese, E., Organisation, a.a.O., S. 193. 
weist die Vermarktung von Flugreisen Gleichartigkeiten mit der Vermarktung von Ferienhotels oder Mietwagen auf.

Potentiale für eine horizontale Verflechtung können nach verschiedenen Kriterien systematisiert werden, beispielsweise nach der Art der eingesetzten Produktionsfaktoren, ${ }^{1}$ der betrachteten Geschäftsaktivitäten, ${ }^{2}$ der zu verflechtenden Unternehmensteile oder der vorhandenen Interdependenzen. ${ }^{3}$ Die Wahl des Kriteriums beeinflußt das Vorgehen bei der Analyse horizontaler Verflechtungen. ${ }^{4}$ Ziel eines praktischen Integrationsmanagements ist es, die Integration der Geschäftsaktivitäten zu erreichen, deren Träger die Unternehmensteile sind. Sie werden daher, Porter folgend, hier als Systematisierungskriterium gewählt.

Zur Identifikation von Potentialen horizontaler Verflechtung ist somit nach Gleichartigkeiten von Geschäftsaktivitäten zu suchen. Zur Lokalisierung der Gleichartigkeiten sind zunächst nebeneinanderstehende Unternehmensteile zu benennen, deren Geschäftsaktivitäten aufzulisten und zu

1 Jede horizontale Verflechtung beruht auf der gemeinsamen Nutzung von Produktionsfaktoren. Ropella legt ein von ihm so bezeichnetes "synergieorientiertes" Produktionsfaktorensystem vor. Er unterscheidet die Produktionsfaktoren danach, ob sie simultan oder nur sukzessive an der Erstellung zweier Produkte beteiligt sein können. Dieses produktionswirtschaftlich orientierte Faktorsystem stellt eine umfassende Systematik möglicher Arten von Faktoren dar, die gemeinsam genutzt werden können. Ropella, W., Synergie, a.a.O., S. 249, Abb. 41.

2 Porter teilt sein System von 9 Geschäftsaktivitäten in 5 Klassen verschiedenartiger Verflechtungspotentiale auf. Vgl. Porter, M.E., Competitive Advantage, a.a.O., S. 338.

3 Frese unterscheidet nach zwei Kriterien, nämlich nach der Art der zu verflechtenden Unternehmensteile (d.h. ob die Unternehmensteile nach Produkten, Gebieten, Funktionen etc. divisionalisiert sind) und nach der Art der Interdependenz (d.h. ob die Interdependenz sich auf Märkte, Ressourcen oder die Produktion bezieht). Frese, E. Organisation, a.a.O., S. 237.

4 Beispielsweise würde eine Analyse horizontaler Verflechtungen, die nach den eingesetzten Produktionsfaktoren vorgeht, fragen, welche grundsätzlichen Verflechtungspotentiale zwischen welchen Produktionsfaktoren bestehen. Demgegenüber wünde eine Analyse horizontaler Verflechtungen, die nach der Art der Unternehmensteile vorgeht, fragen, welche grundsätzlichen Verflechtungspotentiale zwischen funktional gegliederten Unternehmensteilen, zwischen produktdivisionalen Unternehmensteilen oder zwischen regional gegliederten Unternehmensteilen möglich sind. Dabei erscheint es als schwierig, eine sehr detaillierte Analyse der Verflechtungspotentiale aller Produktionsfaktoren vorzunehmen und zudem eine klare Verbindung zum strategischen Zielsystem herzustellen. Demgegenüber erscheint eine Analyse möglicher Verflechtungspotentiale nach der Art der Unternehmensteile als zu global und kaum operationalisierbar. 
klassifizieren. Schließlich sind Gleichartigkeiten herauszuarbeiten. Diese Schritte werden, solange die $\mathrm{zu}$ betrachtenden Unternehmensteile nicht vorgegeben sind, iterativ mehrfach zu durchlaufen sein. Am Ende des Analyseprozesses steht die Definition eines Integrationsbereichs in Bezug auf Potentiale horizontaler Verflechtung.

Abbildung B.2.3 enthält einen Ausschnitt der Geschäftsaktivitäten einer Fluggesellschaft, einer Autovermietung und eines Hotelkonzerns. Die Basis horizontaler Verflechtungspotentiale sind Gleichartigkeiten. Liegen Gleichartigkeiten vor, können Unternehmensteile durch gemeinsames Handeln Synergien schaffen. Gleichartigkeiten können einerseits direkt zwischen den Geschäftsaktivitäten zweier oder mehrerer Unternehmensteile bestehen.

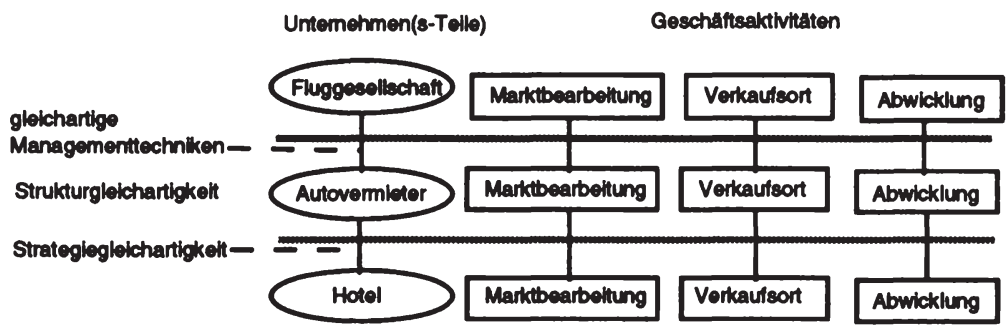

immateriblib horleontaib Veribchtungen

Materible horlsontals Veribchtungen

Abb. B.2.3: Materielle und immaterielle horizontale Verflechtungen

Andererseits ist es möglich, daß die Geschäftsaktivitäten von Unternehmensteilen zwar nicht direkt gleichartig sind, aber indirekt; sie können z.B. grundsätzlich die gleiche Struktur aufweisen (vgl. Abb. B.2.3). Entsprechend werden im folgenden materielle horizontale Verflechtungen (direkt) und immaterielle horizontale Verflechtungen (indirekt) unterschieden. ${ }^{1}$

1 Vgl. Porter, M.E., Competitive Advantage, a.a.O., S. 323. 
Im Beispiel ergeben sich materielle horizontale Verflechtungen aus Möglichkeiten der gemeinsamen Marktbearbeitung (z.B. gemeinsame Anzeigenwerbung, gemeinsame Promotions, gegenseitige Anerkennung von Frequent Flyer Miles oder Mitgliedschaftsprogrammen), aus gemeinschaftlich betriebenen Verkaufsstellen (an Flughäfen, in Hotels oder durch gemeinsames Auftreten gegenüber Agenturen oder Reisebüros) und aus gemeinschaftlich betriebener Abwicklung (gemeinsames Kundeninformations- und Reservierungssystem, gemeinsame Zahlungsabwicklung).

Immaterielle horizontale Verflechtungen beruhen auf indirekten Gleichartigkeiten, wie Managementtechniken, Geschäftsstrukturen oder Strategien. Fluggesellschaften und Autovermieter haben ähnliche Geschäftsprobleme, etwa Kapazitätsauslastung bei schwankender Nachfrage, oligopolistische Preisbildung und hohes Volumen einzelner Transaktionen. Entsprechend sind Managementtechniken übertragbar, z.B. ein preisdifferenzierendes Yield-Management, Führungsstil u.v.a. Ein bedeutendes neues Potential immaterieller horizontaler Verflechtungen ist die gemeinsame Nutzung von Kunden- und Produktinformationen aus Datenbanken, die mehrere Unternehmen als Entscheidungsgrundlage nutzen können. ${ }^{1}$

Die Identifikation von Gleichartigkeiten von Geschäftsaktivitäten erfordert eine genaue Analyse der Faktoren, die für die einzelne Geschäftsaktivität von zentraler Bedeutung sind. Gleichartigkeiten müssen in Bezug auf diese Faktoren vorliegen, im Fall des Marketings etwa die (wirklich) gleichen Kunden. Dazu ist die Marktsegmentierung der einzelnen beteiligten Unternehmen oder Unternehmensteile genau zu vergleichen, etwa in Bezug auf Regionen, Kunden, Produkte oder Technologien. Gleichartigkeiten im Marketing beruhen auf einer gleichartigen Elastizität der Marketinginstrumente in den Segmenten, die gemeinsam genutzt werden. In der Beschaffung beziehen sich Gleichartigkeiten auf die zu beschaffenden Rohstoffe, die Lieferanten, die Technologien oder Regionen. In der Logistik ist

1 Vgl. Abschnitt C.2.3.3. 
es nicht hinreichend, daß Regionen und Routen gleichartig sind, darüber hinaus sind auch die Transportfrequenz und die Beschaffenheit des transportierten Guts zu beachten. In der Produktion können gleichartige Produktionstechnologien, Prozesse, Produkte oder Einsatzfaktoren vorliegen. In der Forschung und Entwicklung sind Gleichartigkeiten auf das Feld bzw. den Gegenstand der Forschung, Grundausrichtung der Forschung (F oder E), den angestrebten Zeithorizont und Neuerungsgrad $\mathrm{zu}$ beziehen. Im Kundendienst sind gleichartiger Gegenstand, Servicegrad und regionale Aspekte von Interesse. Beim Personal sind gleichartige Fähigkeitspotentiale und gleichartige Werte von Bedeutung. Für Finanzabteilung und Verwaltung sind gleichartiger oder sich ergänzender Finanzierungs- und Verwaltungsbedarf von Bedeutung, etwa im Hinblick auf das Muster des Kapitalbedarfs oder des Berichtswesens von Bedeutung.

Während die Interdependenzen bei horizontalen Verflechtungen auf Gleichartigkeiten von Geschäftsaktivitäten beruhen, sind sie bei vertikalen Verknüpfungen durch Schnittstellen zwischen nacheinandergelagerten Geschäftsaktivitäten begründet.

\subsubsection{Vertikale Verknüpfungen}

Die Logik vertikaler Verknüpfungen ist es, Reibungsverluste zwischen nacheinandergelagerten, interdependenten Geschäftsaktivitäten abzubauen, und zwar durch eine verbesserte Abstimmung der Geschäftsaktivitäten bzw. der sie tragenden Unternehmensteile. Interdependenzen zwischen hintereinandergelagerten Unternehmensteilen ergeben sich aus dem Material- und Informationsfluß an den Schnittstellen zwischen den Unternehmensteilen. Beispielsweise könnten Produktion und Distribution nahezu isoliert voneinander arbeiten, wenn für alle Produkte hinreichende Lagerhaltung zugelassen würde. Um dafür entstehende Kosten-, Qualitätsund Zeitnachteile zu reduzieren, ist eine engere vertikale Verknüpfung der Produktion mit der Distribution erforderlich. 
In Abb. B.2.4 ist ein Auftragsprozeß der Herstellung und Distribution eines Food-Markenartikels skizziert.

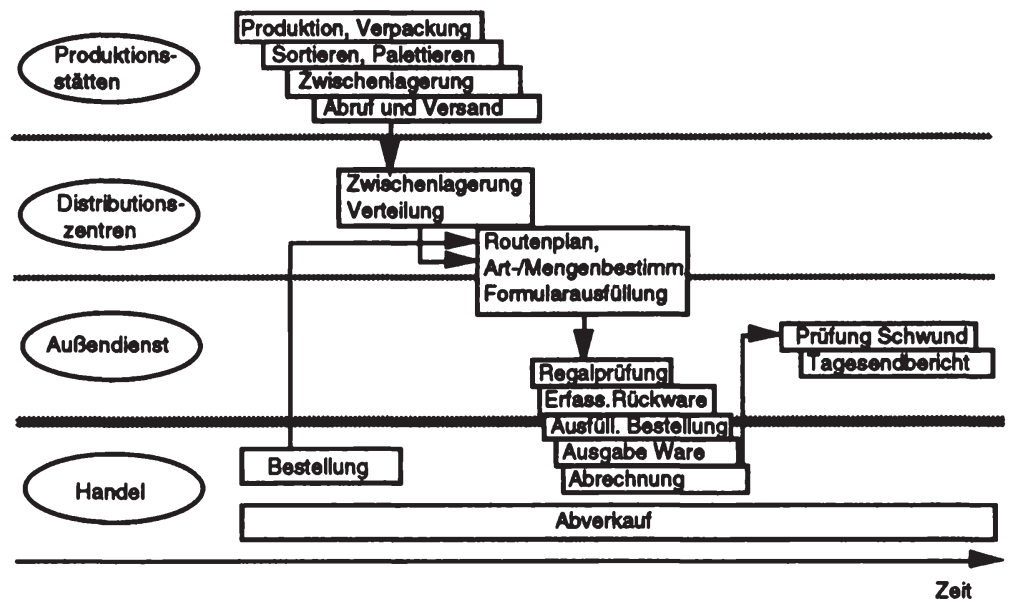

Abb. B.2.4: Auftragsprozeß Herstellung und Distribution eines Markenartikels

Schnittstellen zwischen Geschäftsaktivitäten ergeben sich aus dem Material- und Informationsfluß; sie werden als Pfeile dargestellt. Der Pfeil symbolisiert das Material oder die Informationen, die nach Vollendung einer Geschäftsaktivität in einem Unternehmensteil in eine folgende Geschäftsaktivität eingehen. Schnittstellen zwischen Geschäftsaktivitäten innerhalb eines Unternehmensteils sind lediglich durch verschobene Blöcke dargestellt, wobei die Verschiebung den Zeitablauf angeben soll. Die benötigte Zeit an der Schnittstelle zwischen zwei Geschäftsaktivitäten hängt dabei davon $a b$, welche Integrationsinstrumente zur vertikalen Verknüpfung der diese Geschäftsaktivitäten tragenden Unternehmensteile gewählt werden.

Schnittstellen zwischen Unternehmensteilen können unterschieden werden in Binnenschnittstellen, d.h. Schnittstellen innerhalb eines Unternehmensteils oder zwischen Unternehmensteilen, und Randschnittstellen, d.h. Schnittstellen zwischen Unternehmungen. Zur graphischen Unterscheidung zwischen diesen Schnittstellenarten werden in dieser Arbeit 
Randschnittstellen durch breite Trennmarkierungen, Binnenschnittstellen zwischen Unternehmensteilen mit schmalen Trennmarkierungen dargestellt. Abhängig von der Art der Schnittstelle sind völlig verschiedene kontraktuelle, organisationale und technische Integrationsmöglichkeiten gegeben. Durch veränderte kontraktuelle Integration kann jedoch auch aus einer Binnenschnittstelle eine Randschnittstelle werden.

\subsection{Situative Bedingungen im Integrationsbereich}

Vertikale Verknüpfungen wie horizontale Verflechtungen werden durch Einsatz geeigneter Integrationsinstrumente hergestellt. Der Möglichkeitsraum für ihre Auswahl und ihre Eignung ist nicht invariant in Bezug auf die situativen Bedingungen im Integrationsbereich (vgl. Abb. B.2.5).

Die Erkenntnis, daß die Vorteilhaftigkeit organisatorischer Handlungsoptionen situativ bedingt ist, bildet den paradigmatischen Kern des situativen Ansatzes der Organisationstheorie. ${ }^{1}$ Danach hängt die Effizienz von Organisationsstrukturen von ihrer zweckmäßigen Ausrichtung auf die Situation ab. So wird vielfach auf einen Zusammenhang zwischen Organisationsgröße und Koordinationsmechanismen hingewiesen. ${ }^{2}$ Der Begriff der Situation als offenes Konzept bedarf der Ausfüllung in Abhängigkeit von der Fragestellung, hier der Integrationsentscheidung. ${ }^{3}$

1 Eine Übersicht geben z.B. Kieser, A., Kubicek, H., Organisation, a.a.O., S. 47 ff.

2 Vgl. Kieser, A., Kubicek, H., Organisation, a.a.O., S. 271 ff und dort angegebene empirische Studien.

3 Vgl. Kieser, A., Kubicek, H., Organisation, a.a.O., S. 217. 


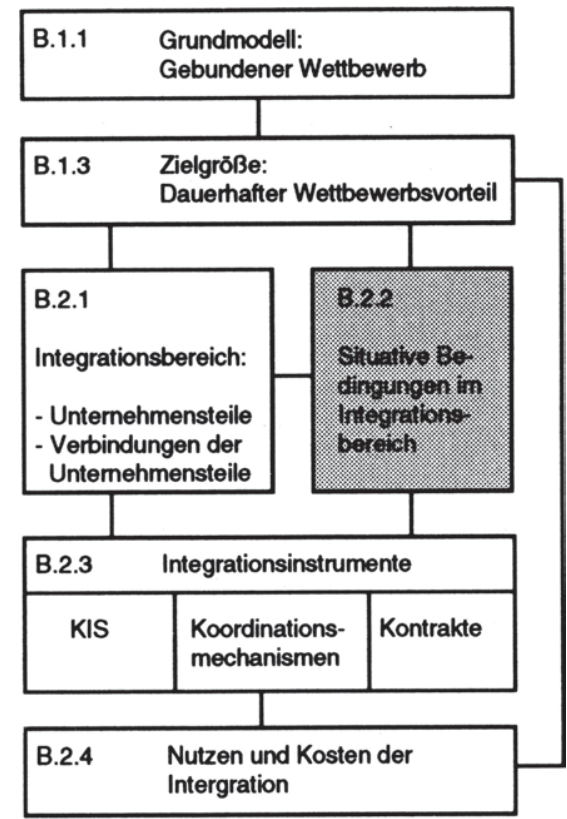

Abb. B.2.5: Stellung der situativen Bedingungen im Bezugsrahmen

Nachstehend wird ein einfacher multivariater Ansatz ${ }^{1}$ aus den drei situativen Bedingungen Volumen, Unsicherheit und Spezifität entwickelt. Es ist jeweils der Begriff genauer zu definieren sowie, die Relevanz für die Integrationsentscheidung aufzuzeigen. ${ }^{2}$ Die Auswirkung der situativen Bedingungen auf den Einsatz der Integrationsinstrumente wird im Abschnitt 2.3.2 diskutiert.

1 Zur Unterscheidung multivariater und monovariater Ansätze zur Beschreibung der Situation vgl. Kieser, A., Kubicek, H., Organisation, a.a.O., S. 213 ff.

2 Vgl. Kieser, A., Kubicek, H., Organisation, a.a.O., S. 218 


\subsubsection{Volumen}

Als Volumen wird die "Mächtigkeit" der im Integrationsbereich abzuwikkelnden Geschäftsaktivitäten bezeichnet. Das Volumen der Geschäftsaktivitäten ergibt sich als Produkt aus der absoluten Anzahl der Geschäftsaktivitäten, der zeitlichen Frequenz ihrer Abwicklung und ihrer jeweiligen Komplexität.

Die Anzahl der Geschäftsaktivitäten ist bedingt von einer Reihe interner und externer Faktoren. Interne Faktoren sind z.B. die Unternehmensgröße, ausgedrückt etwa in der Anzahl der Mitarbeiter, den Produktionsmengen oder der Anzahl der Produktlinien. Externe Faktoren sind z.B. das Marktvolumen oder der Marktanteil.1

Die Frequenz einer Geschäftsaktivität ist der reziproke Wert ihrer Zeitdauer. Sie gibt an, wie oft die Unternehmung eine Geschäftsaktivität durchführt. Eine hohe Frequenz deutet dabei tendenziell auf im Zeitablauf wiederkehrende Geschäftsaktivitäten hin.

Die Komplexität der Geschäftsaktivitäten im Integrationsbereich ist aus der einzelnen Geschäftsaktivität erklärbar. Sie ist um so höher, je mehr Variable oder Informationen erforderlich sind, um die Geschäftsaktivität oder das zugrundeliegende Produkt zu beschreiben, je intrikater die Beziehungen zwischen jenen Variablen ist und je genauer die Geschäftsaktivität oder das Produkt beschrieben werden müssen. ${ }^{2}$ Die Genauigkeit der Beschreibung hängt ihrerseits ab von physischen bzw. technischen Spezifikationen und der Kürze des Zeitintervalls, in der gegebene Informationen

1 Vgl. z.B. Kieser, A., Kubicek, H., Organisation, a.a.O., S.261 ff; Schanz, G., Organisationsgestaltung, a.a.O., S. 266 ff; Mintzberg, H., Structuring of Organizations, a.a.O., S. $230 \mathrm{ff}$.

2 Vgl. Schanz, G., Organisationsgestaltung, a.a.O., S. 301. Zeit und Verschiedenartigkeit verursachen Komplexität, weil sie zusätzliche Variablen erfordern. Kürzere Zeitintervalle erfordern die Angabe aller Daten für eine größere Zahl von Zeitfeldern, während größere Verschiedenartigkeit zwischen den Produkten c.p. die Unterscheidung einer höheren Zahl von Parametern erfordert. 
weiterverarbeitet werden müssen. Die so beschriebene Komplexität zieht ein eher mengenmäßiges als qualitatives Problem der Informationsverarbeitung nach sich. Komplexität wird nicht als Unklarheit über die Natur von Beziehungen zwischen Variablen angesehen, ${ }^{1}$ sondern als Unsicherheit. ${ }^{2}$

Das Problem, wie Geschäftsaktivitäten im Integrationsbereich in strategisch zweckmäßiger Weise integriert werden können, hat in einem hochvolumigen oder geringvolumigen Integrationsbereich eine völlig andere Struktur. Mit dem Volumen von Geschäftsaktivitäten im Integrationsbereich nimmt der Integrationsaufwand zu. Mehr Informationen (pro Zeiteinheit) müssen verarbeitet, mehr Entscheidungen getroffen werden. ${ }^{3}$ Beispiel eines hohen Volumens von Geschäftsaktivitäten ist die Reservierung von Flugreisen. In einem globalen Reservierungssystem sind die verschiedensten Flugtarife und Sitzverfügbarkeiten nach Klassen, Fluggesellschaften und Routen gespeichert. So enthält das Flugreservierungssystem SABRE 45 Millionen verschiedene Flugtarife, nimmt monatlich 40 Millionen Änderungen der Tarife auf, wickelt 2000 Transaktionen pro Sekunde ab und erstellt 500.000 Kundendokumente pro Tag. ${ }^{4}$ Beispiel eines niedrigen Volumens von Geschäftsaktivitäten ist der Aufkauf oder die Fusion von Unternehmungen. Aus Sicht der aufgekauften Unternehmung ist die Geschäftsaktivität einmalig, und selbst große Investmentbanken betreuen nur eine kleine Zahl von Mergers und Acquisitions, wenige erreichen 100 Transaktionen pro Jahr. Dabei sind die einzelnen Transaktionen sehr verschieden voneinander. In beiden Fällen sind die Potentiale der vertikalen Verknüpfung und horizontalen Verflechtung erheblich. Aufgrund des unterschiedlichen Volumens sind jedoch die eingesetzten Integrationsinstrumente höchst verschieden, beispielsweise ein strukturiertes Kommunikati-

1 Vgl. z.B. Kieser, A., Kubicek, H., Organizational Concept, a.a.O., S. 168.

2 Vgl. Abschnitt 2.2.2.

3 Whisler, Th.L., Man, Organization and Computer, a.a.O., S. 247, S. 249 ff.

4 Vgl. Hopper, M.D., Rattling SABRE - New Ways to Compete on Information, in: Harvard Business Review, Vol. 68, 1990, S. 120. 
ons- und Informationssystem einerseits und im wesentlichen ein gut geflochtenes Netz von Bekanntschaften und Beziehungen andererseits.

\subsubsection{Unsicherheit}

Die Unsicherheit wird häufig als das Hauptproblem der Strategie und Organisation von Geschäftsaktivitäten angesehen. ${ }^{1}$ Läge keine Unsicherheit über die Geschäftsaktivitäten der Unternehmung vor, wäre das Eingehen strategischer Commitments problemlos. Je ausgeprägter jedoch Unsicherheit vorliegt, desto mehr Informationen müssen noch während der Ausführung von Geschäftsaktivitäten verarbeitet werden. ${ }^{2}$ Die Dauerhaftigkeit der Wettbewerbsposition ist gefährdet durch Veränderungen des Datenkranzes, also der weiteren Umwelt der Unternehmung einerseits, und durch das Verhalten von Konkurrenten, Mitarbeitern, Lieferanten und Kunden andererseits. Anhand der Quellen der Unsicherheit können zwei Arten der Unsicherheit unterschieden werden: die Umweltunsicherheit und die Verhaltensunsicherheit. ${ }^{3}$ Dabei ergibt sich die Umweltunsicherheit aus der Art der Produkte bzw. Geschäftsaktivitäten im Integrationsbereich sowie aus der Schwierigkeit der Prognose, Bewertung und Messung zukünftiger Ereignisse. ${ }^{4}$ Demgegenüber resultiert die Verhaltensunsicherheit aus mangelndem Vertrauen und asymmetrischer Informationsverteilung zwischen den Parteien. 5

1 Vgl. Alchian, A., Uncertainty, Evolution, and Economic Theory, in: Journal of Political Economy, Vol. 58, 1950, S. 212; Thompson, J.D., Organizations in Action, a.a.O., S.159; Koopmans, T., Three Essays on the State of Economic Science, New York, 1957, S. 147; Allaire, Y., Firsirotu, M.E., Coping with Strategic Uncertainty, in: Sloan Management Review, Vol. 31, Spring 1989, S. 7.

2 Galbraith, J.R., Complex Organizations, a.a.O., S. 4.

3 Vgl. Mises, L. von, Human Action, 3. Nachdruck, Chicago, 1949, S.110 ff; Williamson, O.E., Economic Institutions, a.a.O., S. 58.

4 Vgl. z.B. Ciborra, C., Reframing the Role of Computers in Organizations - The Transaction Costs Approach, in: Office: Technology and People, Vol. 3, 1987, S. 17 - 38, hier: S. 26.

5 Vgl. Ciborra, C., Reframing the Role of Computers, a.a.O., S. 26. 
Die Umweltunsicherheit oder natürliche Unsicherheit resultiert aus der prinzipiellen Unvorhersehbarkeit zukünftiger Ereignisse. Sie ist besonders abhängig von der Dynamik der Umwelt ${ }^{1}$. Die Umweltdynamik kann anhand der Häufigkeit, Stärke sowie RegelmäBigkeit von Veränderungen beschrieben werden. ${ }^{2}$ Abhängig davon können potentielle Zukunftszustände in ihrer Gesamtheit und die jeweilige Wahrscheinlichkeit ihres Eintretens mit mehr oder weniger Unsicherheit prognostiziert werden. ${ }^{3}$ Die Unsicherheit leitet sich dabei aus der Variabilität und Schwierigkeit der Geschäftsaktivitäten im Integrationsbereich ab. ${ }^{4}$ Die Variabilität kann als die Anzahl der Ausnahmefälle in einem Unternehmensteil operationalisiert werden. Die Schwierigkeit einer Geschäftsaktivität ergibt sich aus der Analysierbarkeit und der Vorhersehbarkeit der Lösungs- bzw. Arbeitsmethoden. Möglichkeiten zur Operationalisierung dazu sind (1) das Ausmaß der Komplexität der Suchprozesse, (2) der Zeitaufwand zum Erdenken der Problemlösung5, (3) das Ausmaß, in dem Aufgaben bzw. Prozesse wißbare Ergebnisse zeitigen, ${ }^{6}$ oder die Zeit bis Ergebnisse bekannt werden.

1 Kieser, A., Kubicek, H., Organisation, a.a.O., S.323. Zur Umweltdynamik als Kontingenzfaktor vgl. Mintzberg, H., Structuring of Organizations, a.a.O., S. 268 und S. 270 ff; Schanz, G., Organisationsgestaltung, a.a.O., S. 303 f; Williamson, O.E., Economic Institutions, a.a.O., S. $56 \mathrm{ff}$.

2 Vgl. Child, J., Organizational Structure, Environment, and Performance: The Role of Strategic Choice, in: Sociology, Vol. 5, 1972, S. 3.

3 Zurückgehend auf Knight, wird in der (entscheidungstheoretischen) Literatur häufig noch zwischen Risiko und Unsicherheit differenziert, wobei Risiko vorliegt, wenn Wahrscheinlichkeiten für die Entscheidungsalternativen angegeben werden können, und Unsicherheit, wenn dies nicht der Fall ist. Vgl. Knight, F., Risk, Uncertainty, and Profit, Boston, 1921. Adam, D., Kurzlehrbuch Planung, a.a.O., S. 84 ff unterscheidet Unsicherheit, bei der objektive, subjektive und keinerlei Wahrscheinlichkeiten angegeben werden können, ähnlich March, J.G., Simon, H.A., Organizations, a.a.O., S. 113. Eine Kritik dieser Ansätze gibt Ellsberg, D., Risk, Ambiguity and the Savage Axioms, in: Quarterly Journal of Economics, Vol. 75, 1961, S. $643 \mathrm{ff}$.

4 Zum folgenden vgl. Van de Ven, A., Delbecq, A., Koenig, R. Jr., Determinants of Coordination Modes Within Organizations, in: American Sociological Review, Vol. 41, 1976, S. 324.

5 Vgl. Perrow, C., A Framework for Comparative Organizational Analysis, American Sociological Review, 1967, Vol. 32, S. 194 - 208.

6 Vgl. Thompson, J.D., Organizations in Action, a.a.O.; Burns, T., Stalker, G.M., The management of innovation, London, 1961. 
Die Verhaltensunsicherheit beruht demgegenüber auf der Unklarheit darüber, wie sich Entscheidungsträger künftig verhalten werden. ${ }^{1}$ Williamson betont die Bedeutung des Problems der Verhaltensunsicherheit, aufbauend auf der Annahme, daß Entscheidungsträger im Wirtschaftsleben im Durchschnitt opportunistisch $\mathrm{zu}$ ihren eigenen Gunsten handeln. Sollten dabei langfristige einseitige Abhängigkeitsverhältnisse zwischen Vertragsparteien bestehen, wird es aufgrund des Problems der Verhaltensunsicherheit erforderlich sein, Institutionen zu bilden, die dem opportunistischen Ausbeuten des Abhängigkeitsverhältnisses entgegenwirken. Eine solche sehr weitgehende Institution ist die kontraktuelle Integration der Vertragsparteien in eine Firma. Das Ausmaß an Verhaltensunsicherheit zwischen nicht kontraktuell integrierten Parteien wird situationsspezifisch sehr verschieden sein. So reduziert die Länge einer bestehenden Zusammenarbeit und gute Erfahrungen während der Zusammenarbeit die Verhaltensunsicherheit durch aufgebaute Reputationseffekte. Die Reputation einer Unternehmung, daß sie sich gegenüber Lieferanten, Abnehmern usw. nicht opportunistisch verhält, auch wenn sie dies könnte, wird oft wertvoller sein als kurzfristige Gewinne aus opportunistischem Verhalten, z.B. dem einseitigen Auslegen von Vertragslücken. ${ }^{2}$ Neben der vergangenen $\mathrm{Zu}$ sammenarbeit ist auch der zukünftige Zeithorizont entscheidend: "what makes it possible for cooperation to emerge is that players might meet again".3 Je langfristiger der zukünftige Zeithorizont der Zusammenarbeit gegenseitig projektiert ist, desto geringer wird die Verhaltensunsicherheit sein.

1 Vgl. Williamson, O.E., Economic Institutions, a.a.O., S. 57 ff.

2 Vgl. Kreps, D., Wilson, R., Reputation and Imperfect Information, in: Journal of Economic Theory, Vol. 27, 1982, S. 253; Cremer, J., Cooperation in Ongoing Organizations, in: Quarterly Journal of Economics, Vol. 101, 1986, S. 33 - 50.

3 Axelrod, R., The Evolution of Cooperation, New York, New York, 1984, S. 12. 


\subsubsection{Spezifität}

Die Spezifität eines Guts oder Faktors ist das Gegenteil seiner Fungibilität.1 Ein Faktor ist um so spezifischer, je größer der Wertverlust wäre, müßte der Faktor verkauft anstatt weitergenutzt werden. ${ }^{2}$ Williamson unterscheidet vier Formen von Spezifität, die räumliche, die physische, die Fähigkeitenoder Personalspezifität und die Lieferanten- bzw. Kundenspezifität. ${ }^{3}$ Damit soll charakterisiert werden, warum der Faktor jeweils spezifisch ist. Beispielsweise mag eine Spezialmaschine auf ein Produkt ausgerichtet sein, für das im wesentlichen nur ein Abnehmer existiert, womit physische und Kundenspezifität vorläge. Die hohe Bedeutung der Spezifität für die Wahl der Integrationsinstrumente, hier insbesondere der kontraktuellen Integration, wird unmittelbar deutlich. Im Falle einer solchen Abhängigkeit wird der Lieferant eine kontraktuelle Absicherung anstreben.

Es erhebt sich die Frage nach dem Unterschied von Spezifität und Starrheit

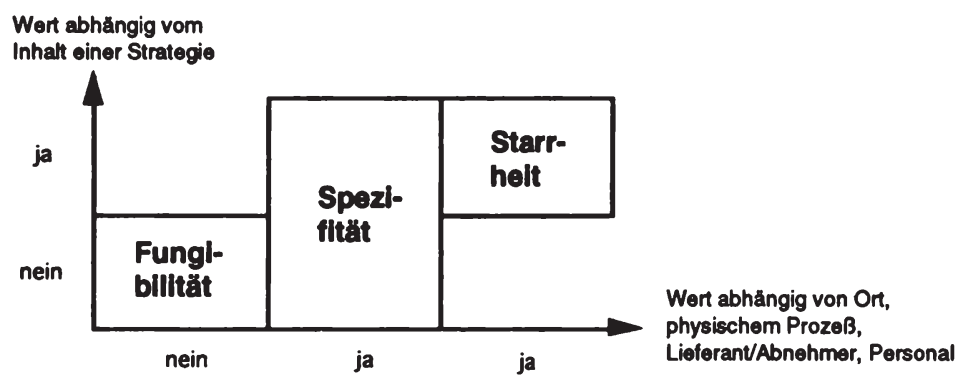

Abb. B.2.6: Faktorstarrheit, Faktorspezifität und Faktorfungibilităt

1 Vgl. Antonelli, C., Dall'economia industriale all'organizzazione industriale, in: Economia Politica, Vol. 2, 1987, S. 285 ff.

2 Vgl. Williamson, O.E., Economic Institutions, a.a.O., S. $52 \mathrm{ff}$.

3 Vgl. Williamson, O.E., Economic Institutions, a.a.O., S. 55. Als Lieferanten- und Kundenspezifität wurde der Williamsonsche Begriff der gewidmeten Vermögensgegenstände (dedicated assets) übersetzt, um anzudeuten, wodurch gewidmete Faktoren zu spezifischen Faktoren werden. 
von Faktoren. Als starrer Faktor wurde ein dauerhafter Potentialfaktor bezeichnet, der sowohl nur für eine bestimmte strategische Option eingesetzt werden kann als auch nicht ohne weiteres veräußerlich ist. Starrheit ist ein Spezialfall der Spezifität, welche durch den Verlust bei Veräußerung von der Fungibilität abgegrenzt wurde (vgl. Abb. B.2.6).

Es wurde gezeigt, daß Commitments einer einzelnen Unternehmung mit Marktaustrittskosten für eine Branche vergleichbar sind. ${ }^{1}$ Caves und Porter zeigen, daß Austrittskosten wie Commitments wesentlich von Investitionen in Faktoren abhängen, die in jeder alternativen Verwendung einen geringeren Wert erbrächten als in der vorgesehenen Verwendung. ${ }^{2}$ Hinsichtlich der "alternativen Verwendung" wird hier jedoch in einen Strategiewechsel und einen Eigentümerwechsel unterschieden, und dies macht den Unterschied der zusammenhängenden Begriffe starr und spezifisch aus. Ein Faktor kann spezifisch im Hinblick auf eine Technologie sein, mag jedoch nicht starr im Hinblick auf eine Strategie sein.

Die Einflußfaktoren Volumen, Unsicherheit und Spezifität bilden ein Gerüst zur Beschreibung der situativen Bedingungen im Integrationsbereich. Sie sind in Abb. B.2.7 zusammenfassend systematisiert. Die Zusammenhänge zwischen situativen Bedingungen und dem Einsatz der Integrationsinstrumente werden im Abschnitt B.2.3.2 diskutiert.

1 Der Begriff "Commitment" kann übersetzt werden als Bindung, aber auch als Verpflichtung. Bindung ist die Wirkung von Commitments in starre Faktoren, daB diese nicht für andere strategische Optionen eingesetzt werden können. Verpflichtung geht mit Commitments einher, weil aus Verlusten bei Veräußerung das Problem der Abschöpfbarkeit erwächst. Vgl. Klein, B., Crawford, R., Alchian, A.A., Vertical Integration, a.a.O., S. 297 f. Gegen Abschöpfung muß sich die Unternehmung institutionell absichem. Vgl. Williamson, O.E., Economic Institutions, a.a.O., S. 52 f. Vgl. auch Kapitel B.1.

2 Vgl. Caves, R., Porter, M.E., Barriers to Exit, in: Essays in Industrial Organization in Honor of Joe S. Bain, (Hrsg.), Masson, R., Qualls, P., Cambridge, Massachusetts, 1976, S.40. 


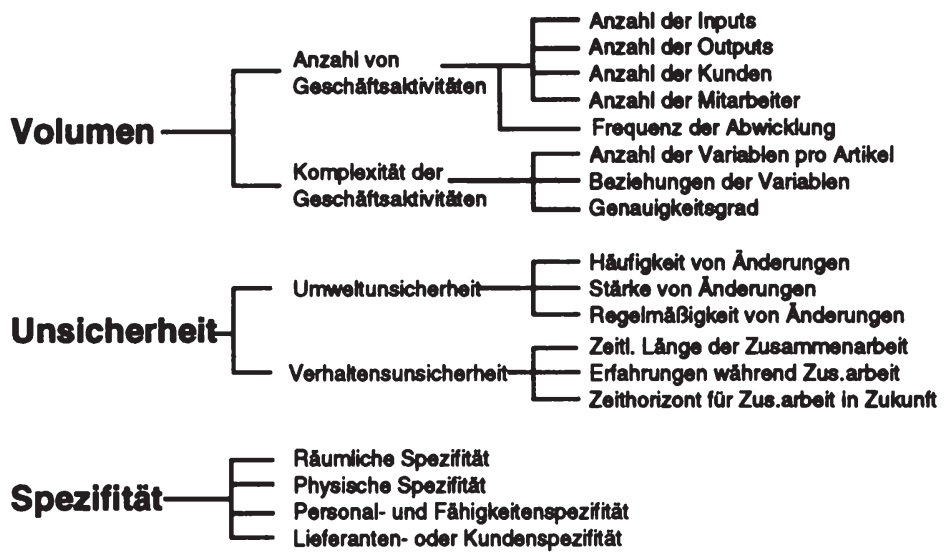

Abb. B.2.7:

Situative Bedingungen im Integrationsbereich

\subsection{Instrumente der Integration}

Die Instrumente des Integrationsmanagements sind die Kommunikationsund Informationssysteme (KIS), die Koordinationsmechanismen und die Kontrakte. Die Integrationsinstrumente sollen so eingesetzt werden, da $\beta$ die Geschäftsaktivitäten im Integrationsbereich unter den gegebenen situativen Bedingungen in strategisch zweckmäßiger Weise integriert werden können (vgl. Abb. B.2.8).

Im folgenden Abschnitt wird für jedes Integrationsinstrument eine Klassifikation erarbeitet, die den bei Integrationsentscheidungen möglichen Alternativenraum abbildet. Darauf aufbauend werden im Abschnitt 2.3.2 die grundsätzlichen Beziehungen der einzelnen Integrationsinstrumente zu den situativen Bedingungen skizziert. 


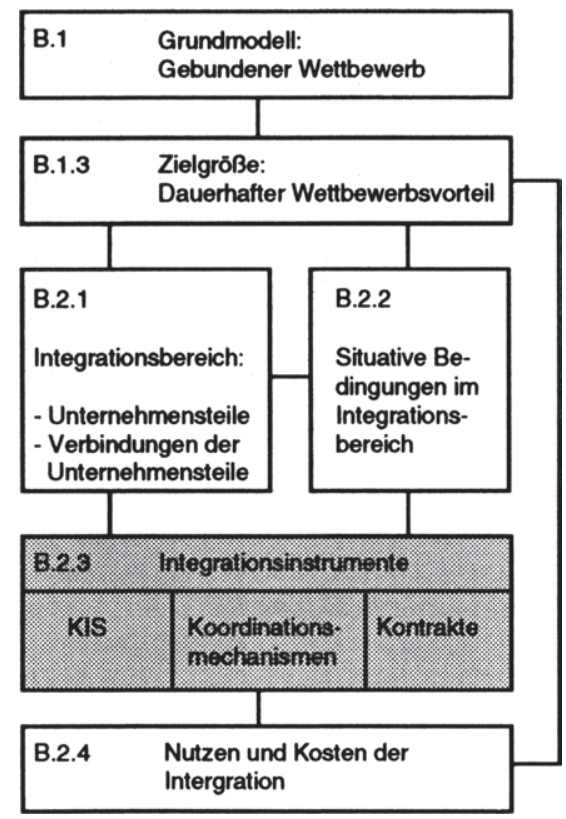

Abb. B.2.8: Stellung der Integrationsinstrumente im Bezugsrahmen

2.3.1 Klassifikation der Integrationsinstrumente

\subsubsection{Kommunikations- und Informationssystem (KIS)}

Die horizontale Verflechtung zielt auf die gemeinsame Ausführung gleichartiger Geschäftsaktivitäten wie die vertikale Verknüpfung auf den Abbau von Schnittstellen. Beides setzt den Austausch von Informationen voraus. Medium des Austauschs von Informationen sind die Kommunikationsund Informationssysteme (KIS). Sie können integrative Verbindungen von Unternehmensteilen bilden, entscheidend bestimmen oder unterstützen. So bildet eine gemeinsam genutzte Datenbank eine horizontale Verflechtung zwischen den Nutzern, wie ein Distributionssystem die vertikale Verknüpfung mit dem Handelskanal entscheidend bestimmt. 
KIS können unterschieden werden anhand der Problemstruktur der Aufgabe, für die sie eingesetzt werden, und nach der Management-Aktivität ${ }^{1}$, d.h. der im Integrationsbereich betrachteten Ebene des Geschäftsprozesses ${ }^{2}$ (vgl. Abb. B.2.9).

Bei der Problemstruktur ist zwischen einer strukturierten, halbstrukturierten und unstrukturierten Ausprägung zu unterscheiden. ${ }^{3}$ Geschäftsaktivitäten sind strukturiert in dem Ausmaß, in dem für sie Routineabläufe oder Prozeduren definiert werden können, so daß nicht jedesmal de novo über ihre Abwicklung entschieden werden muß. Diese Problemstruktur tritt insbesondere dann auf, wenn Geschäftsaktivitäten wiederholt abgewickelt werden. Geschäftsaktivitäten sind unstrukturiert, wenn sie neu oder selten

1 Anthonys Unterscheidung der Management-Aktivitäten nach Strategischer Planung, Management Planung und Steuerung (Management Control) und operativer Planung und Steuerung (Operational Control) kann als klassisch gelten. Strategische Planung umfaßt Entscheidungen über Ziele der Unternehmung, über die Faktorallokation zum Erreichen dieser Ziele und die Grundentscheidungen über die Beschaffung, Nutzung und Veräußerung von Faktoren. Managementplanung und -steuerung (Management Control) umfaßt das Sicherstellen der Beschaffung der Faktoren und deren effektiven und effizienten Einsatz im Hinblick auf das Unternehmensziel. Die operative Planung und Steuerung (Operational Control) umfaßt das Sicherstellen der effektiven und effizienten Ausführung spezifischer Aufgaben. Vgl. Anthony, R.N., Planning and Control Systems: A Framework for Analysis, Cambridge: Harvard University Graduate School of Business Administration, Studies in Management Control, 1965, S. 24 ff. Ähnlich ist Kochs System strategischer, taktischer und operativer Unternehmensplanung. Vgl. Koch, H., Integrierte Unternehmensplanung, a.a.O., S. 34 ff. In der Systematik dieser Arbeit wurden die verschiedenen Management-Aktivitäten bereits als Ebenen des Geschäftsprozesses bezeichnet, denen besser als den Management-Aktivitäten konkrete Zeitzyklen zugeondnet werden können.

2 Vgl. Gorry und Scott Morton, 1971, zitiert bei Keen, P.G.W., Scott Morton, M.S., Decision Support Systems, Reading, Massachusetts, 1978, S. 81 ff. Der Ansatz hat inzwischen weite Verbreitung gefunden. Vgl. z.B. Scheer, A.-W., EDV-orientierte Betriebswirtschaftslehre, a.a.O., S. 72; Awad, E.M., Management Information Systems, Concepts, Structure and Applications, California, 1988, S. 270. In der Literatur wird jedoch auch eine Fülle anders strukturierter Auflistungen und Taxonomien von Kommunikationsund Informationssystemen vorgeschlagen. Vgl. z.B. Szyperski, N., Computergestützte Informationssysteme, a.a.O., Sp. 1901 ff; McLeod, R.Jr., Management Information Systems. A Study of Computer Based Information Systems, 4. Auflage, New York 1990, S. 28.

3 Vgl. zum folgenden Keen, P.G.W., Scott Morton, M.S., Decision Support Systems, a.a.O, S. 11 und S. $81 \mathrm{ff}$. 
sind, so daß nur grobe methodische Rahmen existieren, um die Entscheidungen über die Abwicklung der Geschäftsaktivitäten zu prädisponieren. ${ }^{1}$

In dem von Problemstruktur und Problemebene aufgespannten Raum können die wesentlichen Arten von Kommunikations- und Informationssystemen definiert werden: Abwicklungssysteme, Management-Informationssysteme, Management Support Systems, Expertensysteme sowie reine Kommunikations- und Büroautomatisierungssysteme. Die Unterscheidung dieser Arten ist mehr konzeptionell als technologisch oder physisch bestimmt.

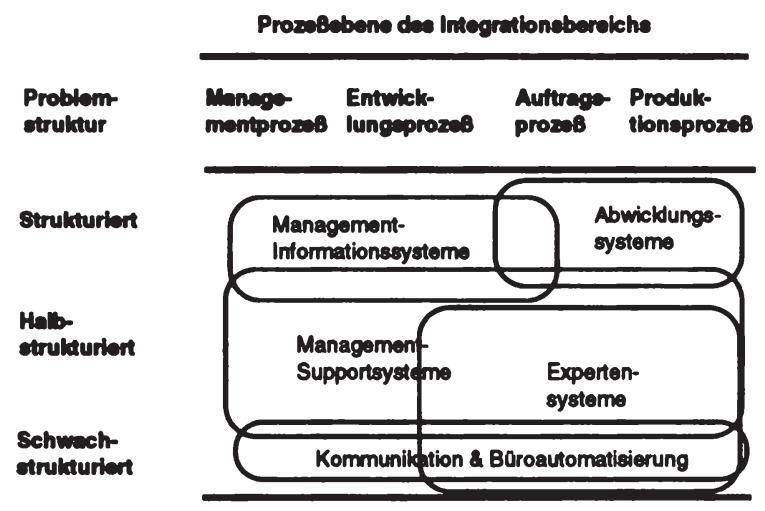

Abb. B.2.9 Klassilikation von Kommunikations- und Informationsoystemen

Sie soll als Ausgangspunkt dazu dienen, Klassen von Systemen zu unterscheiden, die unter verschiedenen situativen Bedingungen zur Integration der Unternehmung eingesetzt werden.

1 Vgl. Simon, H. A., The New Science of Management Decision, New York, New York, 1960, S. $5 \mathrm{ff}$. Simon spricht dabei von programmiert und unprogrammiert. 


\subsection{Abwicklungssysteme}

Abwicklungssysteme ${ }^{1}$ werden zur unmittelbaren Abwicklung von $\mathrm{Ge}-$ schäftsaktivitäten eingesetzt. Mit Abwicklungssystemen werden Daten, die in der Unternehmung in großen Mengen aufgrund der Abwicklung von Geschäftsaktivitäten anfallen, gesammelt, aufbereitet, eingegeben, übertragen, klassifiziert, sortiert und einer Nutzung zugänglich gemacht. ${ }^{2}$ Abwicklungssysteme sind charakteristischerweise standardisiert, detailliert, auf historische Daten ausgerichtet und ihrem Zweck entsprechend nicht auf die Analyse oder Lösung von Problemen ausgerichtet. ${ }^{3}$ Sie sind somit einsetzbar für strukturierte Probleme des Produktions- bzw. Auftragsprozesses und demnach "nordöstlich" im Klassifikationsschema einzuordnen.

Aufgrund ihrer Zuordnung zum Produktions- und Auftragsprozeß werden Abwicklungssysteme in der Literatur gelegentlich als "operative" Systeme angesehen, und von "strategischen" Systemen unterschieden, die der strategischen Planung (und damit dem Managementzyklus) zuzuordnen sind (vgl. Abb. B.2.10).

In dieser Arbeit wird demgegenüber ein (Abwicklungs-)System durchaus als "strategisch" angesehen, wenn es die Wettbewerbsposition oder deren Dauerhaftigkeit beeinflußt. "Strategisches Potential" ergibt sich durch Interdependenzen bei Gleichartigkeiten und an Schnittstellen. Kein Informationssystem und keine Informationstechnologie ist inhärent strategisch oder nicht strategisch, sondern nur deren Einsatz in der Integrationsstrategie.

1 In der Literatur liegt kein einheitlicher Begriff für diese Art von Systemen vor. Abwicklungssysteme werden auch bezeichnet als Data Processing Systems, mengenorientierte operative Systeme, Transaction Processing Systems oder Transaktionssysteme. Vgl. McLeod, R.Jr., Management Information Systems, a.a.O., S. 260; (Scheer, A.-W., EDV-orientierte Betriebswirtschaftslehre, a.a.O., S. 27; Kroeber, D.W., Watson, H.J., Computer Based Information Systems, a.a.O., 1987, S. 207; Overlack, J., Wettbewerbsvorteile durch Informationstechnologie, Frankfurt a.M. u.a.O., 1988, S. 19.

2 Vgl. z.B. Kroeber, D.W., Watson, H.J., Computer Based Information Systems, a.a.O., S. 209 ff.

3 McLeod, R.Jr., Management Information Systems, a.a.O., S. 264 ff. 
Dabei kann gerade Abwicklungssystemen eine erhebliche "strategische" Bedeutung zukommen. ${ }^{1}$
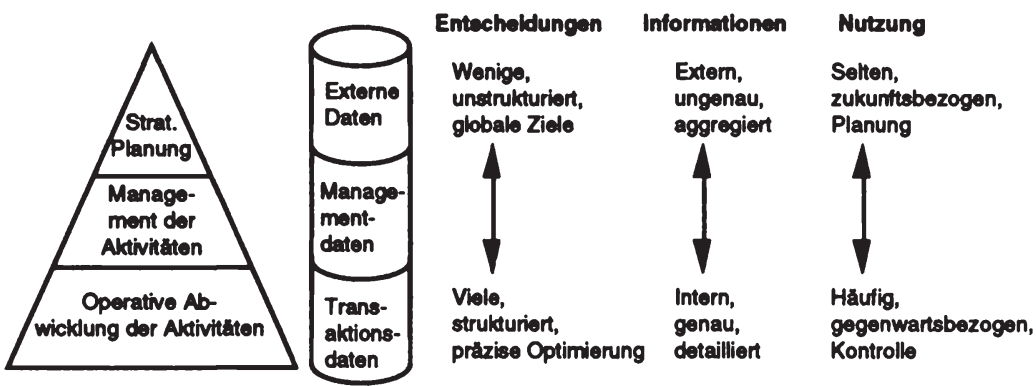

Abb. B.2.10: Traditionelle Zuordnung von Anwendungsebenen

Quelle: vgl. Kroeber, D.W., Watson, H.J., Computer Based Information Systoms, a.a.O., S. 255 - 260,

Beispiele für Abwicklungssysteme sind Bestellsysteme, Reservierungssysteme, Bankautomatisierungssysteme, Handelstransaktions- und Point-ofSale-Systeme, Verwaltungssysteme für die interne Abwicklung von Finanzbuchhaltung, Auftragsbestandsverwaltung und Personalabrechnung sowie die abwicklungsorientierten Teile von Warenwirtschaftssystemen und CAM/CAD- Systemen².

Scheer verdeutlicht am Beispiel eines Bestellprozesses, wie durch technische Integration mittels Abwicklungssystemen erheblich Zeit gespart werden kann. ${ }^{3}$ Abb. B.2.11 zeigt den Integrationsbereich Bestellprozeß mit den Geschäftsaktivitäten Disposition $D$, Lieferantenauswahl $L$ und Bestellschreibung B, zunächst ohne Abwicklungssystem. Danach benötigt die Pro-

1 Zur Charakterisierung von Informationssystemen als "strategisch" vgl. Mertens, P., Plattfaut, E., Informationstechnik als strategische Waffe, in: Information Management, Vol. 2, 1986, S. 6 ff.; Overlack, J., Wettbewerbsvorteile, a.a.O., S. 32.

2 Vgl. Scheer, A.-W., EDV-orientierte Betriebswirtschaftslehre, a.a.O., S. 209 ff.

3 Vgl. aum folgenden Scheer, A.-W., EDV-orientierte Betriebswirtschaftslehre, a.a.O., S. $26 \mathrm{ff}$. 
duktion für die Disposition 3 Stunden, für die Zustellung an den Unternehmensteil Beschaffung 8 Stunden usw.

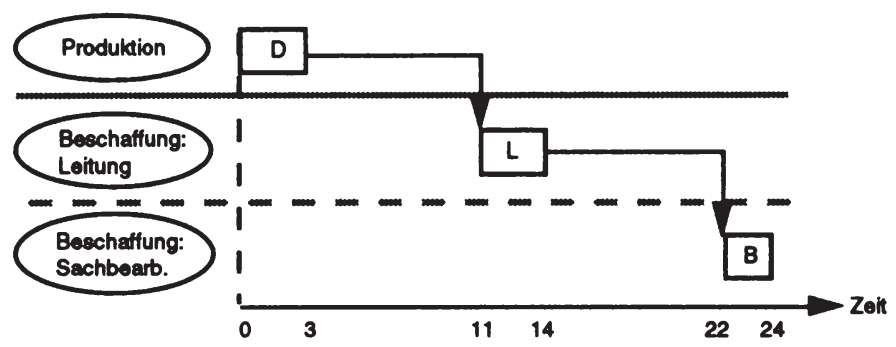

Abb. B.2.11: Ablauf eines Bestellprozesses ohne Abwicklungssystem

Durch den Einsatz eines Abwicklungssystems bei gemeinsamer Nutzung einer Datenbank kann die Zykluszeit des Bestellvorgangs auf einen Bruchteil reduziert werden (vgl. Abb. B.2.12). Die Schnittstellen sind der Ansatzpunkt zur Beschleunigung.Durch die gemeinsame Nutzung einer Datenbank ${ }^{1}$ entfallen Wartezeiten, die sich aus dem Versand zwischen den Unternehmensteilen ergeben. Weitere Verkürzungen sind möglich, wenn die Geschäftsaktivitäten D, L und B in ihre Teilschritte 1, 2, 3 zerlegt werden können. Bei Einsatz einer Datenbank muß nicht erst die Fertigstellung des Gesamtdokuments zur Weitergabe von Informationen abgewartet werden. Im Beispiel sind dabei die Reihenfolgebedingungen L2 nach D2 und B2 nach L2 einzuhalten. ${ }^{2}$

1 "A data base is a collection of data that is shared and used for multiple purposes": Kanter, J., Management Information Systems, 3rd Ed., Englewood Cliffs, New Jersey, 1984, S. 90. Die Daten sind dabei so organisiert, daß Duplikation und Redundanz vermieden werden. Informationen über Geschäftsaktivitäten werden validiert und erfaßt, um dann an einem Platz in der Datenbank gespeichert zu werden.

2 Scheer ermittelt ohne einsichtigen Grund bei gleichen Reihenfolgebedingungen eine Zykluszeit von 6 Zeiteinheiten. 


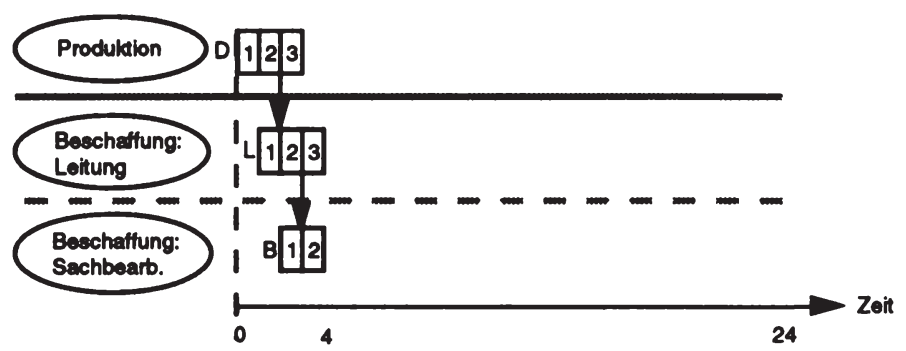

Abb. B.2.12: Ablaut cines Bestellvorganges mit gemeinsamer Datenbank

Integration mittels KIS, hier elektronische vertikale Verknüpfungen, wirken auf die Zykluszeit, wie an diesem trivialen Beispiel gezeigt werden konnte, auf zweierlei Weise. Erstens durch die Verkürzung der Übermittlungszeit von Informationen. Zweitens durch die Überlappung erst teilweise abgeschlossener Geschäftsaktivitäten. ${ }^{1}$ Die relative Bedeutung beider Faktoren wird von dem Potential der Dekomposition der Geschäftsaktivitäten und der Anzahl der Schnittstellen im Integrationsbereich abhängen. Das Prinzip der Überlappung wird für Prozesse längerer Zeitdauer, also den Entwicklungs- und den Managementzyklus, tendenziell relativ bedeutsamer sein als für den Produktions- oder Auftragszyklus.

\subsection{Management-Informationssysteme}

Management-Informationssysteme bauen auf Abwicklungssystemen auf. Sie sind nicht auf den Prozeß der Leistungserstellung selbst gerichtet, sondern eher auf dessen Steuerung und Kontrolle (vgl. Abb. B.2.13). Sie werden in der Literatur sehr unterschiedlich definiert. ${ }^{2}$ Die jüngere Literatur cha-

1 Beide Faktoren geilten nicht nur bei Abwicklungssystemen.

2 Dies geht auf eine lange Diskussion über ein Konzept für ManagementInformationssysteme (MIS) zurück. Vgl. McLeod, R.Jr., Management Information Systems, a.a.O., S. 297 ff; Kroeber, D.W., Watson, H.J., Computer Based Information Systems, a.a.O., S. 246 ff; Overlack, J., Wettbewerbsvorteile, a.a.O., 1988, S. 20; Mertens, P., Griese, J., Industrielle Datenverarbeitung II, 2. Auflage, Wiesbaden, 1979, S. 13. Die frühe Kontroverse über MIS spiegelt sich in folgenden Beiträgen wieder: Ackoff, L., (Fußnote wird auf der nächsten Seite fortgesetzt) 
rakterisiert sie nach drei Merkmalen: Funktion, Verarbeitung und ProzeB. ${ }^{1}$ Im Hinblick auf die Funktion werden dabei oft unterschieden Informationssysteme für Marketing ${ }^{2}$, Produktion ${ }^{3}$, Finanzen ${ }^{4}$ und Personal ${ }^{5}$. Das Merkmal Verarbeitung bezieht sich auf die Bereitstellung von Informationen für Managemententscheidungen. Die in ein Abwicklungssystem gelangenden Daten werden (fast) vollständig verarbeitet bzw. gespeichert und insoweit verwendet. Demgegenüber ist mit Management-Informationssystemen zwar ein Zugriff auf eine große Gesamtheit von Daten gegeben, diese werden aber nur insoweit abgerufen, wie sie für die Beantwortung einer spezifischen Frage oder zur Bearbeitung eines spezifischen Problems von Bedeutung sind. 6 Abwicklungssysteme stellen jedoch die dazu erforderliche Basis bereit, nämlich die Grundgesamtheit abrufbarer Daten ${ }^{7}$ (vgl. Abb. B.2.13).

Misinformation Systems, a.a.O., S. B147 - B156; Rappaport, A., Management Misinformation Systems, - Another Perspective, in: Management Science, Vol. 15, S. B 133 - B 136; Dearden, J., MIS Is a Mirage, in: Harvard Business Review, Vol. 50, JanuaryFebruary, S. 90 - 99.

1 Vgl. z.B. Kroeber, D.W., Watson, H.J., Computer Based Information Systems, a.a.O., S. 252.

2 Vgl. McLeod, R.Jr., Management Information Systems, a.a.O., S. 491 ff; Meffert, H., Informationssysteme, a.a.O.; Kotler, Ph., A Design for the Firm's Marketing Nerve Center, in: Business Horizons, Vol. 9, 1966, S. 70.

3 Vgl. Cox, J.F., Clark, St.J., Problems in Implementing and Operating a Manufacturing Resource Planning Information System, Journal of Management Information Systems, Vol. 1, Summer 1984, S. 81 - 101; McLeod, R. Jr., Management Information Systems, a.a.O., S. $533 \mathrm{ff}$.

4 Vgl. McLeod, R. Jr., Management Information Systems, a.a.O., S. 575 ff; Gershefski, G.W., Building a Corporate Financial Model, in: Harvard Business Review, Vol. 47, July-August 1969, S. 61 - 72; Gentry, J.A., State of the Art of Short-Run Financial Management, in: Management, Vol. 17, Summer 1988, S. 41 - 57.

5 Vgl. DeSanctis, G., Human Resource Information Systems: A Current Assessment, in: MIS Quarterly, Vol. 10, March 1986, S. 15 - 26.; Heinrich, L.J., Pils, M., Betriebsinformatik im Personalbereich - Die Planung computengestützter Personalinformationssysteme, 2. Auflage, Würzburg, Wien, 1983; Kroeber, D.W., Watson, H.J., Computer Based Information Systems, a.a.O., S. 301 - 326.

6 Insofern sind Daten der Gegenstand von Abwicklungssystemen. Demgegenüber sind Informationen als problemlösungsbezogene Daten Gegenstand von ManagementInformationssystemen. Vgl. z.B. Overlack, J., Wettbewerbsvorteile, a.a.O., S. 18 und dort angegebene Literatur.

7 Vgl. Kroeber, D.W., Watson, H.J., Computer Based Information Systems, a.a.O., S. 216. 


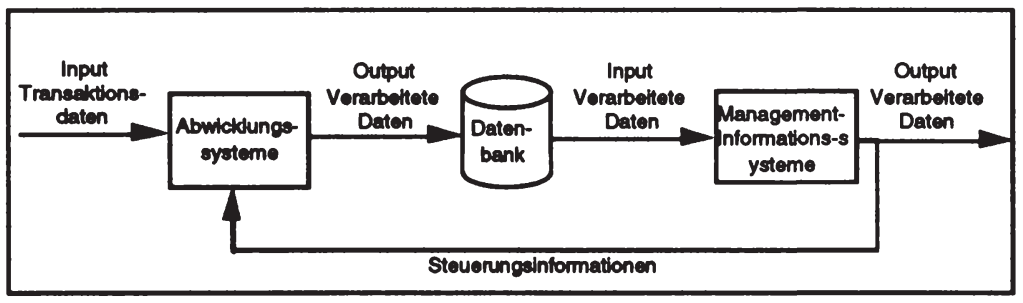

Abb. B.2.13: Serielle Bezichung zwischen Abwicklungssystem und Managementinformationssystem

Quelle: Kroeber, D.W., Watson, H.J., Computer Based Information Systems, a.a.O., S. 217

Das Merkmal Prozeß bezieht sich auf die Ebene des Geschäftsprozesses. Während Abwicklungssysteme unmittelbar auf die Leistungserstellung gerichtet sind, übernehmen Management-Informationssysteme deren Steuerung auf der übergeordneten Prozeßebene. Daraus ergibt sich im eingangs eingeführten Klassifikationsschema eine Einordnung im "nordwestlichen" Bereich.

Möglichkeiten zur Integration von Unternehmensteilen auf Basis von Management-Informationssystemen bieten sich entlang dieser drei Merkmale. In Bezug auf den Prozeß kommen integrative Verbindungen der verschiedenen Prozeßebenen (Produktionsprozeß bis Managementprozeß) in Betracht. Ein Beispiel ist die Analyse von regionalen Abverkaufsdaten von Konsumartikeln (Auftragsprozeß) mit verschiedener regionaler Kommunikations- oder Preispolitik (Managementprozeß). So kann die Wirkung einer regional geschalteten Werbung anhand der realen Abverkaufszahlen der Points of Sale ermittelt werden; aufwendigere herkömmliche Marktforschungsmethoden können zum Teil entfallen. Hinsichtlich der Verarbeitung erfolgt Integration durch die Verknüpfung von Abwicklungs- und Management-Informationssystemen. Die funktionale Integration wird durch die gemeinsame Nutzung einer Datenbank durch verschiedene Unternehmensteile unterstützt. Eine Information wird in einem Unternehmensteil gewonnen und nur an einer einzigen Stelle gespeichert, ist jedoch von anderen Unternehmensteilen nutz- und änderbar. Dies entspricht der ökonomischen Grundlogik horizontaler Verflechtung. Gemeinsam genutzte Daten bilden eine Gleichartigkeit. An diesem Beispiel wird auch ein 
Problem der Integration deutlich. So wirkt sich eine fehlerhafte Eingabe von Daten in einem Unternehmensteil auf alle anderen Nutzer aus. Zudem werden verschiedene Unternehmensteile verschiedene oder verschieden genaue Informationen über eine Geschäftsaktivität benötigen. Beispielsweise sieht der Marketingbereich ein Angebot, aus dem mit hoher Wahrscheinlichkeit bereits ein Auftrag hervorgeht, bereits als "harte" Information an, während dieselbe Information für die Produktion nur vorläufige Aussagen enthält, die noch abzusichern und zu präzisieren sind. Breiter Zugang zu Daten wirft zudem Probleme in der Datensicherheit auf. Festzuhalten ist für die weitere Betrachtung, daß ein zu hoher Integrationsgrad negativen Integrationsnutzen verursachen kann.

\subsubsection{3}

\section{Management-Unterstützungssysteme}

Management-Unterstützungssysteme unterstützen die Entscheidungsfindung bei semi-strukturierten Entscheidungsproblemen. ${ }^{1}$ Dabei wird unterschieden zwischen Entscheidungs-Unterstützungssystemen (Decision Support Systems, DSS), Gruppenentscheidungs-Unterstützungssystemen (Group Decision Support Systems, GDSS) und Executive-Unterstützungssystemen (Executive Support Systems, ESS). Bei semi-strukturierten Problemen (vgl. Abb. B.2.14) können im Rahmen des KIS von den Management-Unterstützungssystemen noch die Expertensysteme unterschieden werden (vgl. dazu Abschnitt 2.3.1.1.4).

1 Vgl. zum folgenden Rockart, J.F., DeLong, D., Executive Support Systems, a.a.O., S. 14 ff; DeSanctis, G., Gallupe, R.B., Group Decision Support Systems: A New Frontier, Data Base, Winter 1985, S. 3; Keen, P.G.W., Scott Morton, M.S., Decision Support Systems, a.a.O, S. 1; Kroeber, D.W., Watson, H.J., Computer Based Information Systems, a.a.O., S. 376; Vogel D., Nunamaker J., Applegate L., Konsynski B., Group Decision Support Systems: Determinants of Success, DSS-87 Transactions, 7th International Conference an Decision Support Systems, 1987, S. 118 - 128. 


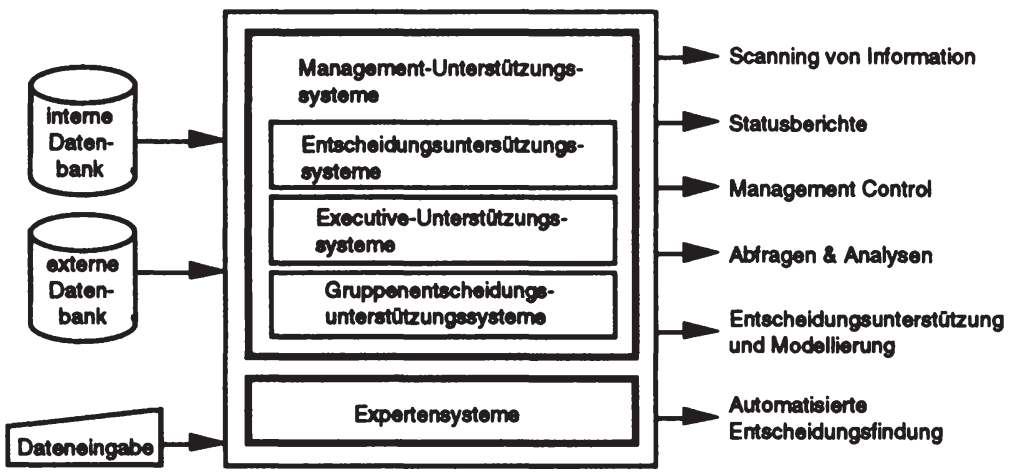

Abb. B 2.14: Ūbersicht Uber KIS bei semi-strukturierter Problemstruktur

Die Beschreibung des semi-strukturierten Bereichs von Entscheidungsproblemen folgt in der Literatur häufig Simons Definition dreier Phasen eines Problemlösungs- oder Entscheidungsprozesses: die Problemaufdeckung, die Alternativensuche und die Alternativenauswahl (Intelligence1, Design, Choice)2.

Als völlig strukturiert gilt ein Entscheidungsproblem, das im Hinblick auf alle drei Phasen strukturiert ist, d.h. es können Regeln angegeben werden, nach denen alle relevanten Entscheidungsprobleme aufgedeckt, Handlungsalternativen ermittelt und daraus eine Handlungsalternative ausgewählt werden kann. Wenn keine Regeln zur Problemaufdeckung, Alternativensuche und -auswahl angegeben werden können, ist das Entscheidungsproblem unstrukturiert.

1 Der Begriff Intelligence hat dabei nichts mit dem deutschen Begriff Intelligenz au tun und wird daher als "Problemaufdeckung" übersetzt: "The first phase of the decisionmaking process - searching the environment for conditions calling for decision - I shall call intelligence activity (borrowing the military meaning of intelligence)": Simon, H.A., Management Decision, a.a.O., S. 2.

2 Vgl. Simon, H.A., Management Decision, a.a.O., S. 2 ff; Keen, P.G.W., Scott Morton, M.S., Decision Support Systems, a.a.O, S. 95 ff; danach McLeod, R. Jr., Management Information Systems, a.a.O., S. 333 ff; Kroeber, D.W., Watson, H.J., Computer Based Information Systems, a.a.O., S.379. Dabei umfaßt die Problemaufdeckung die Suche nach Entscheidungsproblemen in der Umwelt, die Alternativensuche das Erfinden und Entwickeln von Handlungsalternativen und die Alternativenauswahl die Auswahl einer (Fußnote wird auf der nächsten Seite fortgesetzt) 
Semi-strukturierte Probleme treten auf, wenn einzelne Phasen strukturiert sind, andere aber nicht. Beispielsweise sind viele Entscheidungsprobleme des Investment Managements durch Portfolio-Selection-Modelle, Optionspreismodelle oder Lineare Programmierungsmodelle theoretisch abbildbar und insoweit strukturiert. Darin sind jedoch sehr wesentliche "weiche" Informationen nicht berücksichtigt, beispielsweise das Gerücht der Illiquidität einer Bank oder die Einschätzung der "richtigen" Volatilitätskennziffer. Insofern mag die Alternativensuche und die Vorbereitung der Alternativenauswahl durchaus computergestützt erfolgen, in einigen Fällen ist jedoch die Anpassung durch menschliche Entscheidungsträger notwendig. Dabei erlauben immer benutzerfreundlichere Technologien, insbesondere im Bereich der künstlichen Intelligenz, Probleme, die zuvor für unstrukturiert gehalten wurden, zumindest teilweise zu strukturieren

\subsection{Entscheidungs-Unterstützungssysteme}

Ein Entscheidungs-Unterstützungssystem macht Entscheidungsmodelle und entscheidungsrelevante Informationen zugänglich und erlaubt die Verknüpfung der beiden. ${ }^{1}$ Entscheidungs-Unterstützungssysteme werden häufig interaktiv genutzt, und zwar von Entscheidungsträgern ohne (umfassende) Computererfahrung. ${ }^{2}$ Sie sind auf semi-strukturierte Entscheidungsprobleme gerichtet ${ }^{3}$ und flexibel an die Problemstellung, den Nutzer und Umweltänderungen anpaßbar.4 Auf Basis einer empirischen Analyse wurden sechs Funktionen von Entscheidungs-Unterstützungssy-

der möglichen Handlungsalternativen.

1 Kroeber, D.W., Watson, H.J., Computer Based Information Systems, a.a.O., S. 376, Keen, P.G.W., Value Analysis: Justifying Decision Support Systems, in: MIS Quarterly, Vol. 5, March 1981, S. 1; King, W.R., Decision Support Systems, a.a.O., S. 423; McLeod, R. Jr., Management Information Systems, a.a.O., S. 340.

2 Vgl. Keen, P.G.W., Value Analysis, a.a.O., S. 1.

3 Vgl. Keen, P.G.W., Scott Morton, M.S., Decision Support Systems, a.a.O, S. 1.

4 Vgl. Sprague, R.H., Carison, E.D., Building Effective Decision Support Systems, Englewood Cliffs, New Jersey, 1982, S. 4. 
stemen unterschieden. Diese sind, nach wachsendem Unterstützungs- und Komplexitätsgrad geordnet: die Abfrage einzelner Informationen, die Analyse ganzer Vorgänge, die Vorbereitung von Berichten aus mehreren Vorgängen, die Abschätzung von Entscheidungskonsequenzen, das Vorschlagen von Entscheidungen und das Treffen von Entscheidungen. ${ }^{1}$ Gegenüber den Management-Informationssystemen sind Entscheidungs-Unterstützungssysteme schnell und flexibel für ad hoc sich ergebende Fragen einsetzbar, sie erlauben den Einsatz spezifischer Sensitivitätsanalysen und von Optimierungs- oder Simulationsmodellen ${ }^{2}$ und können benutzerfreundlich von Nicht-Computerfachleuten (weiter-)entwickelt werden. ${ }^{3}$

King definiert drei Kriterien, die einem Entscheidungs-Unterstützungssystem "strategische" Bedeutung geben. ${ }^{4}$ Erstens muß es auf ein Ziel oder eine Klasse von Problemen gerichtet sein, die normalerweise als "strategisch" zu bezeichnen sind im Hinblick auf Umfang, Wichtigkeit, Zeithorizont und die Bindungswirkung bzw. Irreversibilität des Entscheidungsproblems. Zweitens muß mit der Verarbeitung von Informationen durch das Entscheidungs-Unterstützungssystem ein Wettbewerbsvorteil angestrebt werden. Drittens muß das Entscheidungs-Unterstützungssystem direkt auf die Kernfaktoren der betrachteten strategischen Entscheidungen wirken, etwa durch den Abbau von Unstrukturiertheit. Diese Kriterien erscheinen als verwandt mit der in dieser Arbeit vorgelegten Sichtweise. EntscheidungsUnterstützungssysteme sind, wie auch andere KIS, nicht per se "strategisch", sondern nur, wenn sie in einem Integrationsbereich zur Verbesserung der Wettbewerbsposition oder ihrer Dauerhaftigkeit beitragen.

1 Vgl. Alter , St.,L. How Effective Managers Use Information Systems, in: Harvard Business Review, Vol. 54, November-December 1976, S. 97 - 104.

2 Einen guten Überblick gibt McLeod, R. Jr., Management Information Systems, a.a.O., S. 344 ff.

3 Die Unterscheidung von Management-Informationssystemen und Entscheidungs-Unterstützungssystemen ist konzeptionell und nicht technologisch bestimmt. Vergleiche finden sich bei Keen, P.G.W., Wagner, G.R., DSS: An Executive Mind-Support System, in: Datamation, November, 1979, S. 117 ff; Keen, P.G.W., Scott Morton, M.S., Decision Support Systems, a.a.O, S. 1 f; Kroeber, D.W., Watson, H.J., Computer Based Information Systems, a.a.O., S. 381 ff.

4 Vgl. King, W.R., Decision Support Systems, a.a.O., S. 429. 


\subsection{Gruppenentscheidungs-Unterstützungssysteme}

Gruppenentscheidungs-Unterstützungssysteme sind KIS, die Gruppen dabei unterstützen sollen, Entscheidungsprobleme zu identifizieren, zu formulieren und zu lösen. ${ }^{1}$ Sie gleichen damit prinzipiell den allgemeinen Entscheidungs-Unterstützungssystemen, stellen jedoch im Vergleich zu jenen besonders die Förderung des Ideen- und Meinungsaustauschs und die Diskussion von Zielen und Prämissen in den Vordergrund.2

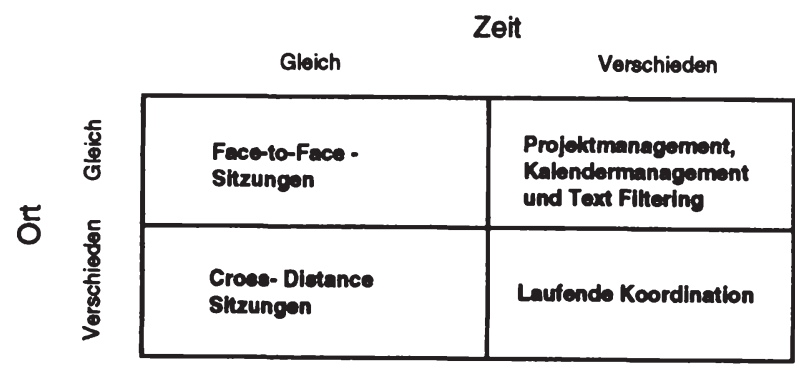

Abb. B.2.15: Matrix von Gruppenentscheidungs-Unterstützungssystemen Quelle: Bullen, Ch.V., Johansen, R., Groupware, a.a.O., S. 21

1 Eine Auswahl der Fülle von Artikeln und Monographien zu diesem auch als "Computer Support of Cooperative Work" (CSCW) bezeichneten Feld ist Bullen, Ch.V., Johansen, R., Groupware: A Key to Managing Business Teams, Working Paper No. 138, Massachusetts Institute of Technology, Center for Information Systems Research, Cambridge, Massachusetts, 1988; Johansen, R., Groupware Computer Support for Business Teams, New York, New York, 1988, , Johansen, R., Teleconferencing and Beyond, New York, New York, 1984; Lowe, D., Cooperative Structuring of Information: The Representation of Reasoning and Debate, Journal of Man-Machine Studies, Vol. 23, 1985, S. 97 - 111; , Huber, G.P., Issues in the design of group decision support systems, MIS Quarterly, Vol. 8, 1984; Huber, G.P., A Theory of the Effects of Advanced Information Technologies on Organizational Design, Intelligence, and Decision Making, Academy of Management Review, Vol. 15, 1990, S. 47 71.

2 Vgl. Gallupe, R.R., DeSanctis, G., Computer-Based Support for Group Problem-Finding: An Experimental Investigation, in: MIS Quarterly, Vol. 12, June 1988, S. 287. 
In Abb. B.2.15 sind verschiedene Einsatzmöglichkeiten von Gruppenentscheidungs-Unterstützungssystemen nach ihrem Orts- und Zeitbezug systematisiert. 1

\subsection{Executive-Unterstuitzungssysteme}

Executive-Unterstützungssysteme ${ }^{2}$ unterscheiden sich von EntscheidungsUnterstützungssystemen durch die Gruppe der Nutzer, nämlich das TopManagement, durch breiter angelegte, stärker auf aggregrierte und externe Informationen ausgerichtete Anwendungen, durch stärkere Kommunikations- als Analyseorientierung und durch Implementierungsbeteiligung des Top-Managements. ${ }^{3}$

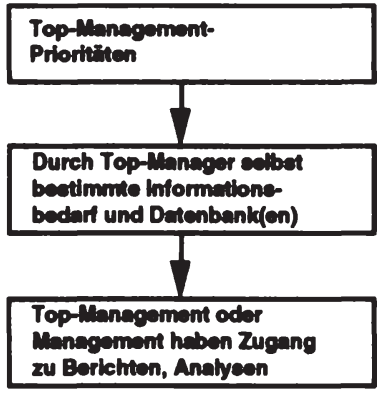

Abb. B.2.16: Wichtigste Schritte boi der Definition eines ESS

Quello: Rockart, J. F., DeLong, D., Executive Support Systems, a.a.O., S. 27

1 Vgl. Bullen, Ch.V., Johansen, R., Groupware, a.a.O., S. 21; vgl. auch DeSanctis, G., Gallupe, R.B., A Foundation for the Study of Group Decision Support Systems, in: Management Science, Vol. 33, 1987, wo nach Ort und Gruppengröße differenziert wird. Auf eine Diskussion der einzelnen Elemente der Felder wird hier verzichtet. Vgl. dazu Bullen, Ch.V., Johansen, R., Groupware, a.a.O., S. 1 ff.

2 Entsprechend dem Begriff der Management-Unterstützungssysteme wird der Begriff Executive nicht übersetzt, da die Alternativbegriffe unpassend ("Exekutiv") oder ebenfalls englisch ("Top-Manager") sind.

3 Vgl. Rockart, J. F., DeLong, D., Executive Support Systems, a.a.O., S. 21 ff. 
Als von zentraler Bedeutung zur Gestaltung des ExecutiveUnterstützungssystems erscheint die Definition des Informationsbedarfs durch das Top-Management selbst (vgl. Abb. B.2.16). Nach einer empirischen Untersuchung, werden drei Funktionen und drei Zwecke von Executive-Unterstützungssystemen unterschieden. ${ }^{1}$ Die Funktionen sind die Kommunikation zwischen Top-Managern durch elektronische Netze, die Möglichkeit der menugesteuerten Abfrage von Informationen aller Art sowie (seltener) die Analyse und Modellbildung. Als Zwecke von Executive-Unterstützungssystemen wurden gefunden die Steigerung von Effizienz und Effektivität des Top-Managements, die Unterstützung von Planungs- und Kontrollaktivitäten sowie die Verbesserung des "mentalen Modells", das ein Top-Manager von seinem Unternehmen hat. ${ }^{2}$

\subsection{Expertensysteme}

Künstliche Intelligenz kann definiert werden als der Versuch, menschliche Fähigkeiten maschinell nachzubilden, wie etwa das Sehen, Hören oder Denken. Expertensysteme sind ein Teilbereich der künstlichen Intelligenz. Mit ihnen wird versucht, besonders schwierige und komplexe Probleme auf dem Fähigkeitenniveau eines Experten zu lösen. Ein Experte kann schwierige Probleme lösen, das Ergebnis erklären, lernen, Wissen ordnen, Relevanz bestimmen, und er weiß, was er nicht weiß. Expertensysteme sind Computerprogramme, die das Verhalten eines Experten nachstellen, wobei

1 Vgl. Rockart, J. F., DeLong, D., Executive Support Systems, a.a.O., S. 26 ff.

2 Weitere empirische Studien legen vor: Rockart, J.F., Treacy, M.E., The CEO goes On-Line, in : Harvard Business Review, January-February 1982, S. 82 - 88; Brady, R.H., Computer in Top-Level Decision Making, in: Harvard Business Review, Vol. 45, July-August 1967, S. 67 - 76; Jones, J.W., McLeod, R., The Structure of Executive Information Systems: An Exploratory Analysis, in: Decision Sciences, Vol. 17, Spring 1986, S. 220 - 249. Einen Überblick gibt McLeod, R. Jr., Management Information Systems, a.a.O., S. 455 ff. 
sich die heutige Technologie (noch) besonders auf die Problemlösung und Lösungserklärung beschränkt. 1

Im Gegensatz zu Entscheidungs-Unterstützungssystemen wirken Expertensysteme nicht nur problemlösungsunterstützend, sondern bieten auch Lösungen an, d.h. treffen Entscheidungen. Expertensysteme haben vielfältige betriebliche Anwendungen gefunden. Im Bereich Produktionsplanungund Steuerung (PPS) sind dies z. B. Analyse- und Diagnosesysteme, Selektionssysteme, Intelligente Checklisten, Beratungssysteme, Konfigurierungssysteme, Planungssysteme, Zugangssysteme, Hilfesysteme, Lehr- und Unterrichtssysteme, Entscheidungssysteme und Überwachungssysteme. ${ }^{2}$

Zur Identifikation von Entscheidungsproblemen, für die der Einsatz von Expertensystemen geeignet erscheint, werden in der Literatur drei Kriterien genannt. Danach ist der Einsatz eines Expertensystems für eine Aufgabe bzw. Geschäftsaktivität geeignet, falls diese verbessert würde, erstens stünde mehr Zeit zur Entscheidungsfindung zur Verfügung, könnte zweitens der beste Experte sich der Aufgabe jeweils widmen und schließlich konsistenter entschieden werden. ${ }^{3}$

Die Logik von Expertensystemen ist eine immaterielle horizontale Verflechtung: die breitere Verteilung von knappem, gleichartigem Expertenwissen auf mehr Unternehmensteile. Auf die Integration können große, unternehmensweit eingesetzte Expertensysteme wirken, wenn sie eine möglichst breite, gleiche Anwendung in allen Unternehmensteilen ermöglichen. Dem sind jedoch Grenzen gesetzt, da generell von einem Tradeoff

1 Vgl. Leonard-Barton, D., Sviokla, J.J., Putting Expert Systems to Work, in: Harvard Business Review, Vol. 66, 1988, S.93. Ein Überblick über die Funktionsweise von Expertensystemen findet sich z.B. bei McLeod, R.Jr., Management Information Systems, a.a.O., S. 412 ff; Scheer, A.-W., EDV-orientierte Betriebswirtschaftslehre, a.a.O., S. 171 ff.

2 Scheer, A.-W., EDV-orientierte Betriebswirtschaftslehre, a.a.O., S. 183 ff.

3 Leonard-Barton, D., Sviokla, J.J., Expert Systems, a.a.O., S. 94. 
zwischen der Breite der Einsetzbarkeit und dem Niveau der Expertise eines Expertensystems auszugehen ist (vgl. Abb. B.2.17).

Zudem finden sich in der Praxis auch davon völlig verschiedene Ansätze. So folgt DuPont einem "Bottom-Up"-Ansatz: Zwar sind Expertensysteme in der Unternehmung so verbreitet wie Tabellenkalkulationsprogramme, werden aber lediglich lokal bzw. dezentral eingesetzt und nur mit dem Anspruch, Laien auf Fachleute-Niveau anzuheben. ${ }^{1}$

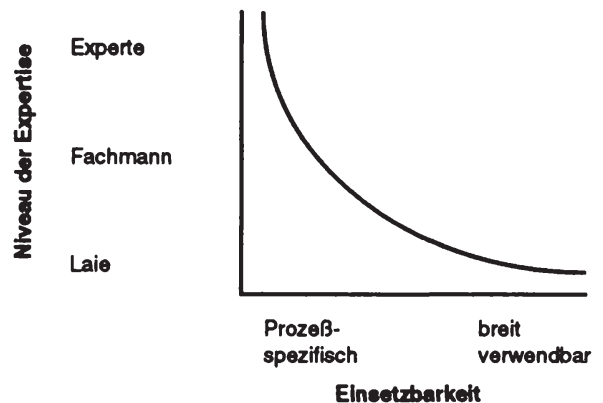

Abb. B.2.17: Tradeoff zwischen Niveau und Einsetzbarkeit von Expertensystemen

Quelle: In Anlehnung an ein persőnliches Gesprăch mit John Sviokla am 23. Januar 1990 in Boston, Mass.

Expertensysteme wurden in den "südöstlichen" Bereich der Klassifikation in Abb. B.2.9 eingeordnet. Von den Systemen bei semi-strukturierter Problemstruktur dringen Expertensysteme am weitesten in das Feld der schwachstrukturierten Probleme vor. Ihr Einsatz ist jedoch etwa im Vergleich zu Executive-Unterstützungssystemen (noch) überwiegend auf den Produktions- und Auftragsprozeß begrenzt.

1 Vgl. Keil, M., Sviokla, J.J., Du Pont's AI Task Force: The Water Intrusion Expert System, Boston, Massachusetts, Harvard Business School Case No. 9-189-189, 1989, S. 1. 


\subsection{Btiroautomatisierungssysteme}

Die Büroautomatisierung ist die multifunktionale, integrierte, computergestützte Verarbeitung und Kommunikation von Daten, Texten, Bildern und Sprache. ${ }^{1}$ Büroautomatisierungssysteme umfassen dabei weit mehr als die klassischen Sekretariatsfunktionen und reichen weit in das Tätigkeitsgebiet qualifizierter Sachbearbeiter hinein. ${ }^{2}$ Die Büroautomatisierung umfaßt die Textverarbeitung, Electronic Mail, Voice Mail, Elektronische Kalenderverwaltung, Audiokonferenzen, Videokonferenzen, Computerkonferenzen ${ }^{3}$, Telefax, die Bild- und Graphikbearbeitung und -speicherung sowie das Desktop Publishing. 4

Büroautomatisierungssysteme strukturieren den Inhalt der durch sie ausgeführten Kommunikation kaum, im Gegensatz etwa zu Modellen bei Entscheidungs-Unterstützungssystemen, und erlauben insofern jegliche Darstellung auch schwachstrukturierter Probleme. Sie wurden daher dem "südlichen" Bereich des Klassifikationsschemas (Abb. B.2.9) zugeordnet. Der Einsatz von Büroautomatisierungssystemen kann für die Integration der Unternehmung von hoher Bedeutung sein, da sie viele Prozesse bzw. Zyklen der Unternehmung beschleunigen. 5 Ursache hierfür sind vor allem die sich stets verbessernden Kommunikationsmöglichkeiten zwischen den

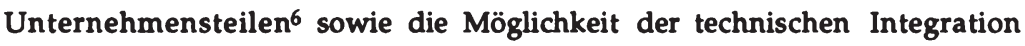
(vertikale Verknüpfung) von Büroautomatisierungssystemen mit anderen Kommunikations- und Informationssystemen.7

1 Vgl. z.B. Kroeber, D.W., Watson, H.J., Computer Based Information Systems, a.a.O., S. 331; Scheer, A.-W., EDV-orientierte Betriebswirtschaftslehre, a.a.O., S. 260.

2 Vgl. McLeod, R.Jr., Management Information Systems, a.a.O., S. 378.

3 Vgl. Chess, D.M., Colishaw, M.F., Conferencing, in: IBM Systems Journal, Vol. 26, No. 1, 1987, S. 138.

4 Nähere Beschreibungen finden sich bei McLeod, R. Jr., Management Information Systems, a.a.O., S. 379 ff; Scheer, A.-W., EDV-orientierte Betriebswirtschaftslehre, a.a.O., S. 260 ff.

5 Picot weist auf die vielfältigen Integrationspotentiale der Kommunikationstechnik selbst hin. Vgl. Picot, A., Kommunikationstechnik, a.a.O., S. 379 ff.

6 Vgl. McLeod, R.Jr., Management Information Systems, a.a.O., S. 400 ff.

7 Vgl. Kroeber, D.W., Watson, H.J., Computer Based Information Systems, a.a.O., S. 358. 


\subsubsection{Koordinationsmechanismen}

Koordinationsmechanismen werden zur organisationalen Bildung vertikaler Verknüpfungen und horizontaler Verflechtungen im Integrationsbereich eingesetzt. Koordination ist eine Grunddimension der Organisationsstruktur. ${ }^{1}$ So steht Koordination im fünfdimensionalen System zur Beschreibung der Organisationsstruktur von Kieser und Kubicek neben Spezialisierung, Konfiguration, Entscheidungsdelegation und Formalisierung. ${ }^{2}$ Die Koordinationsmechanismen sind die einzelnen Instrumente, die zur Koordination der Unternehmung eingesetzt werden. Koordination wurde von Integration unterschieden. Die Koordination umfaßt die eigentliche Abstimmung im Rahmen bestehender Koordinationsmechanismen. ${ }^{3}$ Gegenstand der (organisationalen) Integration ist es hingegen, zweckmäßige Arten von Koordinationsmechanismen zu bestimmen.

Die Koordinationsmechanismen werden in der Literatur sehr verschieden klassifiziert. So unterscheiden March und Simon die Koordination durch Planung von der Koordination durch Feedback.4 Jener grundsätzlichen Klassifikation sind viele weitere gefolgt, die teilweise spezifischer einzelne Koordinationsmechanismen angeben und/oder ordnen (vgl. Abb. B.2.18) ${ }^{5}$.

1 Vgl. Grochla, E., Einführung in die Organisationstheorie, Stuttgart, 1978, S. 31 - 32; Kieser, A., Kubicek, H., Organisation, a.a.O., S.71 - 80; Welge, M.K., Unternehmensführung, Band 2: Organisation; unter Mitwirkung von Herbert Kubicek, Stuttgart, 1986, S. 393.

2 Vgl. Kieser, A., Kubicek, H., Organisation, a.a.O., S. 79.

3 Vgl. Abschnitt A.2.2 sowie Kosiol, E., Organisation, a.a.O., S. 172; Mellerowicz, K., Unternehmenspolitik, a.a.O., S. 243; Lawrence, P., Lorsch, J., Organization and Environment, a.a.O., S.11; Heinen, E., Industriebetriebslehre, a.a.O., S.25.

4 Vgl. March, J.E., Simon, H.A., Organizations, a.a.O., S. 160.

5 Ähnliche Übersichten, die auch andere Ansätze umfassen, finden sich bei Welge, M.K., Unternehmensführung, Bd. 2: Organisation, unter Mitwirkung von Herbert Kubicek, Stuttgart, 1986, S. 413; Benkenstein, M., F\&E und Marketing - Eine Untersuchung der Leistungsfähigkeit von Koordinationskonzepten bei Innovationsentscheidungen, Wiesbaden, 1987, S. 135. 


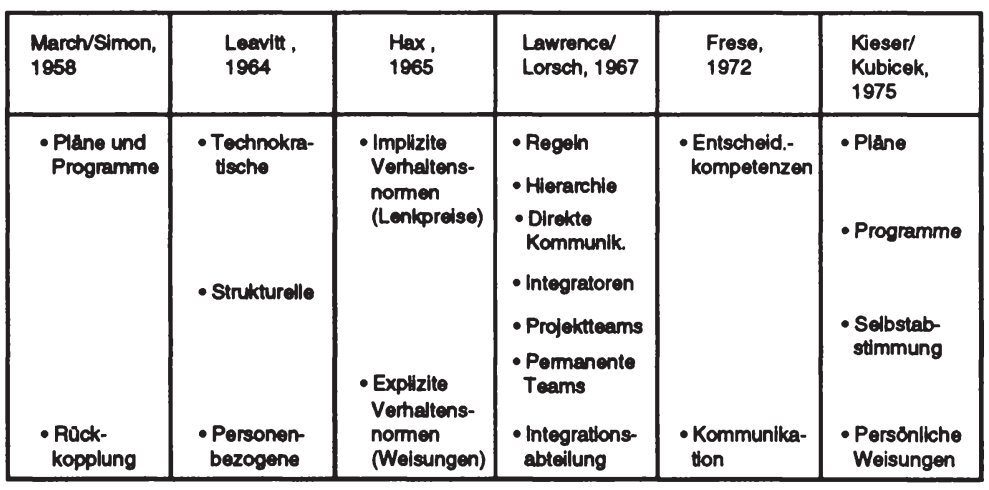

\begin{tabular}{|c|c|c|c|c|c|}
\hline $\begin{array}{l}\text { Van de Ven } \\
\text { Delbeco/Koenig. } \\
1976\end{array}$ & $\begin{array}{l}\text { Khandwalla, } \\
1977\end{array}$ & z, & $\begin{array}{l}\text { Galbralth } \\
\text { Kazanlian, } \\
1986\end{array}$ & $\begin{array}{l}\text { Tushman } \\
\text { Nadler, } \\
1989\end{array}$ & \\
\hline $\begin{array}{c}\text { - Persónlich } \\
\text { Hierarchle } \\
\text { Lateral } \\
\text { - Teambildung } \\
\text { Geplant } \\
\text { Ungeplant }\end{array}$ & $\begin{array}{l}\text { - Hierarchle } \\
\text { - Stendard- } \\
\text { sterung } \\
\text { - Regeln } \\
\text { - Verbindungs- } \\
\text { stellen } \\
\text { - Teams und } \\
\text { partizipattve } \\
\text { Entscheld.- } \\
\text { findung } \\
\text { - Verhaltens- } \\
\text { wiss. Meth. } \\
\text { - Ausbildung }\end{array}$ & $\begin{array}{l}\text { - Verbindungs- } \\
\text { elnrichtungen } \\
\text { (nach Galbralth } \\
\text { [1973] S. 48) }\end{array}$ & $\begin{array}{l}\text { - Hierarchie } \\
\text { - Regeln } \\
\text { - Zlelvorgaben } \\
\text { - Direkte } \\
\text { Kommunlk. } \\
\text { - Verbindungs- } \\
\text { stellen } \\
\text { - Projoktteams } \\
\text { - Integratoren } \\
\text { - Integrations- } \\
\text { abtellung }\end{array}$ & $\begin{array}{l}\text { - Management } \\
\text { Prozesse } \\
\text { (Anrelz- und } \\
\text { Kontrollsyst.) } \\
\text { - Hierarchie } \\
\text { - Strukturelle } \\
\text { Verbindungen } \\
\text { Verb.stelle } \\
\text { Cross-Unit- } \\
\text { Group } \\
\text { Integrator } \\
\text { Integrations- } \\
\text { abteilung } \\
\text { Matrixorg. }\end{array}$ & $\begin{array}{l}\text { - Administrative } \\
\text { Planung } \\
\text { Programme } \\
\text { - Strukturelle } \\
\text { Hierarchie } \\
\text { Querver- } \\
\text { bindungen } \\
\text { - Personale } \\
\text { (In)formale } \\
\text { Teams } \\
\text { Ausbildung, } \\
\text { Kultur }\end{array}$ \\
\hline
\end{tabular}

Abb. B.2.18: Ausgewahlte Kassifikationen von Koordinationsmechanismen

Die Unterschiede in der Bildung von Kontinua von Koordinationsmechanismen ergeben sich dabei aus den unterschiedlichen Erklärungszielen und Gliederungskriterien sowie der unterschiedlichen Systematik des jeweiligen Bezugsrahmens der genannten Autoren. So geben Kieser und Kubicek ihr Gliederungskriterium als das jeweils institutionalisierte Koordinationsmedium an, ${ }^{1}$ Schanz die Art der Abstimmung (wechselseitig im

1 Kieser, A., Kubicek, H., Organisation, a.a.O., 1983, S.112. Als weiteres mögliches Kriterium nennen sie den Zeitpunkt der Koordination (Vorauskoordination vs. Feedbackkoordination). Dies., Organisation, a.a.O., 1983, S. 109. Dieses Kriterium wird jedoch selten tatsächlich verwendet. Vgl. Benkenstein, M., F \& E und Marketing Eine Untersuchung der Leistungsfähigkeit von Koordinationskonzeptionen bei (Fußnote wird auf der nächsten Seite fortgesetzt) 
Vergleich zur Standardisierung) ${ }^{1}$, und Frese unterscheidet nach der Art der Koordination selbst (Kompetenzverteilung im Vergleich zum Informationsaustausch). ${ }^{2}$ In der eher empirisch orientierten amerikanischen Literatur wird als Gliederungskriterium stärker der Charakter der zu koordinierenden Aufgabe herangezogen. So verwenden March und Simon als Kriterium die Stabilität und Vorhersehbarkeit der zu koordinierenden Situation. ${ }^{3}$ Khandwalla nennt die Umweltkomplexität und -heterogenität, ${ }^{4}$ und Van de Ven, Delbeqc und Koenig nennen die Unsicherheit der betrachteten Aufgabe (Task uncertainty) als wichtigstes, auch empirisch bestätigtes Unterscheidungskriterium. ${ }^{5}$ Aufbauend auf den empirischen Ergebnissen von Lawrence und Lorsch, ordnen auch Galbraith und Kazanjian die Koordinationsmechanismen nach der Situation, genauer nach der Unsicherheit der Unternehmensaufgabe. Während einige grundlegende Koordinationsmechanismen (Hierarchie, Regeln, Zielvorgaben, direkte Weisungen) in nahezu jeder Situation eingesetzt wurden, finden sich die "teureren" Koordinationsmechanismen, wie die Bildung von Teams, Integrationsstellen oder -abteilungen, nur in einer Umgebung hoher Unsicherheit. Insofern bildet die Ordnung nach der Unsicherheit auch eine Ordnung nach den Kosten der Koordinationsmechanismen. ${ }^{6}$ Angesichts ihrer Relevanz erscheint die situative Unsicherheit ein theoretisch und empirisch gut fundiertes Kriterium zur Gliederung der Arten von Koordinationsmechanismen zu sein. Im folgenden werden die Arten von Koordinationsmechanismen knapp zusammenfassend skizziert, unterschieden in administrative, strukturelle und personale.

Innovationsentscheidungen, Wiesbaden, 1987, S. 130 - 131.

1 Schanz, G., Organisationsgestaltung, a.a.O., S. 159. In Bezug auf die Verbindungseinteilungen (vgl. Abb. 2.18) in Anlehnung an Galbraith, J.R., Complex Organizations, a.a.O., S. 48.

2 Frese, E., Organisation, a.a.O., S. 201.

3 March, J.G., Simon, H.A., Organizations, a.a.O., S. 160 - 161.

4 Khandwalla, P., Organizations, a.a.O., S. 549.

5 Van de Ven, A., Delbecq, A., Koenig, R.Jr., Coordination Modes, a.a.O., S. 324.

6 Vgl. Lawrence, P., Lorsch, J., Organization and Environment, a.a.O., Kapitel 6; Galbraith, J.R., Kazanjian, R.K., Strategy Implementation, a.a.O., S.72 - 74. Da die Kosten eines Koordinationsmechanismus mit dem institutionalisierten Koordinationsmedium variieren dürften, ist die Einteilung konsistent mit der von Kieser/Kubicek gewählten Gliederung. 


\subsection{Administrative Koordinationsmechanismen}

Als administrativ seien Koordinationsmechanismen bezeichnet, die eine weitgehend in die Zukunft wirkende, inhaltliche Fixierung des zu koordinierenden Sachverhalts vorsehen. ${ }^{1}$ Gemeinhin wird dabei zwischen Plänen und Programmen unterschieden. ${ }^{2}$ Auf Planung beruhende Koordinationsmechanismen sind konkrete inhaltliche Vorgaben an die Unternehmensteile, z.B. Zielvorgaben, Budgetvorgaben, Mengen- oder Zeitvorgaben. Die Standardisierung ist ein wichtiges, auf Planung beruhendes, administratives Koordinationsinstrument zur Schaffung integrativer Verbindungen. Mintzberg unterscheidet die Standardisierung von Arbeitsprozessen, Arbeitsergebnissen (Outputs) und Fähigkeiten (Skills). ${ }^{3}$ Durch Standardisierung können Schnittstellen besser überwunden und Gleichartigkeiten besser ausgenutzt werden, weil beispielsweise der Materialfluß in der Produktion oder der Personalaustausch zwischen den Unternehmensteilen erleichtert werden. Gegenüber der Planung sind Programme (Standard Operating Procedures) nicht einzelne Vorgaben, sondern auf Dauer angelegte Verfahrensrichtlinien, die auch konditionale Verzweigungen enthalten können, also Verfahrensregeln in Abhängigkeit von vorhersehbaren Umweltsituationen. Dabei besteht ein Programm aus zwei Komponenten, einerseits aus einer Spezifikation der Zukunftszustände, andererseits aus Verfahrensregeln bei Eintreten der Zukunftszustände. ${ }^{4}$ Beispiele für Programme sind Zeitregeln oder zeitliche Vorgaben, Angaben, wann welche Handlungen auszuführen sind, Ergebnisregeln oder Kooperations- und Kommunikationsregeln.

1 Vgl. z.B. March, J.G., Simon, H.A., Onganizations, a.a.O., S. 160.

2 Vgl. z.B. Kieser, A., Kubicek, H., Organisation, a.a.O., S. 113.

3 Mintzberg, H., Structuring of Organizations, a.a.O., S. 3 - 9.

4 Vgl. Kieser, A., Kubicek, H., Organisation, a.a.O., S. 119 - 123; Welge, M.K., Unternehmensführung, Band 2, a.a.O., S. 426. 
Den administrativen Koordinationsmechanismen ist gemein, daß personen- und teilweise situationsunabhängig eine bestimmte Durchführung von Handlungen kodifiziert wird. ${ }^{1}$ Sie bieten sich damit zur Gestaltung einer vertikalen Verknüpfung oder horizontalen Verflechtung im Integrationsbereich nur dann an, wenn im vorhinein hinreichend determinierbar ist, wie die zukünftige der Verbindung auszugestalten ist, d.h. wenn die potentiellen Zukunftskontingenzen spezifiziert und konditionale Handlungsverzweigungen zugeordnet werden können.

\subsection{Strukturelle Koordinationsmechanismen}

Strukturelle Koordinationsmechanismen sind in der Aufbaustruktur der Unternehmung vorgesehene und implementierte Regelungen zur Bildung integrativer Beziehungen. ${ }^{2}$ Bei den strukturellen Koordinationsmechanismen können unterschieden werden die hierarchische Strukturierung der Aufbauorganisation und die Querverbindungen. ${ }^{3}$

Der hierarchische Aufbau der Unternehmung ist ein zentraler Mechanismus zur Schaffung integrativer Verbindungen. Die Koordinationsmedien bei hierarchischen Über- bzw. Unterordnungsbeziehung sind einerseits die direkte persönliche Weisung ${ }^{4}$ andererseits die vielfältigen Formen der (in)formalen Kommunikation und die gegenseitige Abstimmung, die sich in

1 Vgl. Van de Ven, A., Delbecq, A., Koenig, R.Jr., Coordination Modes, a.a.O., S. 323. Die Situationsunabhängigkeit liegt nur insoweit vor, wie die Handlungsausführung nicht konditional in Bezug auf vorhergesehene Zukunftssituationen definiert wurde.

2 Vgl. Welge, M.K., Unternehmensführung, Band 2, a.a.O., S. 432.

3 Vgl. Van de Ven, A., Delbecq, A., Koenig, R.Jr., Coordination Modes, a.a.O., S. 323. Es wird hier bewußt auf die Verwendung der Begriffe "vertikal" für Über- bzw. Unterordnungsbeziehung und "horizontal" für Selbstabstimmung verzichtet, um Verwechslungen mit den Begriffen der horizontalen Verflechtung und der vertikalen Verknüpfung zu vermeiden. Zur Wahl der Begriffe vertikal und horizontal in dieser Arbeit, vgl. Abschnitt B.2.1.2.

4 Vgl. Kieser, A., Kubicek, H., Organisation, a.a.O., S. 113; Welge, M.K., Unternehmensführung, Band 2, a.a.O., S. 416; Dessler, G., Organization Theory Integrating Structure and Behavior, 2 nd. ed., Englewood Cliffs, New Jersey, 1986, S. 151. 
der gemeinsamen Arbeit ergeben. ${ }^{1}$ Die Bildung von Unternehmensteilen als aufbauorganisatorische Einheiten wird sich dabei zweckmäßigerweise an den Interdependenzen, also den Schnittstellen und Gleichartigkeiten, ausrichten. Da die Unternehmung nur nach einem Kriterium pro Unternehmensebene untergliedert werden kann, (bei Matrixorganisation zwei) steht die "Coordination through Departmentation"2 angesichts der Fülle verschiedener Interdependenzen bei der Bildung integrativer Verbindungen nicht im Vordergrund. Vielmehr wird die Bildung vertikaler Verknüpfungen und horizontaler Verflechtungen von Unternehmensteilen immer zunächst an den aufbauorganisatorischen Gegebenheiten (den Unternehmensteilen) ansetzen müssen. Gleichwohl mag sich die zweckmäBige Aufbauorganisation ändern, wenn eine veränderte Bedeutungen von Gleichartigkeiten und Schnittstellen eine Anpassung zweckmäßig erscheinen läßt. Dieser Aspekt ist aufgrund des Integrationspotentials durch Abteilungsbildung bei der Frage strategischer Integration zu berücksichtigen. ${ }^{3}$ Beispielsweise könnte eine kundenbezogene horizontale Verflechtung der Produktgruppen einer Bank erhebliche Positions- und Dauerhaftigkeitsvorteile bringen. Die Bildung solcher horizontalen Verflechtungen ist jedoch kaum möglich, wenn die operativen Unternehmensteile, die die verschiedenen Geschäftsprozesse tragen, nach den einzelnen Produkten zugeschnitten sind. Insofern ist auch die Dekomposition der Unternehmung in ihre Teile ein Integrationsproblem. Die Aufbauorganisation ist dabei eines der Integrationsinstrumente bzw. einer der alternativ möglichen Koordinationsmechanismen. Die Abteilungsbildung als Dekomposition der wie auch immer definierten Gesamtaufgabe der Unternehmung 4 und die Integration können mithin als zwei Seiten einer Medaille interpretiert werden.

1 Vgl. z.B. March, J.G., Simon, H.A., Organizations, a.a.O., 1958, S. 161; Mintzberg, H., Structuring of Organizations, a.a.O., S.49 ff.

2 Dessler, G., Organization Theory, a.a.O., S. 151.

3 Vgl. Kieser, A., Kubicek, H., Organisation, a.a.O., S. 104 - 109.

4 Klassisch: Nordsieck, F., Grundlagen der Organisationslehre, Stuttgart, 1934. 
Querverbindungen sind ein zweiter struktureller Koordinationsmechanismus. Sie sind definiert als strukturelle organisationale Verbindung zwischen Personen in zwei oder mehreren Unternehmensteilen. Die einfachste Form einer Querverbindung ist der direkte Kontakt zwischen dem Management der betroffenen Unternehmensteile. ${ }^{1}$ Mit wachsendem Volumen von abzustimmenden Aktivitäten werden die Unternehmensteile Verbindungsstellen, also Mitarbeiter, mit der laufenden Abstimmung betrauen. Eine Alternative dazu ist das Vorsehen eines Integrators auf einer aufbauorganisatorisch übergeordneten Ebene; ein Extremfall dafür ist die Matrixorganisation.

Die strukturellen Koordinationsmechanismen eröffnen im Vergleich $z u$ den administrativen die Möglichkeit, Anpassungen noch während des zu koordinierenden Prozesses vorzunehmen. Sie setzen also weniger inhaltliche Prädisposition künftiger Ereignisse voraus. Dennoch erfordern sie eine konkrete Institutionalisierung, d.h. eine Definition der $\mathrm{zu}$ beteiligenden Personen sowie des Regelungsinhalts, der über die Querverbindung integriert werden soll.

\subsection{Personale Koordinationsmechanismen}

Personale Koordinationsmechanismen richten sich auf die organisational zu integrierenden Personen. ${ }^{2}$ Dabei sei unterschieden zwischen der Teambildung und Selbstabstimmung einerseits und der kultur- bzw. wertorientierten Koordination andererseits.

1 Vgl. dazu und zum folgenden Galbraith, J.R., Complex Organizations, a.a.O., S. 48 ff. und $93 \mathrm{ff}$.

2 Vgl. Welge, M.K., Unternehmensführung, Band 2, a.a.O., 1986, S. 415; Khandwalla, P., Organizations, a.a.O., 1977, S. 549 ff; Van de Ven, A., Delbecq, A., Koenig, R.Jr., Coordination Modes, a.a.O., S. 323. 
Die Bildung von Teams ist ein personaler Koordinationsmechanismus mit struktureller Orientierung. ${ }^{1}$ Dabei ist von der formalen, geplanten $\mathrm{Zu}$ sammenarbeit die informale, ungeplante Zusammenarbeit $\mathrm{zu}$ unterscheiden, die für improvisierte und Ad-hoc-Abstimmungen arbeitsbezogener Probleme eingesetzt wird. ${ }^{2}$ Im Zusammenhang mit der Bildung von Teams steht die Koordination durch Selbstabstimmung, verstanden als Abstimmung durch wechselseitige Manipulation und Adaption der Entscheidungsträger. $^{3}$

Das vorgelegte Kontinuum von Koordinationsmechanismen (administrative, strukturelle, personale) richtet sich nach der Notwendigkeit der Prädisposition zukünftiger Zustände. Mit ungeplanten Ad-hocTeams erscheint das Ende des Kontinuums erreicht. Eine inhaltliche Prädisposition $\mathrm{zu}$ integrierender Beziehungen mag dabei allenfalls noch dadurch gegeben sein, daß Grenzen für die Zuständigkeiten bei Selbstkoordination gesetzt werden, etwa durch Festlegung der Kommunikationskanäle oder der Entscheidungskompetenzen. ${ }^{4}$ Mit der kultur- oder wertorientierten Koordination wird demgegenüber auf Prädisposition zukünftiger Ereignisse verzichtet. Vielmehr wird Integration über ein allgemeines, gemeinsames Grundverständnis angestrebt, also gewissermaßen dadurch, daß sich die in verschiedenen Unternehmensteilen tätigen Personen zwar freiwillig, aber dennoch "indoktrinativ" geleitet an die Zielfunktion der Unternehmung anpassen. 5

1 Teams werden unter personalen Koordinationsmechanismen subsumiert. Von ihrem Einsatz wird erwartet, daB durch die im Team entstehenden Diskussionen und interpersonalen Prozesse Lösungen für das Integrationsproblem entwickelt werden. Alternativ wäre es möglich, Teams als strukturelle Koordinationsmechanismen mit personaler Orientierung aufzufassen. Auch bei diesem Verständnis liegen sie innerhalb des gesamten Kontinuums von Koordinationsmechanismen den personalen am nächsten.

2 Vgl. Hage, J., Communication and Organizational Control, New York, New York, 1971, S. 151.

3 Vgl. Welge, M.K., Unternehmensführung, Band 2, a.a.O., S. 423, Kieser, A., Kubicek, H., Organisation, a.a.O., S. 115.

4 Vgl. Kieser, A., Kubicek, H., Organisation, a.a.O., S. 116.

5 Vgl. Mintzberg, H., Structuring of Organizations, a.a.O., S. 97 ff.; Welge, M.K., Unternehmensführung, Band 2, a.a.O., S. 419 ff. 


\subsubsection{Kontrakte}

Die Kontrakte sind das Integrationsinstrument zur kontraktuellen Bildung horizontaler Verflechtungen und vertikaler Verknüpfungen. Aus Sicht der ökonomischen Organisationstheorie ${ }^{1}$ determinieren Kontrakte weitgehend das Geschehen der Unternehmung. So wird die Unternehmung definiert als kontraktuelle Fiktion, als Geflecht (Nexus) von Kontrakten, die sowohl zwischen den Faktoreigentümern (Mitarbeiter, Lieferanten, Kapitalgeber) als auch mit den Abnehmern geschlossen werden. ${ }^{2}$ Ein Kontrakt kann definiert werden als die Beziehung zwischen Parteien, die auf den Prozeß zukünftigen Tausches gerichtet ist. ${ }^{3}$ Kontrakte spezifizieren Rechte und Pflichten der Kontraktparteien, insbesondere die von Zukunftskontingenzen abhängigen gegenseiten Zahlungen und Leistungen. Die geschlossenen Kontrakte werden dabei völlig verschiedener Art sein, je nachdem, worauf sie sich richten und welcher Art die Geschäftsaktivitäten im betrachteten Integrationsbereich sind. Mit Arbeitnehmern wird die Unternehmung Arbeitsverträge schließen, mit Lieferanten Kauf- und Rahmenlieferverträge, mit Kapitalgebern Kredit- und Gesellschaftsverträge, mit Abnehmern z.B. Kaufverträge.

1 Gegenstand der ökonomischen Organisationstheorie (Organizational Economics) ist die Anwendung (neoklassischer) ökonomischer Methoden auf die Organisation der Unternehmung. Bausteine der ökonomischen Organisationstheorie sind der PrinzipalAgenten-Ansatz, Transaktionskostenansatz, evolutorische Ansätze und die Ökonomie der Unternehmensstrategie. Einen Überblick über die Ansätze geben Barney, J.B., Ouchi, W.G., Organizational Economics. San Francisco, London, 1986; Picot, A., Ökonomische Theorien der Onganisation - Ein Überblick über neuere Ansätze und deren betriebswirtschaftliches Anwendungspotential, in: Betriebswirtschaftslehre und ökonomische Theorie, Jahrestagung des Verbandes der Hochschullehrer für Betriebswirtschaft vom 6. Juni 1990, Ordelheide, D., Rudolph, B., Büsselmann, E., (Hrsg.), Stuttgart, 1991, S. 143 - 170.

Vgl. Jensen, M.C., Meckling, W.H., Theory of the Firm: Managerial Behavior, Agency Costs, and Ownership Structure, in: Journal of Financial Economics, Vol. 3, No. 4, 1976, S. 305 - 360, wiederabgedruckt in: Barney, J.B., Ouchi, W.G., Organizational Economics, a.a.O., S. 219 ff. Diese Definition der Unternehmung wird jedoch nicht von allen Ansätzen der ökonomischen Organisationstheorie geteilt. Vgl. 2.B. Williamson, O.E., Economic Organization, a.a.O., S. 29 ff. Der Streit über die korrekte Definition der Firma ist für die folgenden Überlegungen jedoch von nachrangiger Bedeutung und wird daher hier nicht diskutiert.

3 Vgl. MacNeil, New Social Contract, a.a.O., S.4. 
Die zu betrachtenden Kontrakte umfassen die konstitutiven und laufenden Kontrakte, in denen die Rechte und Pflichten der am Geschäftsprozeß Beteiligten spezifiziert sind, dabei (aus ökonomischer Perspektive) insbesondere die von Zukunftskontingenzen abhängigen Zahlungen. In der Literatur besteht weitgehend Einigkeit in der Unterscheidung dreier Klassen kontraktueller Arrangements: Erstens der Marktkontrakt, d.h. kurzfristige, auf den Marktaustausch bezogene Kontrakte, z.B. Kaufverträge, die inhaltlich vollständig einen Tauschgegenstand und die Tauschbedingungen spezifizieren; zweitens die Unternehmung, d.h. konstitutive, langfristige, offene Kontrakte, die oft erst nach Abschluß inhaltlich näher auszufüllen sind, wie Gesellschafts- und Arbeitsverträge, sowie drittens die relationalen Kontrakte, d.h. Zwischenformen der beiden vorgenannten. ${ }^{1}$

Grossman und Hart betrachten als (kontraktuell) integriert diejenigen Faktoren, die die Eigentümer der Unternehmung direkt oder indirekt besitzen bzw. entscheidend kontrollieren, während als (kontraktuell) nicht integriert diejenigen Faktoren gelten, die sich nicht unter der Kontrolle der Unternehmung befinden. ${ }^{2}$ Aus ökonomischer Perspektive ist dabei die Eingliederung von Produktionsfaktoren unter die gleiche Zielfunktion das entscheidende Merkmal der kontraktuellen Integration. Das Ausmaß von kontraktueller (vertikaler oder horizontaler) Integration im Integrationsbereich kann also danach operationalisiert werden, ob die betrachteten Geschäftsaktivitäten durch den Einsatz eigener Produktionsfaktoren oder durch Produktionsfaktoren in fremdem Eigentum (bzw. Besitz, Kontrolle) erbracht werden - mit einem Kontinuum aus Zwischenformen.

1 Vgl. MacNeil, New Social Contract, a.a.O. S. 4; Williamson, O.E., Economic Institutions, a.a.O., S. 15; Williamson, O.E., Economic Organization, a.a.O., S. 20 - 32.

2 Grossman, S.J., Hart, O.D., The Costs and Benefits of Ownership: A Theory of Vertical and Lateral Integration, in: Journal of Political Economy, Vol. 94, 1986, S. 693 ff. Grossman und Hart definieren die Unternehmung anhand der Aktiva, die die Unternehmung besitzt, wobei sie mehr auf die Kontrolle der Aktiva als auf das tatsächliche Eigentumsrecht abstellen. Dies erlaubt die relativ elegante Definition eines Faktors bzw. Aktivums als integriert versus nicht integriert. 
Zur Unterscheidung von Markt, Unternehmung und relationalen Kontrakten verwendet MacNeil acht Merkmale: (1) Spezifität und Meßbarkeit, (2) Quellen kontraktueller Solidarität, (3) Planung, (4) Aufteilung von Nutzen und Lasten, (5) Obligationen, (6) Fungibilität, (7) Einstellungen, (8) Macht, Hierarchie und Anweisung. ${ }^{1}$ Diese werden im folgenden herangezogen, um Markt und Unternehmung als Extrempunkte eines Kontinuums kontraktueller Arrgangements zu charakterisieren.

\subsection{Marktkontrakte}

Als Marktkontrakt soll ein Kontrakt verstanden werden, in dem keine über den einfachen Tausch von Gütern hinausgehende Beziehung zwischen den kontraktschließenden Parteien besteht. ${ }^{2}$ In Bezug auf die genannten Kriterien ist der Marktkontrakt wie folgt zu beschreiben (vgl. Abb. B.2.19).

Der Inhalt eines Marktkontrakts umfaßt die genaue, vollständige Beschreibung des Tauschgegenstandes bzw. der Rechte und Pflichten der tauschenden Parteien. Voraussetzung dazu ist deren Meßbarkeit. Eine Verletzung des Marktkontrakts bedeutet eine Verletzung extern definierter Regeln, nämlich des Vertragsrechts, das somit die Quelle der Solidarität der Kontraktparteien ist. Den Inhalt des Marktkontrakts legt häufig unilateral der Anbieter einer Leistung fest, und zwar vollständig, bindend fixiert und nur auf den einen Zeitpunkt des Tauschs selbst gerichtet. Dabei sind die Rechte und Pflichten oder der Nutzen und die Lasten den Kontraktparteien eindeutig zugeordnet. Der Inhalt von Verpflichtungen aus dem Kontrakt beruht auf konkret gegebenen, spezifisch formulierten Versprechen. Die Sanktionen bei Verletzung der Verpflichtungen sind sehr präzise (extern) formuliert. Marktkontrakte haben einen kurzen Zeithorizont, Anpassungen oder Änderungen des Kontraktinhalts werden nicht erwartet. Der

1 MacNeil, New Social Contract, a.a.O., S. 21.

2 Goldberg, V.P., Toward an Expanded Economic Theory of Contract, in: Journal of Economic (Fußnote wird auf der nächsten Seite fortgesetzt) 
Tauschgegenstand Marktkontrakte ist prinzipiell fungibel und insoweit unabhängig von den tauschenden Personen. Die Kontraktparteien werden sich auf nur geringe Gemeinsamkeiten einstellen, vielmehr Zielkonflikte ("Nullsummenspiel") erwarten. Neben dem Tausch liegt keine Macht oder Anweisungsbefugnis einer Seite vor.

\begin{tabular}{|c|c|}
\hline (1) Vollotiindigkek und Mesbarkatt & Vollige, gonaus Bestimmeneit \\
\hline (2) Quellon kontraltueller Solidarkint & Extern: Vertragerecht \\
\hline (3) Planung & Unilateral, volletändig, bindend, einperiodisch \\
\hline (4) Auftellung von Nutzen und Leoten & Prizise bestimmt \\
\hline (5) Quelle von Verpelilehtungen & Explizito Vorsprechen, oxternes Recht \\
\hline (6) Funglbilikit / Trandefmogllehkelt & gegeben \\
\hline (n) Erwartungen & Zielkonflike, kurzer Zoithoriz., koine Anpass. \\
\hline (8) Hbrarchlo und Anwoloung & Keino \\
\hline
\end{tabular}

Abb. B.2.19: Charakterisiorung von marktbezogenen Kontrakten

Marktkontrakte sind inhaltlich vollständig und kurzfristig. ${ }^{1}$ Der Preis ist die einzige Variable. Im Preis ist sämtliche verfügbare Information der Marktteilnehmer enthalten bzw. gespiegelt. ${ }^{2}$ Veränderte Umweltbedingungen schlagen sich (sofort) in Preisänderungen nieder. Dies verursacht unmittelbare Anpassungen. Daraus resultiert erheblicher Anreiz, Produktionsfaktoren bestmöglich einzusetzen. ${ }^{3}$ Jedoch nähern sich die Interessen (Zielfunktionen) der Vertragsparteien durch einen Marktkontrakt nicht an. Bei Vorliegen gegensätzlicher Zielfunktionen kann aber die Bildung vertikaler Verknüpfungen und horizontaler Verflechtungen problematisch sein, falls Zielkonflikte zwischen dem gemeinsamen Integrationsziel und den Zielfunktionen einer oder beider Parteien vorliegen. Die erforderliche

Issues, Vol. 10, 1976, S. 49.

1 Vgl. Holmstrom, B.R., Tirole, J., Theory of the Firm, in: Handbook of Industrial Organization, (Hrsg.), Schmalensee, R., Willig, R., Amsterdam, New York, 1989, S. 68 ff.; Hart, O.D., Incomplete Contracts and the Theory of the Firm, in: Journal of Law, Economics, and Organization, Vol.4, 1988, S. 120 ff.

2 Vgl. Hayek, Fr.A. von, The Use of Knowledge in Society, in: American Economic Review, Vol. 35, 1945, S. 525 ff.

3 Vgl. Williamson, O.E., Economic Organization, a.a.O., S. 20 - 22. 
Berücksichtigung von Interdependenzen findet dann nur so lange statt, wie Pareto-Verbesserungen erzielbar sind.

\subsection{Unternehmenskonstituierende Kontrakte}

Die die Unternehmung konstituierenden Kontrakte sind das Gegenteil der reinen Marktkontrakte (vgl. Abb. B.2.20).

\begin{tabular}{|c|c|}
\hline (1) Vollethndigkelt und Mobbarkolt & Unvallstholig, ErsetzmaBstebo \\
\hline (2) Quellen kontraktuelbr solideritit & Intem zukônfüge Zusammonarbelt, externo Restrikt. \\
\hline (3) Plamung & Multilated, unvollatelndg, ungenau, lengfilstig \\
\hline \multicolumn{2}{|l|}{ (4) Auftellung von Mutzen und Laston } \\
\hline (5) Quelle von Verpillchungen & Implzite Faktoren, explziteVersprechon, extermes Pucht \\
\hline (6) Funglbilnht / Trenefermb̈gllchk dit & Sltuatlv verschieden \\
\hline (7) Erwartungen & Ger. Zelkonfl., langer Zuithoriz., Anpass.mbgl. \\
\hline (8) Herarchie und Anweioung & Bedeutsan \\
\hline
\end{tabular}

Abb. B.2.20: Charakterisierung der die Unternehmung konstituierenden Kontrakte

Im Gegensatz zum marktbezogenen Tausch ist der die Unternehmung konstituierende Kontrakt auf eine langfristige Beziehung ausgelegt, beispielsweise ein Gesellschaftsvertrag oder ein Arbeitsvertrag. ${ }^{1}$ Unternehmenskontrakte sind unvollständig und werden erst nach Abschluß im Laufe ihres Bestehens inhaltlich ausgefüllt, selbst dies nur teilweise durch explizit vereinbarte Regelungen. ${ }^{2}$ Gegenüber dem marktbezogenen Kontrakt ist es in der Unternehmung häufig unmöglich, den eigentlichen Tauschgegenstand (Faktorgrenzprodukt) präzise zu messen und vollständig zu beschreiben. ${ }^{3}$ Die kontraktuelle Solidarität in der Unternehmung wird durch interne Mechanismen und die auf Dauer angelegte Reziprozität

1 Ein Arbeitsvertrag wird nicht im rechtlichen Sinne als konstitutiv angesehen werden können, ist jedoch hinsichtlich mehrerer Dimensionen dem Gesellschaftsvertrag sehr ähnlich.

2 Vgl. Hart, O.D., Incomplete Contracts, a.a.O., S. 121 ff.

3 Vgl. z.B. Alchian, A.A., Demsetz, H., Production, Information Costs and Economic (FuBnote wird auf der nächsten Seite fortgesetzt) 
gewährleistet, externe Restriktionen wirken nur in Ausnahmefällen (Arbeitsrecht, Gesellschaftsrecht). Die Planung von Geschäftsaktivitäten der Unternehmung erfolgt multilateral und ist langfristig ausgerichtet; sie kann daher nur ungenau und unvollständig sein. Ein Gesellschaftskontrakt regelt deshalb kaum, wie die Planung selbst inhaltlich ausgefüllt werden soll, sondern gibt eher Grundregeln und Prozesse vor, nach denen künftige Pläne spezifiziert werden. Dabei wird es nicht immer gelingen, die Aufteilung künftiger Nutzen und Lasten genau zu fixieren. Das Eingehen von Verpflichtungen innerhalb der Unternehmung erwächst nicht nur aus expliziten Versprechen, sondern auch aus impliziten, aus der Geschichte der Organisation und der in ihrem Umfeld sich ergebenden Faktoren. Auch in der Unternehmung können Faktoren fungibel sein, also etwa Mitarbeiter, die Aufgaben von anderen übernehmen. Fraglich ist jedoch, ob eine externe Nachfrage nach den Faktoren der Unternehmung außerhalb der Unternehmung besteht.

Die Erwartungen an unternehmenskonstituierende Kontrakte mit langem Zeithorizont unterliegen einem gewissen Verständnis von Gemeinsamkeit. Deswegen werden sich durch Schließen eines Arbeits- oder Gesellschaftskontraktes die Interessen (die Zielfunktionen) der Kontraktparteien tendenziell annähern. Dabei erwarten beide Parteien, daß künftige Anpassungen gegenseitig erforderlich sein werden, wenn sich die Umweltsituation unvorhersehbar ändert. Nicht im Kontrakt spezifizierte Einzelheiten können überwiegend durch einseitige, hierarchische Anweisung ausgefüllt werden, z.B. durch eine Dienstanweisung im Rahmen eines Arbeitsvertrags. 1

Die Unternehmung als institutionelles Arrangement ist durch die sie konstituierenden Kontrakte charakterisiert. Ihre Langfristigkeit, Anpassungsfähigkeit und ihr Abstimmungspotential begründen kontraktuelle horizon-

Organisation, in: American Economic Review, Vol. 62, 1972, S. 779 ff.

1 Im Beispiel ergänzen sich kontraktuelle und organisatorische Integrationsmechanismen. 
tale Verflechtungen und vertikale Verknüpfungen. Mit der kontraktuellen Integration in die Unternehmung werden jedoch die Anreiz- und Allokationsvorteile des Marktes aufgegeben. ${ }^{1}$ Anstelle präziser Preisinformationen rücken mehr oder weniger befriedigende Ersatzmaßstäbe. Grenzprodukte einzelner Faktoren sind kaum ermittelbar. ${ }^{2}$ Williamson bezeichnet die Unternehmung daher als "letzte Rettung", die nur eingesetzt werde, wenn alles andere fehlschlägt. ${ }^{3}$

\subsection{Relationale Kontrakte}

Zwischen den skizzierten Extrempunkten von Markt und Unternehmung liegt ein Kontinuum kontraktueller Arrangements. MacNeil bezeichnet diese als relationale Kontrakte. ${ }^{4}$ Relationale Kontrakte liegen in Bezug auf jedes MacNeilsche Kriterium zwischen Markt und Unternehmung. Insofern wird von einer eigenen Charakterisierung relationaler Kontrakte abgesehen. Aus der vorstehenden Diskussion kann zusammenfassend festgehalten werden, daß relationale Kontrakte die Vorteile von Marktkontrakten und unternehmenskonstituierenden Kontrakten, nämlich die Anreizwirkungen einerseits und die administrativen Möglichkeiten zur Integration andererseits, prinzipiell kombinieren können.

Kontraktuelle Integration zielt auf Annäherung der Zielfunktionen der beteiligten Kontraktpartner. Dabei können zwei grundsätzlich verschiedene Strategien unterschieden werden, diese Annäherung zu erreichen. Die klassische Strategie ist das direkte Zusammenlegen des Eigentums an aufeinander angewiesenen Produktionsfaktoren, also z.B. die Übernahme ei-

1 Vgl. Grossman, S.J., Hart, O.D., Ownership, a.a.O., S. 716; Hart, O.D., Incomplete Contracts, a.a.O., S. 134 ff; Holmstrom, B.R., Tirole, J., Theory of the Firm, a.a.O., S. 74 75.

2 Vgl. Alchian, A.A., Demsetz, H., Economic Organisation, a.a.O., S. 779 ff; Williamson, O.E., Economic Organization, a.a.O., S. 18-21, Grossman, S.J., Hart, O.D., Ownership, a.a.O., S. 710 ff.

3 Vgl. Williamson, O.E., Economic Organization, a.a.O., S. 20. 
nes Lieferanten durch einen Hersteller. ${ }^{1}$ Dadurch wird der Lieferant der Zielfunktion des Herstellers unterstellt. Der Lieferant mag ein Commitment in eine Spezialtransportmaschine eingegangen sein. Die kontraktuelle Integration sichert die Rente aus diesem Commitment ab. Eine Abschöpfung ist für den Hersteller dann weder möglich noch ökonomisch sinnvoll.

Diese klassische Strategie zur Annäherung der Zielfunktion hat jedoch den entscheidenden Nachteil, daß durch vollständige kontraktuelle Integration die Marktanreize und die Informationen über den Zustand der übernommenen Einheit verlorengehen. ${ }^{2}$ Eine neuere in der Literatur zunehmend diskutierte Strategie der Annäherung der Zielfunktionen zweier oder mehrerer Unternehmen ist das Netzwerk, eine langfristige Beziehung, die auf impliziten 'Kontrakten' beruht, ohne auf explizite kontraktuelle Integration zurückzugreifen. ${ }^{3}$ Ein Netzwerk ist damit im Vergleich zum Marktkontrakt eine stark veränderte Tauschbeziehung der beteiligten Parteien. Diese Veränderung kann nach den acht Merkmalen von MacNeil beschrieben werden. Das Netzwerk könnte so definiert werden als eine Veränderung aller acht Merkmale, ohne ein gemeinsames Eigentum von Produktionsfaktoren vorzusehen.

Relationale Verträge bilden ein Kontinuum zwischen Markt und Unternehmung im Hinblick auf diese Merkmale. Einige Formen von relationa-

4 MacNeil, New Social Contract, a.a.O., S. 25 - 35.

1 Grossman, S.J., Hart, O.D., Ownership, a.a.O., S. 693.

2 Vgl. Williamson, O.E., Economic Organization, a.a.O., S. 20, Grossman, S.J., Hart, O.D., Ownership, a.a.O., S. 716; Hart, O.D., Incomplete Contracts, a.a.O., S. 134 ff; Holmstrom, B.R., Tirole, J., Theory of the Firm, a.a.O., S. 74 ff.

3 Jarillo, J., Ricart, P., Networks, a.a.O., S. 83, Vgl. Miles, R.E., Snow, Ch.C., Fit, Failure and the Hall of Fame, in: California Management Review, Vol. 26, No. 3, 1984, S. 10 - 28; Thorelli, H.B., Networks, a.a.O., S. 37 - 51; Farmer, D.H., MacMillan, K., Voluntary Collaboration vs. "Disloyalty" to suppliers, in: Journal of Purchasing in Materials Management, Vol. 12, No. 4, 1976, S. 3 - 8; MacMillan, K., Farmer, D.H., Boundaries of the Firm, a.a.O., S. 277 - 285. Einen Überblick gibt Delfmann, W., Das Netzwerkprinzip als Grundlage integrierter Unternehmensführung, in: Der Integrationsgedanke in der Betriebswirtschaftslehre: Helmut Koch zum 70. Geburtstag, (Hrsg.), Delfmann, W., Kuhn, A., Wiesbaden, 1989, S. 87 - 113. 
len Kontrakten sind, ohne Anspruch auf Vollständigkeit, in die Systematik der Abb. B.2.21 zwischen Markt und Unternehmung eingeordnet.1

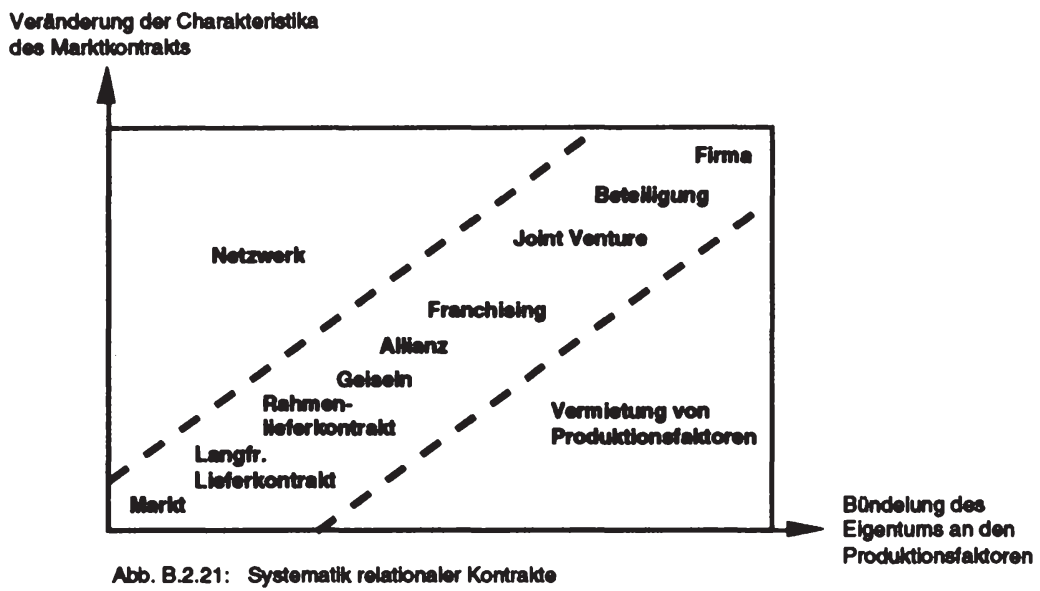

Dabei wird an der Ordinate danach unterschieden, wie weit sich der relationale Kontrakt vom Marktkontrakt (tendenziell) entfernt und den Charakter eines unternehmenskonstituierenden Kontrakts annimmt, gemessen an den MacNeilschen Kriterien. An der Abszisse wird abgetragen, wie stark das Eigentum an kritischen Produktionsfaktoren gebündelt ist. Dabei ergibt sich innerhalb der gestrichelten Linien ein Kontinuum "üblicher" kontraktueller Arrgangements. Diese seien knapp skizziert.

Langfristige Lieferverträge ${ }^{2}$ können wie Marktkontrakte auf genauen $\mathrm{Zu}$ kunftsspezifikationen beruhen und insoweit auf externen Mechanismen kontraktueller Solidarität aufbauen. Dazu kann aber auch schon die

1 Die Einondnung hängt dabei nicht nur von der Kontraktform, sondern auch von der konkreten Situation ab. Insofern ist diese Reihung nur eine mögliche. Sie soll Tendenzen aufzeigen, nicht Kontrakte präzise charakterisieren.

2 Vgl. z.B. Klein, B., Crawford, R., Alchian, A.A., Vertical Integration, a.a.O., S. 302. 
(interne) Drohung, den Kontrakt zu kündigen, hinreichen. M.a.W. der langfristige Kontrakt bietet mindestens ebensoviel Anreiz, die kontraktuelle Solidarität $\mathrm{zu}$ wahren, wie die vertraglich fixierte Verpflichtung. ${ }^{1} \mathrm{Da}$ mit werden die Charakteristika von Markt- und Unternehmenskontrakt verbunden.

Eine besonders dauerhafte Form langfristiger Lieferverträge sind die Rahmenlieferkontrakte (Subcontracting), wie sie etwa in der Automobilindustrie häufig vorkommen. Dabei wird eine kontraktuelle Integration vermieden, die die Anreizwirkung von Märkten reduziert, aber dennoch für eine feste Dauer ein Liefervolumen, ein Mindestliefervolumen oder andere Parameter vereinbart. Damit kann der Lieferant etwaig erforderliche Commitments eingehen, ohne die Abschöpfung durch den Bezieher fürchten zu müssen.

Das Franchising sieht eine Zusammenlegung von Produktionsfaktoren verschiedener Eigentümer vor. Gegenüber den Rahmenlieferverträgen ist damit der Zeithorizont der Beziehung länger, wobei die Planung im wesentlichen noch unilateral ist (z.B. standardisierte Schnellrestaurants) und die Zukunftskontingenzen weitgehend inhaltlich ausgefüllt sind.

Ein stärkeres bilaterales Planen, eine Verstärkung der Quellen interner Solidarität und eine Verlängerung des Zeithorizonts tritt auf, wenn die Kontraktpartner gegenseitig "Geiseln" stellen.2 Die Gestellung von Geiseln liegt vor, wenn beiderseits glaubwürdige Commitments (credible Commitments) eingegangen werden, mit denen sich die Tauschpartner gegenseitig binden. Commitments sind definiert durch starre Faktoren, entgangene Optionen und organisationale Trägheit. Haben zwei Organisationen ein Commitment in eine von beiden angestrebte integrative Verbindung

1 Williamson sieht in dem Beharren auf Vertragstexten bei offensichtlichem Verstoß gegen den "Geist" des Vertrags, etwa angesichts veränderter Umweltsituation, eine form opportunistischen Verhaltens. Vgl. Williamson, O.E., Economic Organization, a.a.O., S. 34. 
vorgenommen, werden beide stärker daran festhalten als ohne Commitment. Damit erübrigt sich die totale kontraktuelle Integration. Jede Seite kann in den Grenzen der Bindungswirkung des Commitments auf die Fortsetzung der Beziehung setzen, da per Definition nach Eingehen eines Commitments eine Fungibilität nicht mehr gegeben ist. Damit ist die Wettbewerbsposition aus einem eigenen Commitment vor der Abschöpfung durch die Gegenseite dadurch abgesichert, daß auch die Gegenseite ein Commitment eingeht, das abschöpfbar wäre.

Eine stärkere bi- bzw. multilaterale Planung der kontraktuellen Beziehung liegt vor, wenn die Kontraktparteien gemeinsam Eigentum bzw. Verfügungsrechte an Produktionsfaktoren erwerben. Entsprechende Arrangements sind z.B. Allianzen, Joint Ventures, direkte Beteiligungen. Grundsätzlich kann die Beteiligung der Kontraktpartner am Kapital des jeweils anderen unterschieden werden von der gemeinsamen Beteiligung am Eigenkapital eines dritten Unternehmens. In beiden Fällen kommt es zu einer Annäherung der Zielfunktion zwischen den Beteiligten.

Ein auf der physischen Hülse einer Unternehmung aufbauender relationaler Kontrakt ist die Vermietung von Produktionsfaktoren (Inside Contracting). Dabei werden die meisten kritischen Produktionsfaktoren (z.B. die Gebäude, der Maschinenpark, die Büroinfrastruktur) kontraktuell in die Unternehmung integriert, jedoch an "Mitarbeiter" verleast oder vermietet. Dabei handelt es sich um den Versuch, Marktmechanismen weitgehend in die Unternehmung einzuführen, ohne grundsätzliche Potentiale für vertikale Verknüpfungen und horizontale Verflechtungen aufzugeben. Williamson hat auf die schwerwiegenden Probleme dieser Kontraktform hingewiesen, beispielsweise die Übernutzung des Maschinenparks, schwache Anreize für Produktinnovation u.a. ${ }^{1}$ Diese Probleme beruhen darauf, daß Faktoren kontraktuell in die Unternehmung integriert werden, ohne daß

2 Vgl. zum folgenden Williamson, O.E., Economic Institutions, a.a.O., S. $190 \mathrm{ff}$.

1 Vgl. Williamson, O.E., Markets and Hierarchies, a.a.O., S. 96 - 97. 
die in hinreichendem Ausmaß unternehmenskonstituierenden Charakteristika genügen. Die interne Kontrahierung wurde daher "südöstlich" des klassischen Kontinuums relationaler Verträge in die Systematik der Abb. B.2.21 eingeordnet.

Marktbezogene Anreize können sich auch aus der Mitarbeiterbeteiligung an der Unternehmung ergeben. Die dabei geschlossenen Kontrakte sind weitgehend wie die der Unternehmung charakterisierbar. Daraus ergibt sich ein Meßbarkeitsproblem angesichts der Unvollständigkeit des Kontraktes. Gegenüber dem reinen Dienstkontrakt liegt außerdem das Problem der Risikotransformation von der Unternehmung auf den Mitarbeiter vor, das die Wirkung von Anreizsystemen begrenzt.1

Das Netzwerk verfügt über MacNeilsche Charakteristika, die beispielsweise einem Joint Venture ähneln, bei Eigentumsverhältnissen, die beispielsweise einem langfristigen Kaufkontrakt ähneln.2 Mitglieder solcher Netzwerke sind selbständige (Klein-)Unternehmen, die durch enge horizontale Verflechtungen und vertikale Verknüpfungen verbunden sind, ohne kontraktuell bzw. in Form von Eigentum integriert zu sein. ${ }^{3}$ Dabei konzentriert sich jedes Mitgliedsunternehmen auf einige wenige Aktivitäten. Der Verbund entsteht durch in langfristigen Beziehungen aufgebaute Koordinationsmechanismen, die durch beträchtlichen Einsatz verschiedener KIS unterstützt werden. 4 Insofern werden Commitments in netzwerkspezifische Faktoren eingegangen, die der Stellung von Geiseln entsprechen. Eine Absicherungsstrategie mildert jedoch die Risiken der Abschöpfung. Neben

1 Vgl. z.B. Laux, H., Grenzen integrativer Anreizsysteme, in: Der Integrationsgedanke in der Betriebswirtschaftslehre: Helmut Koch zum 70. Geburtstag, (Hrsg.), Delfmann, W., Kuhn, A., Wiesbaden, 1989, S. 202 ff.

2 Vgl. Jarillo, C. J., Ricart, J.E., Sustaining Networks, a.a.O., S. 83;. Miles, R.E., Snow, Ch.,C., Fit, a.a.O., S. 10 - 28; Thorelli, H.B., Networks, a.a.O., S. 37 - 51; Farmer, D.H., MacMillan, K., Voluntary Collaboration, a.a.O., S. 3 - 8; MacMillan, K., Farmer, D.H., Boundaries of the Firm, a.a.O., S. 277 - 285.

3 Konsynski, B. R., McFarlan, F. W., Information Partnerships, a.a.O., S. 114; Johnston, H.R., Lawrence, P.R., Vertical Integration, a.a.O., S. 94 ff.

4 Vgl. Malone, T., Yates, J, Benjamin, R. I. Electronic Markets, a.a.O., S. 166 und S. 169 ff; Johnston, H.R., Lawrence, P.R., Vertical Integration, a.a.O., S. 94 - 101. 
der langfristigen Weiterführung der Beziehung ist dies das Vorsehen potentieller Reservepartner, ${ }^{1}$ mit denen eingegangene Commitments weiter nutzbar sind.

Jeder relationale Kontrakt kann auf die Bildung vertikaler Verknüpfungen oder horizontaler Verflechtungen ausgerichtet werden bzw. diese konstituieren. Ein Grundproblem des marktbezogenen Tauschs ist die mangelnde Absicherbarkeit von Renten aus Commitments bzw. spezifischen Investitionen gegen die Abschöpfung. Kontraktuelle Integration führt ihrem Ausmaß entsprechend zu einer gewissen Angleichung der Zielfunktionen der Kontraktparteien. Renten werden dadurch abgesichert, daß eine langfristige Bindung eingegangen wird, in der eine gemeinsame langfristige Anpassung an zukünftig sich ändernde Zustände möglich wird.

Angesichts des nun erarbeiteten Raumes von Entscheidungsalternativen für die Wahl der drei Integrationsinstrumente erhebt sich die Frage, ob sich bereits allgemeine Aussagen darüber treffen lassen, wie sich dieser Raum eingrenzen läßt. Ansatzpunkte hierzu sind aus dem Zusammenhang der Integrationsinstrumente mit den situativen Bedingungen im Integrationsbereich zu suchen.

\subsubsection{KIS und situative Bedingungen}

Das wichtigste Merkmal zur Unterscheidung von KIS ist die Strukturiertheit der abzuwickelnden Geschäftsaktivität. Diese korrespondiert mit dem Volumen der Geschäftsaktivitäten im Integrationsbereich. Das Volumen von Geschäftsaktivitäten im Integrationsbereich ist durch die Anzahl, die

1 Vgl. Johnston, H.R., Lawrence, P.R., Vertical Integration, a.a.O., S.101. 
Frequenz und die Komplexität definiert. Aus hoher Anzahl und/oder Frequenz ergeben sich Wiederholungen, diese ermöglichen Programmierung bzw. Strukturierung. ${ }^{1}$ Insofern ist die situative Bedingung Volumen von hoher Bedeutung für die KIS. Dabei erscheint es sinnvoll anzunehmen, daß mit höherer Anzahl und Frequenz der Geschäftsaktivitäten im Integrationsbereich, stärker strukturierte Kommunikations- und Informationssysteme eingesetzt werden, um einen hohe Integrationsgrad der vertikalen Verknüpfungen und horizontalen Verflechtungen zu erreichen. Dies gilt insbesondere bei gleichzeitig geringer Unsicherheit. Ein hohes Volumen ist jedoch nicht unbedingt durch eine hohe Anzahl gegeben, vielmehr kann ein hohes Volumen auch durch hohe Komplexität bedingt sein.

\subsubsection{Koordinationsmechanismen und situative Bedingungen}

Von den personalen über strukturelle bis zu den administrativen Koordinationsmechanismen nimmt zwangsläufig die Notwendigkeit zur Prädisposition zukünftiger Zustände zu. Deswegen muß für den Einsatz administrativer Koordinationsmechanismen die Möglichkeit bestehen, im vorhinein Zukunftskontingenzen zu spezifizieren und angemessene Lösungstechniken zuzuordnen. Ein daraus ableitbarer Zusammenhang zwischen Koordinationsmechanismen ist auch empirisch belegt. Van de Ven, Delbeqc und Koenig messen den Einsatz von Koordinationsmechanismen in Abhängigkeit von der Unsicherheit. ${ }^{2}$

Bei den administrativen Koordinationsmechanismen werden die Programme (Rules) und die Planung (Plans) unterschieden, bei den strukturellen die Hierarchie (Vertical Channel) und der laterale Austausch (Horizontal Channel) ${ }^{3}$ und bei den personalen das geplante und das unge-

1 Vgl. Simon, H. A., Management, a.a.O., S. 5.

2 Vgl. Van de Ven, A. Delbecq, A., Koenig R. Jr., Determinants of Coordination Modes within Organizations, in: American Sociological Review, Vol. 41, 1976, S. 329

3 Emeut wird die Benutzung der Begriffe horizontal und vertikal vermieden, um eine (Fußnote wird auf der nächsten Seite fortgesetzt) 
plante Zusammentreffen von Teams (unscheduled, scheduled Meetings). ${ }^{1}$ Dabei wird eindeutig und konsistent der vermutete Zusammenhang zwischen dem Einsatz der Koordinationsmechanismen und der Unsicherheit bestätigt.

Während die administrativen Koordinationsmechanismen mit steigender Unsicherheit an Bedeutung verlieren, nehmen die strukturellen Koordinationsmechanismen an Bedeutung leicht $\mathrm{zu}$, während die personalen Koordinationsmechanismen bzw. hier der Einsatz von Teams erheblich zunimmt (vgl. Abb. B.2.22).
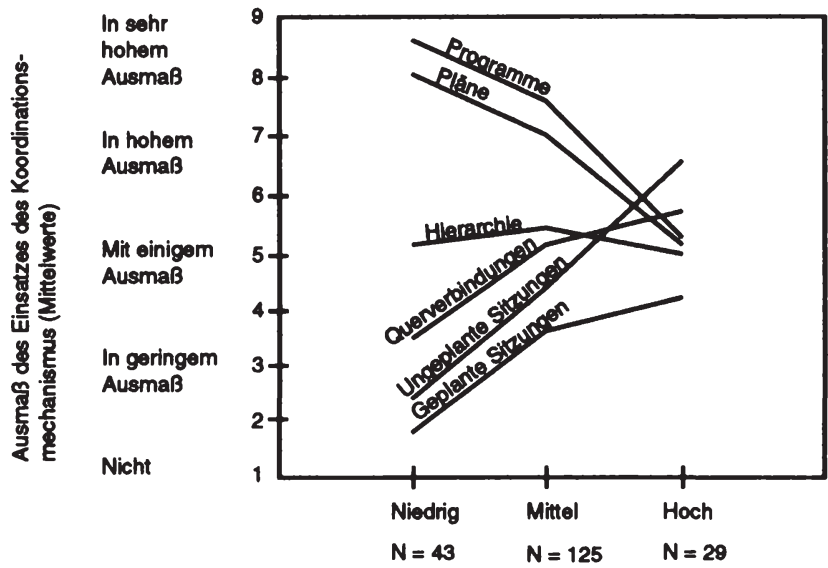

Klassifizierte Unsicherheit der Autgabe

Abb. B. 2.22: Profil des Einsatzes von Koordinationsmechanismen in Abhăngigkeit von der Unsicherheit der Aufgabe

Quelle: Van de Ven, A. Delbecq, A., Koenig R. Jr., Determinants of Coordination Modes within Organizations, in: American Sociological Review, a.a.O., S. 329.

Verwechslung mit den Begriffen horizontalen Verflechtungen und vertikalen Verknüpfungen auszuschließen. Zum Begriff horizontal und vertikal vgl. Abschnitt B.2.1.2.

1 Vgl. zum folgenden Van de Ven, A., Delbecq, A., Koenig, R.Jr., Coordination Modes, a.a.O., insbesondere S. $324 \mathrm{ff}$. 
Nach Van de Ven, Delbecq und Koenig werden die administrativen und strukturellen Koordinationsmechanismen in gewissem Umfang nahezu immer eingesetzt, während Teams bei niedriger Unsicherheit nur geringe Bedeutung haben. Dies entspricht den Ergebnissen der Studie von Lawrence und Lorsch, die den situativen Ansatz ${ }^{1}$ der Organisationstheorie mitbegründet.hat. Die beiden Autoren fanden die grundlegenden administrativen und strukturellen Koordinationsmechanismen in nahezu jeder Umweltsituation vor. ${ }^{2}$ Mit wachsender Unsicherheit werden zunehmend aufwendige strukturelle und personale Koordinationsmechanismen eingesetzt, wie aus dem Vergleich erfolgreicher Unternehmen in Branchen mehr oder minder großer Unsicherheit hervorgeht.

Auch die Untersuchungsergebnisse von Burns und Stalker sind mit diesen Aussagen konsistent. Burns und Stalker verglichen den Führungsstil von Organisationen in unterschiedlichen Umfeldern, und zwar stabilen (d.h. geringe Unsicherheit) und sich wandelnden (d.h. hohe Unsicherheit). Sie fanden in stabilen Umfeldern eine hohes Ausmaß von administrativen Koordinationsmechanismen vor, z.B. detaillierte Arbeitsplatzbeschreibungen. In sich stark wandelnden Umfeldern fanden sie ein hohes Ausmaß an personalen Koordinationsmechanismen vor, z.B. informelle Teamkommunikation. Sie leiten daraus die Unterscheidung von mechanistisch und organisch geführten Organisationen ab. ${ }^{3}$

1 Lawrence, P., Lorsch, J., Organization and Environment, a.a.O., S. 185 ff. Der Ansatz geht zurück auf Einen Überblick über deutsche situative Ansätze gibt Staehle, W., Deutschsprachige situative Ansätze in der Managementlehre, in: Wirtschaftswissenschaftliches Studium, München, 1979, S. 218 - 222.

2 Zum folgenden vgl. Lawrence, P., Lorsch, J., Organization and Environment, , a.a.O., S. 131 ff.

3 Vgl. Burns, T., Stalker, G.M., Management of Innovation, a.a.O., S. 83 ff. 


\subsubsection{Kontrakte und situative Bedingungen}

Je höher die Spezifität der Geschäftsaktivitäten im Integrationsbereich, desto eher werden relationale und Unternehmenskontrakte eingesetzt, um eine hohe Integrationsintensität der vertikalen Verknüpfungen und horizontalen Verflechtungen zu erreichen, insbesondere bei gleichzeitig hohem Volumen und großer Unsicherheit. ${ }^{1}$ Diese Basishypothese des Transaktionskostenansatzes wird mit der komparativen Vorteilhaftigkeit alternativer kontraktueller Arrangements, den Transaktionskosten, begründet. Eher marktbezogene Kontrakte weisen vergleichsweise bessere Anreizmechanismen auf, während eher unternehmenskonstituierende Kontrakte vergleichsweise bessere administrative Kontrollmöglichkeiten bieten. ${ }^{2}$ Der Tausch von Leistungen am Markt ist daher solange überlegen, wie keine Investitionen in spezifische Faktoren vorgenommen werden müssen. Sind solche Commitments jedoch erforderlich, eröffnen Marktkontrakte die Möglichkeit der Abschöpfung von Rente. Demgegenüber können Commitments durch kontraktuelle Integration abgesichert werden: In relationalen Kontrakten werden langfristige Vereinbarungen über die Nutzung der spezifischen Faktoren getroffen und etwaiger Anpassungsbedarf durch Regeln prädisponiert. Unternehmenskonstituierende Kontrakte bewirken die kontraktuelle Integration in die Unternehmung, die ein Commitment eingeht, so daß die etwaige Abschöpfung von Rente unproblematisch wird.

Damit hängt die Art des Kontraktes von der Faktorspezifität ab. Dies bildet Williamson wie folgt $\mathbf{a b}^{3}$ In Abhängigkeit von der Faktorspezifität $\mathbf{k}$ werden die Kosten des Tauschs am Markt, $\mathbf{M}=\mathbf{M}(\mathbf{k})$, die Kosten der Hierarchie, $H=H(k)$ und die Kosten der relationalen Verträge, $X=X(k)$ abgetragen (vgl. Abb. B.2.23).

1 Vgl. Klein, B., Crawford, R., Alchian, A.A., Vertical Integration, a.a.O., S. 298 ff; Williamson, O.E., Economic Institutions, a.a.O., S. 52 ff.

2 Vgl. z.B. Williamson, O.E., Economic Organization, a.a.O., S. 32.

3 Vgl. Williamson, O.E., Economic Organization, a.a.O., S. 22 ff. 


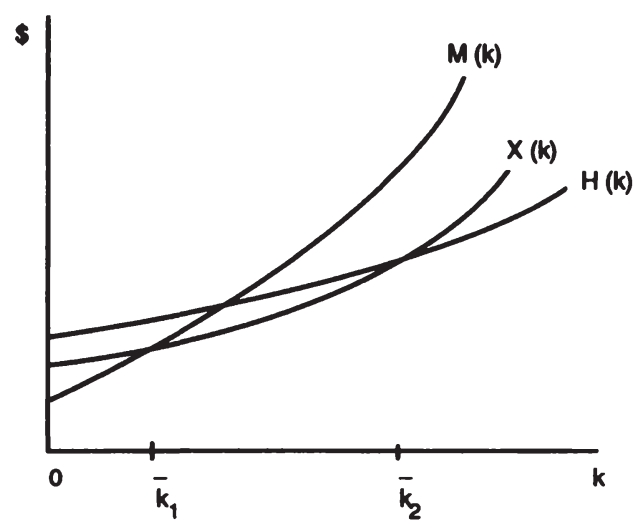

Abb. B.2.23: Spezifitat als Bestimmungefaltor der Kontraktgestaltung

Quelle: Williamson, O.E., Comparativ e Economic Organization, in: Botriebswintschaftslohre und okonomische Theorie, Ordelheide, D., et al., Hreg., Stuttgart 1991, S. 24

Ohne Faktorspezifität ist der Markt günstiger als relationale Verträge und unternehmenskonstituierende Kontrakte. Mit zunehmender Faktorspezifität wachsen jedoch die bei Markttausch drohenden Abschöpfungsrisiken. Auch die Kosten relationaler Verträge und der Hierarchie wachsen mit der Faktorspezifität, jedoch geringer als $\mathrm{M}(\mathbf{k})$. Insgesamt ergeben sich drei Intervalle der komparativen Vorteilhaftigkeit jeweils eines kontraktuellen Arrangements über beide anderen: bis $k 1$ der Marktkontrakt, zwischen $k 1$ und $\mathbf{k} 2$ die relationalen Kontrakte und über $\mathbf{k} 2$ die Unternehmung, d.h. das (Allein-)Eigentum der spezifischen Produktionsfaktoren durch denjenigen, für den sie spezifisch sind. Subsidiär sind auch die beiden anderen situativen Faktoren im Integrationsbereich heranzuziehen, das Volumen und die Unsicherheit. Dabei gilt tendenziell, daß je höher Volumen und Unsicherheit sind, desto weiter verschiebt sich die Vorteilhaftigkeit hin zu unternehmenskonstituierenden Kontrakten. Dies kann beim Volumen mit Skaleneffekten begründet werden, die ein aufwendigeres kontraktuelles Arrangement (Unternehmung) c.p. günstiger machen können. Mit wachsender Unsicherheit werden unternehmenskonstituierende Kontrakte günsti- 
ger, da sie die Anpassung an unsichere Zukunftslagen bei Existenz von spezifischen Faktoren ermöglichen.

\subsection{Nutzen und Kosten der Integration}

Mit den drei Kontinua von Integrationsinstrumenten wurde ein Möglichkeitsraum von Handlungsalternativen aufgespannt. Kriterien für die Wahl der Integrationsinstrumente sind der Nutzen und die Kosten der Integration. (vgl. Abb. B.2.24). Der optimale Grad der Integration ist erreicht, wenn Grenznutzen und Grenzkosten der Integration ausgeglichen sind. Deswegen ist im folgenden, zunächst in allgemeiner Formulierung, die (Grenz)Nutzenkurve und die (Grenz-)Kostenkurve der Integration von Geschäftsaktivitäten $\mathrm{zu}$ entwickeln.

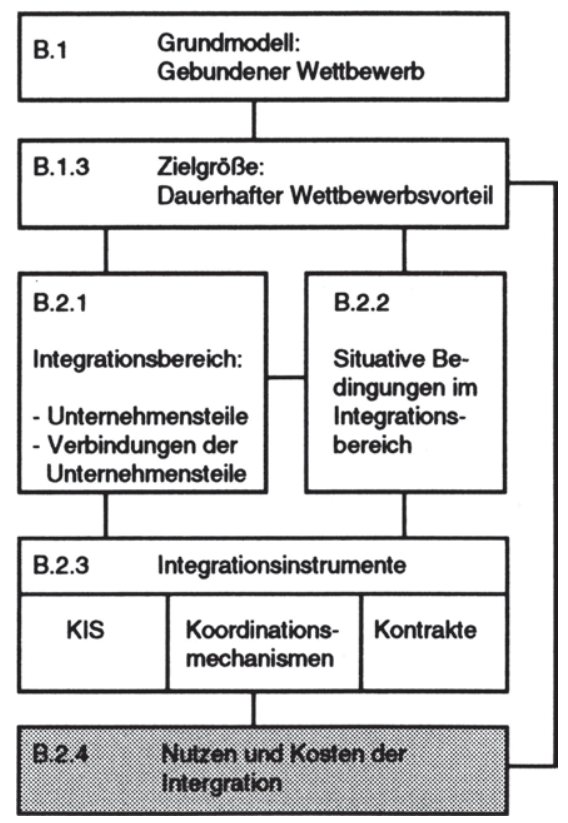

Abb. B.2.24: Stellung von Nutzen und Kosten im Bezugsrahmen 


\subsubsection{Nutzen der Integration}

Der Nutzen der Integration ist ihr Beitrag zum Unternehmensziel, hier operationalisiert als dauerhafter Wettbewerbsvorteil. Die Unternehmung wurde als zielgerichtetes ${ }^{1}$, offenes, sozio-technisches System der Güter- und Informationstransformation ${ }^{2}$ definiert. Das Unternehmensziel wird erreicht durch Güter- und Informationstransformation, die wiederum auf Spezialisierung und Integration beruht. ${ }^{3}$ Spezialisierung schafft Wert durch die Nutzung komparativer Vorteile von Wirtschaftssubjekten, die einen beiderseitig vorteilhaften Tausch ermöglichen. ${ }^{4}$ Der Wert der Spezialisierung ergibt sich aus der Größe der durch sie geschaffenen komparativen Vorteile (z.B. durch Skaleneffekte). Durch Integration werden die spezialisierten Faktoren so kombiniert, daß aus ihrem Zusammenwirken in einem Bündel von Faktoren synergetischer Wert entsteht. Soll eine Ganzheit mehr wert sein als ihre Teile, kann das nur auf die Art der Verbindung der Teile zurückgeführt werden. ${ }^{5}$ Dabei wurde als Maßstab des Zielbeitrags strategischer Integrationsentscheidungen deren Beitrag zum dauerhaften Wett-

1 Barnard, Ch., The Functions of the Executive, Neudruck, Cambridge, Massachusetts, 1938, S. 86.

2 Vgl. Meffert, H., Informationssysteme, a.a.O., S. 2 und Meffert, H., Marketing, a.a.O., S. 23.

3 Vgl. z.B. Nicklisch, H, Organisation, 2. Aufl., Stuttgart 1922, S.77; Kubicek, H., Informationstechnologie und organisatorische Regelungen, a.a.O., S. 50 ff; Kosiol, E., Organisation der Unternehmung, Wiesbaden, 1962, S. 21.

4 Vgl. z.B. Alchian, A.A., Demsetz, H., Production, a.a.O., S. 779 ff.

5 Vgl. Nagel, E., On the statement: "The whole is more than the sum of its parts", in: The Language of Social Research, (Hrsg.), Lazarsfeld, P., Rosenberg, M., Glencoe, Illinois, 1955, wiederabgedruckt als: Über die Aussage: "Das Ganze ist mehr als die Summe seiner Teile", in: Logik der Sozialwissenschaften, (Hrsg.), Topitsch, F. von, Köln, Berlin, 1965, S. 225 - 235; Schlick, M., Über den Begriff der Ganzheit, in: Moritz Schlick, Gesammelte Aufsätze, Wien, 1938, wiederabgedruckt in: Logik der Sozialwissenschaften, (Hrsg.), Topitsch, F. von, Köln, Berlin, 1965, S. 213 - 224. Nagel zeigt den Zusammenhang der Interdependenzmodi mit den Beziehungen von Elementen in Systemen am Beispiel physikalischer Systeme auf. Vgl. Nagel, E., On the Statement: The whole is more, a.a.O., 1955, S. 234 ff. Konsistent dazu haben Lawrence und Lorsch empirisch nachgewiesen, daß der Nutzen (Gewinnbeitrag) von Koordinationsmechanismen um so größer ist, je stärker die Interdependenzen zwischen den organisational integrierten Unternehmensteilen sind. Lawrence, P.R., Lorsch, J.W., Organizations, a.a.O., S. 137 ff. 
bewerbsvorteil gewählt, untergliedert in Qualitätsvorteil, Kostenvorteil, Zeitvorteil, Stabilitätsvorteil, Flexibilitätsvorteil und Verteilungsvorteil.1

Den Verlauf des Nutzens aus Integration illustriert Abb. B.2.26 schematisch in Abhängigkeit vom Integrationsgrad. ${ }^{2}$ Würde nicht integriert, läge kein Nutzen aus Integration vor. Mit steigendem Integrationsgrad wird der Nutzen aus Integration zunächst rasch zunehmen, wobei der Grenznutzen der Integration mit zunehmendem Integrationsgrad abnimmt. Sein Maximum $\mathbf{N}$ wird der Nutzen der Integration bei einem geringeren Integrationsgrad als der "totalen Integration" annehmen, und zwar aus vier Gründen: Komplexitätsprobleme, Flexibilitätsverluste, Fehlerakkumulation und Kompromißpositionen.

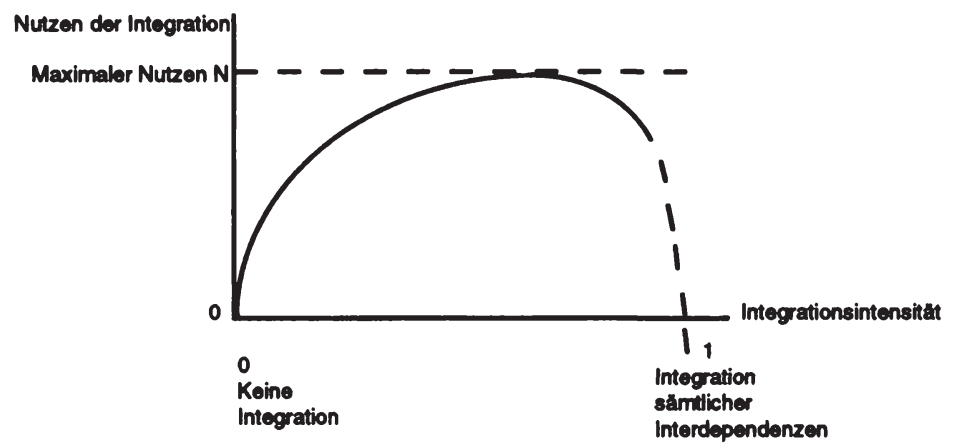

B.2.25: Nutzen der Integration

Komplexitätsprobleme ergeben sich aus der Berücksichtigung sämtlicher Interdependenzen. Dies verlängert und kompliziert Entscheidungsprozesse in

1 Vgl. Abschnitt B.1.3.1

2 Dies geschieht lediglich zur allgemeinen Definition eines Zustandes optimaler Integration im Bewußtsein, daß es unmöglich ist, einen solchen Nutzenwert für eine Unternehmung genau anzugeben. Falls die Unternehmensteile jedoch einen Marktpreis oder einen schätzbaren Veräußerungswert haben, ist die Ermittlung der Differenz am Unternehmenswert trivial. Vgl. Copeland, T. Koller, T., Murrin, J., Valuation, a.a.O., 1990, Kapitel 9. Hier werden jedoch stark intendependente Unternehmensteile diskutiert, für die solche Werte entweder kaum ermittelbar sind, oder aufgrund der Zerschneidung von wichtigen Interdependenzen eine Veräußerung nicht in Betracht kommt. 
der Unternehmung und verschlechtert Entscheidungen im Hinblick auf Inhalt und Zeitbedarf. ${ }^{1}$ Flexibilitätsverluste entstehen, weil Integration die partielle Starrheit eines Faktorbündels mitbegründet und insoweit im Zeitablauf nicht beliebig änderbar ist. Sind zwei Unternehmensteile integriert und muß sich einer aufgrund wettbewerblicher Erfordernisse verändern, stellt eine enge Integration mit einem sich nicht verändernden Unternehmensteil ein Flexibilitätshindernis dar. Zudem mag sich das gemeinsame Ausführen einer Geschäftsaktivität durch verschiedene Unternehmensteile als Marktaustrittsbarriere für einen Unternehmensteil erweisen, somit die Flexibilität der strategischen Position reduzieren. ${ }^{2}$ Ein erhebliches Risiko totaler Integration ist die Fehlerakkumulation. Je enger z.B. ein Produktionssystem verknüpft ist, desto anfälliger reagiert es auf Abweichungen. Werden an einer Stelle falsche Daten eingegeben, wird dieser Fehler auf allen Produktionsstufen bis hin zur Distribution multipliziert. Bei Mehrfacheingabe wäre der Fehler auf den Ort der Fehleingabe begrenzt geblieben. ${ }^{3}$ Darüber hinaus kann (totale) Integration Kompromißpositionen nach sich ziehen. So kann die gemeinsame Nutzung eines Distributionsnetzes durch verschiedene Produktgruppen dazu führen, daß weder die technischen noch die personellen Voraussetzungen für jedes einzelne Produkt vorliegen, so daß Verluste aus suboptimaler Kundenbetreuung und suboptimalem Distributionshandling entstehen.4 Damit gehen segmentspezifische Differenzierungsmöglichkeiten verloren.

\subsubsection{Kosten der Integration}

Integration ist nicht kostenlos. Der Verlauf der Integrationskostenkurve in Abhängigkeit vom Integrationsgrad kann aus den Arten der Integrationskosten abgeleitet werden. Unter den Integrationskosten seien die Auto-

1 Vgl. Emery, J.C., Management Information Systems, a.a.O., S. 253.

2 Vgl. Porter, M.E., Competitive Advantage, a.a.O., S. 334.

3 Vgl. Emery, J.C., Management Information Systems, a.a.O., S. 253.

4 Vgl. Porter, M.E., Competitive Advantage, a.a.O., S. 333 ff. 
nomiekosten ${ }^{1}$ und die Agencykosten ${ }^{2}$ unterschieden. Autonomiekosten entstehen aus der (opportunistischen) Verfolgung von Subzielen und Partikularinteressen in Unternehmensteilen. Agencykosten bilden das Gegenteil; sie entstehen durch nachlässiges Verhalten bei Integration und den administrativen Aufwand der Integration angesichts begrenzter Rationalität. ${ }^{3}$

\subsubsection{Autonomiekosten}

Durch den Einsatz von Integrationsinstrumenten, nämlich KIS, Koordinationsmechanismen und Kontrakten, werden Unternehmensteile so verbunden, daß sich Zielfunktion, Willensbildung und Informationsverwendung auf eine gemeinsame höhere Ebene verlagern. Ließe die Unternehmung demgegenüber die Autonomie der Unternehmensteile zu, könnten jene ungehindert ihre jeweiligen Subziele und "Partikularinteressen" verfolgen, d.h. sie könnten die persönlichen Ziele den Zielen der Unternehmung voranstellen.4 Dadurch entstünden der Unternehmung Kosten, Autonomiekosten. Je stärker die Unternehmensteile durch rechtliche, technische und organisationale Integration in das gemeinsame System eingebun-

1 Vgl. Emery, J.C., Organizational Planning and Control: Theory and Technology, London, 1969, S. 29 ff. Eine deutsche Darstellung gibt Frese, E., Organisation, a.a.O., S. 210 f.

2 Vgl. Pratt, J.W., Zeckhauser, R.J., (Hrsg.), Principals and Agents: The Structure of Business, Boston, Massachusetts, 1985, S. 3.

3 Wirtschaftliches Handeln wird als im Durchschnitt begrenzt rational und opportunistisch angenommen. Begrenzte Rationalitzit ist ein Anstreben rationalen Handelns, jedoch beschränkt durch die kognitiven Grenzen des Menschen, Entscheidungsalternativen vollständig zu erfassen und au bewerten. Vgl. Simon, H.A., Models of Man: New York, 1957, S. 198; ders., Administrative Behavior, a.a.O., S. XXIV, 38 ff und 75 ff; Heinen, E., Zum Wissenschaftsprogramm der entscheidungsorientierten Betriebswirtschaftslehre, in: Zeitschrift für Betriebswirtschaft, 1969, S. 215. Der Begriff der begrenzten Rationalität verbindet die neoklassische Annahme des Strebens nach rationalen Entscheidungen, d.h. maximaler Zielerreichung, mit dem Rationalitätspessimismus verhaltenswissenschaftlicher und evolutorischer Ansätze. Williamson, O.E., Economic Institutions, a.a.O., S. 44 ff. Opportunismus ist ein Verfolgen des Selbstinteresses auch unter Einsatz fragwürdiger Mittel. Vgl. Williamson, O.E., Economic Institutions, a.a.O., S. 47 ff. Die Möglichkeit opportunistischen Verhaltens ist bei der Ausgestaltung integrativer Beziehungen von Unternehmensteilen an berücksichtigen, bei hohem Integrationsgrad in Form von Nachlässigkeitskosten, bei schwachem Integrationsgrad in Form von Autonomiekosten.

4 Zur Unterscheidung dieser Zielebenen vgl. Kieser, A., Kubicek, H., Organisation, a.a.O., (Fußnote wird auf der nächsten Seite fortgesetzt) 
den werden, desto weniger wird ihnen die opportunistische Verfolgung von Subzielen möglich sein. Die Autonomiekosten sinken also mit steigendem Integrationsgrad.1

\subsubsection{Agencykosten}

Demgegenüber steigen die Agencykosten mit steigendem Integrationsgrad. Durch die Verschiebung der Zielfunktion, der Willensbildung und der Informationsverwendung auf eine höhere Ebene des Systems wird das Prinzipal-Agenten-Problem verschärft: Maßnahmen werden auf einer übergeordneten Ebene beurteilt, jedoch in der untergeordneten Ebene ausgeführt. ${ }^{2}$ Dies verursacht zwei Arten von Kosten: die Nachlässigkeitskosten und die Administrationskosten. ${ }^{3}$ Nachlässigkeitsprobleme entstehen, weil sich die Verantwortung eines integrierten Unternehmensteils auf einen schwer meßbaren Beitrag zum Ganzen bezieht, während der autonome Unter-

\section{S. 2 f.}

1 Zum gleichen Schluß kommt auch Emery, J.C., Organizational Planning, a.a.O., und nach ihm Frese, wobei Emery die Autonomiekosten als Differenz zwischen einem theoretisch denkbaren Optimum und dem tatsächlich realisierten Engebnis definiert. Insofern verwenden Emery und Frese die Summe aus den hier definierten Autonomiekosten und dem umgekehrt genommenen Integrationsnutzen als Autonomiekosten. Dabei entfällt jedoch die Möglichkeit, beide analytisch verschiedenen Kategorien auch verschieden zu analysieren, beispielsweise die Wurzel negativen Integrationsnutzens zu erkennen, den Integrationsnutzen in Teildimensionen aufzuspalten sowie Integrationsnutzen und Autonomiekosten bei verschiedenen Wirkungsverläufen getrennt abzubilden. Dies ist allerdings auch nicht das Erklärungsziel von Emery bzw. Frese. Vgl. Frese, E., Organisation, a.a.O., S. 210 f.

2 Vgl. Pratt, J.W., Zeckhauser, R.J., (Hrsg.), Principals and Agents, a.a.O., S. 2 f; Glossman, S.J., Hart, O.D., Ownership, a.a.O., S. 716. Die Verlagerung auf eine höhere Ebene muß dabei nicht bedeuten, daß Entscheidungen auf einer höheren Hierarchieebene getroffen werden, vielmehr ist der gemeinsame ProzeB der Entscheidungsfindung bzw. Abstimmung zweier oder mehrerer Unternehmensteile als "höhere Ebene"-Prinzipal anzusehen, dessen Agenten die einzelnen Unternehmensteile sind.

3 McFarlan und Bruns unterscheiden analog das "Don't want to do it"-Problem und das "Don't know what to do"-Problem, Arrow die Probleme von "Hidden Action" und "Hidden Information". Vgl. Arrow, K.J., The Economics of Agency, in: Principals and Agents; The Structure of Business, (Hrsg.), Pratt, J.W., Zeckhauser, R.J., Boston, Massachusetts, 1985, S. 28; McFarlan, F.W., Brunns, W., How Information Technology is Changing Management Control Systems, Boston, Massachusetts, Harvard Business School, Note, No. 9-187-137, 1987, S. 5. 
nehmensteil die volle Verantwortung eigenen Handelns trägt. ${ }^{1}$ Damit ist sowohl der Einfluß des integrierten Unternehmensteils auf die Ausbringung geringer als auch der spezifische Beitrag des integrierten Unternehmensteils schwieriger zu ermitteln. ${ }^{2}$ Aufgrund höherer Nachlässigkeit und geringerer Bedeutung von suboptimalem Verhalten wird Integration eine geringere Produktivität (shirking) nach sich ziehen, also Nachlässigkeitskosten verursachen. ${ }^{3}$ Milgrom diskutiert auch indirekte Nachlässigkeitskosten, die "Einflußkosten" (Influence Costs). Mitarbeiter haben Einfluß auf ihre Vorgesetzten. Diesen Einfluß können sie nutzen, um unerwünschte Aktivitäten durchzusetzen, die nicht der Unternehmung, sondern nur dem betreffenden Mitarbeiter zu gute kommen.4 Nachlässigkeitskosten sind das Umkehrproblem der Autonomiekosten. Während Autonomiekosten durch die Verfolgung von Subzielen anfallen, also durch hohen Arbeitseinsatz für die "falschen Ziele", werden Nachlässigkeitskosten durch das "Untergehen im großen Topf"5 verursacht, also geringen Arbeitseinsatz für die "richtigen Ziele".6

Der zweite Bestandteil der Agencykosten sind die Administrationskosten, bestehend aus Informationskosten sowie Meß- und Kontrollkosten. Die Administrationskosten sind insoweit die Kosten für den Einsatz der Integrationsinstrumente selbst. Die Informationskosten steigen durch den

1 Vgl. Laux, H., Anreizsysteme, a.a.O., S. 201.

2 Vgl. Pratt, J.W., Zeckhauser, R.J., (Hrsg.), Principals and Agents, a.a.O., S. 8; Klein, B., Crawford, R., Alchian, A.A., Vertical Integration, a.a.O., S. 325; Hart, O.D., Incomplete Contracts, a.a.O., S. 119; Alchian, A.A., Demsetz, H., Production, a.a.O., S. 779; Sie stellen dies als nicht separables Gemeinschaftsprodukt $Z$ dar, das nicht in Grenzprodukte $Z_{i}$ und $Z_{j}$ separiert werden kann, die auf die Inputs $X_{i}$ und $X_{j}$ zurückführbar sind. Dies gilt, wern für die Produktion von $Z\left(\partial^{2} Z / \partial X_{i} \partial X_{j}\right) \neq 0$ (andernfalls ist die Produktionsfunktion separabel und der isolierte Beitrag jedes Faktors ablesbar).

3 Vgl. Pratt, J.W., Zeckhauser, R.J., (Hrsg.), Principals and Agents, a.a.O., 1989, S. 9, Alchian, A.A., Demsetz, H., Production, a.a.O., 1972, S. 777 ff.

4 Vgl. Milgrom, P., Employment Contracts, Influence Activities, and Efficient Organizational Design, in: Journal of Political Economy, Vol. 96, 1988, S. 42 - 60.

5 Vgl. Hardin, G., The Tragedy of the Commons, in: Science, Vol. 162, No. 3859, December 13,1968, S. 1243.

6 Dieses Argument geht zurück auf Grossman, S.J., Hart, O.D., Ownership, a.a.O., S. 691. Vgl. auch Laux, H., Anreizsysteme, a.a.O., S. 201. 
wachsenden Bedarf, Informationen zu generieren und auszutauschen, je mehr Interdependenzen berücksichtigt werden sollen ${ }^{1}$ und je höher der angestrebte Integrationsnutzen ist. ${ }^{2}$ Der Integrationsnutzen kann z.B. als eine höhere Kapazitätsauslastung spezifiziert werden, d.h. je höher die angestrebte Kapazitätsauslastung desto höher die Informationskosten. ${ }^{3}$ Zur Ausschöpfung vertikaler Verflechtungen müssen Informationen darüber ausgetauscht werden, welche Spezifikation ein Zwischenprodukt haben muß, um in die nächste Stufe einzugehen. Es müssen laufend Informationen über Terminierung und Arbeitsfortschritt erhoben und übermittelt werden. Zur Ausschöpfung horizontaler Verflechtungen müssen Informationen ausgetauscht werden, um die Probleme bei der Nutzung gemeinsamer Produktionsfaktoren abzustimmen, also die Faktorallokation 4 , Nutzungsterminierung, Prioritätensetzung, Bestimmung der Qualität und Art der gemeinsam zu nutzenden Faktoren ${ }^{5}$ etc.

Für die Administration einer Organisation fallen nicht nur Kosten des Informationsaustauschs an. Organisationen werden nicht nur durch den Austausch von Informationen integriert, sondern auch durch die Willensdurchsetzung, Steuerung und Kontrolle. Dabei bestehen zwei Grundalter-

1 Vgl. Frese, E., Organisation, a.a.O., S. 210.

2 Galbraith, J.R., Complex Organizations, a.a.O., S. 5.

3 Galbraith, J.R., Complex Organizations, a.a.O., S. 5. Dies folgt auch aus Lawrence, P.R., Lorsch, J.W. Organizations, a.a.O., S. 133 ff. Angesichts starker Interdependenzen nehmen erfolgreiche Unternehmen (operationalisiert am Gewinniveau) höhere Administrationskosten auf sich als a) weniger erfolgreiche Unternehmen und b) erfolgreiche Unternehmen mit geringeren Interdependenzen.

4 Zur Identifikation des theoretischen Optimums müßten die wertmäßigen Kosten (Schattenpreise) der Ressource ermittelt werden. Dies wäre nur möglich, wenn vollständige Informationen über die Knappheitssituation und die alternativen Verwendungsmöglichkeiten vorlägen. Schon ein partielles, heuristisches Ermitteln dieser Informationen wird hohe Informationskosten verursachen. Zum Konzept der wertmäßigen Kosten vgl. Adam, D., Entscheidungsorientierte Kostenbewertung, Wiesbaden, 1970, S. 33 92.

5 An diesem Beispiel kann erneut verdeutlicht werden, wie die Kosten der Integration und der negative Nutzen der Integration in dieser Arbeit unterschieden werden. Ein Produktionsproze $B$ werde für die Herstellung von Spezialteilen für Automobile verschiedener Baureihen genutzt. Informationskosten engeben sich aus dem Abstimmungsbedarf über Terminierung, Allokation etc. Negativer Nutzen ergibt sich aus der Kompromißposition, z.B. ist die Autoelektrik fast aller Marken von General Motors identisch, was im hochpreisigen Segment Imageprobleme nach sich ziehen dürfte. 
nativen: Transfer der Informationen oder der Beurteilungskriterien. Das Prinzipal-Agenten-Problem entsteht durch das Auseinanderfallen von Beurteilungsebene und Ausführungsebene. Notwendige Informationen liegen auf der Ausführungsebene vor, die Beurteilungskriterien jedoch auf der Beurteilungsebene. Informationsaustausch mindert das Problem, indem die auf der Ausführungsebene vorhandenen Informationen (nach "oben") auf die Beurteilungsebene transferiert werden. Im Gegensatz dazu mindern Steuerungs- und Kontrollmechanismen das Problem, indem die Beurteilungskriterien (nach "unten) von der Beurteilungsebene auf die Ausführungsebene übermittelt werden. ${ }^{1}$ Dafür fallen Steuerungskosten an. Entweder werden auf der Ausführungsebene Vorgaben definiert und deren Einhaltung überprüft oder die Ausführungsebene wird durch das Setzen von Anreizen gelenkt. ${ }^{2}$ Anreize, z.B. erfolgsabhängige Entlohnung, sollen dazu beitragen, die "Ziele für die Unternehmung" und die "persönlichen Ziele" auf die "Ziele der Unternehmung" auszurichten. Weder das Setzen von Anreizen noch die Definition und Kontrolle von Vorgaben ist kostenlos. ${ }^{3}$ Anreize sind nicht kostenlos, weil z.B. bei erfolgsabhängiger Entlohnung für den Empfänger aus einer sicheren Entlohnung eine ungewisse Entlohnung wird. Je höher die erwartete Varianz und je höher die Risikoaversion des Empfängers ist, desto höher ist die zu zahlende Risikoprämie.4 Die Steuerung durch Definition und Kontrolle von Vorgaben ist angesichts begrenzter Rationalität ebenfalls nicht kostenlos, insbesondere bei starken Interdependenzen und heterogener, dynamischer Umwelt. ${ }^{5}$ Problematisch

1 Vgl. Jensen, M.C., Meckling, W.H., Coondination, Control and the Management of Organizations, Vorlesungsskript, Boston, Massachusetts, Harvard Business School, 1988, S. 15.

2 Vgl. Laux, H., Anreizsysteme, a.a.O., S. 201.

3 Laux, H., Anreizsysteme, a.a.O., S. 201.

4 Vgl. Laux, H., (Pareto-)Optimale Anreizsysteme bei unsicheren Erwartungen, in: Zeitschrift für Betriebswirtschaftliche Forschung, 40. Jg., 1988, S. 1093 - 1111. Das Set von den hinter dieser Aussagen stehenden Annahmen wird auch angegeben bei Laux, H., Anreizsysteme, a.a.O., S. 202 - 203. Das Argument ist auch auf andere Anreizformen, z.B. Beförderungsmöglichkeiten, ausdehnbar.

Galbraith, J.R., Complex Organizations, a.a.O., S. 5. 
(bzw. aufwendig) ist in diesem Zusammenhang die Definition, Messung und Bewertung der Leistungen des einzelnen Unternehmensteils. ${ }^{1}$

Zwischen den Arten der diskutierten Agencykosten bestehen vielerlei Beziehungen, z.B. Zielkonflikte zwischen Administrations- und Nachlässigkeitskosten, Informations- und Steuerungskosten sowie Anreiz- und Kontrollkosten. Welcher Integrationsgrad in Bezug auf die Agencykosten vorteilhaft ist, kann nur im Lichte einer konkreten Situation beurteilt werden; als analytische Basis wird die diskutierte Systematik der Kostenarten der Integration vorgeschlagen.

Insgesamt werden sich die Agencykosten bei wachsenden Integrationsgraden durch die Vergrößerung des Prinzipal-Agenten-Problems erhöhen, da jede einzelne Agencykostenart bei stärkerer Integration steigt. Demgegenüber sinken die Autonomiekosten bei höheren Integrationsgraden. Eine schematische Darstellung des gegenläufigen

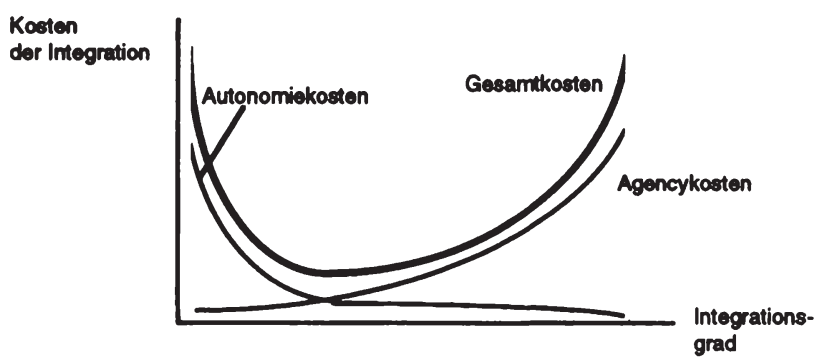

Abb. B.2.26: Kosten der Integration

Verlaufs der beiden Kostenarten und der Gesamtkosten zeigt Abb. B.2.26.

1 Barzel, Y., Measurement Cost and the Organization of Markets, in: Journal of Law and Economics, Vol. 25, 1982, S. 27 ff; Williamson, O.E.; Economic Institutions, a.a.O., S. 212; Laux, H., Anreizsysteme, a.a.O., S. 201. Die Kontrollkosten korrespondieren mit den Nachlässigkeitskosten. Bei prohibitiv hohen Kontrollkosten unterbleibt die vollständige Definition, Messung und Kontrolle von Leistungen, wodurch Nachlässigkeitskosten überhaupt erst entstehen. Dieser Zusammenhang wird zusammengefaßt bei Pratt, J.W., Zeckhauser, R.J., (Hrsg.), Principals and Agents, a.a.O., S. 8 ff. 


\subsubsection{Optimaler Integrationsgrad}

Der Integrationsgrad ist das $\mathrm{Maß}$, in dem Interdependenzen zwischen den die Geschäftsaktivitäten tragenden Unternehmensteilen durch integrative Verbindungen im Integrationsbereich berücksichtigt werden. Auf Kosten und Nutzen der Integration aufbauend, kann ein optimaler Integrationsgrad definiert werden: Der optimale Integrationsgrad $i$ ist der Integrationsgrad, bei dem der Überschuß des Integrationsnutzens über den Integrationskosten maximal ist. ${ }^{1}$ In Abb. B.2.27 sind außer den Gesamtkostenkurven die jeweiligen Grenzkostenkurven abgetragen. Ihr Schnittpunkt kennzeichnet die optimale Integrationsintensität i.

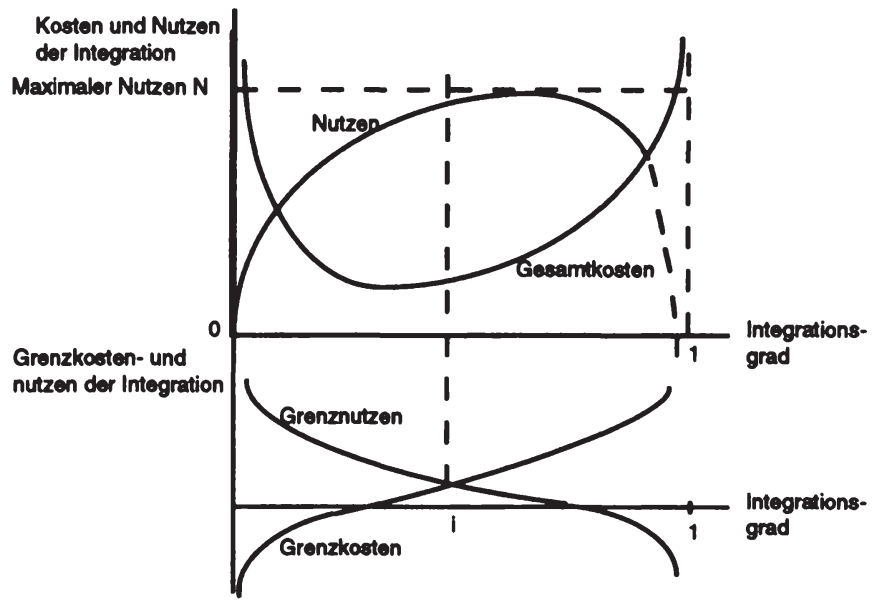

Abb. B.2.27: Optimale Integration

1 Eine ähnliche Darstellung von Gesamtkosten und Gesamtnutzen der technischen Integration von Daten gibt Scheer, A.-W., EDV-orientierte Betriebswirtschaftslehre, a.a.O., S. 46. 
Ein Anwendungsbeispiel für diesen Ansatz ist die Untersuchung der Frage, wie sich der verstärkte Einsatz von neuen KIS auf den optimalen Integrationsgrad von Geschäftsaktivitäten auswirken wird. Aus den vorangegangenen Kapiteln ist zu schließen, das die erhebliche Bedeutung der KIS für das Integrationsmanagement darauf zurückgeführt werden kann, daß sich sowohl die Kosten als auch der Nutzen der Integration durch KIS-Einsatz

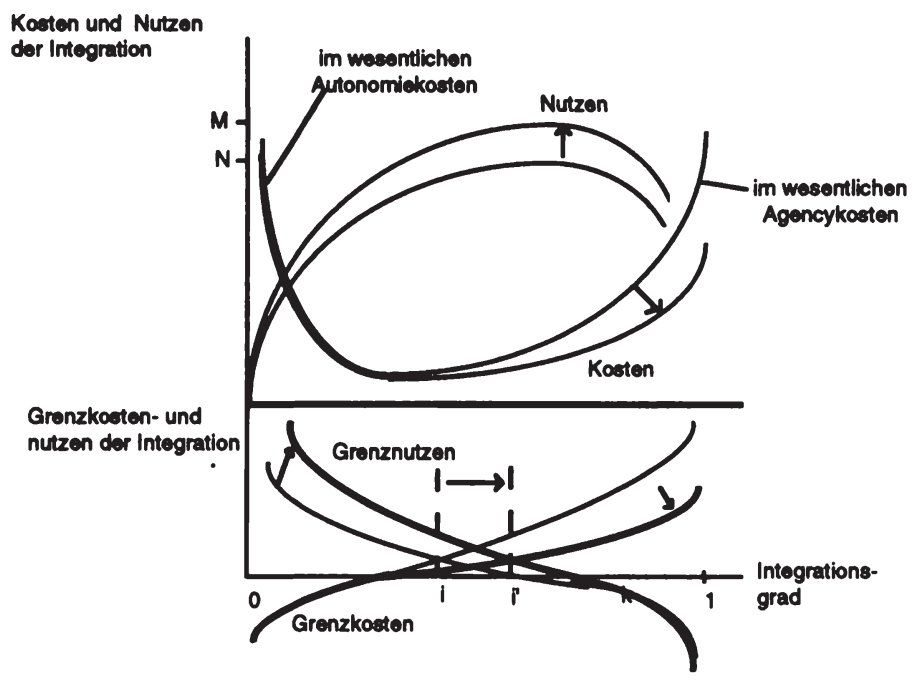

Abb. B.2.28: Steigender optimaler Integrationsgrad durch Einsatz von KIS

verändern. ${ }^{1}$ KIS senken vor allem die Administrationskosten und darüber den Verlauf der Agencykostenkurve. Demgegenüber verändern sich die quasi exogen gegebenen Autonomiekosten kaum. Auf den optimalen Integrationsgrad wirkt dies wie folgt: Durch den Einsatz von KIS möge sich das Nutzenpotential aus Integration von $\mathrm{N}$ auf $\mathrm{M}$ erhöhen. Somit verschiebt sich die Grenzintegrationskostenkurve "südöstlich" und die Grenzintegrationsnutzenkurve, zumindest links vom Nutzenmaximum,

1 Vgl. auch Venkatraman, N., Business Reconfiguration, a.a.O., S. 4. 
"nordöstlich". Folglich verschiebt sich der optimale Integrationsgrad i auf einen höheren Wert i'.1

Damit konnte allgemein der Zustand optimaler Integration definiert werden, welcher freilich im jeweiligen situativen Kontext operationalisierungsbedürftig ist. Es konnte allgemein die Wirkungsrichtung des Einsatzes von Informationstechnologie auf den optimalen Integrationsgrad skizziert werden.

Diese vorgestellte Sicht einer optimalen Integration hat aus Sicht der Fragestellung dieser Arbeit mehrere Vorteile gegenüber verwandten Ansätzen. Im Vergleich zum Integrationsmodell von Williamson beschränkt sie sich nicht nur auf den kontraktuellen Integrationsbegriff. ${ }^{2}$ Gegenüber dem Modell der optimalen Dezentralisierung von Jensen ${ }^{3}$ wurde vermieden, nur einen einzigen "locus of decision" anzugeben. Damit wird, anders als bei Jensen, das Integrationsproblem nicht nur durch die Zuordnung von Entscheidungsrechten an einem einzelnen Punkt angegangen. Auch im Vergleich zu Emery bzw. Frese wird das Integrationsproblem umfassender betrachtet und die Integrationskostenarten klarer unterschieden. ${ }^{4}$. Zudem wird versucht, nicht nur die Integrationskosten zu erfassen, sondern auch den Integrationsnutzen. Er ist aus dem Zusammenhang zum Zielsystem der Unternehmung zu identifizieren. Demgegenüber betrachten Jensen und auch Frese bzw. Emery lediglich die Agency Costs bzw. die Autonomiekosten als einzigen Vorteil eines höheren Integrationsgrades. Sie definieren diese Kosten im wesentlichen als Kosten der opportunistischen Subzielverfolgung. Jensen und Frese bzw. Emery sehen demzufolge den einzigen Nutzen einer engeren Koordination oder Integration im Sinken solcher Autonomiekosten durch bessere Überwachung und Kontrolle von

1 Zum gleichen Schluß kommt Emery, J.C., Management Information Systems, a.a.O., S. 255 ff.

2 Williamson, O.E., Economic Organization, a.a.O., S. 24; vgl. auch Abschnitt B.2.3.1.

3 Jensen, M.C., Meckling, W.H., Coordination, a.a.O., S. 9-21.

4 Frese, E., Organisation, a.a.O., S. 211. 
Mitarbeitern der Unternehmung. Integration wirkt durchaus auf Autonomie- und Agencykosten. Sie sind daher einbezogen worden. Der eigentliche Nutzen der Integration wird damit jedoch nicht einbezogen. ${ }^{1}$

\subsection{Zusammenfassende Darstellung des Bezugsrahmens}

Der im Teil B erarbeitete Bezugsrahmen ist in Abb. B. 29 zusammenfassend wiedergegeben. Er enthält die Strukturelemente der Integrationsentscheidung: die Zielgröße, die Definition des Integrationsbereichs, die situativen Bedingungen, die Integrationsinstrumente sowie ein Gerüst von Kosten und Nutzen der Integration.

Der Bezugsrahmen ist als entscheidungsorientierter situativer Ansatz für die Integrationsentscheidung zu verstehen. Im situativen Ansatz wird die interne Organisationsstruktur direkt, teilweise quasi-mechanistisch bzw. gesetzmäßig auf die Situation zurückgeführt, aus der verschiedenste Effizienzkritierien abgeleitet werden. ${ }^{2}$ Ein Beispiel ist die Studie von van de Ven, Delbecq und Koenig ${ }^{3}$, in der der Einsatz alternativer Koordinationsmechanismen aufgezeigt wird.

Kieser und Kubicek unterscheiden zwei Varianten des situativen Ansatzes, die analytische und die pragmatische bzw. handlungsorientierte. ${ }^{4}$ Die analytische Variante (z.B. Aston-Programm) gewinnt Erkenntnisse über die Auswirkungen eines (offen gelassenen) Situationsbegriffs auf die Organisationsstruktur durch statistische Analyse. Die pragmatische bzw. handlungs-

1 Eine Möglichkeit zur Einbeziehung des Nutzens von Integration in Transaktionskostenüberlegungen ist, den komparativen Nutzen alternativer institutioneller Arrangements als Opportunitätskosten der strategischen Position anzusehen. Vgl. Benkenstein, M., Henke, N., Vertikale Integration, a.a.O., S. 15

2 Vgl. hierzu und zum folgenden Picot, A., Ökonomische Theorien, a.a.O., S. 526.

3 Vgl. Van de Ven, A., Delbecq, A., Koenig, R.Jr., Coordination Modes, a.a.O.

4 Vgl. Kieser, A., Kubicek, H., Organisation, a.a.O., S. 57 ff., zum Wissenschaftsziel der Ansätze S. 58 f. 


\begin{tabular}{|c|c|c|c|c|c|}
\hline \multicolumn{3}{|c|}{ Crundmodell: Cebundener Wettbowerb } & \multicolumn{3}{|c|}{$\begin{array}{l}\text { Starre Faktoren } \\
\text { Entgangene Optionen } \\
\text { Organisationale Tragheit }\end{array}$} \\
\hline \multicolumn{6}{|l|}{$\begin{array}{l}\text { Zelgröea: } \\
\text { Dauer }\end{array}$} \\
\hline \multicolumn{3}{|c|}{ Integrationsbereich } & \multicolumn{3}{|c|}{ Sturtive Foldoren } \\
\hline \multicolumn{3}{|c|}{ 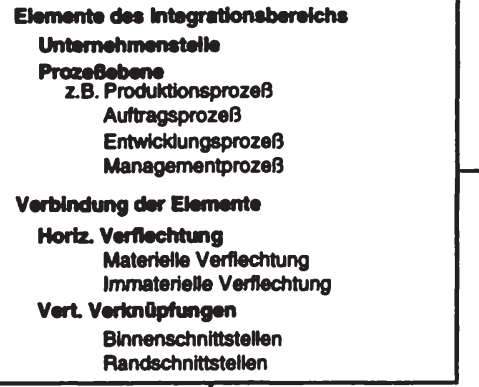 } & $\begin{array}{l}\text { Volumen } \\
\text { Unelcherh }\end{array}$ & $\begin{array}{l}\text { Menge } \\
\text { Frequen } \\
\text { Wlederh } \\
\text { Komplex } \\
\text { Verthalter } \\
\text { Naturtich } \\
\\
\text { Raumilct } \\
\text { Phystsch } \\
\text { Personal } \\
\text { Abnehm }\end{array}$ & $\begin{array}{l}\text { Iz } \\
\text { lolung } \\
\text { xitat } \\
\text { insunsicherhelt } \\
\text { he Unsicherhelt } \\
\text { h } \\
\text { h } \\
\text { ler-nieteranten }\end{array}$ \\
\hline \multicolumn{6}{|c|}{ Integrationsinstrumente: $3 \mathrm{~K}$} \\
\hline $\begin{array}{l}\text { Kommu } \\
\text { Informat }\end{array}$ & $\begin{array}{l}\text { intions- und } \\
\text { nesyetem }\end{array}$ & \multicolumn{2}{|c|}{ Koordinationsmechanismus } & & Kontralat \\
\hline \multirow{2}{*}{\multicolumn{2}{|c|}{$\begin{array}{c}\text { Abwicklungssysteme } \\
\text { Strukturiert Managment-Infor- } \\
\text { mationesysteme }\end{array}$}} & \multirow{2}{*}{\multicolumn{2}{|c|}{ Administrativ Plane }} & Markt & Eint.Tauschkontrakt \\
\hline & & & & \multirow{2}{*}{\multicolumn{2}{|c|}{\begin{tabular}{|ll} 
& Langtr. Rahmenkontr. \\
Relationale & Franchising \\
Kontrakte & Betelligungen \\
& Allanzen, Joint Vent. \\
& Interne Kontrahierung \\
\end{tabular}}} \\
\hline $\begin{array}{ll}\text { Semi- } & M \\
\text { strukturlert } & \text { sy } \\
& \text { DS } \\
\end{array}$ & $\begin{array}{l}\text { agement-Unterst.- } \\
\text { ame } \\
\text { ESS, GDSS }\end{array}$ & Strukturell & $\begin{array}{l}\text { hie } \\
\text { ntindungen }\end{array}$ & & \\
\hline \multicolumn{2}{|c|}{$\begin{array}{l}\text { Schwach- Expertensysteme } \\
\text { strukturiert Baroautomatisierung }\end{array}$} & \multicolumn{2}{|c|}{\begin{tabular}{|ll} 
Personal & $\begin{array}{l}\text { (In)Formale Teams } \\
\text { Ausblldung, Werte }\end{array}$ \\
\end{tabular}} & $\begin{array}{l}\text { Unter- } \\
\text { nehmung }\end{array}$ & $\begin{array}{l}\text { Diensthontrakt } \\
\text { Gesellechaftskontrakt }\end{array}$ \\
\hline \multicolumn{3}{|c|}{ Kosten der Integration (") } & \multicolumn{3}{|c|}{ Nutzen der integration } \\
\hline $\begin{array}{l}\text { Autonomie- } \\
\text { kosten } \\
\text { Agency- } \\
\text { kosten }\end{array}$ & \multicolumn{2}{|c|}{$\begin{array}{l}\text { Wagnisse für Subziele von Agenten } \\
\text { Abschoptung von Quasi-Rente } \\
\text { Nachlassigkeitskosten } \\
\text { Informationskosten } \\
\text { Anreizkosten } \\
\text { Kontrolikosten }\end{array}$} & \multicolumn{3}{|l|}{$\begin{array}{l}\text { Zeit } \\
\text { Qualităt } \\
\text { Kosten } \\
\text { Stabilităt } \\
\text { Verteilung } \\
\text { Flexibilităt }\end{array}$} \\
\hline
\end{tabular}

(") Etwaige durch Integration begründete Qualitatseinbußen, Flexibilltătsverluste oder Zeltnachtelle werden zur Vermeidung der Doppelautzahlung als negativer Nutzen eingeordnet.

Abb. B.229: Bexugarehmen der integrationsentecheldung 
orientierte Variante geht von einem rationalen Entscheider aus. Vereinfacht geht es um die Auswahl von Aktionsparametern unter bestimmten situativen Bedingungen zur Erfüllung von Zielen.

Mit dem Bezugsrahmen der Integrationsentscheidung werden Voraussetzungen zur Analyse der Integrationsentscheidung geschaffen. Er stellt die Variablen der Integrationsentscheidung dar, operationalisiert diese und stellt die Bezüge zwischen den Variablen her: Dabei ist ein konkreter Integrationsbereich zu definieren, es sind die situativen Bedingungen zu analysieren um die Integrationsinstrumente KIS, Koordinationsmechanismen und Kontrakte in Abhängigkeit von ihren Kosten- (Agencykosten, Autonomiekosten) und Nutzenwirkungen (Wettbewerbsposition, Dauerhaftigkeit) auszuwählen. Dies entspricht methodologisch dem entscheidungsorientierten situativen Ansatz der Organisationstheorie.

Demgegenüber liegt bei der ergänzend verwendeten Herleitung des optimalen Integrationsgrades methodologisch die ökonomische (Organisations-) Theorie zugrunde, in die Vorteilhaftigkeit alternativer institutioneller Arrangements anhand der komparativen Zielwirkungen bewertet wird.1 Beide Sichtweisen tragen zum Erklärungsziel dieser Arbeit bei. Der ökonomische Ansatz ergab ein Verständnis des Optimalitätszustandes sowie der Kosten- und Nutzenkategorien, der organisationstheoretische eine Verbindung von Situation und Integrationsentscheidung.

1 Vgl. Williamson, O.E., Economic Organization, a.a.O., S. 17 


\section{Wettbewerbsstrategische Wirkung von Integration - Explorative Untersuchung unter besonderer Beriicksichtigung von KIS}

Auf Basis des im Teil B erarbeiteten Bezugsrahmens kann die wettbewerbsstrategische Wirkung von Integration analysiert werden. Wie aus dem Untertitel dieser Arbeit hervorgeht, erfolgt dies unter besonderer Berücksichtigung der KIS. Dies erscheint sinnvoll aufgrund der eingangs aufgezeigten hohen Bedeutung der KIS für die Integration von Geschäftsaktivitäten einerseits, anderseits aufgrund der weitreichenden Substitution der Koordinationsmechanismen und Kontrakte durch KIS.

\section{Reichweiten der Integration und Integrationsstrategien}

Die Kosten- und Nutzenwirkungen von Integration lassen sich, wie dargelegt, nur im Kontext der spezifischen Bedingungen eines Integrationsbereichs abschätzen. Deswegen wird die wettbewerbsstrategische Wirkung von Integration anhand einer explorativen Untersuchung von Fallstudien analysiert. Um allerdings hieraus generalisierbare Erkenntnisse über Kosten- und Nutzenwirkungen von Integration ableiten zu können, wurden Gruppen von Fallstudien gebildet, die jeweils vergleichbare Integrationsstrategien aufweisen. Dies soll eine hinreichend generalisierbare, aber auch hinreichend kontextspezifische Diskussion der Wirkungen der Integration auf den dauerhaften Wettbewerbsvorteil ermöglichen. Eine erste Strukturierung verschiedener Integrationsstrategien ist auf Basis der fünf Ebenen der "IT-induzierten Rekonfiguration von Unternehmungen" von Venkatraman möglich ${ }^{1}$ (vgl. Abb. C.1.1).

Jede Ebene bezeichnet dabei eine unterschiedliche Reichweite von Integration mit KIS, und zwar ausgehend von lokalem Einsatz (keine Integration), über interne Integration verschiedener Geschäftsaktivitäten, Neugestaltung des Geschäftsprozesses, Neugestaltung der Beziehungen zwischen den Geschäftsaktivitäten über die Unternehmensgrenzen hinweg bis zur Schaffung völlig neuer Geschäftsfelder durch KIS. Diesen Ebenen werden allgemeine, mit der Ebene zunehmende Nutzenbandbreiten zugeschrieben. Von den unteren "evolutionären" Ebenen werden die "revolutionären" Ebenen unterschieden. Bei diesen steht nicht die Unterstützung der bisherigen

1 Vgl. Venkatraman, Business Reconfiguration, a.a.O., S. 5 - 6. 
Konfiguration von Geschäftsaktivitäten im Vordergrund, sondern eine Rearchitektur der Unternehmung.

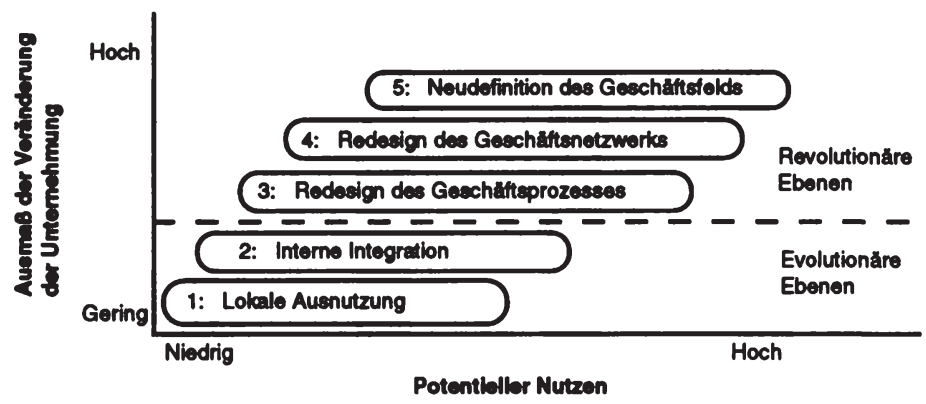

Abb. C.1.1 Fün Ebenen der IT-induzierten Rekonfiguration von Unternehmungen

Quelle: Venkatraman, N. IT-induced Business Reconfiguration, Author's Draft. MIT 1989, S. 6

Mit dem Ansatz von Venkatraman ist zwar eine grobe Strukturierung von Ebenen und "Nutzenwirkungen" möglich, jedoch ist der Ansatz nicht hinreichend, um einzelne Integrationsstrategien $\mathrm{zu}$ unterscheiden und deren Wirkung auf den dauerhaften Wettbewerbsvorteil der Unternehmung zu analysieren. Allerdings ist eine weiter differenzierende Unterscheidung verschiedener Reichweiten auf Basis der drei mittleren Ebenen möglich.1 Diese werden im folgenden als interne Integration, Geschäftsprozeßintegration und Geschäftsnetzwerkintegration bezeichnet.

Diese Reichweiten werden nach der Anzahl der beteiligten Unternehmen und nach dem Anteil des Integrationsbereichs am Geschäftsprozeß weiter in sechs Integrationsstrategien unterschieden: innerhalb der internen Integration die Transaktionsintegration und die Organisationsintegration, innerhalb der Geschäftsnetzwerkintegration die Information Partnerships, die vertikalen Netzwerke sowie die elektronischen Märkte, daneben die Geschäftsprozeßintegration

(vgl. Abb. C.1.2).

1 Demgegenüber beziehen sich die erste Ebene auf Automatisierungseffekte durch KISEinsatz (lokale Ausnutzung), die fünfte Ebene auf neue Märkte durch KIS-Einsatz. 


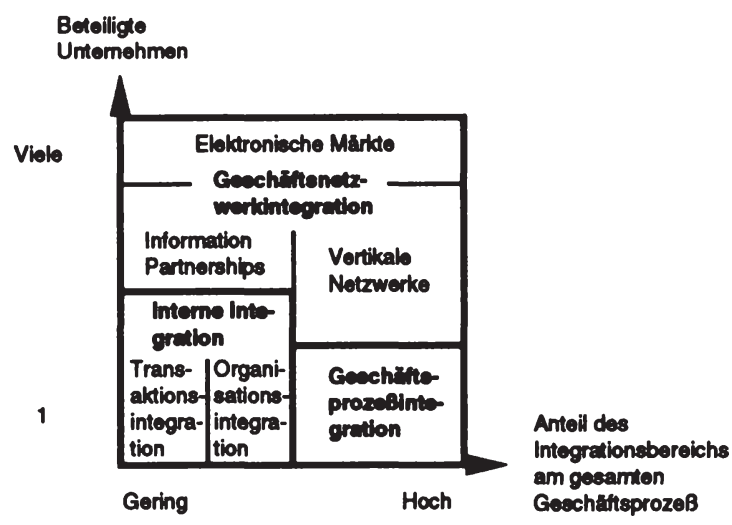

Abb. C.1.2: Systematisierung von Integrationsotrategien bei Einsatz von KIS

\subsection{Interne Integration}

Interne Integration zielt bei gegebener Struktur der Geschäftsaktivitäten und Unternehmensteile auf die Schaffung integrativer Verbindungen innerhalb der Unternehmung. Nach der Art der integrativen Verbindungen können zwei Integrationsstrategien unterschieden werden: die auf vertikale Verknüpfungen gerichtete Transaktionsintegration und die auf horizontale Verflechtungen gerichtete Organisationsintegration (vgl. Abb. C.1.2).

Die Transaktionsintegration zielt darauf, durch KIS-Unterstützung vertikaler Verknüpfungen nacheinandergelagerte Geschäftsaktivitäten wesentlich enger zu verzahnen. Der Integrationsbereich umfaßt Produktions- und Auftragsprozesse und wird von hohem Volumen und geringer Unsicherheit geprägt sein. Als Integrationsinstrumente kommen damit in Frage Abwicklungssysteme, administrative Koordinationsmechanismen und aufgrund der internen Orientierung firmenkonstituierende Kontrakte.

Während die Transaktionsintegration vor allem auf die vertikale Binnenverknüpfung ausgerichtet ist, wird mit der Organisationsintegration angestrebt, immaterielle horizontale Verflechtungen innerhalb der Unternehmung zu schaffen. Sinn der horizontalen Verflechtung ist die Verbindung gleichartiger Geschäftsaktivitäten bzw. bei Einsatz von KIS gleichartiger Informationen. Dabei wirkt die Organisationsintegration zu- 
meist indirekt über die Verbesserung von Entscheidungsprozessen und Kommunikationsmöglichkeiten. Die Organisationsintegration ist damit, anders als die Transaktionsintegration, zumeist auf den Entwicklungs- und Managementprozeß gerichtet, der durch geringere Volumina und schwächeren Strukturierungsgrad gekennzeichnet ist. Entsprechend werden andere Instrumente zur Organisationsintegration eingesetzt, nämlich Management-Unterstützungssysteme, personelle und strukturelle Koordinationsmechanismen sowie aufgrund hoher Spezifität firmenkonstituierende Kontrakte.

\subsection{Geschäftsprozeßintegration}

Die Geschäftsprozeßintegration betrifft den gesamten Geschäftsprozeß einer Unternehmung. Diese richtet ihr Geschäft sowie die beiden anderen Integrationsinstrumente fundamental und umfassend auf die KIS aus. Daher wird eine solche Unternehmung auch als KIS-basierte Unternehmung bezeichnet. Schwerpunkt der Geschäftsprozeßintegration ist die Bildung elektronischer vertikaler Verknüpfungen. Durch konsequente Abstimmung der Geschäftsaktivitäten mit dem jeweiligen Strukturierungserfordernis von Abwicklungs-, Informations- und gelegentlich EntscheidungsUnterstützungssystemen werden vertikale Verknüpfungen zur Abwicklung hochvolumiger Geschäftsprozesse geschaffen. Dies wird bei nur begrenzter (Umwelt-) Unsicherheit der Fall sein.

\subsection{Geschäftsnetzwerkintegration}

Mit der Geschäftsnetzwerkintegration wird die Architektur des Geschäftsprozesses über die kontraktuellen Unternehmensgrenzen hinaus verändert. ${ }^{1}$ Die Geschäftsnetzwerkintegration zielt damit auf die Überwindung von Schnittstellen und die Nutzung von Gleichartigkeiten zwischen verschiedenen Unternehmungen, kann also sowohl vertikale Verknüpfungen als auch horizontale Verflechtungen umfassen. Der wichtigste Unterschied zu den vorstehenden Integrationsformen ergibt sich aus dem integrativen Zusammenwirken von Unternehmensteilen, die unterschiedlichen Zielfunktionen (Unternehmungen) unterstehen. Dies wird nur durch die Be-

1 Vgl. auch Venkatraman, N., Business Reconfiguration, a.a.O., S. 5 - 6. 
grenzung der Spezifität von Faktoren möglich sein, weswegen sich die Geschäftsnetzwerkintegration durch KIS überwiegend auf den Auftrags- und Produktionsproze $B$, weniger auf den (oft firmenspezifischen) Managementund Entwicklungsproze $B$ konzentrieren wird. Dabei können die Information Partnerships, die vertikalen Netzwerke und die elektronischen Märkte unterschieden werden.

Vertikale Netzwerke sind elektronische vertikale Verknüpfungen über verschiedene Stufen eines Geschäftssystems, die von einer Unternehmung (focal Firm) getragen werden. Bekannte Beispiele vertikaler Netzwerke sind die Reservierungssysteme der Fluggesellschaften oder die Bestellsysteme von Pharmagroßhändlern. Die focal Firm im vertikalen Netzwerk trägt ein KIS. Sie hat vor- und/oder rückwärtsgerichtete elektronische Verbindungen in das Geschäftsnetzwerk. Damit betreffen sie vorwiegend den Auftragsprozeß, weniger den Managementprozeß. Entsprechend stehen bei den Instrumenten Abwicklungssysteme, administrative Koordinationsmechanismen und relationale Kontrakte im Vordergrund. Charakteristisch für vertikale Netzwerke sind hohe Datenvolumina und nur mäßige Unsicherheit. Eine Bildung elektronischer Verknüpfungen wird oft kunden- bzw. lieferanten-, räumlich-physisch- und personalspezifisch sein.

Information Partnerships sind Gruppen von unabhängigen Unternehmen, die durch KIS elektronische, überwiegend immaterielle, horizontale Verflechtungen bilden. Die Information Partnerships richten sich damit nicht in erster Linie auf die Abwicklung des Geschäftsprozesses der beteiligten Unternehmen, sondern auf den Informationsaustausch zwischen gleichartigen Geschäftsaktivitäten, z.B. der gemeinsamen Nutzung oder gar dem Aufbau von Datenbanken.1

Elektronische Märkte können als vertikale Netzwerke angesehen werden, die von einem Marktträger (Market Maker) getragen werden, der selbst nicht Teil des eigentlichen Geschäftsprozesses ist. Die Charakterisierung als Markt weist bereits darauf hin, daß die Beziehungen der Marktteilnehmer nicht durch kontraktuelle Integration, sondern durch Marktkontrakte und langfristigere, marktorientierte relationale Kontrakte geregelt werden.

1 Vgl. Malone, T., Yates, J, Benjamin, R.I., Electronic Markets, a.a.O., S. 495 - 496. 


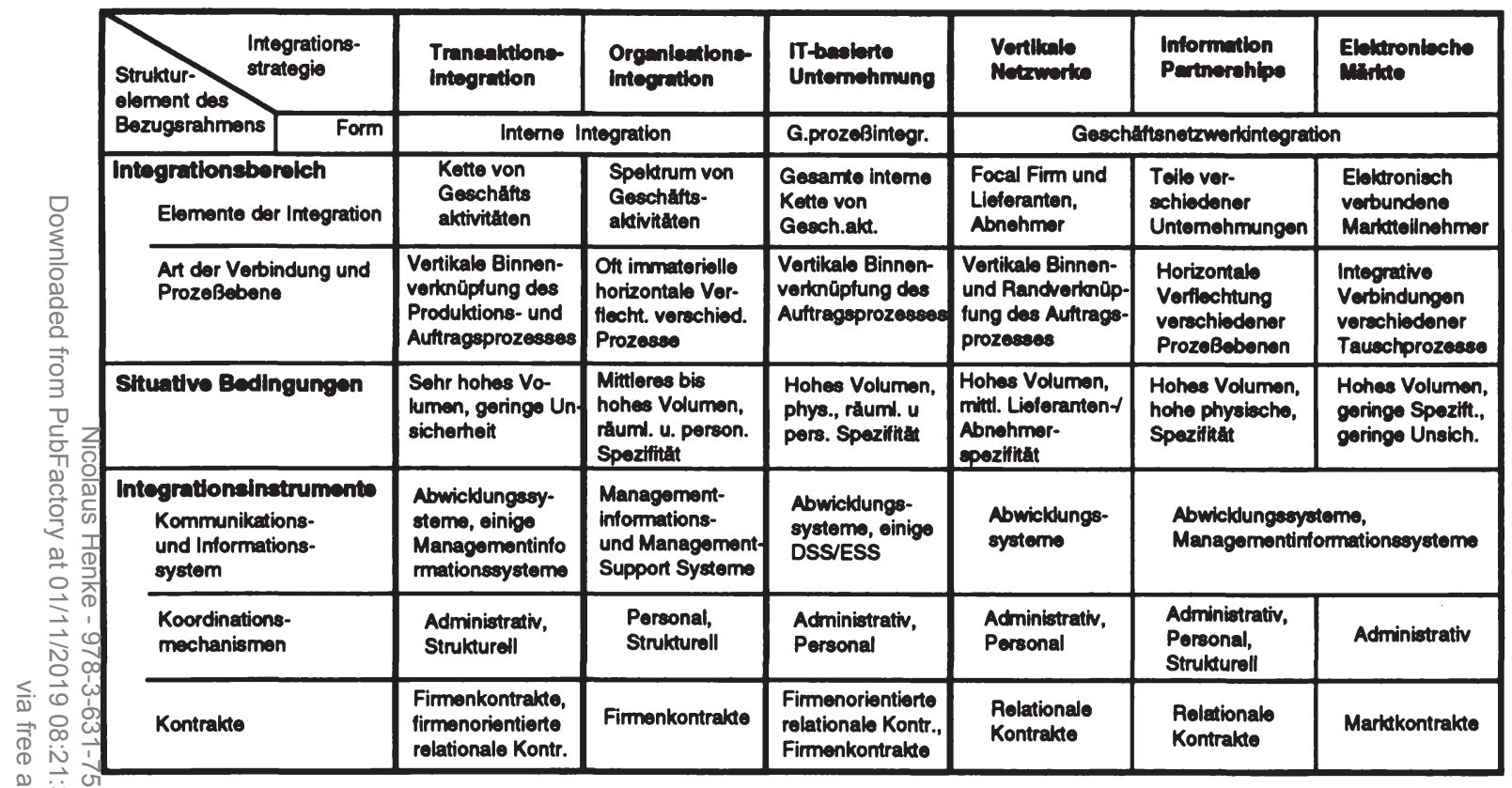

Abb. C.1.3: Unterscheidung der Integrationsstrategien anhand der Strukturelemente des Bezugsrahmens 
Basis der explorativen Untersuchung bilden Berichte aus 35 verschiedenen Unternehmungen, überwiegend aus den USA, die ein oder mehrere KIS zur Bildung integrativer Verbindungen einsetzen. Die Fallstudien wurden durch breit angelegte Recherchen mittels Datenbanken, Katalogen, Literatursammlungen verschiedener Art und Experteninterviews identifiziert. ${ }^{1}$ Bei der Selektion der Fallstudien wurde zwar die Grundgesamtheit mit großer Sorgfalt bestimmt, die bekannten (d.h. veröffentlichten) Fälle werden jedoch nicht annähernd vollständig erfaßt worden sein. Ein Indiz dafür, daß dabei zumindest offensichtliche Lücken vermieden werden konnten, ist die Tatsache, daß der von den beiden wesentlichen Dimensionen 'beteiligte Unternehmen' und 'Anteil des Integrationsbereichs' aufgespannte Raum (vgl. Abb. C.1.2.) abgedeckt wird. Bei Beschränkung der Untersuchung auf eine Branche oder eine anderweitig abgrenzbare Gruppe von Unternehmungen wäre eine vollständige Abdeckung zwar möglich gewesen, die Vielfalt der Probleme und Möglichkeiten einer Integrationsstrategie, die sich bei ganz unterschiedlichen Integrationsbereichen, situativen Bedingungen und Integrationsinstrumenten ergeben, hätte jedoch nicht analysiert werden können. Somit hätte auch das Ziel der explorativen Untersuchung nicht erreicht werden können.

Ziel war es nicht, die Zahl der Fallstudien zu maximieren. ${ }^{2}$ Vielmehr soll auf Grundlage des im Teil B erarbeiteten Bezugsrahmens - an Beispielen aus der Praxis aufgezeigt werden, welche Faktoren eine Integrationsstrategie gelingen oder scheitern lassen. Mit anderen Worten, welche Faktoren in welchem Maße zur Erlangung von Positionsvorteilen und zu ihrer dauerhaften Absicherung beitragen. Auf Basis der aus einzelnen Fallstudien gewonnenen Erkenntnisse werden dann Zusammenhänge extrahiert und daraus die strategische Wirkung der einzelnen Integrationsstrategien geschlossen. Selbstverständlich besteht dabei ein Zielkonflikt zwischen dem Abstraktions- und Aggregationsniveau einerseits und der Verifizierbarkeit oder Falsifizierbarkeit der Gesamtaussage andererseits.

1 Quellen sind Zeitschriftenveröffentlichungen, Buchveröffentlichungen, Lehrfallstudien, Datenbankauskünfte, Arbeitspapiere, Forschungsberichte, Geschäftsberichte, Mitschriften von Vorträgen, Interviews mit Entscheidungsträgern und direkte Recherchen.

2 Ein Vielfaches liegt in der Literatur vor. Vgl. eine deutsche Analyse von Mertens, P., Schumann, M., Hohe, U., Informationstechnik als Mittel zur Verbesserung der Wettbewerbsposition - Erkenntnisse aus einer Beispielsammlung, in: Informationstechnologie und strategische Führung, (Hrsg.), Spremann, K., Zur, E., Wiesbaden, 1989, S. 109 - 136. 
Das Ziel der explorativen Untersuchung, nämlich festzustellen, welche Faktoren in welchem Maße zur Erlangung von Positionsvorteilen und zu ihrer dauerhaften Absicherung beitragen, führt zu einer gewissen Prädetermination des Untersuchungsverfahrens. Dies ist zum einen die Verzerrung der Grundgesamtheit durch ein vermutetes Übergewicht "erfolgreicher" Integrationsanwendungen. Denn bei der Auswahl der Fallstudien wurden diejenigen bevorzugt, die eine hinreichende Informationsbasis zur Diskussion der Strukturelemente des Bezugsrahmens bieten. Beispiele für Mißerfolge sind zudem tendenziell schwerer einbeziehbar, da die Implementierung häufig abgebrochen wird oder Informationen darüber nicht veröffentlicht werden. Es erscheint jedoch unmöglich, die dadurch entstehende Verzerrung systematisch zu beheben. Möglich ist nur, auch bewußt weniger erfolgreiche Anwendungen zu identifizieren und einzubeziehen.

Bei der Auswertung der Fallstudien wird auf eine "quantitative Objektivierung" verzichtet. Durch Umsetzung der einzelnen Teildimensionen in kardinal oder ordinal skalierbare Werte und entsprechende Bewertung aller Fallstudien anhand dieser Skalen wäre zwar eine "quantitative Objektivierung", etwa im Hinblick auf genauere Korrelationen zwischen Erfolgs- und Strukturdimensionen möglich. Hiervon wurde jedoch aus mehreren Gründen abgesehen. Zunächst müßte eine Messung von Erfolgsgrößen an Umsatz- oder Rentabilitätsmaßen ansetzen. Für das Problem der strategischen Integration ist jedoch das langfristige Erfolgspotential der Unternehmung als Referenzparameter zu wählen, untergliedert in Wettbewerbsposition und Dauerhaftigkeit. ${ }^{1}$ Außerdem böten sehr wenige Fallstudien eine hinreichende Informationsbasis, um sämtliche Teilgrößen von Kosten und Nutzen sinnvoll zu operationalisieren. Dies ist auch nicht der Zweck der Unterscheidung in Teilgrößen. Vielmehr wird die konzeptionell-analytische Vollständigkeit eines (präskriptiven) Kosten-/Nutzen-Gerüsts angestrebt, nicht dessen empirisch detaillierter Beleg. Darüber hinaus ist es angesichts der qualitativ-inhaltlichen Selektion der Fallstudien fraglich, ob und wie stark die Validität durch eine Umsetzung in kardinal oder ordinal skalierbare Informationen tatsächlich gesteigert wird.

1 Vgl. Abschnitt B.1.3 sowie Gälweiler, A., Unternehmensplanung, Grundlagen und Praxis, Frankfurt, New York, 1974, S. 230. Zu den Beziehungen zwischen Erfolgspotential und (Perioden-)Erfolg vgl. Gälweiler, A., Unternehmenssicherung und strategische Planung, in: Zeitschrift für betriebswirtschaftliche Forschung, 28. Jg., 1976, S. 363 - 376. 
Diese Schwierigkeiten sind auch aus der Literatur zum strategischen Einsatz von Informationstechnologie ersichtlich, in der drei verschiedene Forschungsstrategien eingeschlagen werden.1 Die erste Richtung, die konzeptionell-theoretische Diskussion, welche Bedeutung der Einsatz von Informationstechnologie für die Unternehmensstrategie hat, ${ }^{2}$ war auch das Ziel dieser Arbeit. Die zweite Richtung ist die empirische Analyse der Auswirkungen des KIS-Einsatzes; analysiert werden dabei entweder einzelne Parameter bzw. Teildimensionen ${ }^{3}$ oder Auswirkungen auf relativ aggregiertem Niveau. 4 Die dritte Richtung sind detaillierte Untersuchungen von Fallstudien, die die Wirkung des KIS-Einsatzes in einem konkreten situativen Kontext ermitteln, einschließlich der Entwicklung im Zeitablauf. ${ }^{5}$ Den verschiedenen Richtungen liegt ein Zielkonflikt zwischen der Generalität getroffener Aussagen und der möglichen Tiefe der empirischen Analyse zugrunde.

Diesem Zielkonflikt soll dadurch begegnet werden, daß im folgenden der entwickelte Bezugsrahmen, einzelne Fallstudien und breiter definierte Intregrationsstrategien kombiniert diskutiert werden. Dazu wird jeweils wie folgt vorgegangen. Im ersten Schritt wird eine für die betrachtete Reichweite typische Kurzfallstudie ausführlich diskutiert. Diese untergliedert

1 Vgl. Venkatraman, N., Short, J.E., Strategies for Electronic Integration: From Order-Entry to Value-Added Partnerships at Baxter, Author's Draft, Massachusetts Institute of Technology, Cambridge, Massachusetts, 1990, S. 3.

2 Vgl. z.B. Parsons, G.L., Information Technology, a.a.O., S. 3 - 13; Porter, M.E., Millar, V.E., Information, a.a.O., S. 149 - 160; McFarlan, F.W., Information Technology Changes the Way You Compete, Harvard Business Review, Vol. 62, May-June 1984, S. 98 - 103; Barrett, S., Konsynski, B., Inter-Organization Information Sharing Systems, in: MIS Quarterly, Vol. 8, 1982, S. 93 - 105; Johnston, H.R., Vitale, M.R., Creating Competitive Advantage with Interorganisational Information Systems, in: MIS Quarterly, June, Vol. 12, 1988, S. 153 - 165; Wiseman, Ch., Attack \& Counterattack: The New Game in Information Technology, in: Planning Review, September/October 1988, S. 6 - 12; Malone, T., Yates, J, Benjamin, R.I., Electronic Markets, a.a.O., S. 484 - 497; Bakos, Y.J., Interorganizational Information Systems: Strategic Implications for Competition and Coordination, Unpublished Doctoral Dissertation, Massachusetts Institute of Technology, Cambridge, Massachusetts, 1987; Meyersiek, D., Jung M., Kopplung von System- und Unternehmensstrategie als Voraussetzung für Wettbewerbsvorteile, in: Informationstechnologie und strategische Führung, (Hrsg.), Spremann, K., Zur, E., a.a.O.; Nagel, K., Bewertung strategischer Vorteile, a.a.O., S. 49 - 64.

3 Vgl. z.B. Venkatraman, N., Zaheer, A., Electronic Integration and Strategic Advantage: A Quasi-Experimental Study in the Insurance Industry, International Conference on Information Systems, Boston, Massachusetts, December 4 -6, 1989.

4 Vgl. z.B. Mertens, P., Schumann, M. Hohe, U., Informationstechnik, a.a.O.

5 Vgl. z.B. Copeland, C.G., McKenney, J.L. Airline Reservations Systems: Lessons from History, in: MIS Quarterly, Vol. 12, September 1988, S. 353 - 370; Clemons, E.K., Row, M., McKesson Drug Company: A Case Study of Economost - A Strategic Information System, in: Journal of Management Information Systems, Vol. 5, 1988, S. 36 - 50. 
sich in (1) die Beschreibung des Integrationsbereichs anhand der Strukturelemente des Bezugsrahmens und (2) die Analyse der Auswirkungen der Integration auf die Wettbewerbsposition und deren Dauerhaftigkeit. Im zweiten Schritt werden jeweils mehrere Fallstudien zu einer Integrationsstrategie vergleichend analysiert.

\subsection{Interne Integration}

Bei der internen Integration wurde die Transaktionsintegration und die Organisationsintegration unterschieden. Die Transaktionsintegration zielt auf die Abwicklung des Auftragsprozesses meist über vertikale Verknüpfungen. Die Organisationsintegration ist dagegen auf die horizontale Verflechtung zumeist des Managementprozesses gerichtet. Diese Integrationsstrategien werden im folgenden an verschiedenen Fallstudien diskutiert. Die eingangs herangezogene Kurzfallstudie der Frito Lay, Inc. erscheint zur Illustration der internen Integration besonders geeignet, weil Frito Lay nicht nur beide Integrationsstrategien der internen Integration einsetzt, sondern diese auch kombiniert.

\subsubsection{Interne Integration bei Frito Lay, Inc.}

Frito Lay, Inc. erzielte ${ }^{1}$ mit der Produktion und dem Verkauf salziger Snacks innerhalb der USA einen Umsatz von $\$ 4,2$ Mrd. bei einem Rohgewinn (Operating Profit) von deutlich über $\$ 700$ Mio. (1990). Damit war Frito Lay Marktführer in vielen Teilsegmenten des $\$ 20 \mathrm{Mrd}$. umfassenden Snack Food Marktes; die Marktanteile lagen zwischen $28 \%$ im \$2,5 Mrd. großen Segment für Kartoffelchips und $90 \%$ im $\$ 1$ Mrd. großen Segment für Maischips. Frito Lay strebte ein jährliches reales Umsatzwachstum von $6 \%$ und jährlich ein zweistelliges Gewinnwachstum an. ${ }^{2}$ Frito Lay war die bedeutendste Division von PepsiCo, Inc.

1 Im Gegensatz an theoretischen Aussagen sind Fallstudien, der internationalen Praxis folgend, im Imperfekt geschrieben. Damit wird der situative Charakter am Erhebungszeitpunkt wiedergegeben und verdeutlicht, daB die getroffenen Aussagen im Zeitablauf mit der Veränderung der Situation anzupassen sind.

2 Das Marktvolumen wuchs jährlich real um $3 \%$. Die Marktanteilszahlen stammen aus dem Jahr 1986. Vgl. Linder, J., Mead, M., A Strategic Transition (A), Boston, Massachusetts, Harvard Business School Case No. 9-187-065, 1986, S. 2. 


\subsubsection{Der Integrationsbereich: Auftragsprozeß und operative Steuerung}

Frito Lay verzeichnete in den siebziger Jahren ein hohes Umsatzwachstum mit den Produktionskapazitäten als wichtigstem Engpaß. In den frühen achtziger Jahren stockte das Wachstum. Zunehmend wurde der Absatz zum Engpaß. Dies lag insbesondere an den vorhandenen administrativen Koordinationsinstrumenten zur Abwicklung des erheblichen Volumens von Transaktionen. Ein schematisches Mapping des Auftragsprozesses von der Produktion bis zum Abverkauf, einschlieBlich der operativen Steuerung, ist in Abb. C.2.1 dargestellt.

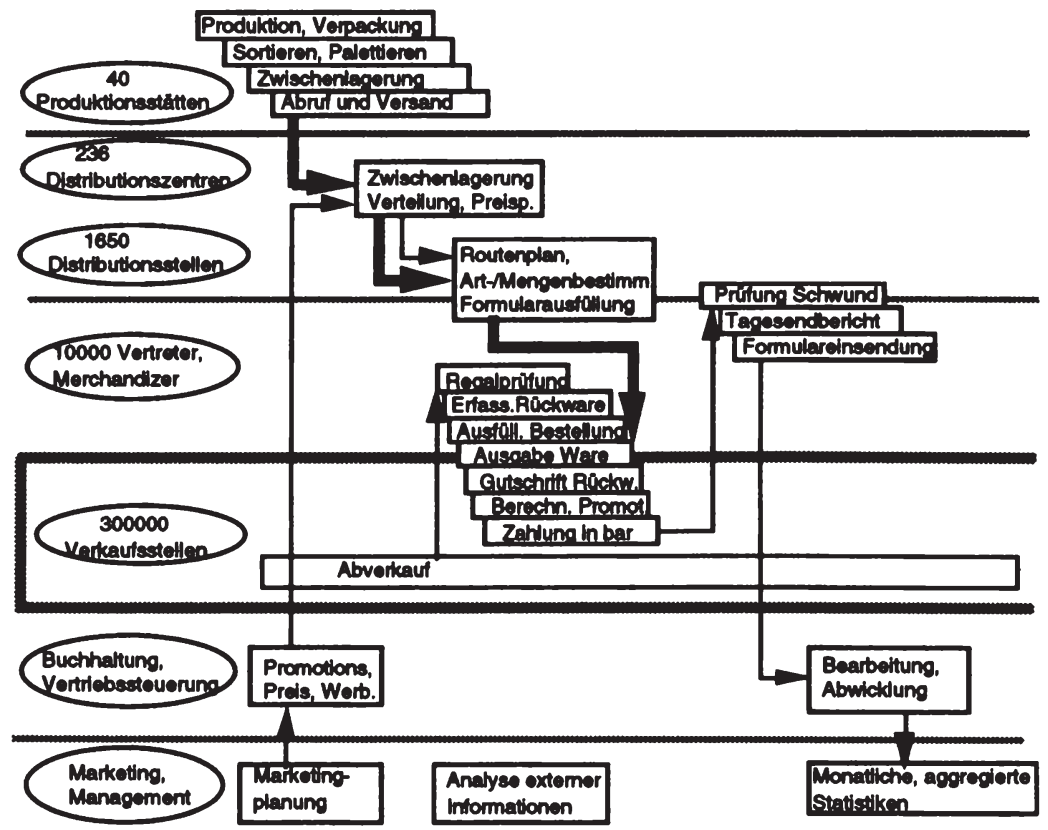

Abb. C.2.1: Fitto Lay, Inc., Map, Integrationsbereich Auttragsabwicklung und operative Steuerung

Charakteristisch für die situativen Bedingungen im Integrationsbereich waren ein extrem hohes Volumen, mittlere Umweltunsicherheit und rela- 
tiv hohe Spezifität (des Distributionsnetzes). Die Spezifität ergab sich aus der Ausrichtung eines USA- weiten Distributionsnetzes auf die physischen, räumlichen Bedingungen sowie der Abnehmer von Frito Lay. Umweltunsicherheit bestand insbesondere in Regionalmärkten, wo Frito Lay sowohl mit einigen USA-weit anbietenden Markenartiklern, als auch mit Anbietern regionaler Spezialitäten konkurrierte. Diese boten nicht nur kleinere, regionale Marken (Absatzvolumen $\$ 20$ Mio.) an, sondern variierten auch neben der Produktpolitik andere Marketinginstrumente wesentlich flexibler, als dies Frito Lay möglich war.

Der Integrationsbereich war durch ein extrem hohes Volumen von $\mathrm{Ge}-$ schäftsaktivitäten gekennzeichnet. Wöchentlich wurden 500.000 Lieferungen an 300.000 Verkaufsstellen von 10.000 Vertretern bzw. Merchandizern vorgenommen, die von 40 Produktionsstätten über 236 Distributionszentren und 1650 Distributionsstellen verteilt wurden. Jede einzelne Lieferung enthielt dabei eine Fülle von Produkten. Noch 1985 hatte Frito Lay 100 Marken angeboten, 1990 wurden, um den regionalen Wettbewerbern zu begegnen, $\mathbf{4 0 0}$ Marken geführt, wovon die meisten in mehr als zwei verschiedenen Verpackungsgrößen verkauft wurden. ${ }^{1}$

Abb. C.2.1 zeigt schematisch den Integrationsbereich der Auftragsabwicklung bei Frito Lay mit den wesentlichen Geschäftsaktivitäten und den sie tragenden Unternehmensteilen. ${ }^{2}$ Abgebildet wird dabei ein einzelner Auftragszyklus. Hintereinandergelagerte, aber unmittelbar zusammenhängende Geschäftsaktivitäten sind dabei als verschobene Blockgruppen zusammengefaßt worden, wie etwa die Geschäftsaktivitäten des Produktionsbereichs. Breite (einfache) Pfeile stehen für Materialflüsse (Informationsflüsse) außerhalb der Blockgruppen. Nach der Produktion wurde die Ware täglich in die Distributionsstellen verteilt. Die dort tätigen Außendienstmitarbeiter erfaßten und lagerten die Ware, nahmen gleichzeitig damit auch Preis- und Promotion-Regelungen entgegen und verteilten schließlich die Ware an die einzelnen Verkaufsstellen weiter. Im Diagramm wird das Verstreichen der Zeit durch eine Rechtsverschiebung ge-

1 Vgl. Linder, J., Mead, M., A Strategic Transition (A), a.a.O., S. 4 - 6, Crutchfield, R.J., Applications: Getting a Leg Up by Using Handhelds, in: Datamation, Vol. 33, No. 1, 1987, S. 32; Beck, L., Frito Lay Takes a Fresh Approach to Automation, in: Modem Materials Handling, Vol. 42, No. 1, 1987, S. 79.

2 Vgl. Linder, J., Mead, M., A Strategic Transition (A), a.a.O., S. 5 - 19; Crutchfield, R.J., Applications, a.a.O., S. 32; Beck, L., Frito Lay, a.a.O., S. 79; Main, T., Computers of the World, Unite!, in: Fortune, Vol. 122, No. 7, 1990, S. 113 - 122. 
kennzeichnet, allerdings nur in ungefährer, nicht maßstabsgenauer Darstellung. 1

Die bildliche Darstellung der Abwicklung von monatlich 2 Millionen Transaktionen mit bis zu 400 verschiedenen Artikeln pro Transaktion vermittelt einen Eindruck der Dimension des hier vorliegenden Integrationsproblems. Dabei treten zwei schwer überwindbare Schnittstellen hervor. Dies ist einerseits die unmittelbare Abwicklung zahlreicher Folgeaktivitäten, bei der viele Doppelarbeiten anfallen, und andererseits die Entfernung des Marketing/Managements vom eigentlichen Geschäft, auch von den Informationsgrundlagen.

\subsubsection{Einsatz von KIS im Integrationsbereich}

Mit zwei großen Projekten hat Frito Lay die interne Integration der Geschäftsaktivitäten neu ausgerichtet: die Einführung von Hand-Held-Computern für die Außendienstmitarbeiter sowie die Einführung eines umfangreichen unternehmensweiten Management-Unterstützungssystems. Dabei entspricht die Grundlogik des Hand-Held-Computer-Projekts der internen Transaktionsintegration und die des Management Support Systems der internen Organisationsintegration (vgl. Abb. C.1.2).

In der gesamten Abwicklung der Distribution wurden zahlreiche administrative Koordinationsmechanismen durch den Einsatz der Hand-HeldComputer substituiert oder erheblich verändert. Jeder Außendienstmitarbeiter erhielt einen Computer sowie einen in den Lieferwagen installierten Drucker und ein Ladegerät. Über den Computer, der Taschenrechnerformat hat und daher leicht tragbar ist, wickelte der Außendienstmitarbeiter sein gesamtes Tagesgeschäft ab, und zwar sowohl die Aufnahme, Abgabe und Rücknahme von Ware als auch die Abrechnung mit den Verkaufs- und Distributionsstellen. Neue Informationen, insbesondere Preise, wurden am Morgen in der Distributionsstelle in seinen Computer gespielt, die Tages-

1 Es sei erneut betont, daß mit dem Mapping angestrebt wird, sich auf die problemrelevanten Vorgänge und Beziehungen zu beschränken, einschließlich der Unterscheidung von Unternehmensteilen. Im Materialfluß wird beispielsweise nicht eingetragen, daß die Transportkartons zurückgenommen und wiederverwendet werden, daB nicht mehr frische Rückware (Stales) zurückfließt. Im Informationsfluß wird von Steuerungsdetails abstrahiert, auch von den Auseinandersetzungen an jedem Monatsende über die Ursachen von Schwund usw. 
endberichte mit jeder Einzelheit auf den Minicomputer der Distributionsstelle übertragen. (vgl. Abb. C.2.2)

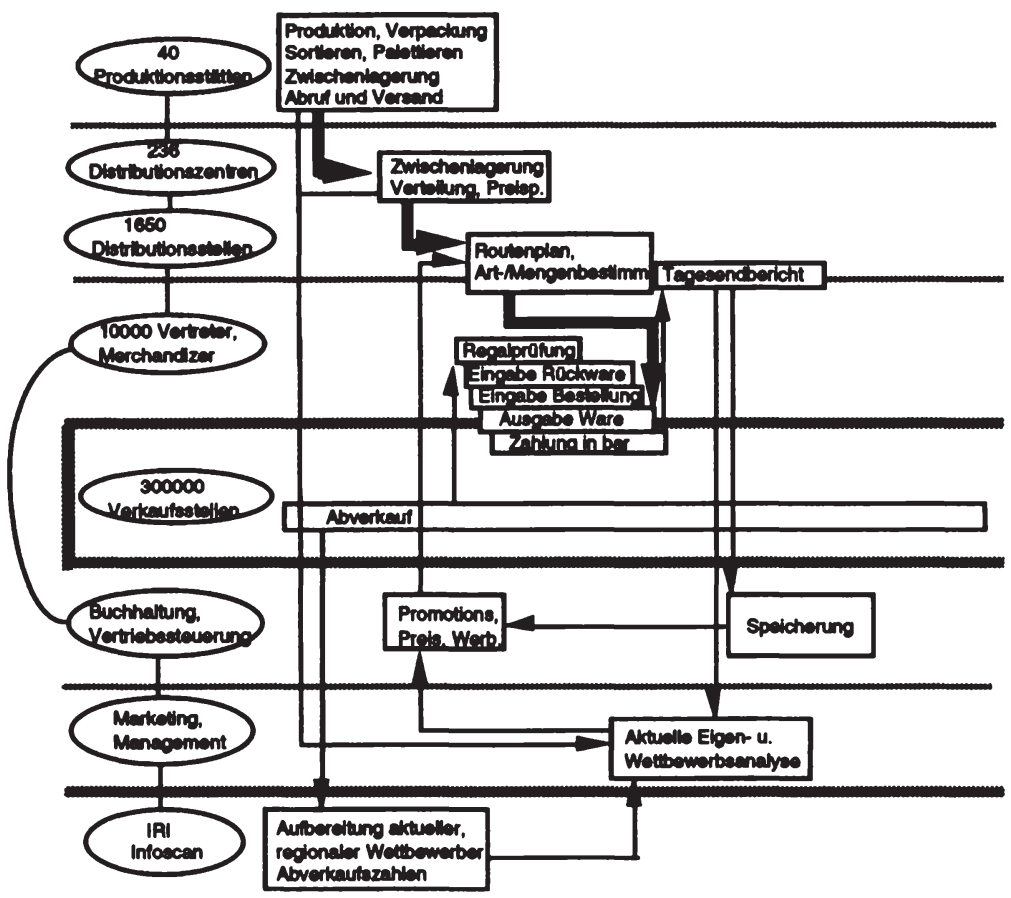

Abb. C.2.2: Frtb Ley, Inc.: Ausechnitu der KIS-gestotzien intecrativen Verbindungen

Damit entfielen einige besonders für den Außendienstmitarbeiter aufwendige administrative Koordinationsmechanismen ersatzlos, etwa die Beachtung komplizierter Preisregeln durch zahlreiche Promotions sowie die Erstellung und die Abstimmung des Tagesendberichts. Andere Schritte wurden wesentlich erleichtert und beschleunigt, wie die Erfassung von Rücknahmen, Bestellungen oder die Überprüfung des Soll-Lagerbestandes. Es kam kaum noch zu Schwund oder Erfassungs- bzw. Berechnungsfehlern. 
Das zweite Projekt folgte aus dem ersten. ${ }^{1}$ Durch den Hand-Held-Computer ist eine enge Integration der Geschäftsaktivitäten des Auftragszyklus selbst erreicht worden. Mit den täglich verfügbaren Daten gewann das Marketing von Frito Lay völlig neue Einsichten über das eigene Geschäft. Zentrales Problem war jedoch das Volumen dieser Daten. Als zweiter Schritt wurde daher ein umfassendes, unternehmensweites Management-Unterstützungssystem konzipiert. ${ }^{2}$ Es bestand aus einem Entscheidungsunterstützungssystem, einem Executive-Unterstützungssystem und einem unternehmensweiten Kommunikationssystem. Damit wurde ein Fülle von Daten abrufbar über die verschiedenen funktionalen Abteilungen, Regionen, befahrenen Routen, Absatzkänale, Produktionsqualität, PromotionenWirksamkeit, Konsumentendemographie, Lagerbestände und Schichterträge. Das System machte die eigenen Ergebnisse mit denen der Wettbewerber vergleichbar. Basis der Wettbewerberdaten waren extern bezogene Scannerdaten über 106 Regionen, die 20.000 Produkte, wöchentlich mit 110 verschiedenen Maßen enthielten. Sowohl die Unternehmensspitze als auch die Leiter der funktionalen Abteilungen erhielten Zugang zu rohen, unverarbeiteten Daten und konnten jene in vielfacher Hinsicht analysieren. Damit sah das Management den Markt nicht mehr als "Black box" an, sondern gewann ein besseres Verständnis für die Vorgänge im Markt. Unter dem Stichwort "Directed Decentralization" wurde es zudem möglich, eine im Geschäftsprozeß von Snacks zentrale Entscheidung zu dezentralisieren: den Tradeoff zwischen Marktanteil und Gewinn. Auf Basis der nunmehr verfügbaren, vollständigen, regionalen Informationsbasis konnten die Regionalmanager in den Distributionszentren die Konsequenzen von Preiszugeständnissen innerhalb kürzester Zeit erfahren. Damit wurde durch Integration mit KIS die Möglichkeit zur Dezentralisierung von Entscheidungen geschaffen, die aufgrund der zunehmenden Fragmentierung des Marktes angesichts kleiner, regionaler Wettbewerber dringend geboten schien.

1 Parallel wurde auch die Fertigung auf automatisierte Fertigungs- und Lagerstellen umgestellt. Dabei wurde ein zentrales Problem bei der Fertigung salziger Snacks, der Bruch, reduziert, indem die Anzahl nötiger Bewegungen von der Produktion bis zur Auslieferung erheblich gesenkt wurde. Zur Produktion bei Frito Lay vgl. Beck, L., Frito Lay, a.a.O., S. 78 - 81.

2 Vgl. dazu und am folgenden Applegate, L., Wishart, N., Frito Lay, Inc.: A Strategic Transition (C), Boston, Massachusetts, Harvard Business School, Case No. N9-190-071, 1990, S. 2 ff; zu technischen Details vgl. Francis, B., Frito Lays a New IS Bet, in: Datamation, Vol. 35, No. 4, 1989, S. 75 ff. 
Weitere Bestandteile der Projekte bei Frito Lay waren ein KIS-gestütztes Routing der Routen der Außendienstmitarbeiter, ${ }^{1}$ die Entscheidungsunterstützung der Auswertung der Wirkung von Promotions ${ }^{2}$ sowie ein System, mit dem die Unternehmensplanung von jährlichen Zyklen auf Trimester verkürzt werden sollte. ${ }^{3}$

Die Liste der genannten Ansatzpunkte KIS-gestützter Integration umfaßt eine Fülle verschiedener Prozesse bzw. Zyklen der Unternehmung, zu deren genauer Darstellung eine entstprechende, den Rahmen der Arbeit sprengende Fülle von Maps vorgelegt werden müßte. Die Grundlogik entspricht jedoch der in C.1. beschriebenen Strategie der internen Integration. Die interne Transaktionsintegration setzt beim Abbau der Schnittstellen an und verbindet nacheinandergelagerte Geschäftsaktivitäten. Durch Eingabe einer Information in einem vorgelagerten Unternehmensteil erhöht sich die Genauigkeit und die Geschwindigkeit der Übertragung zum nachgelagerten Unternehmensteil bei gleichzeitig sinkenden Informationskosten. Die interne Organisationsintegration setzt bei der Nutzung von Gleichartigkeiten an. Im Falle von Frito Lay wurden immaterielle horizontale Verflechtungen systematisch gebildet, indem gleichartige Informationen synergetisch verbunden wurden, z.B. Wettbewerberinformationen mit eigenen Informationen, deren Genauigkeit weit über Scannerdaten hinausging.

Frito Lay konnte nach Einführung der beschriebenen KIS erhebliche Positions- und Stabilitätsvorteile erreichen. In den Jahren nach Einführung des Hand-Held-Computers stiegen durch Positionsgewinne gegenüber den regionalen und den USA-weiten Anbietern Umsatz und Gewinn erheblich.4 Besonders hervorzuheben sind:

- Regalplatzgewinne: Auf Basis der gewonnen und hinzugefügten (Wettbewerber-) Daten konnte der Außendienst jedem einzelnen Händler vorrechnen, welchen Erfolg er mit Frito Lay und welchen

1 Vgl. Cassidy, W.B., Computerized Routing, Gaining the Competitive Edge, in: Fleet Owner, Big Fleet Edition, Vol. 85, No. 5, 1990, S. 82 - 84.

2 Vgl. Rothfeder, J., Bartimo, J., How Software is Making Food Sales a Piece of Cake, in: Business Week, 2. July 1990, S. 54.

3 Vgl. Applegate, L., Wishart, N., Frito Lay, Inc., a.a.O., S. 2.

4 Die "sichtbaren" Kosten des Hand-Held-Computer-Projekts können mit ca. \$40 Mio. beziffert werden, wobei die größten Anteile für die Beschaffung der Hand-HeldComputer, der Minicomputer in den Distributionsstellen und die Anpassung der Anwendungssoftware anfielen. Vgl. Applegate, L., Wishart, N., Frito Lay, Inc., a.a.O. 
er mit Konkurrenzprodukten erzielte. Mit dieser Argumentation und mit der für Verkaufsaktivitäten gewonnen Zeit gelang eine Ausdehnung der Regalfläche.

- Möglichkeit zum Mikromarketing: Im allgemeinen sah es das Management von Frito Lay, als wesentlichen Erfolg der Projekte an, daß sie ein Mikromarketing ermöglichten. ${ }^{1}$ Durch eine gezieltere Marktbearbeitung kleinerer, homogenerer Segmente bei gleichzeitig gesunkenen Differenzierungskosten (geringere Administrationskosten) sei damit ein Umsatzgewinn von $10-15 \%$ auch ohne neue Routen erzielt worden.

- Höhere Effizienz von Promotions: Angesichts der Analysemöglichkeit auf Basis realer eigener und Wettbewerberdaten hat die Promotionseffizienz um $30 \%$ zugenommen.

- Größere Distributionseffizienz: Dadurch wurden Kostenvorteile erreicht: Eine Ausweitung des Umsatzes von $\$ 3,2$ auf 4,2 Mrd. gelang ohne zusätzliches Einstellen von Außendienstmitarbeitern. ${ }^{2}$

Eine abschließende Beurteilung des zweiten, teilweise noch nicht abgeschlossenen Projekts war zum Erhebungszeitpunkt nicht möglich. Bedeutsam erscheint die Verbindung der beiden Strategien interner Integration, also der vertikal orientierten Transaktionsintegration und der horizontal ausgerichteten Organisationsintegration, die in anderen Fallstudien (noch) nicht beobachtet werden konnte. Strategische Integration hat die Wettbewerbsposition von Frito Lay erheblich verbessert, vor allem gegenüber den regionalen Anbietern. Auch die Dauerhaftigkeit dürfte gesichert sein, obwohl prinzipiell jede von Frito Lay vorgenommene Maßnahme imitierbar ist. Die Dauerhaftigkeit beruht hier auf der genauen Abstimmung von Grundgeschäft und KIS, auf dem Zusammenwirken der Fülle von Integrationsmaßnahmen, einem langjährig extrem hohen Servicegrad, ständiger Produkt- und Prozeßinnovationen sowie einer schnelleren, flacheren und effizienteren Organisation; hierzu trägt das KIS entscheidend bei. Dabei kann der Erfolg von Frito Lay nicht unabhängig von der Marktführerschaft gesehen werden. Weder der extrem hohe USA-weite Servicegrad noch die geschaffene Infrastruktur wären denkbar, verfügte Frito Lay nicht über das hinreichende Volumen zur Nutzung dieser Infrastruktur. Insofern hat der

1 Zum Mikromarketing vgl. Osborn, T.W., Micro Marketing into the Niches: The Eye of the Stranger, in: Marketing Communications, Vol. 12, No. 3, 1987, S. 57 - 72; Webber, J.C., Packaged Goods Marketing, a.a.O., S. RC3 - RC5; Lee, P.M., Micro-Marketing, a.a.O., S. 71 - 82; Schiller, Z., New Consumer, a.a.O., S. 54 - 62; Coleman, L.G., Go "Regional", a.a.O., S. 1,8 und 16 .

2 Die Entlohnung der Außendienstmitarbeiter erfolgt auf Kommissionsbasis mit Fixum. 
Einsatz von KIS eine bestehende starke Position noch verstärkt, aber sie keineswegs allein begründet.

\subsubsection{Transaktionsintegration}

Die Transaktionsintegration wirkt durch die Bildung vertikaler Binnenschnittstellen direkt auf die Wettbewerbsposition. Durch elektronische Unterstützung vertikaler Verflechtungen werden Doppelerfassungen vermieden, Vorgänge und die Informationsübermittlung beschleunigt, Übertragungsfehler reduziert und eine wesentlich engere Verzahnung nacheinandergelagerter Geschäftsaktivitäten möglich. So setzte Esab AB ein internes Bestellsystem ein, um europaweit Geschäftsaktivitäten zu verknüpfen. Esab AB, ein multinationaler Schweißgeräte- und Schweißbedarfhersteller mit einem Jahresumsatz von SEK 3,96 Mrd. war in 33 Tochtergesellschaften in 25 Ländern untergliedert, wovon einige reine Verkaufsgesellschaften sind. 1

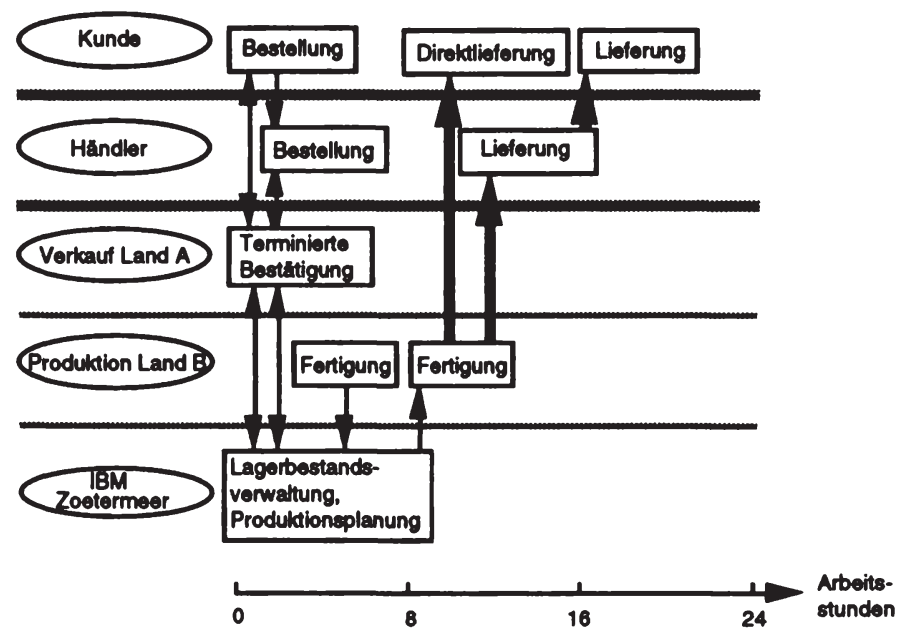

Abb. C.2.3 Transaktionsintegration: COSMOS System bei ESAB

1 Vgl. Mead, M., ESAB AB: Toughing it Out, Boston, Massachusetts, Harvard Business School, Case No. 9-188-005, 1988, S. 2 f. 
Abbildung C.2.3 enthält einen beispielhaften Auftragszyklus für Schweißbedarf von der Kundenbestellung bis zur Lieferung. Ein Kunde bestellte je nach Größe direkt oder über einen Händler. Das interne Verkaufspersonal der Tochterunternehmung konnte dabei in Echtzeit auf das System zugreifen und unmittelbar telefonisch Terminzusagen machen. Daraufhin erfolgte, falls kein Lagerbestand vorhanden war, die Fertigung am entsprechenden Produktionsort, von wo die Ware entweder über ein Zentrallager oder direkt an den Kunden geliefert wurde. Nach Einführung des Systems konnte Esab AB die Produktionskapazitäten konzentrieren und gleichzeitig Lagerbestände abbauen. Für viele Orte sind physische Lager durch "konzeptionelle Lager" ersetzt worden (d.h. verteilte Lager an wenigen, anderen Orten). ${ }^{1}$ Durch Bündelung des Transports wurde es möglich, von den Zuladungen der Ware anderer Firmen unabhängig zu disponieren und die gesamten Transportkosten um $10 \%$ zu senken. Neben den Kosten wurde der Zeitaufwand bei der Überschreitung von Zollgrenzen gesenkt. ${ }^{2}$ Gegenüber dem Kunden tritt Esab dabei nach wie vor mit einer multinationalen Strategie auf, d.h. als lokaler Anbieter in vielen Märkten, hält aber die Effizienz und Qualität einer gemeinsamen Produktion im Hintergrund vor. ${ }^{3}$ Insofern erlaubt die vertikale Verknüpfung des Auftragsprozesses gleichzeitig eine enge materielle horizontale Verflechtung gleichartiger Produktionsprozesse.

Die Transaktionsintegration kann in vielen Auftragsprozessen mit hohem Volumen beobachtet werden. So verflechten inzwischen fast alle Schnellrestaurants ihre Geschäftsaktivitäten zwischen Point-of-Sales, Distributionssystem und Zentralrechner und erzielen damit neben wichtigen Marktinformationen Zeit- und Kostenvorteile. ${ }^{4}$ Reiseunternehmen von Bus- bis zu Bahn- und Frachtunternehmen verflechten Verkauf, Vorreservierung, Zahlungsabwicklung und Einsatzplanung. ${ }^{5}$ Klassische Beispiele sind Ban-

1 Keen gibt für den Übergang auf verteilte Läger Lagerkostensenkungen von durchschnittlich $15-20 \%$ an, ohne allendings Quellen zu benennen. Vgl. Keen, P.G.W., Competing in Time. Using Telecommunications for Competitive Advantage, Cambridge, Massachusetts, 1988, S. 87.

2 Vgl. Mead, M., ESAB AB, a.a.O., S. 9.

3 Vgl. Konsynski, B.R., McFarlan, F.W., Information Partnerships - Shared Data, Shared Scale, in: Harvard Business Review, September-October, 1990, S. 118.

4 Vgl. Simson, C. von, Food Fight, in: Computerworld, Focus an Integration, 2. October 1989, S. 22 - 25.

5 Vgl. Kolodziej, S., En Route to Customer Satisfaction, in: Computerworld, Focus on Integration, June 5, 1989, S. 43 - 46; McGee, J.V., Vitale, M.R., Consolidated Rail Corporation: Railcar Identification Project, Boston, Massachusetts, Harvard Business School Case No. 9-188-082, Revised Version, 5/88, 1988. 
ken und Versicherungen für die Abwicklung von Zahlungsbewegungen aller Art, von Verträgen, des internen Berichtswesens und der Kreditsicherung sowie für die Unterstützung des Handels und der internen Kommunikation. ${ }^{1}$ Alle diese Beispiele sind von relativ geringer Unsicherheit und einer Abwicklungsorientierung gekennzeichnet.

Mit der Transaktionsintegration können direkte Positionsvorteile erzielt werden, die allerdings relativ leicht kopierbar und insoweit nicht dauerhaft sind. Die Dauerhaftigkeit von Posititionsvorteilen durch Transaktionsintegration wird daher nur in Abstimmung mit anderen Elementen der Wettbewerbsstrategie möglich sein, die sowohl aus dem Grundgeschäft stammen können wie aus einer Kombination der Transaktionsintegration mit der Organisationsintegration. Nicht zuletzt durch die Kombination einer starken Wettbewerbsposition mit der Transaktions- und der Organisationsintegration erschien Frito Lay als besonders erfolgreiches Beispiel strategischer Integration mit Informationstechnologie.

\subsubsection{Organisationsintegration}

Während die Transaktionsintegration vor allem auf die vertikale Binnenverknüpfung ausgerichtet ist, schafft die Organisationsintegration immaterielle horizontale Verflechtungen. Sinn der horizontalen Verflechtung ist die Verbindung gleichartiger Geschäftsaktivitäten bzw. bei Einsatz von KIS gleichartiger Informationen. Die Organisationsintegration ist anders als die Transaktionsintegration zumeist auf den Entwicklungs- und Managementprozeß gerichtet, weniger auf den Abwicklungsprozeß. Dabei wirkt die Organisationsintegration zumeist indirekt über die Verbesserung von Entscheidungsprozessen und Kommunikationsmöglichkeiten. In Abb. C.2.4 sind die Kernelemente des Managementprozesses der Xerox Corporation schematisch skizziert. ${ }^{2}$

Der Management-Prozeß bei Xerox Corp. war auf das Top-Management ausgerichtet. Einzelne Business Units bereiteten einen vier Seiten umfas-

1 Ein gutes Beispiel der Integration verschiedenster Prozesse ist Wells Fargo, Inc. Vgl. Feder, B.J., Getting the Electronics Just Right, in: The New York Times, June 4, 1989, Section 3, S. 1 und 8.

2 Vgl. zum folgenden Osborn, C.S., Applegate, L.M., Xerox Corporation: Executive Support Systems, Harvard Business School Case No. 9-189-134, 1988, S. 15 - 21. 
senden Bericht der Position im jeweiligen Markt vor, ergänzt mit Vorschlägen für Maßnahmen zur Verbesserung der Position. Diese Unterlage wurde im Corporate Office Management (COM) diskutiert, revidiert und um Kommentare ergänzt, eine zusammenfassende "Business Priorities List" erstellt. Diese wurde als "5. Seite" and die Business Unit zurückgesandt. Aus allen 5. Seiten wurde die "Direct Action List" des Präsidenten abgeleitet. Für einen Großteil der Kommunikation und

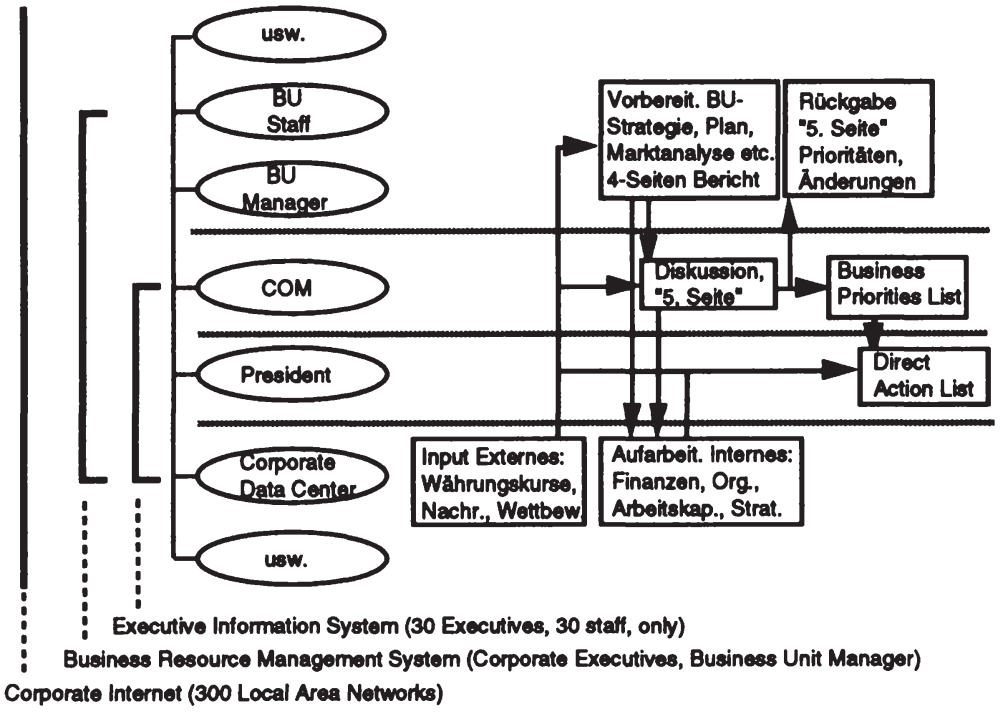

Abb. C.2.4 Überblick über den Managementprozesses bei Xerox Corp.

Information in jenem Proze $B$ wurde das mehrstufige KIS eingesetzt. Als Nutzen der Integration mittels KIS wurde angegeben, daß die Kommunikation beschleunigt und vertieft wurde. Weniger Top-Management-Zeit werde durch die "Feststellung des Status Quo" in Sitzungen verbraucht und so für Interpretation und Planung freigesetzt. ${ }^{1}$ Dazu verfügte Xerox auch über einen Raum für das "Executive Information System", der umfangreiche Präsentationsmöglichkeiten bot.

Statt der Ausrichtung auf das Top Management gibt es auch völlig andere Ansätze. So zielte das Computer Conferencing bei IBM auf die Kommuni-

1 Vgl. Osborn, C.S., Applegate, L.M., Xerox Corporation, a.a.O., S. 10 - 12. 
kation jenseits hierarchischer Kommunikationslinien. Prinzipiell konnte jeder Mitarbeiter von seinem Terminal eine Konferenz eröffnen und jeder andere sich einschalten. 1988 hatte IBM 300 Konferenzen, 100 davon waren "öffentlich", also für jeden Mitarbeiter zugänglich, 200 waren vom "Eigentümer" als privat deklariert. Die größte (und erste) Konferenz, IBMPC, hatte 40.000 Teilnehmer, 4.000 Themen und beanspruchte $5 \%$ der Kapazität des internen Leitungsnetzes bei IBM. Jeder der 385.000 Mitarbeiter konnte Fragen zum IBM Personal Computer an alle Teilnehmer der zugehörigen Konferenz richten. Als dem Autor das System präsentiert wurde, dauerte es nur wenige Sekunden, bis Antworten auf eine Frage aus Peru und Österreich in den USA vorlagen. ${ }^{1}$

Zwischen Executive-Informations-Systemen und Computerkonferenzen besteht ein Spektrum von Ansätzen, durch die Verbesserung innerbetrieblicher Kommunikation die innerorganisatorischen Prozesse zu verändern. ${ }^{2}$ Sehr weit geht die Reorganisation der Finanzdienstleistungen bei General Electric, Canada. Als Ziel einer Organisationsintegrationsstrategie gab der Leiter des Bereichs an, gleichartige Arbeiten zusammenzulegen, den Ablauf der Geschäftsaktivitäten $z u$ analysieren und $z u$ vereinfachen sowie eine flachere, reagiblere Organisation zu schaffen. Hierzu sollte insbesondere der Einsatz von KIS beitragen. Die vormals 360 Mitarbeiter umfassende Abteilung wurde um $40 \%$ gestrafft, zwei hierachische Ebenen ersatzlos abgeschafft und durch KIS-gestützte "Self Managing Groups" ersetzt. Die formale interne und externe Kommunikation wurde weitgehend auf Electronic Mail umgestellt. ${ }^{3}$ Die Umstellung kann als ein konsequentes Umsetzen der Nutzung von Gleichartigkeiten und des Abbaus von Schnittstellen durch den Einsatz von Informationstechnologie interpretiert werden, wobei das KIS zahlreiche administrative und strukturelle Koordinationsmechanismen substituiert hat, während die personalen Koordinationsmechanismen stärker betont wurden.

1 Zum IBM Conferencing System vgl. Chess, D.M., Colishaw, M.F., Conferencing, a.a.O., S. 138 - 153; Smith, H.J., Applegate, L.M., IBM Computer Conferencing, Boston, Massachusetts, Harvard Business School Case No. 9-188-039, 1988.

2 Andere Beispiele finden sich bei Keen, P.G.W., Competing in Time, a.a.O., S. 83 ff; Rockart, J.F., DeLong, D., Executive Support Systems, a.a.O., S. 65 ff.

3 Vgl. Applegate, L.M., Cash, J.I., GE Canada: Designing a New Organization, Boston, Massachusetts, Harvard Business School Case No. 9-189-138, Rev. 11/89, 1989, S. 3 - 6. Die Implementierung bestätigte die von Drucker identifizierten Probleme einer flachen Organisation der Zukunft, nämlich die Anreiz- und Lohnsysteme und das Problem mangelnder Entwicklungsmöglichkeiten für Managementaufgaben. Vgl. Drucker, P.F., New Organization, a.a.O., S. 50 - 52. 
Neben der reinen Kommunikationsunterstützung ist die Entscheidungsunterstützung ein wichtiger Zweck einer Organisationsintegrationsstrategie mit KIS. ${ }^{1}$ Beispielsweise nutzte Philips 66, ein in mehreren Öl- und Ölnahen-Branchen angesiedeltes Verarbeitungs- und Distributionsunternehmen, ein umfassendes Executive-Unterstützungssystem. Neben der Übermittlung externer Nachrichten und interner Statusberichte bot das System die Möglichkeit, verschiedenste Analysen und Simulationen benutzerfreundlich auszuführen und darzustellen. Als direkten Nutzen des Systems wurde eine Reduktion von Lagerbeständen um durchschnittlich 1 Mio. Barrel Rohöl angegeben, zudem eine wesentlich flexiblere und den Marktverhältnissen nähere Preispolitik im Benzinmarkt. Vormals dauerte es mit einem papiergestützten System Tage, bis eine Preisänderung im gesamten Tankstellennetz implementiert werden konnte. Ein strukturelles Mispricing bei Philips 66 von nur einem Cent zöge eine Gewinnminderung von jährlich $\$ 40$ Mio. nach sich.

Die Integration durch Entscheidungs-Unterstützungssysteme ist auch auf Gruppen ausdehnbar. Der Elektrokonzern Westinghouse setzte ein Gruppenentscheidungs-Unterstützungssystem, welches mit einem elektronischen Abstimmungssystem kombiniert war, zur Kapitalbudgetierung ein. ${ }^{2}$ Eine aus Entscheidungsträgern verschiedener Unternehmensteile zusammengesetzte Gruppe entschied je Sitzung über 200 Projektvorschläge nach sechs Kriterien in elektronischer geheimer Abstimmung. Die Gruppe traf dabei eine Vorauswahl, die tatsächliche Entscheidungskompetenz hing vom Projekt ab. Das Abstimmungsverhalten und die Kommentare wurden den Investitionsvorschlagenden und den weiteren an der Entscheidung Beteiligten übermittelt. Mit diesem Verfahren konnten sehr effizient und verdichtet die Perspektiven unterschiedlichster Unternehmensteile eingeholt werden, so daß verbesserungsbedürftige Vorschläge aus dem Prozeß eliminiert werden konnten, bevor sie in (zeitlich) aufwendigere Stufen des Bewertungs- und Entscheidungsprozesses gelangten. Die Anonymität der elektronischen Abstimmung, die Geschwindigkeit der Abwicklung und die Möglichkeit der Differenzierung nach mehreren Kriterien veränderten den

1 Vgl. King, W.R., Decision Support Systems, a.a.O., S. 428 ff.

2 Vgl. zum folgenden Applegate, L.M., Hertenstein, J.H., Wishart, N., Adonizio, M., Westinghouse Electric Corp.: Automating the Capital Budget Process (A), mit Supplements 1 - 3, Boston, Massachusetts, Harvard Business School Cases No. 9-189-119; 9-189-122, 1989. 
Entscheidungsprozeß erheblich. Sie schufen die Möglichkeit, schon in frühen Phasen bei einer Beratung von Investitionsvorschlägen die Meinung verschiedener Unternehmensteile einzuholen. Insofern stellten sie eine wichtige horizontale Verflechtung dar.

Direkte Auswirkungen der Organistionsintegration durch KIS waren die Substitution administrativer und struktureller Koordinationsmechanismen, eine Beschleunigung der Kommunikation, die Verbesserung der Genauigkeit und Präsentation von Daten, die Möglichkeit zu Ad-hoc-Analysen, die verbesserte informale Kommunikation, verbesserte Teamkoordination, die Veränderung des "mentalen Modells" der Unternehmung durch breiteren Zugang zu rohen, unaggregierten Daten sowie zu externen Daten und zu Tests und Simulationen alternativer Annahmen und Vorgehensweisen. 1

Damit beeinflußt die Organisationsintegrationsstrategie nur indirekt die Kosten und den Nutzen der Integration, sie kann jedoch erhebliche Auswirkungen haben. Sinkende Informations- und damit Administrationskosten reduzieren das Agencyproblem und eröffnen die Möglichkeit für dezentrale Entscheidungen. ${ }^{2}$ Der Einsatz von Informationstechnologie beschleunigt den Managementprozeß, neben dem unmittelbaren Zeitvorteil kann dies bei Relevanz von "First-Mover"-Vorteilen auch Kosten- und Stabilitätsvorteile bringen. Durch eine verbesserte Informationsbasis dürfte auch das Verteilungsproblem und intern die Nichtausnutzung des Erfolgspotentials aus dem Einsatz von Produktionsfaktoren reduziert werden. Zudem wirkt die KIS-gestützte Organisationsintegration in hohem Maße auf die Flexibilität, indem der Managementprozeß beschleunigt und das "mentale Modell" der Unternehmung verbessert wird. Damit erhöht sich die Lerngeschwindigkeit, die Spreizung und die Beobachtbarkeit definierter Zukunftszustände, also der Wert der strategischen Flexibilität. ${ }^{3}$

1 Vgl. King, W.R., Decision Support Systems, a.a.O., S. 428 ff; Rockart, J.F., DeLong, D., Executive Support Systems, a.a.O., S. 94 ff.

2 Vgl. auch Keen, P.G.W., Competing in Time, a.a.O., S. 83 ff.

3 Umgekehrt kann mit gleichem Ergebnis anhand sinkender "unobserved Heterogeneity" argumentiert werden, vgl. Abschnitt B. 1.3.2.2.2. 


\subsection{Geschäftsprozeßintegration}

Die Geschäftsprozeßintegration umfaßt im Vergleich zur internen Integration den gesamten Geschäftsprozeß. Eine Unternehmung, die die Abwicklung ihres gesamten Geschäfts fundamental und umfassend auf die Nutzung von KIS umstellt, kann auch als KIS-basierte Unternehmung bezeichnet werden. Schwerpunkt der GeschäftsprozeBintegration ist die Bildung elektronischer vertikaler Verknüpfungen. Im Vergleich zur Geschäftsnetzwerkintegration bleibt der Integrationsbereich dabei auf die (kontraktuellen) Grenzen der Unternehmung begrenzt. ${ }^{1}$

\subsubsection{Geschäftsprozeßintegration bei Batterymarch}

"My idea of the future money management organization is a few senior people and one big machine", 2 faßte Dean LeBaron, Präsident der Bostoner Vermögensverwaltungsfirma Batterymarch Financial Management, seine Vision des Vermögensverwaltungsgeschäfts zusammen. Batterymarch verwaltete 1984 Aktiendepots von insgesamt \$10.7 Mrd. Die 116 Kunden waren institutionelle Anleger verschiedener Art und Größe, vor allem mittelgroße Pensionsfonds mit Volumina von je $\$ 50$ Mio. und mehr. Batterymarch verwaltete das Gesamtvolumen mit 34 Mitarbeitern und erzielte ein Provisionseinkommen von $\$ \mathbf{8 8 0 . 0 0 0}$ pro Mitarbeiter. ${ }^{3}$

\subsubsection{KIS-Einsatz im Geschäftsprozeß der Vermögensverwaltung}

Die Anlagestrategie von Batterymarch wurde als "contrarian, value-oriented, opportunistic and conservative" bezeichnet. 4 Auf den Inhalt der daraus

1 Hier besteht eine Grauzone, was bei relationalen Kontrakten als kontraktuelle Grenze anzusehen ist: Die Grenze der Geschäftsprozeßintegration ist bei den firmenorientierten, engen relationalen Verträgen al ziehen. Demgegenüber wird ein gemeinsames Kommunikationssystem zwischen Unternehmen, die kontraktuell mu durch marktorientierte relationale Verträge integriert sind, bereits als Geschäftsnetzwerkintegration angesehen (z.B. elektronische Verknüpfungen zwischen Pharmagroßhandel und Apotheke, die rechtlich nur durch langfristige Lieferverträge integriert sind).

2 O.V., The LeBaron Phenomenon, in: Institutional Investor, August, 1985, S. 107.

3 Vgl. o.V., LeBaron, a.a.O. , S. 107.

4 Clarke, R.F., The Application of Information Technology in an Investment Management Firm, Unpublished Thesis, Massachusetts Institute of Technology, Sloan School of Management, Cambridge, Massachusetts, 1985, S. 53. 
folgenden Anlagestrategie sei hier nicht eingegangen. Es sei lediglich festgehalten, daß erstens der Anlagephilosophie die Auffassung zugrunde liegt, daß es durch aktive Gestaltung des Portfolios möglich sei, einen Überschuß über dem Marktdurchschnitt zu erzielen. ${ }^{1}$ Zweitens erlaube die Strategie eine Formulierung von Regeln, nach denen Kauf- und Verkaufsentscheidungen zu treffen sind.

Batterymarch faßte diese Regeln in fünf Modelle (Entscheidungs-Unterstützungssysteme), ${ }^{2}$ die von zentraler Bedeutung für das Investment Management bei Batterymarch waren. Die Regeln wurden von 11 Investment Managern, die außerdem für Marketing- und Vertrieb zuständig waren, ständig fortentwickelt. Batterymarch beobachtete innerhalb der USA eine Grundgesamtheit von 3000 Werten, wobei als Input in die Modelle $\mathbf{4 0 0}$ Datenfelder je Wert quartalsweise für die jeweils abgelaufenen vergangen 15 Jahre betrachtet wurden. Die Daten wurden auf Bändern von verschiedenen Anbietern erworben. ${ }^{3}$

Monatlich wurde auf Basis der Modelle ein neuer Optimierungslauf über die 3000 Werte vorgenommen. Der eigentliche tägliche Anlageprozeß basierte auf diesen monatlichen Optimierungen, den Tageskursen und den jeweiligen Preisen sowie den Handelszahlen des Vortags. Ein Mapping des Tageszyklus bei Batterymarch ist innerhalb des Kastens in Abbildung C.2.5 angegeben.4

1 Es ist die Mindestanforderung an jede "aktive" Anlagestrategie für ein Aktienportfolio, daß diese zu einem Überschuß über dem Marktdurchschnitt, zzgl. den durch die Strategie verursachten Transaktionskosten, führt. Denn ansonsten könnte das Portfolio passiv in den Marktdurchschnitt investiert werden.

2 Die Modelle sind (1) Financial Tension, ein Modell zur Identifikation möglicher Übernahmekandidaten, (2) Segment, ein Modell zur Bewertung von Unternehmen anhand des Ertrags der einzelnen Geschäftsfelder, (3) Unpopular Industries, ein Modell zur Ermittlung derzeit möglichst unbeliebter Werte, (4) Low Expectations, ein Modell zur Erfassung der Analystenerwartungen und (5) Neglected Stocks, ein Modell zur Ermittlung won Werten, die von nur wenigen Analysten verfolgt werden. Vgl. Dhebar, A.S., Warbelow, A., Ostofsky, K. , Batterymarch Financial Management: Information Systems and Technology, Harvard Business School Case No. 9-188-013, Boston, 1987, S. 15. Hinter der Formulierung dieser Modelle steht jeweils die Erwartung, daB z.B. die Anlage in die identifizierten Aktien den Marktdurchschnitt schlägt, also z.B. die Anlage in nach "herrschender" Meinung unbeliebte Aktien.

3 Compustat, Value Line, Vickers, IBES, Fond und MSCI. Vgl. Dhebar, A.S., Warbelow, A., Ostofsky, K., Batterymarch Financial Management: Information Systems and Technology, Harvard Business School Case No. 9-188-013, Boston 1987, S. 16 - 17.

4 Vgl. Dhebar, A.S., Warbelow, A., Ostofsky, K., Batterymarch Financial Management, a.a.O., S. 16 - 17. Der Zyklus wird nach dem Stand vom Juni 1987 beschrieben. Bei der Darstellung fällt die Unterscheidung von "Unternehmensteilen" schwer - Batterymarch läßt sich kaum in "physische" Teile untergliedern. 
Um 18 Uhr wurde aus dem abgeschlossenen Handelstag und der Bewertung aller beobachteten Aktien aus den Modellen für jedes einzelne der 116

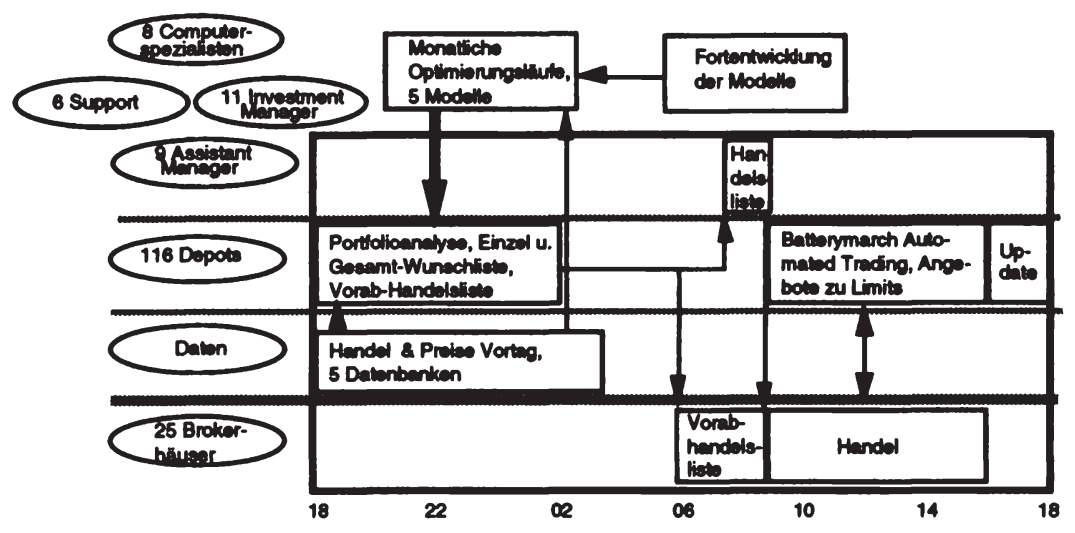

Abb. C.2.5: GeschutsprozeBintegration bei Bettorymarch Financial Maneoement

Portfolios eine "Wunschliste" für Änderungen ermittelt. Diese enthielt täglich durchschnittlich 4000 einzelne Transaktionen, welche über die Gesamtzahl der Portfolios auf 140 konsolidiert wurde. Jene Liste wurde am Morgen um 6 Uhr "vorab" veröffentlicht, indem Broker sich telefonisch in den Rechner von Batterymarch oder über das GTE-Telenet einwählen konnten. ${ }^{1}$ Eine Gruppe von Assistenzmanagern prüfte dann diese Vorabliste daraufhin, ob (noch) nicht in den Computer eingegebene Wünsche oder Restriktionen von Kunden durch die vorgeschlagenen Transaktionen verletzt würden, und gab die Liste um 9:30 frei zum Handel. Dabei wurde den Brokern elektronisch ein Abschluß zu einem Limitpreis angeboten. Der erste, der das Angebot akzeptierte, bekam das Geschäft. Durchschnittlich wurden 90 der 140 Aufträge tatsächlich gehandelt, Batterymarch zog Limits oft über lange Zeiträume hin. Die Abwicklung und Zahlung gehandelter Positionen erfolgte elektronisch. Am Ende des Handelstages wurden die Veränderungen in die einzelnen Depots umgesetzt.

1 Es handelt sich dabei um ganz gewöhliche elektronische Kommunikation, die nicht als technische Integration der Broker mit Batterymarch angesehen werden muB; andernfalls wäre Batterymarch ein Fall von Geschäftsnetzwerkintegration. Konzeptuell ist Batterymarch jedoch eindeutig der GeschäftsprozeBintegration zuzuondnen, denn intern und nicht im Telefonaustausch mit den Brokern wird der Wettbewerbsvorteil erreicht. 


\subsubsection{Dauerhafter Vorteil für Batterymarch?}

Batterymarch ist eine einzige vertikale Verflechtung. Die vertikale Verflechtung des Geschäftsprozesses mit KIS ist so weitgehend, daß im täglichen, operativen Geschäft ein Integrationsproblem im wesentlichen nicht mehr existiert. Dies wird begünstigt durch die Möglichkeit, den Geschäftsproze $B$ an die Strukturiertheitsanforderungen von Abwicklungssystemen nicht nur anzupassen, sondern den Geschäftsprozeß geradezu darauf aufzubauen. Die "Produktionsfunktion" dieses Prozesses sind dabei die Entscheidungsunterstützungssysteme, die die Basis jeglicher Anlageentscheidung darstellen und in die Abwicklungssysteme eingebunden wurden. Gleichzeitig erlaubt elektronische Kommunikation mit Brokerhäusern einen automatisierten Handel. Aus der vertikalen Verknüpfung ergibt sich zudem eine horizontale Verflechtung. Der Bewertungsprozeß jedes einzelnen der 116 Depots ist, abgesehen von individuellen Restriktionen, prinzipiell gleichartig.

Batterymarch war außerordentlich erfolgreich. In 11 von 17 Jahren konnte der am Standard und Poors Index gemessenene Marktdurchschnitt übertroffen werden, in fünf Jahren wurde der Index unterschritten, einmal ergab sich die gleiche Entwicklung. Der Anlageerfolg ist auf die Anlagestrategie zurückzuführen, die sich für jene Jahre als richtig herausstellte. ${ }^{1}$ Der Einsatz von KIS zur strategischen Integration trug jedoch erheblich zur Wettbewerbsposition von Batterymarch bei, insbesondere zur Qualitätsposition.

Das Nutzungskriterium der Qualität von Anlageverwaltern ist der zukünftige Anlageerfolg. Dieser ist jedoch nicht ex ante bestimmbar. Insofern entscheiden Signalkriterien über die Zuweisung von Anlagemitteln auf einen Verwalter. Diese sind, neben dem Anlageerfolg in der Vergangenheit, (1) ein klar definierter Ansatz, wie der Marktdurchschnitt übertroffen werden soll, (2) ein konsequentes Umsetzen dieses Ansatzes, (3) eine klare, deutlich formulierte Disziplin für Verkaufsentscheidungen und (4) die Kontinuität des Personals. ${ }^{2}$ Batterymarch weist in allen Kriterien wesentliche Wettbe-

1 Diese Anlagestrategie selbst soll hier allerdings nicht beurteilt werden.

2 Basis dieser Aussagen ist eine empirische Studie, die von AT\&T in Auftrag gegeben wurde. Vgl. o.V., ATT Finds Key Elements for Managers, in: Pensions \& Investment Age, 13. Oktober 1975, S. 37 ; vgl. auch Clarke, R.F., Application of Information Technology a.a.O. 
werbsvorteile auf. Die außerdem vorhandenen Kosten- und Zeitvorteile sind offensichtlich. So kann angenommen werden, daß eine vergleichbare Vermögensverwaltung bei traditionellem Vorgehen ca. 150 Mitarbeiter anstellen müßte, ca. 30 Professionals und 120 Support-Mitarbeiter. Aber auch in anderen Bereichen ist die Kostenposition vorteilhaft, so zahlte Batterymarch durch automatisiertes Trading nur 2 Cents pro Aktie an Brokerspesen im Vergleich zu 8 Cents pro Aktie im Branchendurchschnitt.

Die Dauerhaftigkeit des Wettbewerbsvorteils dürfte jedoch gefährdet sein. Batterymarch hat nahezu keine Flexibilitätsoption, ist vielmehr von einem starren Faktor abhängig. Die Anlagestrategie ("Contrarian") hatte sich zwar über Jahre als erfolgreich erwiesen. Dieser Erfolg ist jedoch vom Verhalten anderer Marktteilnehmer abhängig. Batterymarch kann jedoch die Anlagestrategie nicht beliebig verändern, insbesondere nicht auf Strategien setzen, die nicht mit Entscheidungsunterstützungssystemen abbildbar sind. ${ }^{1}$ Denn dazu müßte der Geschäftsprozeß modifiziert werden (z.B. auf "herkömmliche" analystenbasierte Einzelkundenbetreuung). Diese Veränderung würde nicht mehr die vom Kunden wahrgenommenen Stärken von Batterymarch nutzen - Mittelabflüsse würden drohen. Würde sich demgegenüber herausstellen, daß eine KIS-gestützte "Contrarian"-Strategie nachhaltige Erfolge zeitigt, wäre nicht die Flexibilität, sondern die Stabilität die Ursache mangelnder Dauerhaftigkeit: Der Ansatz ist prinzipiell sehr leicht kopierbar und kann schon binnen weniger Jahre vielfach imitiert werden. Dann ist der Mittelzufluß begrenzt bzw. setzt ein Mittelabfluß ein. Somit ist die Dauerhaftigkeit der Wettbewerbsposition durch ein Dilemma zwischen Flexibilität und Stabilität gefährdet.

Batterymarch ist ein sehr fragiles strategisches Commitment eingegangen. Die Logik des gesamten Geschäftsprozesses ist auf die Funktion eines starren, schwer veränderlichen Faktors aufgebaut. Verallgemeinernd kann geschlossen werden, daß die Umstellung des gesamten Geschäftsprozesses auf die strategische Integration durch KIS erhebliche Auswirkungen auf die Wettbewerbsposition haben kann, wenn ein "Fit" des Geschäfts und der Strukturierungsanforderung von Abwicklungs- und Managementsupport-

1 Ende 1987 soll Batterymarch begonnen haben, auch Expertensysteme zur Konstruktion der einzelnen Portfolios einzusetzen, die es erlauben, ein weit komplexeres Set portfoliospezifischer Restriktionen zu berücksichtigen. Vgl. Schmerken, I., Expert Systems Churns Many Happy Returns, in: Wall Street Computer Review, Vol. 5, No. 3, 1987, S. 14 - 17. 
systemen herstellbar ist. Dabei ergeben sich jedoch erhebliche Dauerhaftigkeitsrisiken bzw. ein Dauerhaftigkeitsdilemma entweder durch Flexibilitätsoder durch Stabilitätsprobleme. Flexibilitätsprobleme werden besonders dann auftreten, wenn ein KIS den ganzen Geschäftsprozeß trägt und die Erfüllbarkeit der Strukturierungsanforderung an die Geschäftsaktivitäten im Zeitablauf schwankt. Die Strukturierungsanforderung ist eine Funktion der Art des eingesetzten KIS. Insofern wird ein von Abwicklungssystemen getragener Geschäftsprozeß tendenziell besonders hohe Flexibilitätsprobleme nach sich ziehen. Liegt kein Flexibilitätsproblem vor und ist der Einsatz des Systems erfolgreich, wird sich demgegenüber ein Stabilitätsproblem durch Imitation ergeben. Dem kann bei KIS-Einsatz nur begegnet werden durch stetige Sicherung des technischen Vorsprungs oder durch Aufbau struktureller Mobilitätsbarrieren, die auf anderen Faktoren als dem KISEinsatz beruhen.

\subsubsection{Geschäftsprozeßintegration: Ansätze und Wirkungsweisen}

Der Einsatz von KIS beeinflußt den Geschäftsprozeß einer KIS-basierten Unternehmung umfassend und maßgeblich. Batterymarch kann nach Umfang und Bedeutung als Vertreter der "reinen Form" der Geschäftsprozeßintegration gelten. Eine derart konsequente integrationsstrategische Ausrichtung ist unter anderem deshalb möglich, weil die Vermögensverwaltung keinen physischen Materialfluß erfordert. Auch weniger weitgehende elektronische vertikale Verknüpfungen sind als Geschäftsprozeßintegration anzusehen, wenn sie der Logik eines Geschäftsprozesses wesentlich zugrunde liegen. Beispiele hierfür sind in so verschiedenen Branchen wie Aufzugservice, Leuchtkörper, Electronic Banking, Informationsraffinerien ${ }^{1}$ oder Frischbackwaren zu finden. Das Muster der Geschäftsprozeßintegration ist in diesen so verschiedenen Fällen sehr ähnlich. Durch eine konsequente Abstimmung der Geschäftsaktivitäten mit dem jeweiligen Strukturierungserfordernis von Abwicklungs-, Informations- und gelegentlich Entscheidungs-Unterstützungssystemen werden vertikale Verknüpfungen zum Abbau hochvolumiger Geschäftsprozesse geschaffen und Automati-

1 Den Begriff der Informationsraffinierie entlehnen Clippinger und Konsynski aus dem Vergleich am Cracken von Erdöl. Für eine überblickshafte Darstellung der technischen Einzelheiten der Weiterverarbeitung von Informationen mit KIS vgl. Clippinger, J.H., Konsynski, B.R., Information Refineries, Author's Draft, to be published in: Computerworld, August 1989, S. $73-77$. 
sierungspotentiale für die horizontale Verflechtung gleichartiger Aktivitäten genutzt.

Mrs. Fields Cookies, eine Kette von ca. 670 Läden, bot 1988 (im wesentlichen in 416 Läden) Frischkekse, Brownies und Muffins an. ${ }^{1}$ Der tägliche Geschäftsprozeß im einzelnen Laden begann damit, die Verbindung zwischen dezentralem PC und dem Zentralrechner aufzunehmen. Nach Eingabe verschiedener, relevanter lokaler Informationen (Schul- vs. Ferien- vs. Sonntag, Wetter etc.) wurde modellgestützt bestimmt, welche Frischteige in welcher Menge herzustellen waren. Der laufende Abverkauf der 14 Kekssorten wurde registriert. Lag die Kundenzahl unter der erwarteten, wurde automatisch eine Reihe von Vorschlägen an das am Umsatz beteiligte Personal gemacht, z.B. die Auslage von Proben. Lag der Verkauf pro Kunde unter den Erwartungen, wurde z.B. ein stärkeres Cross-Selling empfohlen. Neben dem unmittelbaren Verkaufsproze $B$ wurde insbesondere das Einhalten von Qualitätsstandards unterstützt (Vernichtung von Rohteig nach einer bestimmten Zeit) sowie die Personaleinsatzplanung und die Lohnbuchhaltung. Selbst Personalinterviews wurden interaktiv unterstützt und ein Entscheidungsvorschlag gegeben. Mrs. Fields Cookies erzielte 1988 einen Umsatz von $\$ 133$ Mio. mit 8000 Angestellten, davon 105 Gebietsmanager und 17 Regionaldirektoren.

Die Organisationsstruktur war mit vier Ebenen außerordentlich flach. Nur 140 Mitarbeiter arbeiteten in der Konzernleitung, darunter Controller, die täglich den Geschäftsverlauf von je 35 - 75 Läden verfolgten. Jeder Angestellte hatte zu jedem anderen, einschließlich CEO Debby Fields, Zugang via Electronic Mail. Der gesamte operative Geschäftsprozeß wurde durch verschiedene KIS unterstützt. Dies erlaubte es, Qualitätsstandards einzuhalten in einer von hoher Personalfluktuation gekennzeichneten Branche. Durch eine präzise Personaleinsatzplanung und extrem geringe Overhead-Kosten konnte Mrs. Fields Cookies einen Kostenvorteil realisieren. Ein weitgehender Abbau zahlreicher vertikaler Schnittstellen in vertikaler Richtung führte zu einer hohen Effizienz der Organisation, die Managementkapazität zu Wachstum und Akquisitionen freisetzte. Obwohl der KIS-Einsatz beträchtliche Positionsvorteile ermöglicht, liegt hier kein KIS-basierter Stabili-

1 Zum folgenden vgl. Ostrofsky, K., Mrs. Fields Cookjes, Boston, Massachusetts, Harvard Business School Case No. 9-189-056, Rev. 25-07-89, 1989, S. 1 ff; Walton, R.E., Up and Running, Integrating Information Technology and the Organization, Boston, Massachusetts, 1989, S. 34 ff. 
tätsvorteil vor. Prinzipiell sind die organisatorischen Rezepte von Mrs. Fields leichter imitierbar als diejenigen des Backprozesses.

In einem wesentlich komplexeren mit KIS integrierten Geschäftsprozeß stand Lithonia Lighting. Mit einem Umsatz von \$ 559 Mio. war das Unternehmen Marktführer im US-Markt für Leuchtkörper. Identisch war jedoch die Art Integration: die vertikale Verknüpfung aller wesentlichen $\mathrm{Ge}$ schäftsaktivitäten von der Konstruktion, der Wirtschaftlichkeitsanlyse bis zur Produktionsplanung, der Produktion und Lieferung. Dazu wurde eine Fülle verbundener KIS eingesetzt. ${ }^{1}$ Die Geschäftsprozeßintegration hat $\mathrm{zu}$ erheblichen Positionsvorteilen, insbesondere Zeitvorteile bei Auftragsfertigung und Kostenvorteile bei Serienfertigung, geführt und die Marktführerschaft mit begründet. Die Stabilität der Position ist jedoch nicht in erster Linie auf die Geschäftsprozeßintegration zurückzuführen, sondern vielmehr auf ein von den kleineren Anbietern nicht kopierbares Sortiment. Durch eine hohe Sortimentsbreite waren die Beschaffungskosten industrieller Kunden bei Lithonia geringer als bei den auf Teilsegmente spezialisierten Konkurrenten.

Die Stabilitätsproblematik der Geschäftsprozeßintegration kann gut aufgezeigt werden am Beispiel des Einsatzes automatischer Bankschalter (ATM = Automatic Teller Machine). In der einschlägigen Literatur wird es vielfach als geradezu rätselhaft bezeichnet, warum nach der frühen Euphorie der ATMs der dauerhafte Erfolg ausblieb. ${ }^{2}$ In zeitablaufbezogener Perpektive ist dies jedoch keineswegs überraschend. ATMs sind an Bankgebäuden, Kios-

1 Auf eine breite Darstellung wird aus Raumgründen verzichtet und auf die Literatur verwiesen. Vgl. Kolodziej, S., The Glow of Success, in: Computerworld, Focus on Integration, October 2, 1989, S. 26 - 30. Hofman, D.J., Rockart, J.F., Lithonia Ligthing: Case Study. Working Paper No. 201, Massachusetts Institute of Technology, Center for Information Systems Research, Cambridge, Massachusetts, 1989.

2 Die folgende Auswahl von Zitaten aus der bankfachlichen Literatur mag dies belegen: "Automated teller machines (ATM) are not attracting customers the way they used to": Schmitzer, I.L., ATM's at Maturity, in: Texas Banking, Vol. 77, No. 12, December, 1988, S. 11; "Facing a New ATM Reality": Silber, K., Facing a New ATM Reality, in: Bank Systems \& Technology, Vol. 26, No. 12, December 1989, S. 26; "ATMs at Maturity": Schmitzer, I.L., ATM's at Maturity, a.a.O., S. 11; "ATM Results Unproven": Jaben, J., ATM Results Unproven, in: United States Banker, Vol. 99, No. 12, December 1988, S. 36), "Many Bankers are wondering if the millions of dollars invested in installing automated teller machines (ATM) have been worth it": Jaben, J., ATM Results, a.a.O., S. 36, "Despite the popularity for automated teller machines (ATM), banks no longer are eager to place them in every available location": Fink, R.B., Reevaluating ATM Strategy, in: United States Banker, Vol. 98, No. 4, April 1989, S. 60; " Bankers find it difficult to quantify the harddollar impact of an automated teller machine": Bond, C., ATMs Pay Out, but Do They Pay Off?, in: Bankers Monthly, Vol. 106, No. 12, December 1989, S. 40. 
ken, in Supermärkten, Tankstellen oder anderen verkehrsträchtigen Stellen aufgestellte Automaten, über die Bankkunden täglich 24 Stunden lang prinzipiell sämtliche Banktransaktionen des Mengengeschäfts ausführen können, ${ }^{1}$ einschließlich des Einholens von Informationen, z.B. über Börsenkurse. Ende 1989 waren in den USA 87.136 ATMs installiert. ${ }^{2}$

Mit der Einführung von ATMs wurde einerseits angestrebt, durch vertikale Verknüpfung der Kundenschnittstelle mit nahezu jeder operativen Abteilung der Bank den Geschäftsprozeß im Mengengeschäft wesentlich zu vereinfachen, zu beschleunigen und den wachsenden Kosten, vor allem den Personalkosten, zu begegnen. Außerdem sollte die relative Qualitätsposition verbessert werden, indem ständige Verfügbarkeit und guter Standort, die zentralen Qualitätskriterien vieler Kunden, erfüllt wurden. Aus der Kombination beider Faktoren wurden Marktanteilsgewinne aufgrund der verbesserten Kosten- und Qualitätsposition erwartet, über die sich die teilweise erheblichen Investitionen in ATMs amortisieren sollten. Diese Erwartung hat sich nach flächendeckender Einführung der beiden führenden Netze von ATMs, Bay Banks, Boston und Citibank, New York City bestätigt. ${ }^{3}$ Der beträchtliche Erfolg der ATMs im Hinblick auf die Position schlug jedoch unmittelbar in Stabilitätsprobleme um.

Ein erfolgreiches Commitment zieht den Versuch der Imitation nach sich. Dies kann als Gefangenendilemma dargestellt werden. ${ }^{4}$ Der betrachtete Markt war von Commitments in starre Faktoren bei Überkapazität, also hoher Wettbewerbsintensität, gekennzeichnet. Eine der wenigen Möglichkeiten, Marktanteile zu gewinnen, war die Einführung von ATMs. In Abbildung C.2.6 stehen sich die Wettbewerber, Citibank und die Konkurrenten gegenüber, in der Ausgangssituation je ohne ATMs. Beide Seiten können in ATMs investieren. Daraus ergeben sich die in den einzelnen Feldern angegeben Ergebniswirkungen, unten links die für Citibank, oben rechts diejenigen der Konkurrenten. Der gewonnene Positionsvorteil wird als "Zugewinn" notiert, dem Konkurrenten entsteht ein "Verlust".

1 Z.B. Einzahlungen, Scheckeinreichungen, Auszahlungen, Transfers jeder Art, das Ausstellen von Bankschecks, Ratenzahlungen etc.

2 Vgl. Zimmer, L.F., Reviving ATMs, in: Bank Management, Vol. 66, ISS. 6, June 1990, S. 58.

3 Vgl. Korzeniowski, P., Bank Reaps Return on Net Investment, in: Network World, Vol. 4, No. 33, August 17, 1987, S. 1; Brown, B., Bank Hones Its Strategic Edge, in: Network World, Vol. 5, No. 5, February 1, 1988, S. 1; Jaben, J., ATM Results, a.a.O., S. 38.

4 Zum Gefangenendilemma vgl. z.B. Luce, R.D., Raiffa, H., Games and Decisions, a.a.O., S. 95 ff. 


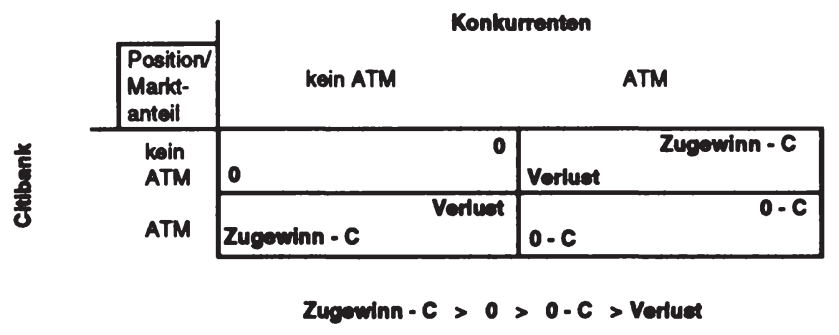

Abb. C.2.6: Imitation von ATM als Gefangenendilemma

ATMs sind starre Faktoren. Die Einrichtung eines ATM-Netzes ist ein Commitment. Der Barwert des Kostenvorlaufs aus dem Commitment sei mit $C$ angegeben. Citibank (wie auch BayBanks) investierte in ATMs, begab sich vom nordwestlichen Feld in das südwestliche. Marktanteilsgewinne, abzüglich C, waren die Folge, während die Wettbewerber Marktanteile verloren. In dem interdependenten Markt richteten jedoch schon nach kurzer Zeit die Wettbewerber ihrerseits ATMs ein. Schließlich stehen sich beide Seiten mit 0 - C gegenüber, d.h. ohne Marktanteilsgewinne, aber mit einem zu finanzierenden Kostenvorlauf. In der Innenstadt von Boston gibt es keine Geschäftsstraße, in der nicht alle $100-200 \mathrm{~m}$ ein ATM aufgestellt ist. "Many bankers are wondering if the millions of dollars invested in installing automated teller machines (ATM) have been worth it."1 Die Zweifel bestehen zu Recht.

Die Positionsgewinne durch Geschäftsprozeßintegration mittels ATMs waren nicht stabilisierbar. In anderen Fallstudien der Geschäftsprozeßintegration wurde herausgearbeitet, daß Stabilität durchaus möglich ist, wenn diese anderweitig z.B. auf der Marktführerschaft aufgebaut und durch KIS lediglich verstärkt wird. Auch diese Strategie scheiterte bei ATMs. Die Wettbewerber des Marktführers können dessen Anteilsvorteil durch eine Kooperationsstrategie neutralisieren wie im New Yorker Beispiel. Citibank als Marktführer war wohl als einziger überhaupt in der Lage, allein ein flächendeckendes ATM-Netz einzurichten. Im ansonsten fragmentierten Markt gab es keinen Wettbewerber, der ein ähnliches Commitment eingehen konnte. Daher gründete eine Gruppe von 8 New Yorker Banken das

1 Jaben, J., ATM Results, a.a.O.,S. 36. 
KIS "New York Cash Exchange" (NYCE) als Antwort auf die Einführung der ATMs durch Citibank.' Sie teilten sich ein Netz und benutzen es gemeinsam - eine horizontale Verflechtung gleicher Standorte. Dabei nimmt das Verflechtungspotential mit der Anzahl der Banken am Platz zu, gleichzeitig dividiert sich $\mathbf{C}$ durch die Zahl der Teilnehmer. Mit einer Kooperationsstrategie wird allerdings die Möglichkeit aufgegeben, das eigene ATM-Netz gegenüber den Wettbewerbern zur Differenzierung einzusetzen. Diese Strategie verfolgten Citibank und BayBanks, indem sie laufend die funktionalen Möglichkeiten ihrer ATMs erweiterten. So hat Citibank viele "ATMInteressierte" als Kunden behalten und einen "First-Mover-Effekt" realisiert. ${ }^{2}$

Ein weiteres Beispiel für die eigentliche Quelle von Stabilität bei Geschäftsprozeßintegration ist das Aufzuggeschäft. Otis Elevator stellt Aufzüge her und bietet Kundendienst für diese. Das Marktvolumen für Aufzugkundendienst in den USA war 1984 mit \$ 2 Mrd. doppelt so hoch wie das des Neugeschäfts. ${ }^{3}$ Der Otis-Kundendienst kann in (präventive) Wartung und Reparatur unterschieden werden. Das Abwicklungs- und Informationssystem Otisline unterstützte beide Aktivitäten in vieler Hinsicht. Täglich wurden durchschnittlich 4.300 telefonische Reparaturaufträge vom System entgegengenommen. Nach dem Kundenanruf wurde der räumlich nächste Monteur identifiziert und der Auftrag in seine Route einbezogen. Nach Erledigung der Reparatur wurden die Einzelheiten gespeichert, um aus der "Geschichte" des einzelnen Aufzugs sowohl für zukünftige Wartungs- und Reparaturmaßnahmen als auch für das Produktdesign zu lernen. Darüber hinaus war Otis durch Otisline direkt mit zahlreichen Aufzügen verknüpft. Täglich wurden die Bewegungsdaten und -profile automatisch übermittelt, aus denen präventive Wartungsmaßnahmen abgeleitet und terminiert wurden.

Nach Einführung von Otisline erreichte Otis erhebliche Positionsvorteile, besonders durch höheren Servicegrad und -qualität. Die Technologie von Otisline wäre von den Wettbewerbern, regionalen Kundendienstunter-

1 Vgl. Wiseman, Ch., Attack \& Counterattack: The New Game in Information Technology, in: Planning Review, Vol. 16, No. 5, September/October 1988, S. 6 ff.

$280 \%$ der Citi-Kunden nutzen ATMs regelmäBig für den überwiegenden Teil ihrer Banktransaktionen. Vgl. Jaben, J., ATM Results, a.a.O., S. 38.

3 Die Zahlen stammen aus dem Jahre 1984. Vgl. Stoddard, D., McFarlan, F.W., Otisline, Boston, Massachusetts, Harvard Business School Case No. 9-186-304, 1986, S. 1 ff. 
nehmen einerseits und USA-weiten Unternehmen mit weit geringeren Marktanteilen andererseits, prinzipiell leicht kopierbar gewesen. Otis aber war der einzige Anbieter, der regionale Skaleneffekte und USA-weite Skaleneffekte kombinieren konnte. Regionale Skaleneffekte waren, etwa im Vergleich zu Westinghouse, notwendig, um eine eigene Kundendienstorganisation vorzuhalten, die Voraussetzung für kurze Wartezeiten bzw. einen hohen Servicegrad. Gegenüber den regionalen Wettbewerbern konnte Otis durch den USA-weiten Skaleneffekt das Commitment in Otisline auf ein hohes Volumen verteilen. Die eigentliche Quelle der Stabilität der Position von Otis ergibt sich also aus der Marktführerschaft. Otisline schafft das passende "Leverage" jener Stabilität.

Geschäftsprozeßintegration hat eine erhebliche Wirkung auf die Wettbewerbsposition der Unternehmung. Die vertikale Verknüpfung des Geschäftsprozesses zu einem störungsarmen, schnittstellenfreien Ablauf kann zu einer völligen Neudefinition des Geschäfts führen, oder die Art des Geschäfts wesentlich verändern. In allen analysierten Beispielen ergaben sich jedoch erhebliche Stabilitätsprobleme. Ein interessanter Nebeneffekt der Geschäftsprozeßintegration ist es, daß sie neben der schnittstellenfreien Verknüpfung der Geschäftsaktivitäten auch die horizontale Verflechtung ermöglicht. So war bei Batterymarch der Prozeß der Portfoliooptimierung in allen Depots im wesentlichen gleichartig.

Abschließend sei ein weiteres Beispiel skizziert, in dem die Bildung vertikaler Verknüpfungen gleichzeitig das Ausschöpfen horizontaler Verflechtungen ermöglicht: das Konzept der Informationsraffinerie. Die Informationsraffinerie nimmt ein undifferenziertes, großes Volumen von Rohdaten auf, konvertiert diese in elektronische Form, extrahiert inhaltliche Einheiten und rekombiniert diese Einheiten in neuer Weise. Der Output kann verkauft werden als Endprodukt, als Rohdaten oder als Input einer nachgelagerten Informationsraffinerie. ${ }^{1}$ In Abb. C.2.7 wird die bislang als Verlag zu bezeichnende MCGraw Hill, Inc. als Informations raffinerie interpretiert. ${ }^{2}$

Daten und Informationen werden auf verschiedenste Arten generiert. Sie werden in den einzelnen Gesellschaften inhaltlich aufbereitet und in elek-

1 Vgl. Clippinger, J.H., Konsynski, B.R., Information Refineries, a.a.O., S. 73 ff.

2 Zum Geschäftsprozeß von McGraw Hill vgl. McGraw-Hill, Product Support Architecture, New York, New York, 1985, S. 4. 
tronischen "Masterfiles" abgelegt. Von dort aus gehen sie in eine (mehrere) Produkt-Plattform(en) ein, auf deren Basis eine Fülle von Produkten entsteht, die als Printmedien oder elektronische Medien produziert und vermarktet werden. Durchgängige elektronische vertikale Verknüpfungen sind auch hier der Hauptansatzpunkt der Geschäftsprozeßintegration von der Datengenerierung bis zur Distribution.

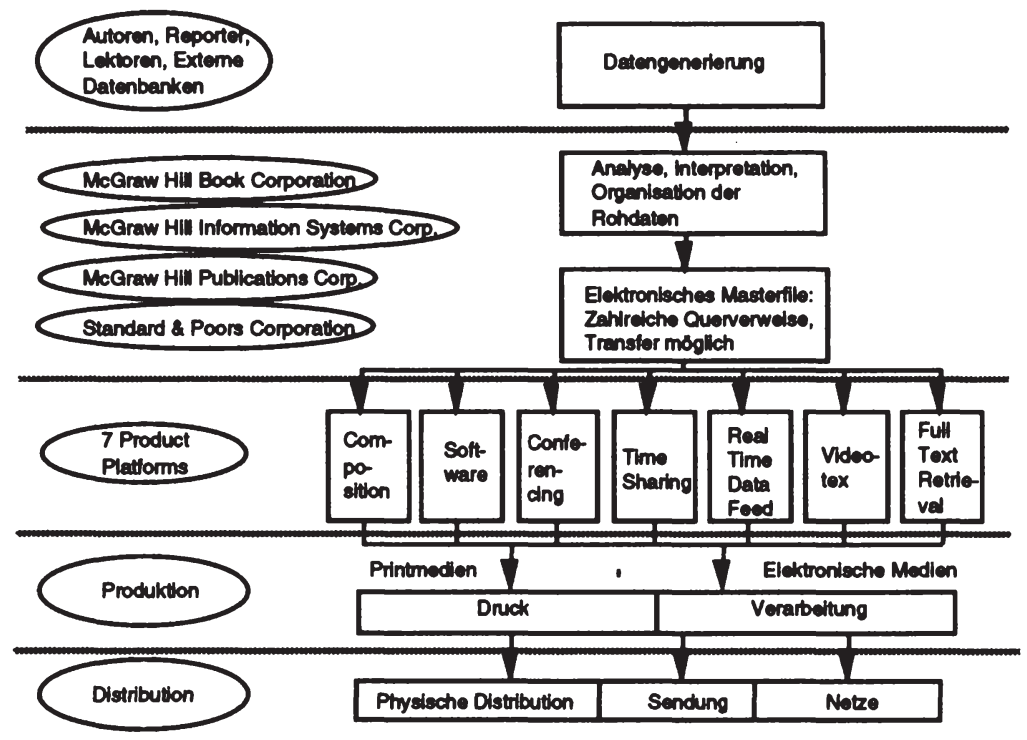

Abb. C.2.7: McGraw-Hill, Inc. als Informationsraffinierie

Als Informationsraffinerie wird sich McGrawHill aber erst bezeichnen lassen, wenn ein wirkliches "Cracken"1 von Information erfolgt. Dazu ist die Verflechtung auf den einzelnen Stufen, d.h. z.B. die horizontale Verflechtung der Masterfiles oder der Produktplattformen, von entscheidender Bedeutung. Suchkriterium für ein solches synergetisches Verflechten sind Gleichartigkeiten. Dies bedeutet auf der Inputseite die Mehrfachverwendung gleicher Dateninputs, auf der Outputseite die Wertsteigerung durch intelligentes Verflechten von Information.

1 Den Begriff der Informationsraffinierie entlehnen Clippinger und Konsynski aus dem Vergleich am Cracken von Erdöl. Vgl. Clippinger, J.H., Konsynski, B.R., Information Refineries, a.a.O., S. 74. 


\subsection{Geschäftsnetzwerkintegration}

Mit der KIS-gestützten Geschäftsnetzwerkintegration werden elektronische integrative Verbindungen über die (kontraktuellen) Grenzen der Unternehmung oder gar des Geschäftssystems hinausgezogen. Bereits 1966 berichtete Kaufmann von "Data Systems That Cross Company Boundaries"1, dem zahlreiche Arbeiten zur Geschäftsnetzwerkintegration unter den Stichworten Inter-Organisational Systems, Electronic Data Interchange und Electronic Integration folgten. ${ }^{2}$ Die Geschäftsnetzwerkintegration ist die in der Literatur mit Abstand am breitesten diskutierte Form der strategischen Integration mit KIS. ${ }^{3}$ Neben der hohen Auffälligkeit durch die Beteiligung mehrerer Parteien an einem Netz mag dies darauf zurückzuführen sein, $\mathrm{da}$ eine technische und teilweise organisationale Integration kontraktuell nicht integrierter Unternehmungen erfolgt. Diese aus klassischer Perspektive kontraintuitive Beobachtung, in dieser Arbeit als Substitutionalität der Integrationsinstrumente verallgemeinert, bedeutet die Bildung zuvor nicht möglicher Verbindungen und mag die Ableitung von Erkenntnissen für neue Organisationsformen erlauben.

1 Kaufmann, F., Data Systems That Cross Company Boundaries, in: Harvard Business Review, Vol. 44, January-February 1966, S. 141.

2 In der jüngeren Literatur wird zwischen Electronic Integration, Electronic Data Interchange (EDI) und Interorganisational Systems (IOS) unterschieden. Elektronische integrative Verbindungen entsprechen dem Konzept der Electronic Integration, während EDI und IOS eher auf den Technologieaspekt abstellen. Vgl. Venkatraman, N., Kambil, A., The Check's Not in the Mail: Strategies for Electronic Integration Tax Return Filing, Sloan Managment Review, Vol. 32, Winter 1991, S. 34 und dort angegebene Literatur.

3 Eine Auswahl sind Johnston, H.R., Lawrence, P.R., Vertical Integration, a.a.O.; Konsynski, B.R., McFarian, F.W., Information Partnerships, a.a.O., S. 114 - 120; Blackburn, J.D., The Quick Response Movement in the Apparel Industry: A Case Study in Time Compressing Supply Chains, in: Time-Based Competition, Homewood, Irwin, Illinois, 1991, 246 - 272; Benjamin, R.I., DeLong, D.W., Scott Morton, M.S., The Realities of Electronic Data Interchange: How Much Competitive Advantage?, Working Paper No. 166, Massachusetts Institute of Technology, Center for Information Systems Research, Cambridge, Massachusetts, 1988; Copeland, C.G., McKenney, J.L., Airline Reservation, a.a.O., S. 353 - 370; Barrett, S., Konsynski, B., Inter-Organization Information, a.a.O., S. 93 - 105; Venkatraman, N., Zaheer, A., Electronic Integration, a.a.O., 1989; Kolodziej, S., Success, a.a.O., S. 26 - 30; Venkatraman, N., Kambil, A., Electronic Integration, a.a.O., S. 34 - 43; McFarlan, F.W., Information Technology, a.a.O., S. 98 - 103; Cash, J.I., Konsynski, B.R., Competitive Boundaries, a.a.O., S. 134 - 142; Venkatraman, N., Short, J.E., Electronic Integration, a.a.O., und die dort jeweils angegebene Literatur sowie die Literatur zu Netzwerken und Quasi-Integration, z.B. Malone, T., Yates, J, Benjamin, R. I. Electronic Markets, a.a.O., S. 484 - 497; Jarillo, J.C., Ricard, J.E. Sustaining Networks, in: Interfaces, Vol. 17, No. 5, 1987, S. 82 - 91; Blois, K.J., Vertical Quasi-Integration, a.a.O., S. $253-272$. 
Drei sehr verschiedene Arten der Geschäftsnetzwerkintegration wurden identifiziert: die vertikalen Netzwerke, die Information Partnerships und die elektronischen Märkte. Sie unterscheiden sich in allen Strukturdimensionen des in dieser Arbeit vorgelegten Bezugsrahmens, insbesondere auch in ihren Wirkungen auf die Wettbewerbsposition und deren Stabilität. Unter den Fallstudien zur Geschäftsnetzwerkintegration bilden die vertikalen Netzwerke den Schwerpunkt. Sie sind vor allem dadurch gekennzeichnet, daß eine "focal Firm" als Träger eines elektronischen Netzes im Mittelpunkt der technischen Integration verschiedener, kontraktuell unabhängiger, in einem Geschäftssystem hintereinandergelagerter Unternehmungen steht. Die im folgenden Abschnitt vorgelegte Kurzfallstudie ist ein vertikales Netzwerk, in der die American Hospital Supply Corporation (AHSC) als "focal firm" das KIS trägt.

\subsubsection{Geschäftsnetzwerkintegration bei AHSC/Baxter}

American Hospital Supply Corporation (AHSC) war 1984 mit $25-30 \%$ Marktführer im Markt für Krankenhausbedarf. AHSC erzielte einen Umsatz von \$ 3,5 Mrd. und ein Jahresergebnis von \$ 238 Mio. aus drei Sektoren: Krankenhaus- und Laborbedarf, Spezialartikel für Ärzte und internationales Geschäft. Allein beim Krankenhaus- und Laborbedarf wurde ein Umsatz von \$2,9 Mrd. erzielt mit einer, von Pharmazeutika und größeren Anlagen abgesehen, umfassenden Produktpalette, u.a. IV-Lösungen, Krankenhausbekleidung und -material, Patientenpflegematerial, Instrumente sowie chemische und biomedizinische Produkte. AHSC belieferte nahezu alle 7.500 Krankenhäuser in den USA mit 135.000 verschiedenen Produkten. 1922 als reines Handelsunternehmen gegründet, nahm AHSC in den Nachkriegsjahren auch die Eigenproduktion auf. $46 \%$ des Umsatzes und 83 $\%$ des Gewinns wird den eigenproduzierten Artikeln zugerechnet. ${ }^{1}$ Auf der Ebene des Großhandels stand AHSC vielen hundert regional agierenden Wettbewerbern gegenüber. Auf Herstellerebene waren, neben kleinen Spezialfirmen, Johnson \& Johnson (O \& J), Abbott Laboratories und Baxter

1 Alle Zahlen für 1984, dem Ausgangsjahr der Kurzfallstudie. Vgl. Konsynski, B., Vitale, M.R., Baxter Healthcare Corporation: ASAP Express, Boston, Massachusetts, Harvard Business School, Case No. 9-188-080, revised July 9, 1990, S. 2 f.; Vitale, M.R., American Hospital Supply Corp.: The ASAP System (A) \& (B), Boston, Massachusetts, Harvard Business School, Cases No 9-186-005 und 9-186-006, 1986, S. 1 f. 
Travenol Corp. die Hauptwettbewerber. Das Produktprogramm überschnitt sich vor allem mit Abbott, insbesondere im für AHSC bedeutenden Markt für intravenöse Lösungen. Auf diese Marktstruktur wird zurückzukommen sein. Zunächst sei der Integrationsbereich skizziert.

\subsubsection{Einsatz von KIS im Geschäftsnetzwerk Krankenhausbedarf}

Abbildung C.2.8 enthält ein grobes Mapping der Distributionsseite des Geschäftssystems im Krankenhausbedarf von der Krankenhausabteilung bis zur Produktion. Dargestellt wird ein vereinfachter Auftragszyklus, bei dem zwei Krankenhausabteilungen bestellen, eine Order aus den jeweiligen Lagerbeständen eines Distributionszentrums, des AHSC Zentrallagers und des Lagers eines Lieferanten zusammengestellt wird, die dann teilweise postalisch und teilweise direkt geliefert wird. ${ }^{1}$ Außendienstmitarbeiter waren für die Bestellaufnahme und Marktbearbeitung in den ihnen zugewiesenen Krankenhäusern zuständig, wobei trotz teilweise zentralisiertem Einkauf das "Buying Center" die Fachabteilung war. Ware konnte, sofern sie in den Distributionszentren gelagert wurde, sofort von diesen angewiesen werden, ansonsten wurde sie erst hergestellt oder fremd bezogen. Im Distributionszentrum wurden dann krankenhausspezifisch Aufträge zusammengestellt und geliefert. Die Lieferzeit setzte sich zusammen aus Postlaufzeiten, Wartezeiten, Produktions- und Bearbeitungszeiten und Transportzeiten. In allen Teilen des Geschäftssystems entfiel ein Großteil der Aufgaben auf die Verwaltung von Bestell- und Lagerbeständen, auf Nachabstimmung und Statusanfragen. Ein 800-Betten Krankenhaus verursachte 50.000 Bestellungen pro Jahr bei Kosten von $\$ 25$ - 30 pro Bestellung und lagerte 30.000 verschiedene Artikel. ${ }^{2}$

1 Dieser Auftragszyklus ist zwar willkürlich gewählt, aber nicht untypisch. In realen Prozessen ist die Anzahl der Abteilungen höher und eine Fülle von Lieferanten zu beachten; auch muß nicht jeder Auftrag gebündelt werden. In einer Analyse realer Prozesse wäre die Relevanz der einzelnen Ströme modellhaft zu erfassen und alternativ mögliche Integrationsstrategien anhand des/der Modelle zu testen. Zur Entwicklung des Arguments ist hier das einfache graphische Modell jedoch hinreichend.

2 Vgl. Vitale, M.R., American Hospital Supply Corp., a.a.O., S. 1 - 3 

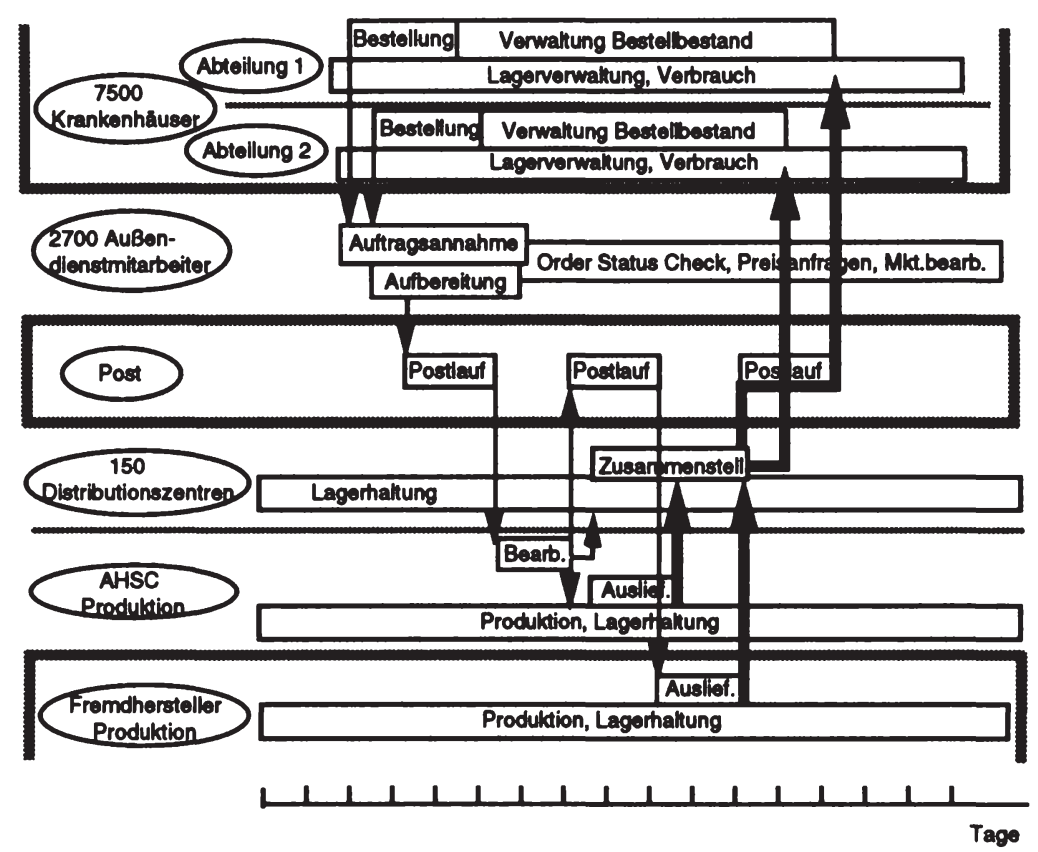

Abb. C.2.8: Integrationsbereich Distribution boi American Hoepltal Supply Corporation

AHSC begann angesichts jenes Volumens von Geschäftsaktivitäten schon Mitte der sechziger Jahre, erste Abwicklungssysteme einzusetzen. Das erste auf Lochkarten basierende KIS zwischen einigen Krankenhäusern und AHSC, Tel-American, wurde 1963 eingeführt.1 1967 folgte das "Analytic Systems Automatic Purchasing" (ASAP). ASAP 1 (ab 1967) nutzte weiterhin Lochkarten. Dem Krankenhaus wurde ein "Prime Vendor"-Status bei AHSC angeboten. Als Gegenleistung wurden die Kundenkosten des Bestellens und der Lagerhaltung wesentlich reduziert. Der im Krankenhaus aufgestellte Lochkartenleser bot zudem die Möglichkeit, Bestellmengen manuell telefonisch einzugeben anstatt eine Karte zu lochen. Mit ASAP 2 (ab 1972) wurde zusätzlich das sofortige Ausdrucken einer Bestellung und die elektronische Nachrichtenübermittlung zwischen AHSC, den Außen-

1 Eine detaillierte Beschreibung dieses und der folgenden Systeme findet sich bei Venkatraman, N., Short, J.E., Electronic Integration, a.a.O., S.6 - 25; Vitale, M.R., American Hospital Supply Corp., a.a.O., S. 3 - 5; Konsynski, B., Vitale, M.R., Baxter, a.a.O., S. 4 - 6 . 
dienstmitarbeitern und den Krankenhäusern möglich. ASAP 3 (ab 1980) erlaubte dem Krankenhaus die Eingabe der Orders auf Basis eines eigenen Nummernsystems, außerdem die Einrichtung elektronischer Ordner im Zentralrechner von AHSC, in denen die Bestellbestände jederzeit geprüft werden konnten. ${ }^{1}$ ASAP 4 (ab 1983) ermöglichte die technische Integration der Mainframes der Krankenhäuser mit denjenigen von AHSC. Alle manuellen Schritte des Kauf- und Abwicklungsprozesses wurden eliminiert, ausgenommen die Zustimmung zur Bestellung. Das Kundensystem produzierte eine Vorschlags-Bestelliste, die ein Disponent nur noch prüfte und sofort in den AHSC-Rechner einging.
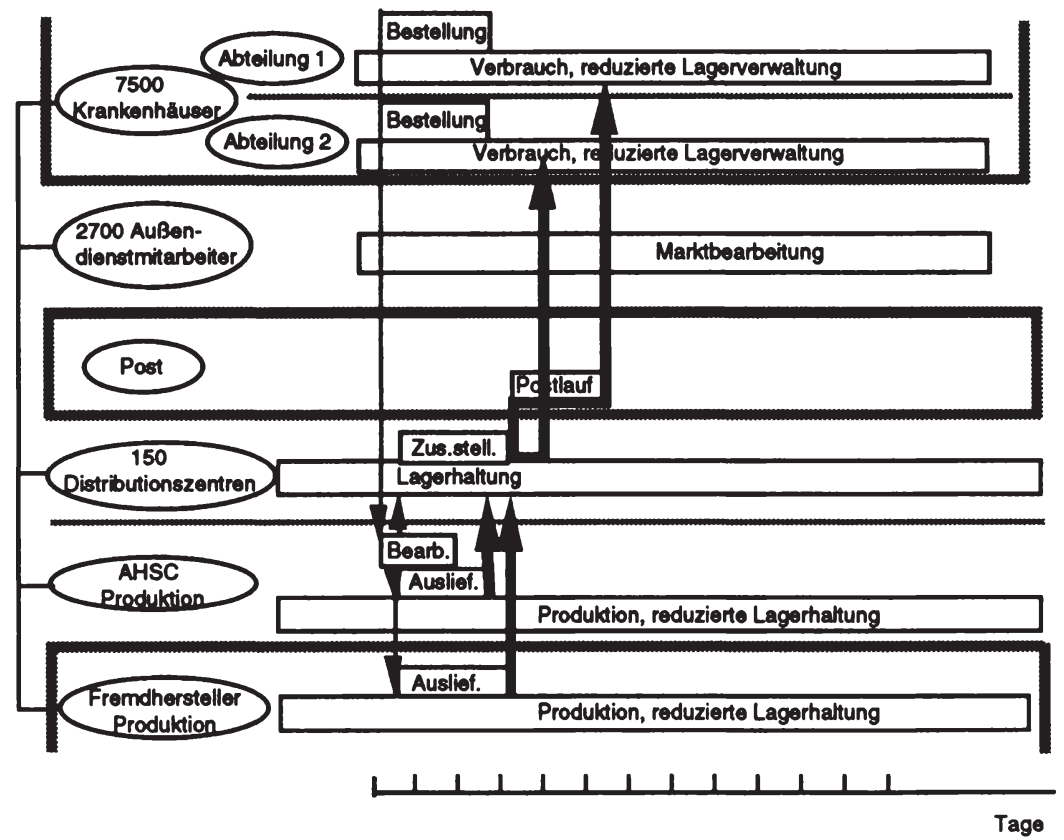

Abb. C.2.9: Distribution bei American Hospital Supply Corporation bis incl. ASAP 4

Mit ASAP 5 (ab 1985) erhielten die Krankenhäuser Personal Computer, so daß zahlreiche Funktionen nicht mehr Online ausgeführt werden mußten, wodurch die Telefonleitungskosten gesenkt werden konnten. Gleichzeitig hat AHSC ein "Reverse-ASAP" mit vielen Lieferanten eingeführt.

1 Vgl. o.V., Ordering System Dries Up Flow of Paperwork, in: Modern Healthcare, May 1982, S. 147. 
Entstehender Bedarf wurde mit den ASAP-Systemen ohne Verzögerung an den Lieferanten übermittelt, AHSC gab selbst Bestellungen nicht mehr auf, sondern der Lieferant ist für die jederzeitige Bedarfsdeckung zuständig, zudem wurden Informationen z.B. über Lagerbestände und Preiseausgetauscht. In Abb. C.2.9 sind die Veränderungen des Auftragszyklus bei AHSC für einen mit Abb. C.2.8 identischen Auftragszyklus skizziert.

ASAP führte zu einer sehr engen technischen Integration zwischen AHSC und den Krankenhäusern. AHSC erlangte dabei wesentliche Positionsvorteile in Bezug auf Zeit, Kosten und Qualität. Neben dem evidenten Zeitvorteil ${ }^{1}$ durch Beschleunigung des Auftragszyklus konnten die Kosten des Auftragsprozesses bei AHSC wesentlich reduziert werden. Eine (nominale) Verdreifachung des Umsatzes von 1975 bis 1984 wurde mit einer konstanten Anzahl von Außendienstmitarbeitern erreicht, Schwund und Abstimmungsverluste wurden reduziert, Lagerbestände gesenkt und ein Großteil der Verwaltungsarbeiten eliminiert. Dies gilt auch für das Krankenhaus. Die Beschleunigung des Auftragszyklus zog niedrigere Lagerbestände, sinkende Kosten pro Bestellung durch weniger Verwaltungsarbeiten und nachfragen, bessere Kontrolle von Lagerschwund sowie geringere Abstimmungsprobleme innerhalb der Krankenhauses nach sich. Damit verbesserte sich die Qualitätsposition durch die Senkung von Kundenkosten. Zusätzlich wirkte ASAP auf Signalkriterien der Qualität. Durch jederzeitige Einsehbarkeit in den (wirklichen) aktuelle Status der Lieferung wuchs das Vertrauen in die Pünktlichkeit der Lieferung - ein wichtiges Signalkriterium für den wahrgenommenen Servicegrad. ${ }^{2}$

Die Beobachtung von Positionsgewinnen läßt allerdings den Verdacht entstehen, daß Dauerhaftigkeitsprobleme bestehen. Dies erwies sich zumindest in fast jedem Fall der Geschäftsprozeßintegration. Die Geschäftsnetzwerkintegration weist jedoch einen entscheidenden Unterschied auf. Durch den

1 Im Jahr 1985 konnten $70 \%$ aller Bestellungen in 24 Stunden geliefert werden. Vgl. Spragins, E.E., Aikman, R., American Hospital Supply: Snaring New Business with Freebies and Bonuses, in: Business Week, April 8, 1985, S. 88. Nach dem Mapping erscheint dies lediglich bei Direktlieferung aus dem Lagerbestand der Distributionszentren möglich.

2 Vgl. Dhebar, A.S., American Hospital Supply Corporation: The ASAP System, Boston, Massachusetts, Harvard Business School Teaching Note No. 9-187-014, Rev. 02/88, 1988, S. 4. 
beschränkten Zugang zum Kunden können strukturelle Mobilitätsbarrieren gegenüber den Wettbewerbern aufgebaut werden. Diese sind im Gegensatz zu endogenen Mobilitätsbarrieren nicht ohne weiteres kopierbar. ${ }^{1}$ AHSC hat durch ein Bündel von technischer und kontraktueller Integration quasi strukturelle Mobilitätsbarrieren erreicht. Mit der Verknüpfung des AHSC-Zentralrechners und der Mainframes der Krankenhäuser ging die vollständige Abwicklung der Materialwirtschaft und Disposition der Krankenhäuser auf ASAP 4 über. Diese Maßnahme, die Kundenkosten beträchtlich senkte, setzte eine kontraktuelle Integration voraus, und zwar den Prime-Vendor-Status bei AHSC. In den technisch und kontraktuell vertikal verknüpften Krankenhäusern erreichte AHSC damit überwiegend mehr als die Hälfte des Bestellvolumens. Günstig wirkte sich dabei aus, daß AHSC über ein sehr breites Sortiment verfügte. Diese endogene Mobilitätsbarriere wurde mit der strukturellen Mobilitätsbarriere des Zugangsvorteils zum Kunden kombiniert, oder umgekehrt, durch das breite Sortiment wurde der Zugangsvorteil erst möglich.

Zur Imitation dieser Integrationsstrategie kamen die direkten Wettbewerber des Großhandels nicht in Betracht, da sie weder über eine hinreichende Sortimentsbreite noch über ein Volumen verfügten, das ein solches System rechtfertigte. Allerdings war es den USA-weiten Hersteller-Konkurrenten durchaus möglich, Teile der Integrationsstrategie zu imitieren. Auch sie begannen zehn Jahre nach ASAP 1 Abwicklungssysteme zu den Krankenhäusern einzurichten. ${ }^{2}$ Diese hatten jedoch gegenüber ASAP einen entscheidenden Unterschied: Es handelte sich um kooperative Systeme, ${ }^{3}$ die auch die Bestellung von Produkten anderer Hersteller erlaubten: J\&J's Cooperative Action Plus (COACT) sowie Abbott's Quiklink.

Angesichts der Reaktionsgeschwindigkeit der Imitation bei Bank-ATMs mag es verwundern, warum Imitationsinitiativen im Krankenhausbedarf lange Zeit unterblieben. Dies ist damit zu erklären, daß sowohl J\&J als auch Abbott auf Herstellerebene mit AHSC kaum konkurrierten, AHSC jedoch, insbesondere für J\&J, einer der wichtigsten Händler war. In ASAP sah J\&J daher zunächst lediglich ein Mittel, mit dem einer ihrer Händler, AHSC, die Krankenhäuser als Endkunden besser $\mathrm{zu}$ versorgen strebte. 4

1 Vgl. Abschnitt B.1.3.2.1.

2 Vgl. Spragins, E.E., Aikman, R., American Hospital Supply, a.a.O., S. 88 und 89.

3 Vgl. Venkatraman, N., Short, J.E., Electronic Integration, a.a.O., S. 19.

4 Vgl. Venkatraman, N., Short, J.E., Electronic Integration, a.a.O., S. 15. 
In Abb. C.2.10 ist, mit der eingeführten Notation, d.h. starke Pfeile für Materialflüsse, einfache Linien für elektronische vertikale Verknüpfungen, das Geschäftssystem Krankenhausbedarf skizziert. Es stellt eine äußerst fragile Wettbewerbssituation dar. Der dominante Zugangskanal zu den Krankenhäusern ist ASAP mit aufkommenden Konkurrenzsystemen. Aus Sicht der Krankenhäuser ist dabei die Aufnahme von mehr als einem Bestellsystem keineswegs attraktiv. Der entscheidende Vorteil von ASAP, die Übernahme der gesamten Materialverwaltung, ist nur sinnvoll, wenn ein möglichst großes Bestellvolumen nur über ein System abgewickelt wird. Aus Sicht der Krankenhäuser besteht daher ein starker Druck auf die horizontale Verflechtung der Bestellsysteme zu einer gemeinsamen Plattform.

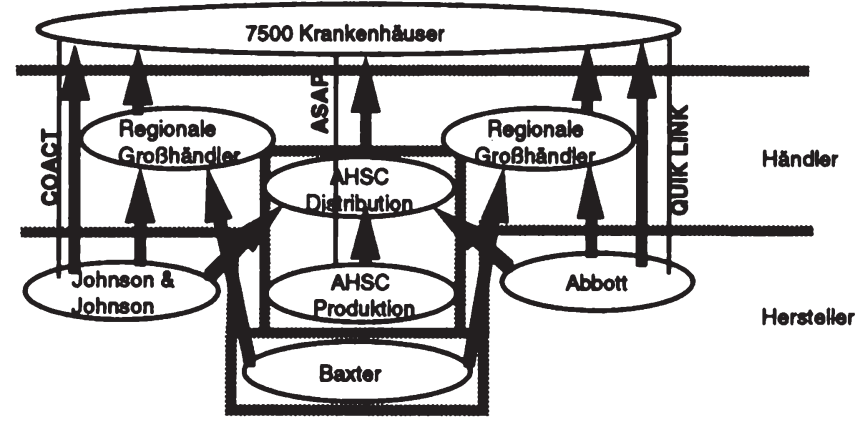

Abb. C.2.10: Geschăfssystem Krankenhausbedarf USA, Mărz 1985

Die zweite Quelle der Fragilität war der Hersteller Baxter. Mit einem Umsatz von \$1.8 Mrd. war Baxter ein bedeutender, aber spezialisierter Hersteller im Markt für Krankenhausbedarf. Baxter verfügte im Vergleich zu AHSC, J\&J und Abbott weder über ein hinreichend breites Sortiment noch über ein hinreichendes Volumen, um ein eigenes Abwicklungssystem zu den Krankenhäusern knüpfen zu können. Mit Einrichtung eines potentiellen, von AHSC, J\&J und Abbott kooperativ betriebenen Abwicklungssystems müßte Baxter um die Existenz seines Distributionskanals, die regionalen Händler, fürchten. Das System könnte Baxter den Zugang zu seinem Markt "abschneiden" oder behindern und damit eine unüberwindbare strukturelle Mobilitätsbarriere darstellen. Baxter mußte also reagieren. A m 20. Juni 1985 bot Baxter Travenol Laboratories den Aktionären der Ameri- 
can Hospital Supply Corporation einen "unerwarteten"1 hostile Takeover für \$ 3.7 Mrd. zur Bildung der Baxter Healthcare Corporation an. ${ }^{2}$

\subsubsection{Kontraktuelle Integration zur Baxter Healthcare Corporation und die Folgen}

Mit der kontraktuellen Integration von AHSC und Baxter in eine Firma veränderte sich die Situation erheblich. Der für ASAP zuständige Unternehmensteil von AHSC übernahm die Verantwortung für die organisationale und technische Integration des KIS der neuen Einheit,

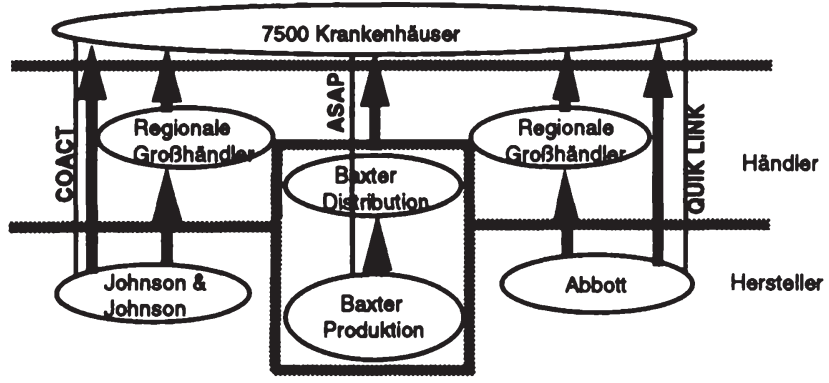

Abb. C.2.11: Geschăftssystem Krankenhausbedarf USA, Ende 1985

Baxter Healthcare. ${ }^{3}$ J\&J und Abbott verloren einen wesentlichen Absatzkanal an ihren Wettbewerber Baxter und fuhren den über ASAP gelieferten Abverkauf zurück.

Demgegenüber forcierten sie umgehend und erheblich ihre Bemühungen, COACT und QUIKLINK auszubauen und erreichten 1986 eine ähnliche

1 Konsynski, B., Vitale, M.R., Baxter, a.a.O., 1990, S.6. Die Überraschung wurde auch von der Finanzpresse geteilt, die 1985 insbesondere vermerkte, daB die kleinere Baxter Corp. die wesentlich größere AHSC übernimmt. Aus Sicht der dargestellten Zwangssituation Baxters erschien die Übernahme aber keineswegs überraschend.

2 Dem war der Versuch von AHSC vorausgegangen, die mit 400 Krankenhäusern größte Krankenhauskette der USA, die Hospital Corporation of America, zu übernehmen. Bei Fusion wäre den AHSC-Aktionären ein Wert von $\$ 36,50$ pro Aktie zugekommen. Das Angebot von Baxter entsprach demgegenüber $\$ 50$ pro Aktie. Dem höheren Angebot wurde der Vorzug gegeben. Baxters Senior Vice President Richard B. Egen gab ASAP als den wesentlichen Pluspunkt von AHSC bei der Übernahme an. Vgl. Konsynski, B., Vitale, M.R., Baxter, a.a.O., S. 6 - 7.

3 Über die Zusammenfassung des KIS bei Baxter Healthcare berichten Main, T.J., Short, J.E., Managing the Merger: Building Partnership Through IT Planning at the New Baxter, in: MIS Quarterly, Vol. 13, December 1989, S. 469 - 484. 
Funktionsfähigkeit wie ASAP. ${ }^{1}$ Dies entspricht der Reaktion der Konkurrenten von Citibank und Baybank bei der Einführung von Bank-ATMs. Aufgrund ihres im Vergleich zu Baxter Healthcare schmaleren Produktprogramms bezogen J\&J und Abbott weitere Sortimente von Fremdherstellern, und wandelten damit ihre Systeme zu kooperativen Systeme um. Ende 1988 boten sie auf ihren Systemen den Krankenhäusern die Produkte von über 30 Herstellern an. ${ }^{2}$ Baxter, nunmehr eine Unternehmung mit $\$ 5,8$ Mrd. Umsatz ${ }^{3}$, stand in vielen Märkten in scharfem Wettbewerb mit beiden. Ein Preiskrieg mit Abbott zwischen 1985 und 1986 im für Baxter sehr bedeutenden Markt für intravenöse Lösungen ließ die Profitabilität sinken. 1987 verlor Baxter zudem einen wichtigen Großkunden, die Voluntary Hospitals of America, an Abbott. 4

ASAP, vormals noch strukturelle Mobilitätsbarriere, wurde durch COACT und QUIKLINK neutralisiert. Dies wurde unterstützt durch eine "neue Regeln"-Strategie von J\&J sowie Abbott, die mit ihrem vormals bedeutendsten Händler in Konkurrenz traten und in zentralen Produktfeldern hoher wettbewerblicher Interdependenz Preiskämpfe austrugen. Baxter Healthcare strebte den Ausbau von ASAP an. Mit ASAP 8 wurde ab 1986 auch die Rechnungsstellung und die Zahlung auf das System übernommen. 1988 wurde mit ASAP Express erstmals auch von Baxter Healthcare ein kooperatives System angeboten, womit die Krankenhäuser auch Wettbewerbsprodukte bestellen konnten. Dabei hatte das System eine Priorität (Bias) für das Baxters-Angebot, d.h. es würde eigene Produkte zuerst benennen. Mit

1 Vgl. Konsynski, B., Vitale, M.R., Baxter, a.a.O., S. 7.

2 Vgl. Venkatraman, N., Short, J.E., Electronic Integration, a.a.O., S.19 f. Venkatraman und Short drehen die Abfolge der Vorgänge sogar geradezu um und begründen die Einführung von COACT und QUIKLINK mit der Ubernahme von AHSC durch Baxter. Dies deckt sich jedoch nicht mit dem tatsächlichen Ablauf. Die Bestellsyteme waren bereits vor dem Ende der Entwicklung eingeführt, als Baxter sich zur Übernahme entschloB. Vgl. Spragins, E.E., Aikman, R., American Hospital Supply, a.a.O., S. 89; Konsynski, B. R., McFarlan, F. W., Information Partnerships, a.a.O., S. 116. ASAP war, wie Senior Vice President von Baxter, Richard B. Egen, bestätigt, der Kern der Übernahmeentscheidung, nicht COACT und QUIKLINK die Folge der Übernahme. Vgl. Konsynski, B., Vitale, M.R., Baxter, a.a.O., S. 6 f. Andernfalls wäre die Logik der Übernahmeentscheidung, zumindest aus Sicht des in dieser Arbeit vorgelegten Ansatzes, nicht mehr gegeben und die Übernahme tatsächlich als "überraschend" einzustufen. Gleichwohl ist nicht mu die Übernahme von AHSC durch Baxter, sondem der erheblich beschleunigte Ausbaus von COACT und QUIKLINK mit der hier entwickelten Perspektive der Situation eines Gefangenendilemmas gut erklärbar.

3 In 1986. Der Umsatz 1989 betrug \$6,8 Mrd. Vgl. Baxter Healthcare Corporation, Annual Report to Shareholders, February 20, 1989, S. 20.

4 Vgl. Siler, F., Will Another Round of Surgery help Baxter?, in: Business Week, 30. April 1990, S. 92. 
ASAP Express Powerbase wurde das kooperative System 1990 ausgebaut und Just-in-Time-Prinzipien zur weiteren Lagerreduzierung eingeführt. COACT und QUIKLINK waren jedoch nach wie vor ebenbürtig. Baxter erreichte 1989 einen ROE von nur 11,1\%, Abbott von 33,1\% und Johnson \& Johnson von $28,3 \%$.

Wie bei der Geschäftsprozeßintegration z.B. mit Bank-ATMs entstand bei der strategischen Integration mit einem KIS-gestützten Geschäftsnetzwerk im Krankenhausbedarf ein Gefangenendilemma. Ein "First Mover" kann einen erfolgreichen "ersten Zug" für sich verbuchen, der jedoch durch Imitation oder neue Regeln neutralisierbar ist; es sei denn, es kann ein Dauerhaftigkeitsvorteil aufgebaut werden, der außerhalb der Integrationsstrategie begründet ist. Der Versuch von Baxter Healthcare, durch Hinzufügen weiterer Optionen zum ASAP einen für die Wettbewerbsposition relevanten Vorsprung vor Abbott und J\&J zu erzielen, ist an der Imitierbarkeit gescheitert. 1990 hat Baxter dies erkannt und begonnen, die Wettbewerbsposition und deren Dauerhaftigkeit auch jenseits der Integrationsstrategie auszubauen. Durch strategische Allianzen mit IBM, Kraft und Waste Management soll den Krankenhäusern ein umfassendes, KIS-gestütztes Krankenhausmanagementsystem angeboten werden, das auch den Nahrungsmittelbereich und die Abfallentsorgung mitumfaßt. ${ }^{1}$

Im Hinblick auf die Integrationsstrategie hatte Baxter kaum Alternativen. Insbesondere die Übernahme von AHSC schien geboten. Dies gilt auch für die Schaffung einer elektronischen vertikalen Verknüpfung an und für sich. Es ist das Kennzeichen des Gefangenendilemmas, daß die Einführung des Systems eine dominante Strategie gegenüber der Nichteinführung ist. D.h. die Einführung erbringt unabhängig von den Aktionen der anderen Spieler einen höheren Zielwert als die Nichteinführung - mit Ausnahme der vereinbarten Kooperation. Aus dieser Perspektive kann eine mögliche Alternative zum Vorgehen von Baxter abgeleitet werden. Dies wäre ein Kooperationsangebot an J\&J und Abbott gewesen, eine gemeinsame, möglicherweise auch anderen Herstellern zugängliche Plattform für ASAP zu schaffen. Das Potential für eine horizontale Verflechtung ist aus der Kundenperspektive erheblich. Könnte ein Krankenhaus sämtliche Beschaffungsvorgänge über ein einziges System abwickeln, würden ihm Doppelarbeiten erspart. Baxter hätte vermutlich einen akzeptablen Nutzungsbeitrag

1 Vgl. Siler, F., Baxter, a.a.O., S. 92. 
für die Bereitstellung des Systems realisieren können, während J\&J und Abbott sich den Aufbau eines zentralisierten eigenen Abwicklungsystems erspart hätten, das Kompatibilitätsprobleme zu ihrer dezentralen Führungsstruktur aufwies. ${ }^{1}$ Problematisch ist aus Kundenperspektive bei dieser Lösung die Wirkung auf den Wettbewerb in der Branche. ${ }^{2}$

\subsubsection{Vertikale Netzwerke}

Vertikale Netzwerke sind eine elektronische vertikale Verknüpfung eines Geschäftsnetzwerks, die von einer Unternehmung (focal Firm) getragen wird. Bekannte weitere Beispiele neben dem ASAP System von Baxter sind die Reservierungssysteme der Fluggesellschaften American Airlines und United Airlines sowie das Bestellsystem Economost des Pharmagroßhändlers McKesson. ${ }^{3}$ Einige andere sind das Abwicklungssystem EMCON des Luftfrachtversenders Emery, mit dem Kunden nicht nur ihre Luftfrachtversendung automatisieren, sondern auch jederzeit den Status der Sendung in Emerys Mainframe einsehen können, das KIS des Modeherstellers Benetton, über das eine weltweite Integration erfolgt, oder das "Electronic Retailing", in dessen Rahmen ganze Geschäftssysteme, z.B. von der Textilienherstellung bis zum Point-of-Sale, technisch integriert werden. ${ }^{4}$

In allen Fällen der elektronischen strategischen Integration durch vertikale Netzwerke zeichnet sich ein ähnliches Grundmuster ab. Die focal Firm im vertikalen Netzwerk trägt ein KIS. Seine vor- und/oder rückwärtsgerichteten elektronischen Verbindungen in das Geschäftsnetzwerk, d.h. mit den Zulieferern, Kunden oder Händlern, bilden das vertikale Netzwerk. Die elektronischen vertikalen Verknüpfungen verbinden Material- und Informationsflüsse zwischen den Netzwerkunternehmungen. Sie betreffen da-

1 Vgl. Vitale, M.R., American Hospital Supply Corp.: The ASAP System (A) \& (B), MIS Teaching Note, Boston, Massachusetts, Harvard Business School, Case No 5-188-119, revised April 1989, S. 7.

2 Der Entwurf eines branchenumfassenden Flugreservierungssystems wurde vom U.S. Justice Department im Jahre 1967 als Per-se-Verletzung des Wettbewerbsrechts angesehen. United States Civil Aeronautics Board, Report to Congress on Airline Computer Reservations Systems, 1983, Docket 41207.

3 Vgl. Venkatraman, N., Kambil, A., Strategies for, a.a.O., S. 33. Einen zusammenfassenden Überblick über die Reservierungssysteme der Fluggesellschaften geben Copeland, C.G., McKenney, J.L., Airline Reservation, a.a.O., S. 353 - 370; desgleichen für das Economost System McKessons Clemons, E.K., Row, M., McKesson, a.a.O., S. 36 - 50.

4 Vgl. 2.B. Blackburn, J.D., (Hrsg.), Time-Based Competition, a.a.O., S. 252 - 264; Vitale, M., Earl,R., British Home Supply (BhS) a.a.O. 
mit in erster Linie den Auftragsprozeß, d.h. die tatsächliche Leistungserstellung, z.B. die Abwicklung der Flugreservierung und -zahlung, die Bestellung und Lieferung von Pharmazeutika, die Abwicklung von Luftfrachtsendungen. Vertikale Netzwerke werden weniger für den Managementprozeß und nur vereinzelt für den Entwicklungsprozeß eingesetzt. ${ }^{1}$ Charakteristisch für sie sind hohe Datenvolumina und nur mäßige Unsicherheit, dabei ist die Bildung elektronischer Verknüpfungen oft kunden-, lieferanten-, räumlich-physisch- und personalspezifisch. Entstehen daraus einseitige Abhängigkeiten, sind Verteilungsprobleme innerhalb des Netzes die Folge: Ein vertikales Netzwerk wird häufig eine zusätzliche Rente (Positionsgewinne) produzieren, nur deren Verteilung ist fraglich. Schlimmstenfalls können spezifische Investitionen nicht nur die Abschöpfung von Quasi-Renten, d.h. die Abschöpfung der in das System selbst versunkenen Kosten, nach sich ziehen, sondern auch noch durch Imitation und Neue-Regeln-Strategien der Wettbewerber die Rentenerzielung im Grundgeschäft gefährden. So kann Baxters Entscheidung, AHSC zu übernehmen, um ASAP nutzen zu können, in dieser Hinsicht interpretiert werden. Letztlich hat Baxter eine höhere Wettbewerbsintensität für sich selbst erzeugt. Baxter mußte damit nicht lediglich die potentielle Rente der durch ASAP gewonnenen Positionsvorteile abgeben, sondern schmälerte durch Neue-Regeln-Strategien der Wettbewerber auch darüber hinaus sein Potential zur Erzielung von Renten.

\subsubsection{Vertikale Netzwerke und Wettbewerbsposition}

Daraus ist ein Zusammenhang zwischen der Stabilität der Wettbewerbsposition und der Verteilung der Rente bei vertikalen Netzwerken ableitbar. Die Verteilung einer Rente hängt davon $a b$, welche Abhängigkeitsbeziehungen im vertikalen Netzwerk bestehen zwischen den Eigentümern aufeinander angewiesener, spezifischer Faktoren. Eine steigende (horizontale) Wettbewerbsintensität wird im vertikalen Netzwerk die Position des Eigentümers im Bezug auf starre Faktoren verschlechtern. Bei Baxter bedeutete dies die Weitergabe von Rente in Form von Preiszugeständnissen und kostenloser Ausstattung mit ASAP-Terminals an die

1 Dies bedeutet freilich nicht, daß sich die betreffenden Unternehmungen nicht mit anderen Integrationsinstrumenten als den hier betrachteten volumenorientierten Kommunikationsund Informationssystemen abstimmen, etwa in der Produktentwicklung. 
Krankenhäuser. Instabile Wettbewerbspositionen verstärken die Abschöpfung von Renten im vertikalen System, erhöhen insofern die Verteilungsproblematik.

Damit erhebt sich die Frage der Stabilität und, wird nach möglichen Reaktionen der Unternehmung gefragt, auch der Flexibilität der Wettbewerbsposition bei vertikalen Netzen. Die Einrichtung einer elektronischen vertikalen Verknüpfung stellt ein Commitment in einen starren Faktor dar. Beispielsweise hat der Pharmahändler McKesson mit seinem System Economost ein durch Hand-Held-Computer zugängliches Bestellsystem eingerichtet, das technisch und ökonomisch auf die Zusammenarbeit mit kleinen, unabhängigen Drogerien ausgerichtet ist. McKesson bietet der Drogerie Just-in-Time-Lieferung bei einem $99 \%$-igen Servicegrad, was eine über die Regalfläche hinausgehende Lagerhaltung überflüssig macht, eine vollständige Automatisierung der Bestellungen erlaubt und ein vollständiges Drogerienmanagementsystem umfaßt. ${ }^{1}$ Das System würde unbrauchbar, wollte McKesson große Drogerieketten beliefern, die eigene Logistik, zentralisierte Beschaffung, eigene Managementsysteme und Bestellpolitik haben. Die Investition in das Economost-System ist (u.a.) in dieser Hinsicht starr auf das angestammte Geschäft mit unabhängigen Drogerien ausgerichtet. Die Starrheit begründet direkte Flexibilitätsprobleme.

1 Vgl. Clemons, E.K., Row, M., McKesson, a.a.O., S. 38 ff. 


\subsubsection{Stabilität vertikaler Netzwerke}

Beispiel eines vertikalen Netzes mit hoher Stabilität ist der global anbietende Modehersteller Benetton. In Abb. C.2.12 ist grob vereinfacht ein Saisonzyklus bei Benetton dargestellt. ${ }^{1}$ Benetton ist "focal Firm" eines interessant geknüpften vertikalen Netzwerks. Nur sehr wenige zentrale Geschäftsaktivitäten werden innerhalb der kontraktuellen Grenzen der Unternehmung ausgeführt, und zwar das Färben, die Logistik, teilweise das Design.

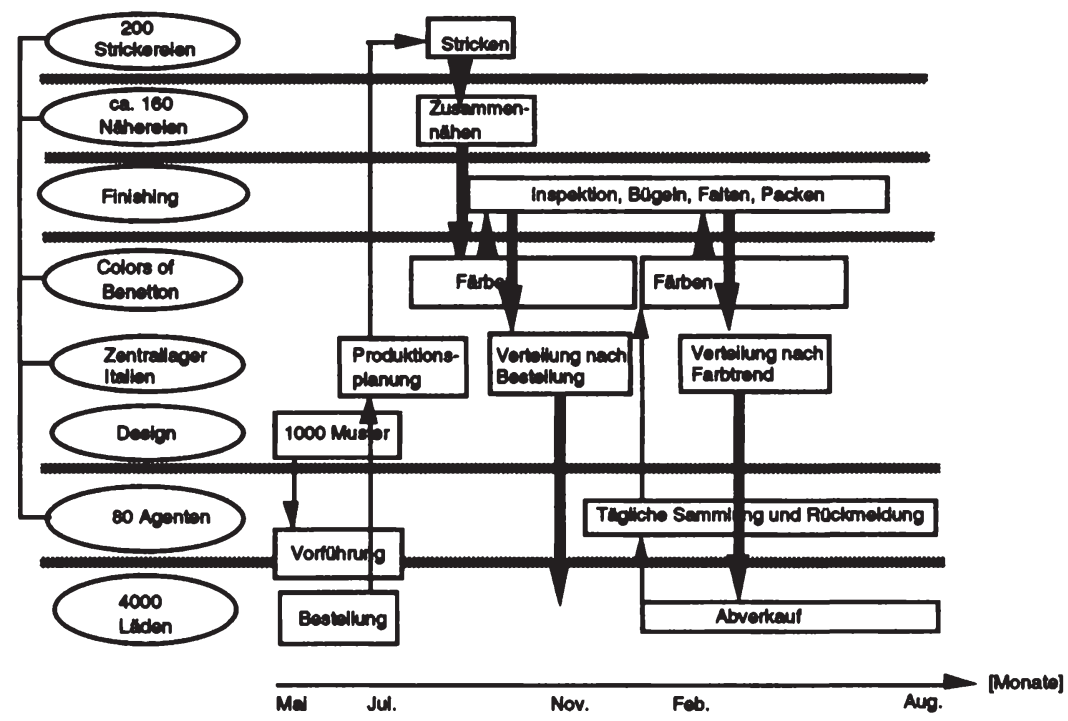

Abb. C.2.12: Bention S.p.A.: Saleonzyldus

Sowohl die Produktion als auch die Distribution übernehmen unabhängige Firmen. Dies ermöglicht Benetton niedrige Integrationskosten durch niedrige Agencykosten - die sehr kleinen, zuliefernden und abnehmenden Firmen werden jeweils vom Eigentümer geführt. ${ }^{2}$ Ein weltumspannendes

1 Vgl. Vitale, M., Benetton S.p.A., Industrial Fashion, Harvard Business School Case No. 9-188-003, Boston 1987, S. 1 - 9; Zottola, L., The United Systems of Benetton, 1990, S. 70.

2 Über ein anderes Beispiel aus der vertikalen Struktur der italienischen Textilindustrie (Fußnote wird auf der nächsten Seite fortgesetzt) 
KIS verbindet Benetton, einige Zulieferer und die ca. 70 Agenten, die jeweils ein Gebiet von Läden betreuen. Das System dient zum Austausch jeglicher Informationen, vor allem zur Sammlung der Bestellungen, dem Zahlungsverkehr und zur täglichen Erfassung von Abverkaufszahlen. Dabei sind die einzelnen Läden nicht technisch integriert. Dies bestätigt die Hypothese, daß der Einsatz der KIS wesentlich durch das Volumen bedingt ist. Bei Benetton endet hohes Volumen beim Agenten, auf Ladenebene ist das Volumen gering: Bei $\$ 1,2$ Mrd. Jahresumsatz auf Herstellerebene, demnach vermutlich $\$ 2,4 \mathrm{Mrd}$. im Einzelhandel, und bei $50 \$$ pro Artikel und 310 Tagen pro Jahr setzt ein einzelner Laden weniger als 40 Artikel am Tag ab - eine leicht manuell erfaßbare Menge. Aus der täglichen Rückmeldung der Agenten lernt Benetton schon früh in der Saison, welche Farben gefragt sind. Der überwiegende Teil der Wollmode ist unifarben, wird grau gestrickt und dann während der Saison gefärbt. Durch dieses Vorgehen kann Benetton einen vom allgemeinen Modetrend relativ eigenwilligen (Farb-)Akzent setzen, der nah am Markt liegt und das Risiko von erheblichen Überbeständen begrenzt. Die Wettbewerbsposition von Benetton ist zweifellos stabil. Diese Stabilität gründet aber wohl nicht allein auf dem KIS. Benettons Einzigartigkeit mag durchaus auf die globale Kommunikationsfähigkeit und Anpassungmöglichkeiten zurückführbar sein, die bislang nicht imitiert wurden. Die Stabilität der Wettbewerbsposition gründet dabei aber auch auf völlig anderen Faktoren, etwa der Qualität, einschließlich der Kommunikation, und einer durch niedrige Integrationskosten effizienten Produktion. Benetton ist es zudem gelungen, die Stärken seines Grundgeschäfts (Farben, hohes Volumen, viele Läden, Globalität) mit einer geschickten kontraktuellen und technischen Geschäftsnetzwerkintegration zu kombinieren. Der Befund ähnelt dem der Geschäftsprozeßintegration und der internen Integration: Stabilität oder allgemeine Dauerhaftigkeit der Wettbewerbsposition ergibt sich aus einer Abstimmung des Grundgeschäfts mit der Integrationsstrategie. Gelingt es, nicht (leicht) imitierbare Elemente des Grundgeschäfts mit der Integrationsstrategie zu unterstützen, kann ein stabiler Wettbewerbsvorteil verstärkt werden, d.h. Stabilität für die focal Firm geschaffen werden durch elektronische vertikale Verknüpfungen $\mathrm{zu}$ einem vertikalen Netzwerk.

Die Stabilität würde direkt erhöht, wenn die Bildung eines vertikalen Netzwerks zu einem strategischen Commitment würde. Ein strategisches

berichten Johnston, H.R., Lawrence, P.R., Vertical Integration, a.a.O., S. 96. 
Commitment wurde definiert als das Eingehen einer Investition in starre Faktoren mit einer derart hohen Bindungswirkung, daß eine strukturelle Mobilitätsbarriere gegen Imitation geschaffen wird und der Versuch der Imitation ein glaubwürdiges Androhen von Vergeltungsmaßnahmen nach sich zöge, z.B. eine aggressive Preisstrategie. Beispielsweise ist Boeing als Hersteller des größten Jetflugzeugs ein Commitment von mehreren Milliarden US-Dollar eingegangen. Bei Ankündigung einer Imitation könnte Boeing glaubhaft androhen, die Preise bis zu den Grenzkosten zu senken. Dies würde einen Konkurrenten, der ein solches Commitment noch nicht eingegangen ist, und dessen Grenzkosten somit ungleich höher sind, vom Markt fernhalten. In keinem der untersuchten Beispiele konnte die Bildung eines vertikalen Netzwerks als strategisches Commitment qualifiziert werden. Der Versuch, mit vertikalen Netzwerken Mobilitätsbarrieren aufzubauen, erfolgt selten allein auf Basis hoher, versinkender Investitionen, sondern wesentlich direkter über das Anstreben von Zugangsvorteilen, wie z.B. bei Baxter oder den Flugreservierungssystemen.

Zwar kann die Einführung eines vertikalen Netzwerks die Stabilität der Position nicht erhöhen, zumindest aber auch nicht senken. Dies belegt der Fall von McKessons Economost. McKessons Kunden waren unabhängige Drogerien. Ohne einen so effizienten Großhändler wie McKesson hätten jene gegen die Drogeriemarktketten kaum bestehen können. ${ }^{1}$ Insofern sicherte McKesson durch ein erfolgreiches Bestellsystem auch die eigene Stabilität. Gleichwohl bestand in Bergen-Brunswick ein imitierender Konkurrent, der seinerseits ein Bestellsystem einführte. Beide Wettbewerber haben "neue Regeln" für ihre Branche eingeführt, aber keine überragenden Wettbewerbsvorteile gegeneinander erringen können - allerdings Wettbewerbsvorteile gegenüber kleineren Großhändlern, die den neuen Regeln nicht gefolgt sind. Dies kann auch an kooperativen Netzwerken aufgezeigt werden.

1 Clemons, E.K., Row, M., McKesson, a.a.O., S. 45; Johnston, H.R., Lawrence, P.R., Vertical Integration, a.a.O., S. 94 ff. 
An vertikalen Netzwerken aus der Reifenindustrie, der Lebensmittelbranche oder dem Bürobedarf kann gezeigt werden, daß die Bildung vertikaler Netzwerke häufig mehr aus Notwendigkeit denn zum Erreichen eines Vorteils gebildet werden. ${ }^{1}$ Die Notwendigkeit kann sich dabei auch aus dem vertikalen Geschäftssystem selbst ergeben, d.h. ein Lieferant/Abnehmer setzt die Einrichtung der elektronischen Verknüpfung durch. ${ }^{2}$

Selbst ein hoher Marktanteil muß nicht notwendigerweise Stabilität begründen, wenn er mit elektronischen vertikalen Verknüpfungen unterlegt wird. Prinzipiell bildet ein hoher Marktanteil, wie die Fallbeispiele von Frito Lay bis Citibank verdeutlichen, eine tragfähige Basis zum Ausbau der Wettbewerbsposition durch den Einsatz von KIS, insbesondere wenn damit Zugangsbeschränkungen zum Markt aufgebaut werden. Die Vorteile können jedoch durch eine Defensivstrategie der Wettbewerber, nämlich die Einführung kooperativer Systeme, neutralisiert werden. ${ }^{3}$ Dies geschah bei den beiden in den USA bedeutendsten Flugreservierungssystemen, United Airlines Apollo und American Airlines SABRE. Beide hatten eine ähnliche Plazierungsdichte in Reisebüros erreicht, United jedoch den höheren Marktanteil und damit das breitere Angebot. Daraufhin entschloß sich American Airlines im Jahr 1978, fünf weiteren Fluggesellschaften eine Buchung über SABRE anzubieten. Damit sollte die Breite des Sortiments erweitert und die Attraktivität von SABRE gegenüber den Reisebüros erhöht werden. United entschloß sich umgehend, seinerseits weitere Gesellschaften auf Apollo Zugang zu gestatten. Insgesamt war allerdings der Vorsprung von United aus einem breiteren Leistungsprogramm neutralisiert. 4

Kooperative Systeme werden aber nicht nur durch Wettbewerb erzwungen. So haben die europäischen Fluggesellschaften kooperative Reservierungssysteme von vornherein gemeinsam entwickelt und eingeführt, 5 ebenso

1 Vgl. Benjamin, R.I., DeLong, D.W., Scott Morton, M.S., Electronic Data Interchange, a.a.O., S. $10 \mathrm{ff}$.

2 Z.B. Reynolds Tobacco Corp. oder die Zulieferer von McDonnell Douglas, vgl. Kolodziej, S., Success, a.a.O., S. 37.

3 Vgl. die Reaktion auf die Einführung von ATMs durch Citibank..

4 Vgl. Copeland, C.G., McKenney, J.L. Airline Reservation, a.a.O., S. 361.

5 Dies sind die Systeme Amadeus, basierend auf der Software von United Airlines, und Galileo, basierend auf den System One von Texas Air. 
die finnische Papier- und Pappindustrie ein gemeinsames, weltumspannendes Bestellsystem ${ }^{1}$ und eine Gruppe amerikanischer Sachversicherer ein System, das elektronische vertikale Verknüpfungen zu den unabhängigen Agenturen erlaubt. ${ }^{2}$ Diese haben freilich aus Sicht der einzelnen Unternehmung den Nachteil, daß weder eine spezifische Abstimmung mit dem Grundgeschäft noch eine Differenzierung durch das System möglich sind. Beispielsweise partizipierten zahlreiche amerikanische Sachversicherer nicht am System IVANS, weil sie sich nicht mehr durch eigene Systeme, u.a. Expertensysteme, gegenüber den Agenturen differenzieren, sondern nur noch über den Preis konkurrieren könnten. ${ }^{3}$

\subsubsection{Dauerhaftigkeit in vertikalen Netzwerken: Zusammenfassung}

Vertikale Netzwerke zwischen Unternehmungen ermöglichen in vielen Beispielen beträchtliche Positionsvorteile, direkt durch Verkürzung von Abwicklungszeiten, indirekt durch Verbesserung der Kosten- und Qualitätsposition. Dabei kann die Stabilität der Position für die "focal Firm" nicht allein aufgrund der Integrationsstrategie, sondern nur durch eine Kombination der Integrationsstrategie mit dem Grundgeschäft aufgebaut werden.

Auch die Bildung elektronischer vertikaler Netzwerke kann als Gefangenendilemma angesehen werden, d.h. als notwendiges Commitment, das einzugehen ist, auch wenn die daraus entstehenden Vorteile nicht stabilisierbar sind. Sie sind nicht stabilisierbar, weil die Potentiale einer Integrationsstrategie sowohl imitierbar als auch endlich sind, d.h. der Grenznutzen der Integration nimmt rasch ab. Beispielsweise zog im Fallbeispiel Baxter die Modifikationen von ASAP 4 bis ASAP 8 kaum nennenswerte Positionsvorteile nach sich. Trotz mangelnder Stabilisierbarkeit sind Commitments in die elektronischen vertikalen Netzwerke notwendig. Es besteht angesichts hoher kompetitiver Interdependenz nicht die Option, ein mögliches vertikales Netzwerk nicht einzurichten.

1 Vgl. Reponen, T., Copeland, D. McFarlan, F.W., Finnpap/Finnboard (A), Boston, Massachusetts, Harvard Business School Case No. 9-186-130, 1985; Mills, R.G., Reponen, T., McFarlan, F.W., Finnpap/Finnboard (B) Fin-Project, Boston, Massachusetts, Harvard Business School Case No.9-188-103, Revised July 1988.

2 Vgl. Warbelow, A., Konsynksi, B., IVANS, Boston, Massachusetts, Harvard Business School Case No. 9-187-188, Revised July 1987.

3 Vgl. Venkatraman, N., Zaheer, A., Electronic Integration, a.a.O., S. 4 f. 
Vertikale Netzwerke verbinden die Vorteile vertikaler technischer und organisationaler Integration ohne die Nachteile vertikaler kontraktueller Integration. Auf Geschäftssystemebene sind vertikale Netzwerke den vertikal integrierten Unternehmungen so stark überlegen, daß letztere kaum erfolgreich gegen vertikale Netzwerke bestehen können. Die schwache Profitabilität von Baxter im Vergleich zu Johnson \& Johnson und Abbott mag ein Beleg für diese These sein, die Rentabilitätsprobleme der kontraktuell vertikal tief integrierten europäischen und amerikanischen Automobilhersteller im Vergleich zu den japanischen oder die unangegriffene Position des Netzwerks von Benetton zusätzliche Belege. Ein einfacher, aber wichtiger Vorteil der vertikalen Netzwerke sind die wesentlich geringeren Integrationskosten, insbesondere Agencykosten, durch dezentral distribuierte Eigentums- und Kontrollrechte.

Dieser Befund entspricht den Erkenntnissen von Venkatraman und Short, die einen evolutorischen Pfad der elektronischen Integration beschreiben (vgl. Abb. C.2.13).

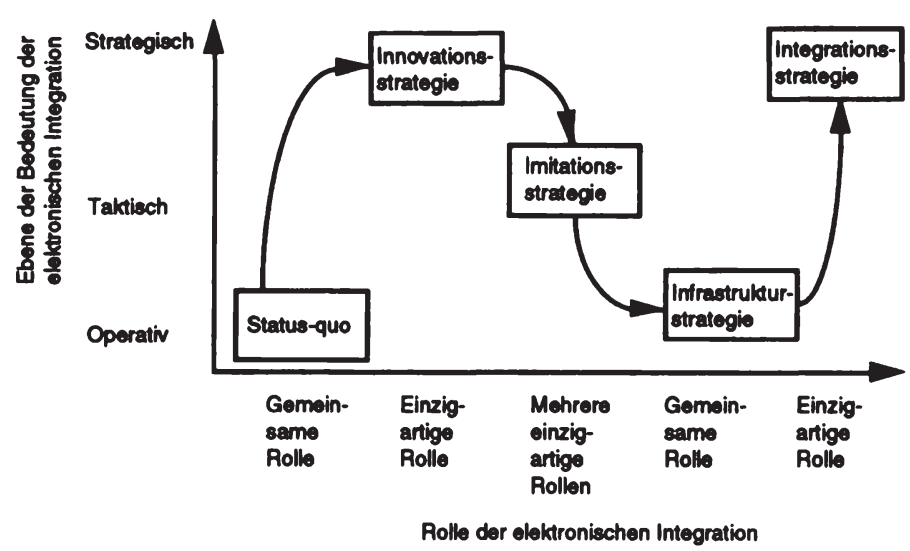

Abb. C.2.13: Evolutionsmuster von Strategien der eloktronischen Integration

Quelle: Venkatraman, N. Short, J.E., Strategies for Electronic Integration, Author's Draft, Massechusetts Institute of Technology, 1980, S. 27

Dabei stellen sie die Bedeutung von Maßnahmen zur elektronischen Integration (qualifiziert als operativ, taktisch und strategisch) und ihre Rolle innerhalb einer Strategie (einzigartig oder nicht) im Zeitverlauf dar. In die- 
sen evolutorischen Pfad sind auch Konkurrenzsituationen einbezogen. Wenngleich die Dimensionen des in dieser Arbeit vorgelegten Bezugsrahmens bei Venkatraman und Short vermengt werden, (Wettbewerbsposition und Dauerhaftigkeit sind verschiedene, sogar konfliktäre, Teildimensionen der strategischen Bedeutung) bildet der Pfad das Gefangenendilemma aus dem Eingehen von Commitments ab: Ausgehend von einer Situation, in der keine elektronische Integration vorliegt, hat die sogenannte Innovationsstrategie eines First Mover strategische Bedeutung (= Positionsvorteile), die dann durch die Imitation abschmilzt, (= Stabilitätsprobleme) und schließlich zur "allgemein verfügbaren" Infrastruktur wird (keine Positionsvorteile mehr). Eine hohe Bedeutung (= Positionsvorteile) kann dann nur noch durch die sogenannte "Integrationsstrategie" gewonnen werden. Als Integrationsstrategie definieren Venkatraman und Short die im folgenden als Information Partnerships charakterisierte Nutzung von Informationen. ${ }^{1}$ Beispielsweise verfügen die Betreiber der Flugreservierungssysteme über eine erhebliche Datenbasis, mit der wichtige Erkenntnisse über den Markt und die Wettbewerber gewonnen werden können. McKesson verfügt über sehr genaue Abverkaufsdaten für Drogerieartikel, desgleichen Baxter über Krankenhausbedarf. Selbst können die genannten Unternehmen diese Daten nur zum Teil nutzen, z.B. für ihre eigene Planung, wie etwa Frito Lay eine "Echtzeit-Marktforschung" betreiben kann.

Detaillierte Informationen über Märkte entstehen als "Kuppelprodukt" von vertikalen Netzen. Beispielsweise kennt ein Träger eines vertikalen elektronischen Retailing-Netzes die genauen Abverkaufszahlen verschiedener Marken in verschiedenen Regionen, bei der Nutzung von Scanner-Kassen sogar in Echtzeit. Derartige Informationen sind für die Konsumgüterhersteller von hohem Wert, wie die hohen Budgets für (KontrollMarktforschung zeigen, die im Vergleich wesentlich schlechtere Daten liefert, und zwar im Hinblick auf Aggregation, Genauigkeit und Aktualität. Allerdings kann zur "Echtzeit-Realdaten-Marktforschung" mit vertikalen Netzwerken lediglich die Beobachtung, nicht die Befragung eingesetzt werden. Ein anderes Beispiel für Informationen hohen Werts ist die in den USA sich abzeichnende elektronische Einkommensteuererklärung. Im vertikalen Netzwerk zwischen Steuerberatungsgesellschaften, Steuerzahlern und Internal Revenue Service (IRS) entstehen Informationen, die ein "Segement of One"-Marketing für Finanzdienstleistungen erlauben wür-

1 Vgl. Venkatraman, N., Short, J.E., Electronic Integration, a.a.O., S. 29. 
den, also nach Einkommens- und Vermögenssituation maßgeschneiderte Anlage- und Kreditprodukte. ${ }^{1}$ Der tatsächliche Wert der aus vertikalen Netzwerken gewonnenen Information entsteht somit erst aus der Bildung (immaterieller) horizontaler Verflechtungen. Diese als Information Partnerships bezeichnete Integrationsstrategie ist Gegenstand des folgenden Abschnitts.

\subsubsection{Information Partnerships}

Information Partnerships ${ }^{2}$ sind kontraktuell nicht integrierte Unternehmungen, die materielle und immaterielle elektronische horizontale Verflechtungen zueinander unterhalten. Träger einer Information Partnership ist dabei nicht eine focal Firm wie im vertikalen Netzwerk, sondern eine Gruppe von Unternehmen. Die Information Partnerships richten sich nicht in erster Linie auf die Abwicklung des Geschäftsprozesses der beteiligten Unternehmen, sondern vielmehr auf den Informationstausch, d.h. die Bildung immaterieller horizontaler Verflechtungen zwischen gleichartigen Geschäftsaktivitäten, z.B. die gemeinsame Nutzung oder gar Schaffung von Datenbanken. ${ }^{3}$ Dies entspricht der im Rahmen der vertikalen Netzwerke diskutierten "Zweitverwertung" von Informationen durch den Träger eines vertikalen Netzwerks, indem jene Informationen mit Unternehmungen außerhalb des vertikalen Netzwerks ausgetauscht werden. Mate-

1 Dabei ist fraglich, ob die Nutzung dieser Informationen tatsächlich wünschenswert ist. Aus Kundenperspektive mag es durchaus interessant sein, für die eigene Steuer- und Vermögenssituation maßgeschneiderte Finanzdienstleistungen angeboten zu bekommen. Fraglich erscheint jedoch, inwieweit der Kunde dem Träger der elektronischen Steuererklärung das Recht einräumen wird, die Informationen an Finanzdienstleister weiterzugeben. Auch die Lösung daB Finanzdienstleister Träger der elektronischen Steuererklärung würden, erscheint unvollkommen, da aus Kundenperspektive hier häufig ein Vertrauensverhältnis zu einem unabhängigen Berater präferiert wird. Möglicherweise ist das Thema der Datenhingabe aus deutscher Perspektive ein sensibleres als aus amerikanischer. Zur elektronischen Einkommensteuererklärung vgl. Venkatraman, N., Kambil, A., Electronic Integration, a.a.O., bes. S. 37 f.

2 Vgl. Konsynski, B.R., McFarlan, F.W., Information Partnerships, a.a.O., S. 114. Der Begriff Partnership ist schwer mit einem Wort übersetzbarer; er kann als Partner- und Teilhaberschaft, Beteiligung, und Handelsgesellschaft verstanden werden, insofern enthält der Begriff eine kooperative und eine formale Dimension. Insbesondere löst der deutsche Begriff "Partnerschaft" unzutreffende Konnotationen aus. Partnership bleibt daher unübersetzt. Konsynski und McFarlan verstehen Information Partnerships synonym mit der Geschäftsnetzwerkintegration. Demgegenüber werden hier als Information Partnerships nur im wesentlich auf den Informationsaustausch abstellende Integrationsstrategien, nicht jedoch auf die tatsächliche Abwicklung bezogene Integrationsstrategien angesehen.

3 Vgl. Malone, T., Yates, J., Benjamin, R. I., Electronic Markets, a.a.O., S. 495 ff. 
rielle elektronische horizontale Verflechtungen werden durch die gemeinsame Nutzung gleichartiger Faktoren gebildet.

\subsubsection{Ansätze und Wirkungsweisen}

Ein Beispiel für Information Partnerships im Reisemarkt ist in Abb. C.2.14 grob skizziert. United Airlines, Hertz Car Rental und Westin Hotels, kontraktuell nicht integrierte Unternehmen, nutzen gemeinsam das Reservierungssystem von United - eine materielle horizontale Verflechtung.

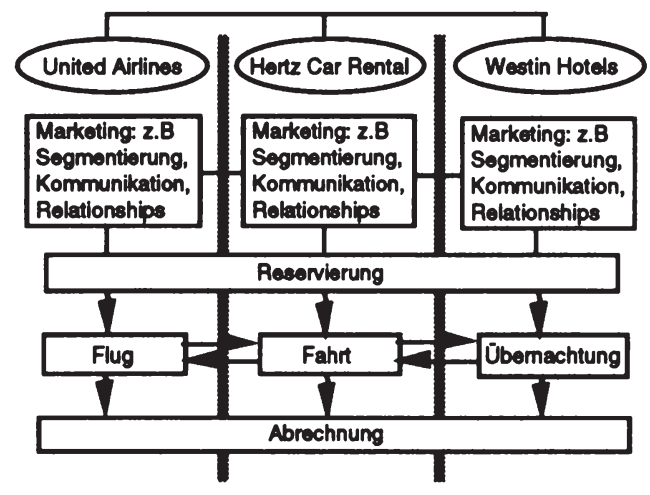

Abb. C.2.14: Horizontale Verflechtungen im Reisemarkt

Die Bildung mehrerer immaterieller Verflechtungen ergibt sich aus dem Austausch von Kundendaten in der Segmentierung, einer abgestimmten Kommunikationspolitik in den Kundensegmenten sowie der Nutzung jeweiliger Kontakte zu Großkunden. Im eigentlichen Geschäftsprozeß selbst bestehen nur schwache Potentiale zur vertikalen Verknüpfung: So können einem Kunden, der einen United-Flug bucht, ein Hertz-Mietwagen und ein Westin-Hotelzimmer angeboten werden. Durch ein gemeinsames KIS ist es aber z.B. möglich, den tatsächlichen Reiseantritt des Kunden festzustellen und dementsprechend den Mietwagen bereitzustellen und das Einchecken im Hotel vorzubereiten.

Der Zusammenarbeit von United, Hertz und Westin ging der Versuch voraus, alle drei in einer Firma (Allegis) kontraktuell zu integrieren. Dieser Versuch ist gescheitert. Die punktuelle Zusammenarbeit durch materielle 
und immaterielle elektronische Verflechtungen muß keineswegs durch die kontraktuelle Integration abgesichert werden, vielmehr erscheinen relationale Verträge hinreichend. Dies kann darauf zurückgeführt werden, daß aufgrund der Kooperation der drei Beteiligten im wesentlichen keine Commitments in fixe Faktoren zu tätigen waren. KIS waren zur Abwicklung des jeweils eigenen Geschäfts ohnehin eingerichtet, ihre technische Integration war (ökonomisch) unproblematisch, d.h. sie zog keine spezifischen Investitionen in starre Faktoren nach sich. Die kontraktuelle Integration der drei Einheiten zu Allegis führte zu einem (kontraktuell) zu hohen Integrationsgrad. Hohe Integrationskosten (Nachlässigkeitskosten), sichtbar im unbefriedigenden Ergebnis des operativen Geschäfts, hohe Administrationskosten durch höheren Aufwand für Overhead und negativer Integrationsnutzen wegen Positionierungsschwierigkeiten (die Marke Allegis konnte nicht etabliert werden) führten dann auch zum Abbruch der kontraktuellen Integration bei Ausbau der punktuellen, technischen Integration. ${ }^{1}$

\subsubsection{Wettbewerbsposition und Dauerhaftigkeit}

Es ist fraglich, welchen Beitrag die elektronische horizontale Verflechtung zur Erlangung und Sicherung eines Wettbewerbsvorteils für die Beteiligten leistet. Information Partnerships erscheinen weit weniger dramatische Positionsgewinne nach sich zu ziehen, als dies in den Beispielen vertikaler Netzwerke der Fall war. So bieten inzwischen nahezu alle Träger von Flugreservierungssystemen umfangreiche Hotel- und Mietwagenprogramme an, einschließlich umfangreicher Textinformation und farbiger Bildpräsentation über VHS-Videosysteme. ${ }^{2}$

Demgegenüber scheint ein jüngerer Fall einer Information Partnership größere Positionsgewinne zu ermöglichen. Dies war die Entscheidung von

1 Konsynski und McFarlan werfen analog die Frage der Sinnhaftigkeit von "Allfinanz"Konzernen aus Banken und Versicherungen auf. Vgl. Konsynski, B.R., McFarlan, F.W., Information Partnerships, a.a.O., S. 114. Am 25. Mai 1991 gab die Dresdner Bank AG bekannt, daB sie $10 \%$ von der Allianz Versicherung erworben habe und eine umgekehrte Beteiligung ebenfalls vorliege. Vgl. Fisher, A., Dresdner Confirms Allianz Holding, in: Financial Times, 25. Mai 1991, S. 10.

2 Vgl. Henderson, B., The Experience Curve Reviewed, IV. The Growth Share Matrix of the Product Portfolio, Perspectives, No. 135, Boston, MA: The Boston Consulting Group, 1989, S. 54 ff. 
American Telephone \& Telegraph, die Basis von 60 Mio. Telefonkarten simultan als "Universal Card", d.h. als kombinierte Kredit- und Telefonkarte zu nutzen. Ein entscheidender Positions- und Stabilitätsvorteil im Markt für Kreditkarten ist der Marktanteil. Dieser ist neben der Gebühr der Kartenorganisation entscheidender Einflußfaktor für die Akzeptanz der Karte bei den Zahlungsempfängern. Umgekehrt ist der Kartenkunde zur besseren Handhabbarkeit an einer möglichst überschaubaren Anzahl an Kredit- und Telefonkarten interessiert und wird versuchen, mit seinen Karten möglichst alle potentiellen Nutzungsorte abzudecken. ${ }^{1}$ Insgesamt sind Skaleneffekte damit eine entscheidende Determinante der Qualitätsposition, gleichzeitig auch der Kostenposition. Die Universal Card ist eine materielle horizontale Verflechtung der Kundenbasis von AT\&T und MasterCard. Beide gewinnen Positionsvorteile und, zumal bei dem allein von AT\&T eingebrachten Volumen, Stabilitätsvorteile. Die Einführung blieb allerdings nicht unbeantwortet. In einer Information Partnership von Visa, Citibank und der Telefongesellschaft MCI wurden aus 14,6 Millionen VisaKarten nun simultan Bank- und MCI Karten. ${ }^{2}$ Der Marktanteil von AT\&T/Mastercard ist wohl kaum kopierbar. Diese Beispiele haben viele andere Information Partnerships angeregt, unter anderem zwischen VISA, der Berliner Bank und der Umweltkarte der Berliner Verkehrsbetriebe.

Zusammenfassend kann erneut der Schluß gezogen werden, daß durch strategische Integration mit Informationstechnologie Positionsvorteile aufgebaut werden können, deren Dauerhaftigkeit jedoch durch inhärente Stabilitäts- und Verteilungsprobleme gefährdet ist. Auf Geschäftssystemebene können erhebliche Verbesserungen erzielt werden, die jedoch der Imitation durch die Wettbewerber und der Abschöpfung durch den Kunden unterliegen. Dauerhafte Wettbewerbsvorteile sind auch im Falle von Information Partnerships nur möglich, wenn die Logik des Grundgeschäfts mit derjenigen der Integrationsstrategie gut übereinstimmt, wie etwa im Fall von der Universal Card von AT\&T.

1 Die Zahlung von Kartenkonten in den USA erfolgt per Scheck, d.h. der Kunde muß für jede einzelne Telefon- und Kreditkarte monatlich einen eigenen Scheck ausschreiben und versenden.

2 Konsynski, B.R., McFarlan, F.W., Information Partnerships, a.a.O., S. 116. 


\subsubsection{Elektronische Märkte}

Elektronische Märkte können als Netzwerke angesehen werden, die von einem Marktträger (Market Maker) getragen werden, der selbst nicht Teil des eigentlichen Geschäftsprozesses ist. Die Charakterisierung eines Netzwerks als Markt weist bereits darauf hin, daß die Beziehungen der Marktteilnehmer nicht durch kontraktuelle Integration bzw. firmenkonstituierende Kontrakte geknüpft sind, sondern durch Marktkontrakte und marktorientierte relationale Kontrakte. Die Beziehungen der Marktteilnehmer zum Marktträger werden dabei oft durch langfristigere relationale Kontrakte geregelt. Beispiele für elektronische Märkte sind Märkte für Automobilzubehör ${ }^{1}$, gebrauchte Lehrbücher ${ }^{2}$, gebrauchte Automobile ${ }^{3}$, Diamanten $^{4}$ und Versicherungen 5 .

\subsubsection{Integrationsbereich elektronischer Märkte}

Transnet ist ein elektronischer Markt für Automobilzubehör in den USA. Der Markt für Automobilzubehör ("Aftermarket") hat, je nach Definition ein Volumen von $\$ 65$ - 90 Mrd. Marktträger von Transnet war ein Verband der Hersteller. Durch Transnet waren 100 Hersteller mit 4500 Abnehmern, Händlern, Tankstellen usw. verbunden. 1987 wurden 70 Mio. Teilepositionen über Transnet gehandelt. Dabei hatte der Käufer den jeweiligen Hersteller zu spezifizieren, die Teile-Nummer sowie einen Preiscode anzugeben. Dieser war mit dem Hersteller auszuhandeln. ${ }^{6}$ Transnet ist zwar prinzipiell als elektronischer Markt anzusehen, doch wesentliche Marktfunktionen, insbesondere die Bestimmung des Verkäufers, Preisvergleiche und Preisverhandlungen, wurden außerhalb von Transnet ausgeführt.

1 Vgl. McGee, J.V., Konsynski, B., Transnet, Boston, Massachusetts, Harvard Business School Case No. 9-188-098, 1988.

2 Vgl. Vitale, M.R., Ives, B., MBS Textbook Exchange, Boston, Massachusetts, Harvard Business School Case No. 9-188-028, 1988.

3 Vgl. Warbelow, A., Kokuryo, J., Konsynski, B., Aucnet TV Auction Network System, Boston, Massachusetts, Harvard Business School Case No. 9-190-001, Rev. 19.7.89, 1989.

4 Vgl. Warbelow, A., Konsynksi, B., American Gem Market System, Boston, Massachusetts, Harvard Business School Case No. 9-189-088, 1988.

5 Vgl. Venkatraman, N., Zaheer, A., Electronic Integration, a.a.O., S. 4; Warbelow, A., Konsynksi, B., IVANS, a.a.O.

6 Vgl. McGee, J.V., Konsynski, B., Transnet, a.a.O., S. 1 - 7. 
Von 4,4 Mio. Gebrauchtwagentransfers in Japan im Jahr 1987 wurden 0,7 Mio. über Auktionen verkauft, 25.455 davon über einen 1985 gegründeten elektronischen Markt, Aucnet. In wöchentlichen Auktionen wurden zuvor inspizierte Gebrauchtwagen zum Kauf angeboten. Marktteilnehmer, insbesondere auf Seite der Käufer und Warenhändler. Gebote wurden in Inkrementen von $¥ 3.000$ abgegeben, das Höchstgebot erhielt den Zuschlag, wenn es ein vom Käufer gesetztes Limit überstieg, welches jener auch während der Auktion noch ändern konnte (vgl. Abb. C.2.15).

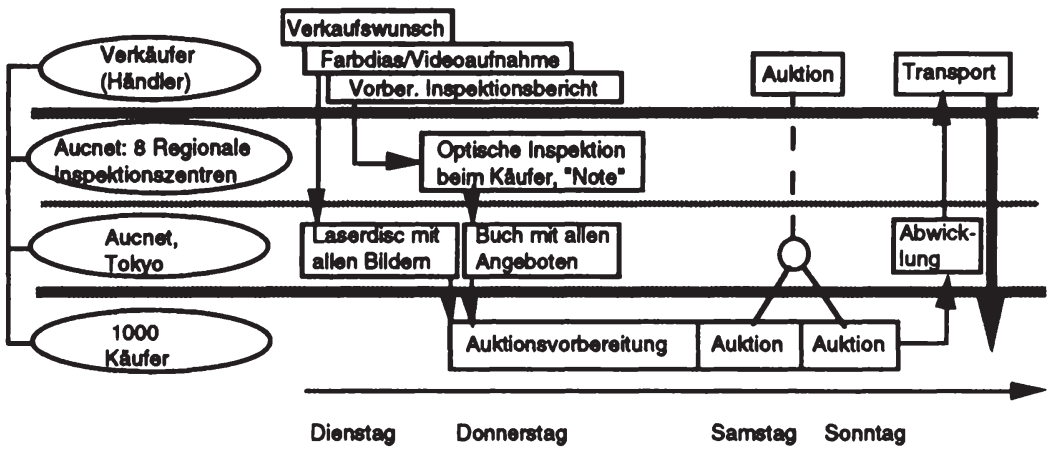

Abb. C.2.15: Aucnet elektronischer Gebrauchtwagenmarkt

Über Aucnet wurden überwiegend hochpreisige Gebrauchtautomobile gehandelt. Diese wiesen eine gegenüber älteren Automobilen geringere asymmetrische Informationsverteilung ${ }^{1}$ zwischen Verkäufer und Käufer auf und konnten wie Neuwagen eher "unbesehen" erworben werden. Der Vorteil elektronischer Märkte, Transaktionen zwischen räumlich entfernten Marktteilnehmern, begründet den Nachteil, den Tauschgegenstand nicht unmittelbar prüfen zu können. Diesem Problem trat der Marktträger Aucnet teilweise durch Inspektionen entgegen, die zusammen mit den hohen japanischen Sicherheitsvorschriften und dem Marktsegment fast neuer Wagen zum Erfolg Aucnets beitrugen. Aucnet verzeichnete bereits 3 Jahre nach Gründung einen Provisionsumsatz von $¥ 1900 \mathrm{Mio}$. und einen Gewinn von $¥ 300$ Mio. Mehrere Versuche, Aucnet $z$ imitieren, darunter ein kooperativer Ansatz des Verbandes der Automobilauktionatoren und

1 Vgl. Akerlofs klassischen Beitrag zum Problem der "Lemons": Akerlof, G.A., The Market for Lemons: Quality Uncertainty and the Market Mechanism, in: Quarterly Journal of Economics, Vol. 84, 1970, S. 488; ein deutscher Überblick ist Spremann, K., Zur, E., (Hrsg.), Informationstechnologie, a.a.O., hier besonders S. 564 ff. 
ein lokaler geschlossener Auktionsmarkt, scheiterten, weil sie nicht das notwendige Volumen erlangen konnten. Der Anzahl elektronischer Märkte sind Grenzen gesetzt, da die bei den Händlern jeweils erforderliche Ausrüstung (Personal Computer, Farb-TV, Satellitenempfang oder Videodisk) teilweise systemspezifisch ist. ${ }^{1}$

Auch in anderen elektronischen Märkten löst der Marktträger das Problem der asymmetrischen Informationsverteilung zwischen Verkäufer und Käufer. In einem elektronischen Markt für Diamanten hatte der Marktträger American Gem Market System ein Rating-System für Diamanten entwikkelt, das umfassender war als das herkömmliche. Zudem wurde angeboten, eingesandte Steine unabhängig zu klassifizieren und die Beschreibung des Steins dauerhaft zu speichern. Der Marktträger bot viele weitere Dienstleistungen an, insbesondere Datenbankanalysen vergangener Marktbewegungen vergleichbarer Steine. Die Marktteilnehmer konnten wahlweise öffentliche und geschlossene Märkte einrichten, wobei geschlossene Märkte von Anbietern bevorzugt wurden, die ihre Preisgestaltung anderen Anbietern nicht offenbaren wollten. Über das System wurden vor allem kleinere Steine gehandelt, für die die Transaktionskosten gegenüber dem sonst üblichen Versand zur Ansicht bzw. der Reise des Einkäufers gesenkt werden konnten. ${ }^{2}$

MBS Textbook Exchange war ein elektronischer Markt für gebrauchte Lehrbücher. Der Markt für Lehrbücher in den USA hatte 1988 ein Volumen von $\$ 2$ Mrd. (Einzelhandelspreise), derjenige für gebrauchte Lehrbücher ein Volumen von $\$ 200$ Mio. Die Universitätsbuchhandlungen in den USA boten den Studenten zu Semesterbeginn Lehrbücher neu und gebraucht nach Kursen geordnet an. Am Semesterende kauften die Buchhandlungen gebrauchte Lehrbücher zurück, die zu $50 \%$ des Einzelhandelspreises gehandelt wurden, wobei das Angebot gefragter Lehrbücher chronisch knapp war. MBS hatte einen elektronischen Markt für ca. 35.000 der 64.000 Lehrbuchtitel eingerichtet. Universitätsbuchhandlungen konnten ihr Kaufinteresse an gebrauchten Lehrbüchern eingeben. Damit konnten die Marktpreise der Bücher nach Angebots- und Nachfragesituation ermittelt werden. Dennoch war der Markt chronisch "illiquide", d.h. die Nachfrage

1 Vgl. Warbelow, A., Kokuryo, J., Konsynski, B., Aucnet TV Auction Network System, a.a.O., S. 6 ff.

2 Vgl. Warbelow, A., Konsynksi, B., American Gem Market System, a.a.O., S. 5 ff. 
übertraf das Angebot bei weitem. MBS bot den Buchhandlungen TEXT-AID an, ein Abwicklungssystem, das die Bestellung von Lehrbüchern, die Organisation der Lehrbuchabteilung und den Rückkauf von Lehrbüchern unterstützt. Fast jede Buchhandlung war mit der rechtzeitigen Buchbestellung und Organisation der Lehrbuchabteilung überfordert. ${ }^{1}$ Als Kompensation für die Überlassung der TEXT-AID-Software verlangte MBS jährlich von jeder teilnehmenden Buchhandlung gebrauchte Lehrbücher mit einem Volumen von $\$ 25.000$ in das System zu geben. Die Vereinbarung wurde vielseits rasch angenommen. Damit war MBS in der Lage, den Markt "liquide" zu halten. MBS erzielte 1987 auf einen Umsatz von \$54 Mio. einen Gewinn von $\$ 8,7$ Mio. ${ }^{2}$

Instinet war ein interaktives Aktieninformations- und Handelssystem, das als Marktteilnehmer institutionelle Anleger, Broker und Händler ("Specialists") verbindet. ${ }^{3}$ Gegenstand von Instinet waren 8000 Werte, die ansonsten im Telefonhandel unter Brokern gehandelt wurden. Die Transaktionskosten über Instinet beliefen sich auf $3 €$ pro Aktie, während sonst 8 § üblich waren. 1985 hatte Instinet 10.900 Marktteilnehmer, mit denen es beträchtliche Umsätze in einigen wichtigen Nebenwerten erzielte. Entscheidende Hürde auch für Instinet war die Marktliquidität, denn Instinet war für die Marktteilnehmer erst dann von Bedeutung, wenn $20-25 \%$ des Handels mit einem Wert auf dem Netz erreicht wurden. Der Marktträger, eine For-Profit-Aktiengesellschaft, arbeitete bis 1986 gerade kostendeckend. Größere Minderheitsbeteiligungen hielten Reuters und einige Brokerfirmen. 4

Der Träger eines elektronischen Marktes für Sach- und Haftpflichtversicherungen IVANS (Insurance Value Added Network Services) ist eine Non-Profit-Unternehmung, die durch einen Branchenverband gegründet wurde, um zwischen ihnen und unabhängigen Agenturen als Marktteil-

1 Die Universitätsbuchhandlungen in den USA haben Lehrbuchabteilungen, die nach Kursen sortiert Lehrbücher anbieten. Die Bestellung der Lehrbücher ist dabei sehr kompliziert. Das hat vielfältige Gründe: Die Bestellfristen betragen mangels eines Grossistensystems 4-6 Wochen. Professoren geben eine Liste mit ca. 1 - 20 Lehrbüchern für einen Kurs an. Dabei werden gleiche Lehrbücher für verschiedene Kurse benutzt und verschiedene Professoren benutzen verschiedene Lehrbücher für den gleichen Kurs etc.

2 Vgl. Vitale, M.R., Ives, B., MBS Textbook Exchange, Harvard Business School Case No. 9-188-028, Boston, 1988.

3 Vgl. Bodurtha, St., G., Perold, A.F., Instinet Corporation (A), Boston, Massachusetts, Harvard Business School Case No. 1-286-116, Revised Version, Boston 1988, S. 1 und $12 \mathrm{ff}$.

4 Vgl. Bodurtha, St., G., Perold, A.F., Instinet Corporation, a.a.O. S. 13 ff. 
nehmer einen elektronischen Markt für Sach- und Haftpflichtversicherungen zu bilden. Die Hälfte des Volumens der Branche von $\$ 100 \mathrm{Mrd}$. wurde durch 50000 kontraktuell nicht integrierte Agenturen abgewickelt, die andere Hälfte durch den Außendienst der Versicherungen selbst. IVANS bildete dabei eine Alternative zu den vertikalen Netzwerken einzelner Gesellschaften. Ein unabhängiger Agent konnte über IVANS die Art des $\mathrm{zu}$ versichernden Risikos beschreiben und Prämieninformationen prinzipiell von allen Gesellschaften seiner Wahl einholen. Außerdem war über IVANS die Schadensbearbeitung laufender Versicherungen möglich. 1987 wickelten ca. 3500 Agenturen und ca. 30 Versicherungsgesellschaften ein Volumen von etwa $\$ 6$ Mrd. über IVANS ab. ${ }^{1}$

\subsubsection{Wettbewerbsposition in elektronischen Märkten}

Im Gegensatz zu den anderen Formen der Geschäftsnetzwerkintegration sind die Marktträger der elektronischen Märkte nicht selbst Teil des Geschäftsnetzwerks. Die Wettbewerbsposition eines Marktträgers ist daher nicht auf den Vergleich mit den Marktteilnehmern zu beziehen, sondern auf den Vergleich mit der Alternative zum elektronischen Markt. Als Ursprung der Entwicklung eines elektronischen Marktes kommen prinzipiell zwei Quellen in Frage: ein herkömmlicher Markt, dessen Effizienz durch ein KIS erhöht werden kann, oder ein zuvor kontraktuell eng integriertes Netzwerk, das durch verstärkten KIS-Einsatz kontraktuell de-integriert werden kann zu marktorientierten relationalen Kontrakten. ${ }^{2}$ Dabei liegen die kooperativen vertikalen Netzwerke in einem Grenzbereich zwischen elektronischen Märkten und vertikalen Netzwerken. Die Strategie der Kooperation wird durch Hereinnahme verschiedener Anbieter in das Netzwerk verfolgt, die gemeinsam die Marktträgerschaft übernehmen.

Ein elektronischer Markt hat Positionsvorteile gegenüber traditionellen Märkten durch niedrigere Transaktionskosten, weil das KIS ein Geflecht aus Brokern und Zwischenhändlern substituiert. Verbessert wird die Position durch Zeitvorteile im Handel und (Transaktions-)Kostenvorteile. Diesen Positionsvorteilen steht ein Positionsnachteil gegenüber, wenn eine physische Begutachtung des Tauschgegenstands erforderlich ist. Teilweise

1 Vgl. Warbelow, A., Konsynksi, B., IVANS, a.a.O., S. 1 ff.

2 Vgl. Malone, T., Yates, J., Benjamin, R.I. Electronic Markets, a.a.O., S. 490. 
können Marktträger diesen Nachteil durch eine neutrale Begutachtung des Tauschgegenstands begrenzen. Dennoch beschränkt dieses Problem etwa den elektronischen Handel von Gebrauchtwagen auf fast neue Wagen, denjenigen von Diamanten auf kleinere Steine.

Der zentrale Positionsvorteil elektronischer Märkte gegenüber vertikalen Netzen liegt in der Qualitätsposition. Mit einem elektronischen Markt sind die Marktteilnehmer nicht auf das Angebot einer focal Firm angewiesen, sondern können auf eine Fülle von konkurrierenden Anbietern zugreifen. Dies wird zu erheblicher wettbewerblicher Interdependenz in den Märkten führen und den Preis zu einem wichtigen Parameter machen. Daher werden auf Differenzierung gerichtete Anbieter, etwa im Versicherungsmarkt, dem elektronischem Markt fernbleiben und eigene vertikale Netzwerke anbieten. Dies ist jedoch nicht immer möglich, weil auch die vertikalen Netzwerke untereinander im Wettbewerb stehen und einen erheblichen Druck zur Degeneration zu elektronischen Märkten aufweisen.

\subsubsection{Degeneration vertikaler Netzwerke zu elektronischen Märkten}

Am Beispiel der Flugreservierungssysteme wurde bereits gezeigt, daß kooperative Netzwerke, quasi elektronische Märkte, oft als Defensiostrategie eingesetzt werden, um gegen geschlossene vertikale Netzwerke des Marktführers zu bestehen, die angesichts der Positions- und Dauerhaftigkeitsvorteile aus dem Grundgeschäft strukturelle Mobilitätsbarrieren begründen können. Das gleiche Argument hat später die Tendenz verstärkt, die Bevorzugung (Bias) in den Reservierungen abzubauen. Diese Entwicklung kann am Beispiel der Flugreservierungssysteme als mehrperiodiges Gefangenendilemma dargestellt werden (vgl. Abb. C.2.16). 


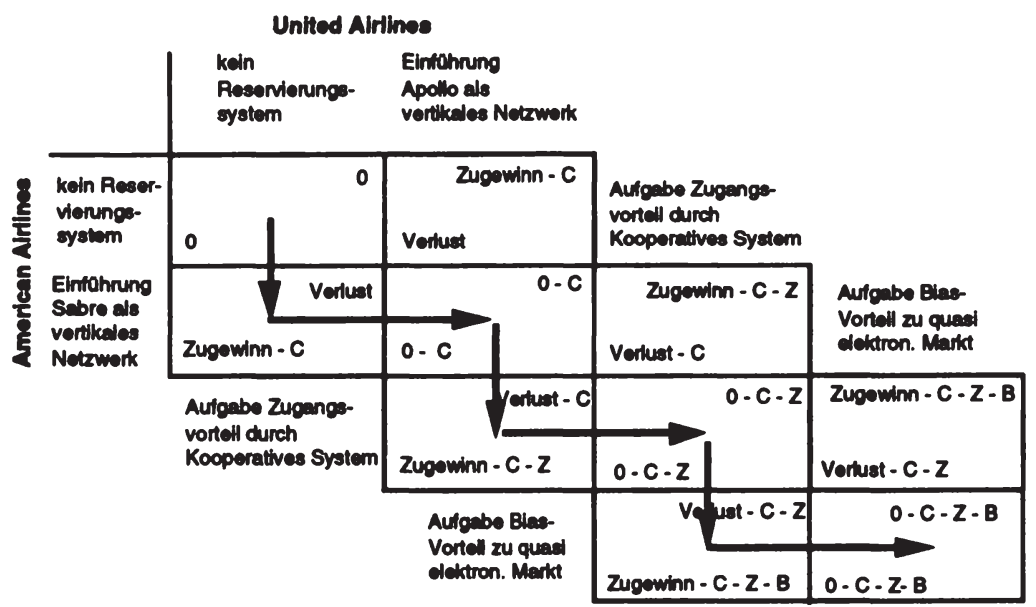

Abb. C.2.16: Degeneration vertikaler Netzworke zu quasl elektronischen Markten als mehrperlodges Gefengendilemma em Belsplel der Flugreservienungesysteme

In der ersten Runde wurde die Einführung von SABRE durch den Gegenzug der Einführung von Apollo neutralisiert. Sodann entschloß sich American aufgrund des drohenden Sortimentsvorteils von United (größerer Marktanteil, mehr Städtepaare), durch die Hereinnahme weiterer Fluggesellschaften seinen Zugangsvorteil $\mathrm{Z}$ aufzugeben, um mehr Reisebüros für SABRE zu gewinnen. United neutralisierte diesen Schritt durch eine entsprechende Strategie. Schließlich gab American, mit derselben Logik auch den Bias-Vorteil B auf. Die Flugangebote für ein Städtepaar umfassen oft mehrere Seiten im System, die Reservierung erfolgt jedoch in 70 - $90 \%$ aller Fälle aus der ersten Seite. Insofern hat der Bias, die Erstlistung der eigenen Flüge, erhebliche Auswirkungen auf die Wahl der Fluggesellschaft. Dies ist jedoch nicht im Interesse der Marktteilnehmer, hier der Reisebüros. Sie stehen ihrerseits im Wettbewerb mit anderen Reisebüros im Auffinden der günstigsten Angebote. Deshalb präferieren sie Systeme, die einen leichten (d.h. für sie personalkostengünstigen) Überblick über den gesamten Markt ermöglichen. Für den Marktteilnehmer ist ein kooperatives vertikales Netzwerk von höherem Nutzen, weil es zu einem quasielektronischen Markt umfunktioniert wird, der die focal Firm nicht begiinstigt.

Stehen die vertikalen Netzwerke zueinander im Wettbewerb, greift das Gefangenendilemma: Entfällt die Bevorzugung, kann die Zahl und Nut- 
zungsintensität der Marktteilnehmer erhöht werden. Führt dies jedoch zu Positionsvorteilen, wird der Bevorzugungsentfall zwangsläufig von anderen Netzwerken kopiert.

Der erhebliche Druck auf die Degeneration des vertikalen Netzwerks zum bevorzugungsfreien, quasielektronischen Markt belegt erneut die Instabilität vertikaler Netzwerke. American und United haben jeglichen Vorteil für den eigenen Geschäftsprozeß als Flugunternehmung verloren. Allerdings erzielen sie erhebliche Einnahmen als Marktträger. Die Marktträgerschaft ist in diesem Beispiel freilich so stark vom traditionellen Geschäft einer Fluggesellschaft abgetrennt, daß im Finalstadium eher von Diversifikation als von Integration gesprochen werden kann. Die Stabilität der Position von Marktträgern ist nunmehr zu analysieren.

\subsubsection{Dauerhaftigkeit der Wettbewerbsposition elektronischer Märkte}

Trotz der verschiedenen Positionsvorteile elektronischer Märkte gegenüber ihren Wettbewerbern, traditionellen Märkten und vertikalen Netzwerken, erreicht der Marktträger in vielen Fällen keine besonders hohe Rentabilität, verglichen mit dem oft erheblichen Handelsvolumen. Dies ist wie bei anderen Formen der Geschäftsnetzwerkintegration auf eine kaum zu gewährleistende Stabilität zurückzuführen. Wenn, wie häufig der Fall, der Träger eines elektronischen Marktes lediglich einen kopierbaren technologischen Vorsprung hat, wird dieser rasch imitiert werden, falls die Marktträgerschaft überhaupt profitabel ist. Aufgrund der Stabilitätsprobleme können die Marktteilnehmer die Effizienzvorteile des elektronischen Marktes abschöpfen. Eine stabile Position wird der Marktträger nur erreichen, wenn die Integrationsstrategie mit anderen Mobilitätsbarrieren verbunden werden kann.

Die einzige, wirklich greifende Mobilitätsbarriere ist die Marktgröße und die damit einhergehende Marktliquidität: Die Teilnahme an einem elektronischen Markt, sei es für Diamanten, Aktien, Automobile oder gebrauchte Bücher, ist für die Marktteilnehmer nur dann interessant, wenn ein großer Teil des Marktgeschehens in einem einzigen Markt erfaßt wird. Nur dann lohnt sich die spezifische Investition in etwaige für die Marktteilnahme erforderliche Hard- und Software und der Transaktionskosten verursachende Zeitaufwand für den Handel mit dem System - niemand will 
zehn Systeme parallel beobachten. Eine erfolgreiche Marktträgerschaft beruht auf erheblichen Skaleneffekten des Marktträgers, einerseits durch die Subadditivität seiner Kostenfunktion, andererseits durch das von den Marktteilnehmern gewünschte Mindestvolumen, um den Markt überhaupt interessant zu machen. Die Subadditivität der Kostenfunktion resultiert aus einem strategischen Commitment eines Netzbetreibers in das Netz. Ein strategisches Commitment liegt vor, wenn eine so hohe Bindungswirkung an starre Faktoren verursacht wurde, daß ein potentieller Wettbewerber vom Eintritt in den Markt absieht, weil er befürchten muß, daß der Netzbetreiber auch dann im Markt bleiben wird, wenn er (bei einmal eingegangenem Commitment) zu extrem geringen Grenzkosten anbieten muß. Damit wird ein potentieller Wettbewerber vom seinerseitigen Eingehen eines Kostenvorlaufs abgeschreckt. ${ }^{1}$ Im Beispiel von Aucnet hat CEO Fujisaki 1000 Händlerterminals auf Lager erworben, um ein strategisches Commitment einzugehen, obwohl ein Leasinggeschäft möglich gewesen wäre. ${ }^{2}$ Selbst ein kooperatives Netz der Auktionatorenvereinigung konnte sich gegen Aucnet nicht durchsetzen.

Der zweite wichtige Faktor, die Liquidität des elektronischen Marktes, kann oft allein durch die Größe des Netzes, also die Zahl der Marktteilnehmer, sichergestellt werden. Liegt aber dennoch ein strukturelles Ungleichgewicht vor, wird der Marktträger selbst versuchen müssen, die Liquidität zu erhöhen. MBS gelang dies durch eine innovative Kombination von relationalen Verträgen mit Universitätsbuchhandlungen und deren Angebot (knapper) gebrauchter Lehrbücher auf dem von MBS getragenen elektronischen Markt. Instinet gelang es durch die Zusammenarbeit mit Analystengruppen und Aktiengesellschaften, die an einem funktionierenden Sekundärmarkt interessiert waren, um in einzelnen Werten die Bedeutung traditioneller Börsen zu übertreffen. Ist diese Position erst einmal erreicht, wird sie kaum angreifbar sein. Denn die Existenz der Liquidität in einem elektronischen Markt verstärkt und stabilisiert dessen Bedeutung gegenüber "trockeneren" Handelsplätzen.

1 Zur Ökonomie der Netzwerke vgl. z.B. Gilbert, R.J., Mobility Barriers, a.a.O, S. 498 ff. Zu deren Bedeutung Albach, H., Eröffnungsansprache zur 52. Jahrestagung des Verbandes der Hochschullehrer für Betriebswirtschaftslehre, in: Betriebswirtschaftslehre und ökonomische Theorie, Jahrestagung des Verbandes der Hochschullehrer für Betriebswirtschaft vom 6. Juni 1990, (Hrsg.), Ordelheide, D., Rudolph, B., Büsselmann, E., Stuttgart, 1991 , S. 5.

2 Vgl. Warbelow, A., Kokuryo, J., Konsynski, B., Aucnet TV Auction Network System, a.a.O., S. 7. 
Die erheblichen Skaleneffekte begründen die Möglichkeit für einen "First Mover", hohe Mobilitätsbarrieren zu errichten. Das heute größte Flugreservierungssystem SABRE, getragen von American Airlines, ist zwar vom vertikalen Netzwerk zu einem quasielektronischen Markt degeneriert und bringt American weder Zugangs- noch Biasvorteile. ${ }^{1}$ Allerdings erzielt American Airlines als Marktträger mit diesem elektronischen Markt einen Jahresreingewinn von $\$ 300$ Mio., doppelt soviel wie im Fluggeschäft. Der CEO von Aucnet, Masataka Fujisaki, faßt plakativ zusammen: "In the Network Business, there is always the first mover, and never the second. ... Either you succeed big or fail big."2

3

Einsatz von KIS zur Integration von Geschäftsaktivitäten: Ergebnisse der Fallstudienuntersuchung

Die Transaktionsintegration, die Organisationsintegration, die Geschäftsprozeßintegration, die vertikalen Netzwerke, die Information Partnerships und die elektronischen Märkte sind sechs sehr verschiedene Integrationsstrategien. Sie unterscheiden sich im Hinblick auf die relevanten Dimensionen des vorgelegten Bezugsrahmens, insbesondere hinsichtlich der Kosten- und Nutzenwirkungen.

Die Ergebnisse der Fallstudienuntersuchung faßt Abbildung C.3.1 zusammen. Dabei ist jeweils die Anzahl der Fallstudien, die zur Entwicklung des Hauptarguments der jeweiligen Integrationsstrategie herangezogen wurde, angegeben. Durch die Kombination der drei ausführlich dargestellten Fallstudien mit den gleichfalls ausführlich analysierten, aber nur knapp skizzierten Fallstudien können die Ergebnisse

1 Es handelt sich nur um einen quasielektronischen Markt, weil AA immer noch als focal Firm Träger des vertikalen Netzes ist. Allerdings ist durch die Abschaffung der bevorzugten Listung, mit Ausnahme der Informationsgewinnung, jeglicher Vorteil für den Geschäftsprozeß von AA, das Fluggeschäft, aufgegeben worden.

2 Vgl. Warbelow, A., Kokuryo, J., Konsynski, B., Aucnet TV Auction Network System, a.a.O., S. 7. 


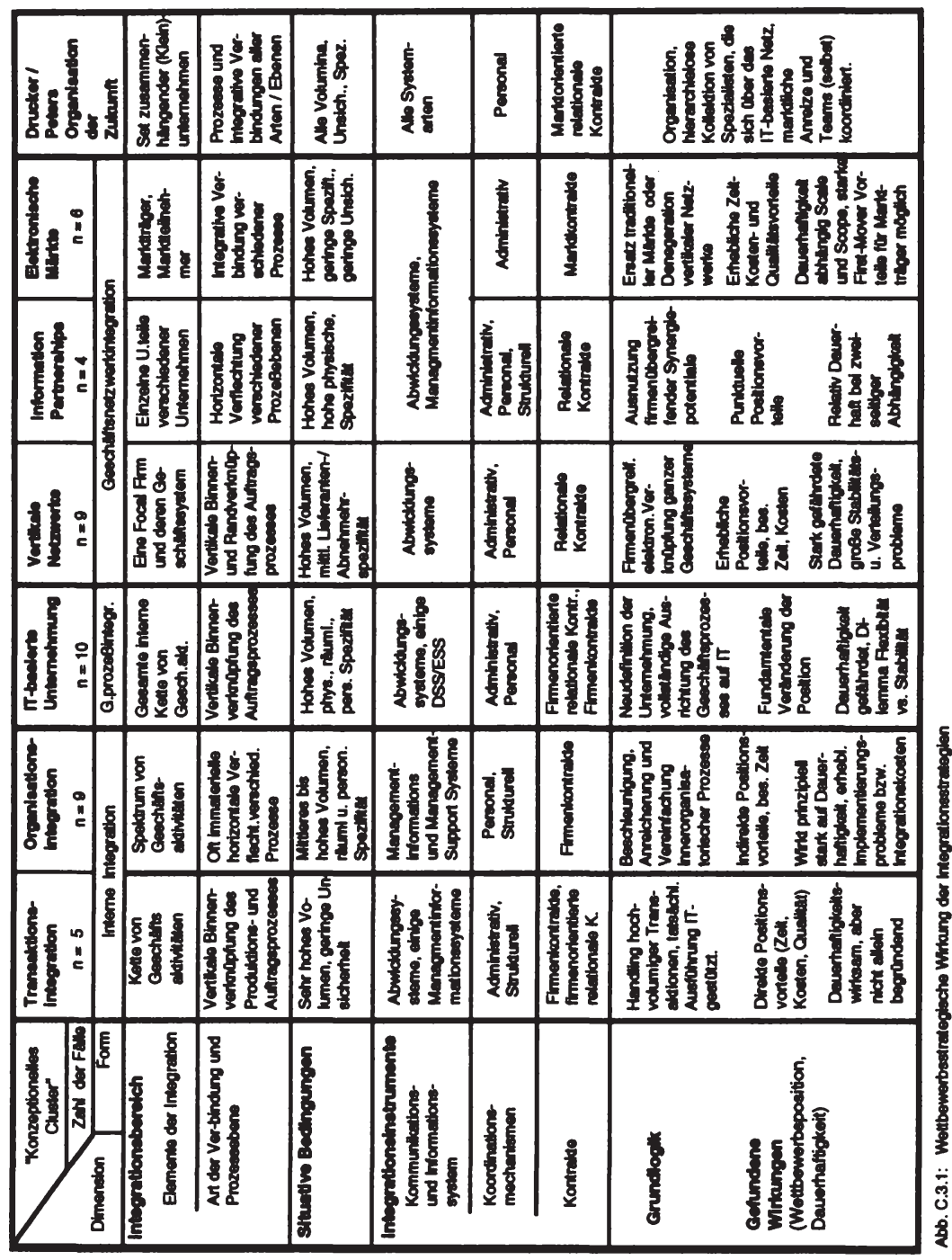

der explorativen Untersuchung immerhin eine interne Validität der Aussagen zu den einzelnen Integrationsstrategien beanspruchen.

Die intern orientierte Transaktionsintegration wird in vielen Auftragsprozessen eingesetzt, die gekennzeichnet sind von hohem Volumen bei relativ geringer Unsicherheit und Abwicklungsorientierung. Mit der Transaktions- 
integration können direkt Positionsvorteile erzielt werden, die allerdings relativ leicht kopierbar und selten dauerhaft sind. Dauerhafte Positionsvorteile werden nur in Abstimmung mit anderen Elementen der Wettbewerbsstrategie erreicht, die sowohl aus dem Grundgeschäft stammen können wie aus einer Kombination der Transaktionsintegration mit der Organisationsintegration.

Die Organistionsintegration wirkt indirekt auf die Wettbewerbsposition, etwa durch die Beschleunigung der Kommunikation, die Verbesserung der Genauigkeit von Informationen und Präsentationsmöglichkeiten, die Möglichkeit zu Ad-hoc-Analysen und zur Verbesserung der persönlichen Kommunikation und Teamkoordination. Weiterhin verändert sie das "mentale Modell" der Unternehmung durch breiteren Zugang zu rohen und unaggregierten Daten, zu externen Daten und durch Tests und Simulationen alternativer Annahmen und Vorgehensweisen. Der Einsatz von KIS beschleunigt den Managementprozeß. Kann darüber die organisatorische Trägheit reduziert und die operative Flexibilität erhöht werden, dürften die Positionsgewinne relativ stabil sein. Organisationale Integration ist aufgrund ihrer indirekten Wirkung nicht nur schwer meßbar, sondern auch von außen schwer beobachtbar - sie kann damit nicht leicht kopiert werden.

Die Geschäftsprozeßintegration hat eine erhebliche Wirkung auf die Wettbewerbsposition der dann KIS-basierten Unternehmung. Die vertikale Verknüpfung des Geschäftsprozesses zu einem störungsarmen, schnittstellenfreien Ablauf kann zu einer völligen Neudefinition des Geschäfts führen oder die Art des Geschäfts wesentlich verändern. In allen analysierten Beispielen ergaben sich jedoch erhebliche Stabilitätsprobleme. Zudem entstehen Flexibilitätsprobleme, wenn das der technischen Integration zugrundeliegende Strukturierungserfordernis der Geschäftsaktivitäten im Zeitablauf schwankt.

Ein vertikales Netzwerk, definiert durch elektronische vertikale Verknüpfungen zwischen einer "focal Firm" und deren Geschäftsnetzwerk, kann der focal Firm beträchtliche Positionsvorteile verschaffen, und zwar direkt durch Verkürzung der Abwicklungszeit, indirekt durch Verbesserung der Kosten- und Qualitätsposition. Jedoch kann die focal Firm des vertikalen Netzwerks die Stabilität der Wettbewerbsposition nicht allein durch Integration absichern. Vielmehr ist eine Kombination der Integrationsstrategie mit dem Grundgeschäft erforderlich, weil vertikale Netzwerke prinzipiell leicht 
imitierbar sind, einschließlich immer höherer (elektronischen) Funktionalität. Es bestehen intrikate Beziehungen zwischen Stabilität, Verteilung und Flexibilität. Die im Wettbewerb stehende Unternehmung kann dabei nicht wählen, ob Commitments in die elektronischen vertikalen Netzwerke vorgenommen werden sollen. Trotz mangelnder Stablisierbarkeit sind Investitionen in vertikale Netzwerke häufig allein notwendig, um Parität mit den Wettbewerbern herzustellen.

Auch im Falle von Information Partnerships sind dauerhafte Wettbewerbsvorteile nur möglich, wenn die Logik des Grundgeschäfts mit derjenigen der Integrationsstrategie gut übereinstimmt. Dabei ist die interne Stabilität einer Information Partnership tendenziell höher als die eines vertikalen Netzwerks, weil sie zwischen wenigen Unternehmen geschlossen werden, die möglicherweise sehr verschiedenen Geschäftsnetzwerken angehören, d.h. weder vertikal noch horizontal miteinander konkurrieren. Ein Druck auf die Information Partnerships kann daher nur von der Systemebene kommen, d.h. aus dem Wettbewerb der vertikalen Netzwerke oder der Information Partnerships untereinander.

Elektronische Märkte bilden sich entweder aus traditionellen Märkten oder aus vertikalen Netzwerken. Die Wettbewerbsposition eines Marktträgers hängt von der Zeit-, Qualitäts- und Kostenposition des elektronischen Marktes im Vergleich zu den alternativen Abwicklungsformen ab. Ist die Integrationsstrategie des Marktträgers allein auf Technologie aufgebaut, entstehen Stabilitätsprobleme, die zu einer Abschöpfung der Effizienzvorteile des elektronischen Marktes durch die Marktteilnehmer führen. Die einzige, wirklich greifende mögliche Mobilitätsbarriere ist die Marktgröße und -liquidität. Bei einer Subadditivität der Kostenfunktion wird das Eingehen eines echten strategischen Commitments in die Errichtung eines Netzes möglich, mit dem eine strukturelle Mobilitätsbarriere aufbaubar ist. Die Liquidität des elektronischen Marktes kann durch Eingreifen des Marktträgers in den Geschäftsprozeß selbst oder die Bildung relationaler Verträge mit Marktteilnehmern geschaffen werden.

Die zeitablaufbezogene Sicht bei der Analyse des KIS-Einsatzes zur Integration der Unternehmung ist entscheidend, denn die Schaffung eines Wettbewerbsvorteils ist sinnlos, wenn nicht die dazu eingegangenen Commitments zukünftige Renten erbringen, die eine Rückzahlung des Kostenvorlaufs ermöglichen. Dies gilt unabhängig davon, ob eine offensive Erhö- 
hung des rentenschöpfenden Erfolgspotentials angestrebt wird oder eine defensive Strategie zur Sicherung der Parität unter den Wettbewerbern.

Aufgrund der unstrittigen Wirkungen der KIS auf die Wettbewerbsposition haben sich einige Branchen bereits grundlegend verändert und viele weitere sind im Begriff dazu. Dabei stehen die verschiedensten Stufen des Geschäftssystems in einem komplexen kooperativ-kompetitiven Verhältnis zueinander. Die Unternehmung ist Teil eines Geschäftsnetzwerks und dem Druck des Wettbewerbs des eigenen mit anderen Geschäftsnetzwerken ausgesetzt. Sie kann darüber hinaus mit Unternehmen, die in ähnlichen Geschäftsfeldern operieren, um die Stabilität ihrer Position ringen. Zusätzlich steht sie mit den vor- und nachgelagerten Stufen innerhalb des Geschäftsnetzwerks in einem vertikalen Wettbewerb um die Verteilung des (gemeinsam) geschöpften Werts. Gleichzeitig stehen jene vor- und nachgelagerten Stufen des Geschäftsnetzwerks ihrerseits im Wettbewerb mit Unternehmen vergleichbarer Geschäftsfelder. Angesichts der potentiellen Komplexität dieser Zusammenhänge dürfte es problematisch sein, eine hinreichend präzise Bezugsgröße für das Konstrukt "Wettbewerbsvorteil" zu definieren, das sich ja immer nur aus dem Vergleich bestimmen läßt. Es wird nichts anderes übrig bleiben, als die Analyse jeweils auf das Geschäftsnetzwerk (sowohl auf vertikale Netzwerke als auch Information Partnerships), die eigene Unternehmung und die vor- und nachgelagerten Unternehmungen zu beziehen. Integrationsstrategien nach "neuen Regeln" werden dabei häufig am gesamten Geschäftsnetzwerk ansetzen und auf einer neuen Dekomposition und Integration dieser Ebenen aufbauen, wie zum Beispiel anhand des inhärenten Drucks zur Entwicklung der isolierten Unternehmung des vertikalen Netzwerks, und vom vertikalen Netzwerk zum elektronischen Markt aufgezeigt wurde.

Eine Extrapolation der gefundenen Tendenz neuer Integrationstrategien mit KIS, die zugegebenermaßen spekulativ und nicht durch die Untersuchung gedeckt ist, würde eine Entwicklung zur "Organisation der Zukunft" bestätigen. Diese wird häufig beschrieben als Set eng verflochtener, relativ kleiner, fokussierter Unternehmen, die sich durch marktorientierte Kontrakte, starken KIS-Einsatz und personale Koordinationsmechanismen koordinieren und Vorteile von Fokus und Skaleneffekten kombinieren. ${ }^{1}$ Die Größe

1 Vgl. Drucker, F. New Organization, in: a.a.O., S. 45 ff.; Peters, T., Destruction of Hierarchy, a.a.O., S. 33 ff. 
dieser "kleinen" Unternehmung wird dabei mehr auf die Tiefe des Geschäftsprozesses bezogen, den sie unmittelbar kontrolliert, keineswegs z.B. auf die erforderliche Kapitalbasis. So ist die Marktträgerschaft eine interessante Rolle in einem elektronischen Markt, kann aber ganz erhebliche (strategische) Commitments zum Aufbau von Netzinfrastrukturen erfordern. Dafür wird u. a. die Kreation innovativer relationaler Verträge in der Finanzierung erforderlich sein. Das notwendige Ausmaß und die Möglichkeit strategischer Commitments zum Aufbau von Infrastrukturen wird seinerseits von sich entwickelnden Standards abhängen, die die Starrheit und Spezifität elektronischer Infrastrukturen reduzieren und die Tendenz zu eher marktorientierten Kontrakten verstärken werden. 


\section{Zusammenfassung}

Niccolo Machiavelli wies Lorenzo de' Medici darauf hin, daß eine leicht errungene Position auch leicht verloren geht. Dies gilt auch für den Einsatz von KIS zur strategischen Integration von Geschäftsaktivitäten, von dem keine einfache, aber stabile Veränderung der Wettbewerbsposition erwartet werden kann, wie dies in der Literatur gelegentlich suggeriert wird. Bei allen Integrationsstrategien ließ sich feststellen, daß in der Tat erhebliche Positionsvorteile erreicht werden können. Diese sind jedoch inhärent instabil, und zwar um so instabiler je größer sie sind. Gleichzeitig wird es an der als Gefangenendilemma formulierten kompetitiven Interdependenz deutlich, daß gewissermaßen ein Zwang zum Einsatz von KIS zur strategischen Integration immer dann besteht, wenn der Einsatz möglich erscheint. Zur Bestimmung der Möglichkeit des KIS-Einsatzes zur Integration ist der Integrationsbereich genau zu definieren und ein Mapping vorzunehmen. Dabei sind die Schnittstellen und Gleichartigkeiten zwischen den Unternehmensteilen bzw. den Geschäftsaktivitäten, die sie tragen, zu identifizieren. Unter Einsatz der drei Integrationsinstrumente, KIS, Koordinationsmechanismen und Kontrakte, können Schnittstellenverluste abgebaut und Gleichartigkeiten ausgenutzt werden, indem vertikale Verknüpfungen und horizontale Verflechtungen zwischen den Unternehmensteilen gebildet werden. Kriterium zur Beurteilung, ob solche integrativen Verbindungen vorteilhaft sind oder nicht, ist deren Beitrag zum dauerhaften Wettbewerbsvorteil.

Basis des dauerhaften Wettbewerbsvorteils der Unternehmung sind Commitments in ein partiell fixes Faktorbündel. Das strategische Problem der Unternehmung ist die Maximierung von Renten aus diesem partiell fixen Faktorbündel und die Komposition desselben. Aus der Fixierung des Faktorbündels erwächst der dynamische, zeitablaufbezogene Charakter des strategischen Problems. Nicht nur die Wettbewerbsposition als Produkt der Qualität, Kosten und Zeitposition ist zu betrachten, sondern besonders deren Dauerhaftigkeit als Produkt der Stabilität, Flexibilität und Verteilung. 
strategischen Ansätzen. Erstens wird gegenüber der statischen bzw. komparativ-statischen Perspektive, die nur die Kosten- vs. Differenzierungsvorteile betrachtete, eine dynamische, d.h. zeitablaufbezogene Perspektive eingenommen. Damit werden explizit die Bindungswirkungen sowohl auf die Position als auch auf deren Dauerhaftigkeit untersucht. Zweitens ermöglicht es die Definition des Erfolgspotentials als das Potential zur Erzielung von Rente aus einem partiell fixen Faktorbündel, das komplexe Phänomen des Wettbewerbs zwischen mehreren Stufen im Geschäftssystem abzubilden. Die Definition des traditionellen Wettbewerbsvorteils erfolgt ausschließlich auf der Ebene von Produkt-/Marktkombinationen, d.h. auf SBU-Ebene. ${ }^{1}$ Zur Betrachtung des strategischen Integrationsproblems war es jedoch erforderlich, den horizontalen wie vertikalen Wettbewerb innerhalb und zwischen allen Stufen des Geschäftssystems, zwischen Netzwerken oder zwischen Geschäftssystemen zu erfassen, und nicht lediglich den horizontalen Wettbewerb in einem Geschäftsfeld. Dazu erwies sich die Unterscheidung von Position und Dauerhaftigkeit und innerhalb der Dauerhaftigkeit der Stabilität, der Verteilung und der Flexibilität als relativ robust, um sehr verschiedene wettbewerbsstrategische Wirkungen von Integrationsstrategien abzubilden. Drittens ermöglicht das Mapping des Integrationsbereichs und die Darstellung einzelner Prozesse im Vergleich zur Wertkettendarstellung eine dem Integrationsproblem gemäßere Dekomposition der Unternehmung in ihre Geschäftsaktivitäten. Das Mapping kann als Werkzeug eines Integrationsmanagements dienen. Explizit werden die Geschäftsaktivitäten von den sie tragenden Unternehmensteilen unterschieden, damit genau herausgearbeitet werden kann, was eigentlich verknüpft oder verflochten werden soll. Zugleich wird die Vermengung von Zuständigkeit und Tätigkeit vermieden. Die Einbeziehung der Zeit bei der Definition des Integrationsbereichs eignet sich zudem, betriebliche Prozesse operational darzustellen. Es werden Erkenntnisse einbezogen, die in der aggregierenden Betrachtung von Kosten- oder Qualitätsinformationen untergehen mögen.

1 Porter, M.E., Competitive Advantage, a.a.O., S. 36. 
In der explorativen Untersuchung wurden einige Ausnahmen gefunden, für die der eingangs erwähnte Dauerhaftigkeitspessimismus gegenüber Positionsvorteilen, die durch KIS-gestützte Integration geschaffen wurden, nicht zutrifft. Diese Ausnahmen haben als gemeinsamen Nenner eine notwendige Grundbedingung und zwei alternative hinreichende Bedingungen. Die notwendige Grundbedingung ist eine sehr enge Ausrichtung des Grundgeschäfts und der Integrationsstrategie aufeinander, d.h. die Schnittstellen und Gleichartigkeiten des Geschäftsprozesses müssen der Strukturierungsanforderung des KIS-Einsatzes entsprechen oder entsprechend angepaßt werden können.

Eine erste hinreichende Bedingung zur Sicherung dauerhafter Positionsvorteile liegt vor, wenn der durch strategische Integration mit KIS geschaffene Positionsvorteil kombiniert werden kann mit Dauerhaftigkeitsvorteilen außerhalb der Integrationsstrategie. Die wichtigsten solcher Dauerhaftigkeitsvorteile sind die Marktführerschaft in einem von Skalen- und Scopeeffekten geprägten Geschäft, nicht kopierbare Qualitätsvorteile sowie Zeitvorteile aus geringerer organisationaler Trägheit, die die Imitation verzögern. Alternativ dazu kann die Dauerhaftigkeit gesichert werden, wenn die Unternehmung mit der Integrationsstrategie ein strategisches Commitment eingeht, d.h. wenn ein so hoher Kostenvorlauf in Kauf genommen wird, daß eine Imitation durch die Wettbewerber unwahrscheinlich wird, die den Kostenvorlauf noch nicht eingegangen sind. Dies wird bei der Errichtung von Netzinfrastrukturen für elektronische Märkte häufig der Fall sein. Hier kann der Marktträger angesichts subadditiver Kostenfunktionen und der Liquiditätsanforderung der Marktteilnehmer an den Markt quasi natürliche Monopole schaffen.

Aber auch diese beiden einzigen Dauerhaftigkeitsstrategien sind nicht ohne Risiken. Erstens zieht ein Eingehen strategischer Commitments nicht nur Stabilitätsvorteile, sondern auch Bindungsnachteile nach sich. Die Stabilitätsvorteile beugen zwar wirksam der Imitation durch Wettbewerber vor, jedoch nicht deren Untergrabung durch mögliche neue Regeln. Eine aus 
Sicht des Marktführers besonders gefährliche Defensivstrategie der Wettbewerber ist deren Kooperation in einem System. Damit dividiert sich das Commitment der Wettbewerber durch die Zahl der Teilnehmer, während sich die Wirkung des Netzes addiert. Zweitens wird, sobald der Wettbewerbsvorteil wirklich dauerhaft, sichtbar und meßbar ist und den Charakter eines natürlichen Monopols anzunehmen droht, ein administrativer Eingriff durch die Wettbewerbsbehörden erfolgen. So stehen die Betreiber der Flugreservierungssysteme im Rechtsstreit mit Marktteilnehmern, insbesondere über die Höhe der Nutzungsbeiträge und das Recht der Marktträger, Informationen aus dem Reservierungssystem für ihre Geschäftspolitik zu nutzen. Alle Beispiele einer erfolgreichen Integrationsstrategie machten große Wettbewerber größer, stabile Vorteile stabiler. Aus Sicht der Wettbewerbspolitik liegt hier ein schwieriges Dilemma vor. Einerseits wird es nicht effizient sein, viele elektronische Märkte nebeneinander zu betreiben. Andererseits besteht die Frage, ob nicht erhebliche Marktzugangsbeschränkungen vorliegen und die Betreiber asymmetrisch Nutzen aus dem System ziehen können. Auch kooperative Netze eines Branchenverbands sind keine Patentlösung. Hier müssen Änderungen und die Fortentwicklung des Netzwerks, die im Sinne der Marktteilnehmer wären, in einem schwierigen "politischen Prozeß" diskutiert werden, so daß sie eventuell unterbleiben, das System einer raschen Obsoleszenz unterliegt und hinter den Möglichkeiten zurückbleibt.

Neben diesen wettbewerbspolitischen Problemen wirft der Einsatz von KIS zur Integration von Geschäftsaktivitäten eine Fülle weiterer Fragen jenseits der in dieser Arbeit analysierten Wirkung auf den dauerhaften Wettbewerbsvorteil auf. Spätestens seit Ackoffs Beitrag "Management Misinformation Systems"1 übt die verhaltenswissenschaftlich orientierte Organisationstheorie Kritik am Einsatz von Informationstechnologie. Mit der Abkehr von der zentralen Kontrollorientierung der KIS und der Betonung einer dezentralen Motivationsorientierung wird diese Kritik zwar schwächer,

1 Ackoff, L., Misinformation Systems, a.a.O., S. B147 - B156. 
jedoch keineswegs verstummen. Die Wirkungen einer weitgehenden Substitution etwa der Strukturorganisation durch das KIS auf die vier "weichen S" müssen als ungewiß bezeichnet werden. ${ }^{1}$ Walton weist auf die "duale Potentialität" der Informationstechnologie hin, sowohl kontrollorientiert als auch motivationsorientiert ausgelegt zu werden. Er zeigt an vielen Fällen, daß und wie entscheidend es darauf ankommt, bei der Gestaltung und Implementierung des KIS die Motivationsorientierung zu betonen. ${ }^{2}$

Im Zusammenhang mit der Implementierung bei der Einführung von KIS weist Applegate darauf hin, daß die erfolgreichsten Einsatzbeispiele dort zu finden sind, wo zuvor noch kein traditionelles "EDV"-System vorhanden war, so daß eine völlig neue Architektur möglich wurde, z.B. bei Frito Lay. ${ }^{3}$ Demgegenüber fielen gerade den Banken und Versicherungen, die frühesten Nutzer der Informationstechnologie, selbst trivial erscheinende Integrationsbemühungen sehr schwer. Beispielsweise benötigten viele US-Banken mehrere Jahre, um in Verbindung mit der Einführung eines täglich verzinslichen Money-Market-Kontos einen integrierten Kontoauszug für alle Anlage- und Kreditformen einzuführen. Auch bei Schnellrestaurants waren die wesentlichen Integrationshindernisse die existierenden POSTerminals. ${ }^{4}$ Banken sind Commitments in EDV-Anlagen alten Stils eingegangen, und diese Commitments haben Bindungswirkung: Die Anlagen sind partiell starr, Optionen für eine Rearchitektur entgehen und dies nicht zuletzt bei erheblicher prozessualer und organisationaler Trägheit. Das daraus sich ergebende Implementierungsparadox trifft genau die "informationsintensiven" Unternehmen: Als die frühesten Nutzer von KIS haben sie sich zeitweilig selbst an Systeme gebunden, die nicht mehr der heute möglichen Architektur entsprechen - das traditionelle S-Kurven-

1 Structure, Strategy und Systems werden oft als harte S, Shared Values, Skills, Style und Staff als weiche $S$ im $7 \mathrm{~S}$-Ansatz genannt. Vgl. Peters, T., Waterman, J., In Search of Excellence, a.a.O., S. 8 - 19.

2 Vgl. Walton, R.E., Up and Running, a.a.O., bes. S. 26 ff.; vgl. auch Kubicek, H., Informationstechnologie, a.a.O., S. 236 ff.

3 Vgl. Main, T., Computers, a.a.O., S. 121; Simson, C. von, Food Fight, a.a.O., S. 24. 
Problem des Technologiemanagements. ${ }^{1}$ Angesichts dieses Befundes wird erneut deutlich, daß die Umstellung des gesamten Geschäftsprozesses auf KIS-gestützte Integration sich nachteilig auf die strategische Flexibilität auswirken kann.

Sollten KIS-gestützte Integrationsstrategien strukturelle Koordinationsmechanismen und unternehmenskonstituierende Kontrakte so aufweichen, daß sie durch Netzwerke oder quasielektronische Märkte abgelöst werden, erhebt sich die Frage, inwieweit eine auf Unternehmenskultur aufbauende Integration noch möglich ist. Gemeinsame Grundwerte oder die Kultur einer Organisation sind heute oft eng mit der Grenze unternehmenskonstituierender Kontrakte verknüpft und von der reichen, informalen Kommunikation innerhalb einer Hierarchie mit bedingt. Es ist fraglich, wie diese für die Integration der Unternehmung wohl sehr wichtige Kommunikation über ein Netzwerk oder gar einen (quasi-) elektronischen Markt hinweg möglich ist. Netzwerke weisen eine stärkere Gemeinsamkeit mit demokratischen, politischen Entitäten auf als mit hierarchischen Strukturen, mögen insofern informale Koordinationsmechanismen entwickeln, die eher aus dem politischen Leben als aus dem Wirtschaftsleben bekannt sind. Eine weitere sich hier ergebende Frage ist die Bedingtheit von Integration und nationaler Identität bzw. gesellschaftlichem Kontext. Beispielsweise kann eine kontraktuelle Deintegration eher in Gesellschaften möglich sein, in denen Divergenzen der Zielfunktion nur begrenzt zu opportunistischer Abschöpfung führen, wie dies etwa Japan zugesprochen wird. Schließlich wird sich bei stärkerem Einsatz von KIS die Frage der Datensicherheit und des Datenschutzes immer wieder erheben angesichts der immer weitergehenden und breiter distribuierten Verfügbarkeit von Informationen.

Es liegen kaum veröffentlichte Studien über gescheiterten oder nicht erfolgreichen Einsatz von KIS zur Integration von Geschäftsaktivitäten vor. Die-

4 Vgl. Simson, C. von, Food Fight, a.a.O., S. 24. 
ses Defizit wurde auch in dieser Arbeit mangels Daten nicht wesentlich verringert. In der theoretischen und empirischen Untersuchung nicht erfolgreichen KIS-Einsatzes liegen noch lohnende Potentiale für die Forschung, auch als "Kontrollgruppe" jeglicher Ansätze, die sich auf erfolgreiche Anwendungen konzentriert haben.

Der Einsatz von Informationstechnologie hat erst begonnen, die Unternehmung und ihre Position im Wettbewerb zu verändern. Es wird nachhaltige Forschungsbemühungen erfordern, den einsetzenden Substitutionsprozeß unter den Integrationsinstrumenten weiter $\mathrm{zu}$ verfolgen, einschließlich einer die Praxis eng begleitenden Untersuchung des Implementierungsproblems.

1 Vgl. Foster, R., Innovation, New York, 1986, S. 87 - 111. 


\section{Literaturverzeichnis}

Aaker, D. A., Kriterien zur Identifikation dauerhafter Wettbewerbsvorteile, in: Wettbewerbsvorteile und Wettbewerbsfähigkeit, Hrsg.: Simon, H., Stuttgart, 1988.

Abell, D. F., Defining the Business. The Starting Point of Strategic Planning, Englewood Cliffs, N.J., 1980.

Adam, D., Kurzlehrbuch Planung, Wiesbaden 1986.

Adam, D., Entscheidungsorientierte Kostenbewertung, Wiesbaden, 1970.

Adam, D., Koordinationsprobleme bei dezentralen Entscheidungen, in: Zeitschrift für Betriebswirtschaft, 39. Jg., Oktober 1969, S. 615 - 632.

Adam, D., Backhaus, K., Meffert, H., Wagner, H., Hrsg., Integration und Flexibilität. Eine Herausforderung für die Allgemeine Betriebswirtschaftslehre, Wiesbaden, 1989.

Akerlof, G.A., The Market for Lemons: Quality Uncertainty and the Market Mechanism, in: Quarterly Journal of Economics, Vol. 84, 1970, S. 488 - 500.

Albach, H., Eröffnungsansprache zur 52. Jahrestagung des Verbandes der Hochschullehrer für Betriebswirtschaftslehre, in: Betriebswirtschaftslehre und ökonomische Theorie, Jahrestagung des Verbandes der Hochschullehrer für Betriebswirtschaft vom 6. Juni 1990, Hrsg.: Ordelheide, D., Rudolph, B., Büsselmann, E., Stuttgart, 1991, S. 5.

Alchian, A., Uncertainty, Evolution, and Economic Theory, in: Journal of Political Economy, Vol. 58, 1950, S. 211 - 221.

Alchian, A.A., Demsetz, H., Production, Information Costs and Economic Organisation, in: American Economic Review, Vol. 62, 1972, S. 777 795.

Allaire, Y., Firsirotu, M. E., Coping with Strategic Uncertainty, in: Sloan Management Review, Vol. 31, Spring 1989, S. 7 - 16.

Anthony, R.N., Planning and Control Systems: A Framework for Analysis, Cambridge: Harvard University Graduate School of Business Administration, Studies in Management Control, 1965.

Antonelli, C., Dall'economia industriale all'organizzazione industriale, in: Economia Politica, Vol. 2, 1987, S. 277 - 320. 
Applegate, L. M., Cash, J. I., GE Canada: Designing a New Organization, Boston, Massachusetts, Harvard Business School Case No. 9-189-138, Rev. 11/89, 1989.

Applegate, L. M., Cash, J. I., Mills, D. Q., Information Technology and Tomorrow's Manager, in: Harvard Business Review, Vol. 66, November - December, 1988, S. 128 - 136.

Applegate, L.M., Hertenstein, J.H., Wishart, N., Adonizio, M., Westinghouse Electric Corp.: Automating the Capital Budget Process (A), mit Supplements 1-3, Boston, Massachusetts, Harvard Business School Case No. 9-189-119 - 9-189-122, 1989.

Applegate, L. M., Wishart, N., Frito Lay, Inc.: A Strategic Transition (C), Boston, Massachusetts, Harvard Business School, Case No. N9-190$071,1990$.

Argyris, Ch., Overcoming Organizational Defenses, Needham Heights, 1990.

Argyris, Ch., Integrating the Individual and the Organization, New York, N. Y., 1964.

Argyris, C., Putnam, R., McLain Smith, D., Action Science, San Francisco, London 1985.

Arkes, H. R., Blumer, C., The Psychology of Sunk Cost, in: Organization Behavior and Human Decision Processes, Vol. 35, 1985, S. 124 140.

Arrow, K.J., The Economics of Agency, in: Principals and Agents: The Structure of Business, Hrsg.: Pratt, J.W., Zeckhauser, R.J., Boston, Massachusetts, 1985, S. 37 - 51.

Arrow, K. J., 1964 Optimal Capital Policy, the Cost of Capital, and Myopic Decision Rules, Annals of the Institute of Statistics and Mathematics, Tokyo, Vol. 16, S. 21 - 30.

Awad, E.M., Management Information Systems, Concepts, Structure and Applications, California, 1988.

Axelrod, R., The Evolution of Cooperation, New York, N. Y., 1984.

Bain, J. S., Industrial Organization, 2nd ed., New York, N. Y., 1968.

Bain, J. S., Barriers to New Competition, Cambridge, Massachusetts, 1956.

Bakos, Y.J., Interorganizational Information Systems: Strategic Implications for Competition and Coordination, Unpublished Doctoral Dissertation, Massachusetts Institute of Technology, Cambridge, Massachusetts, 1987. 
Balassa, B., The theory of economic integration, Homewood, Ill., 1961.

Ballwieser, W., Berger, K. H., Hrsg., Information und Wirtschaftlichkeit, Wiesbaden, 1985.

Barnard, Ch., The Functions of the Executive, Neudruck, Cambridge, Massachusetts, 1938.

Barney, J.B., Ouchi, W.G., Organizational Economics, San Francisco, London, 1986.

Barrett, S., Konsynski, B., Inter-Organizational Information Sharing Systems, in: MS Quarterly, Vol. 8, 1982, S. 93 - 105.

Barzel, Y., Measurement Cost and the Organization of Markets, in: Journal of Law and Economics, Vol. 25, 1982, S. 27 - 48.

Baxter Healthcare Corporation, Annual Report to Shareholders, February 20, 1989, S. 20.

Beck, L., Frito Lay Takes a Fresh Approach to Automation, in: Modern Materials Handling, Vol. 42, No. 1, 1987, S. 78 - 81.

Bell, D. E., Disappointment in Decision Making under Uncertainty, in: Operations Research, Vol. 33, 1985, S. 1 - 27.

Bell, D. E., Regret in Decision Making under Uncertainty, in: Operations Research, Vol. 30, 1982, S. 961 - 981.

Benjamin, R.I., DeLong, D.W., Scott Morton, M.S., The Realities of Electronic Data Interchange: How Much Competitive Advantage?, Working Paper No. 166, Massachusetts Institute of Technology, Center for Information Systems Research, Cambridge, Massachusetts, 1988.

Benjamin, R. I., Scott Morton, M. S., Information Technology, Integration, and Organizational Change, Working Paper No. 138, Massachusetts Institute of Technology, Center for Information Systems Research, Cambridge, Massachusetts, 1986.

Benkenstein, M., F\&E und Marketing, Bd. 20 der Schriftenreihe Unternehmensführung und Marketing, Hrsg.: Meffert, H., Steffenhagen, H. Freter, H., Wiesbaden 1987

Benkenstein, M., Henke, N., Vertikale Integration - Eine transaktionskostentheoretische Interpretation, Arbeitspapier Nr. 39 des Instituts für Marketing, Münster, 1990. 
Blackburn, J. D., New Product Development: The New Time Wars, in: Time-Based Competition, Hrsg.: Blackburn, J. D., Homewood, Illinois, 1991.

Blackburn, J. D., Hrsg., Time-Based Competition, Homewood, Illinois, 1991.

Blois, K.J. Vertical Quasi-Integration, in: The Journal of Industrial Economics, Vol. 20, S. 253 - 272.

Bodurtha, St., G., Perold, A.F., Instinet Corporation (A), Boston, Massachusetts, Harvard Business School Case No. 1-286-116, Revised Version, Boston 1988.

Bond, C., ATMs Pay Out, but Do They Pay Off?, in: Bankers Monthly, Vol. 106, No. 12, December 1989, S. 40 - 42.

Boynton, A. C., Shank, M. E., Zmud, R. W., Critical Success Factor Analysis as a Methodology for MIS Planning, in: MIS Quarterly, Vol. 9, 1985.

Brady, R.H., Computer in Top-Level Decision Making, in: Harvard Business Review, Vol. 45, July-August 1967, S. 67 - 76.

Brenner, W., Hilbers, K., Österle, H., "State of the Art" des Informationssystem-Managements, Arbeitsbericht Nr. IM 2000/CCIM 2000/4, Version 1.0, Hochschule St. Gallen, Institut für Wirtschaftsinformatik, 1990.

Brown, B., Bank Hones Its Strategic Edge, in: Network World, Vol. 5, No. 5, February 1, 1988, S. 1 und 46.

Buaron, R., New-Game Strategies, in: The McKinsey Quarterly, Spring 1981, S. 24 - 40.

Bullen, Ch.V., Johansen, R., Groupware: A Key to Managing Business Teams, Working Paper No. 138, Massachusetts Institute of Technology, Center for Information Systems Research, Cambridge, Massachusetts, 1988.

Bullen, Ch. V., Rockart, J. F., A Primer On Critical Success Factors, Working Paper No. 69, Center for Information Systems Research, Sloan School of Management, MIT, Cambridge, Massachusetts, June 1981.

Burns, T., Stalker, G. M., The management of innovation, London, 1961.

Busch, E. W., Integrationskonzepte der Betriebswirtschaftslehre: das Beispiel der Harvard Business School, in: Die Betriebswirtschaft, Vol. 50, S. 237 - 248. 
Buzzell, R. D., Gale, B. T., Das PIMS/Programm. Strategien und Unternehmenserfolg, Wiesbaden, 1989.

Caves, R. E., Economic Analysis and the Quest for Competitive Advantage, in: American Economic Review, Vol. 74, 1984, S. 127 - 132.

Caves, R., Porter, M. E., Barriers to Exit, in: Essays in Industrial Organization in Honor of Joe S. Bain, Hrsg.: Masson, R., Qualls, P., Cambridge, Massachusetts, 1976, S. 3- 34.

Cecil, J., Goldstein, M., Sustaining competitive advantage from IT, in: McKinsey Quarterly, 1990, S. 74 - 89.

Chandler, A. D., Strategy and Structure: Chapters in the History of the American Enterprise, Cambridge, Massachusetts, London, England, 1962.

Chess, D. M., Colishaw, M. F., A Large-Scale Computer Conferencing System, in: IBM Systems Journal, Vol. 26, No. 1, 1987, S. 138 - 153.

Child, J., Organizational Structure, Environment, and Performance: The Role of Strategic Choice, in: Sociology, Vol. 5, 1972, S. 1 - 22.

Ciborra, C., Reframing the Role of Computers in Organizations - The Transactions Costs Approach, in: Office: Technology and People, Vol. 3, 1987, S. 17-38.

Clarke, R. F., The Application of Information Technology in an Investment Management Firm, Unpublished Thesis, Massachusetts Institute of Technology, Sloan School of Management, Cambridge, Massachusetts, 1985.

Clemons, E. K., Row, M., McKesson Drug Company: A Case Study of Economost - A Strategic Information System, in: Journal of Management Information Systems, Vol. 5, Summer 1988, S. 36 - 50.

Clippinger, J. H., Konsynski, B. R., Information Refineries, Author's Draft, to be published in: Computerworld, August 1989, S. 73 - 77.

Coase, R., On the Nature of the Firm, in: Economica, Vol. 4, 1937, S. 331 351.

Cohen, J. E., An Uncertainty Principle in Demography and the Unisex Issue, in: The American Statistician, Vol. 40, February 1986, S. 32 - 39.

Cooper, R., Kaplan, R. S., Measure Cost Right: Make the Right Decisions, in: Harvard Business Review, Vol. 66, No. 5, 1988, S. 96 - 103.

Copeland, C.G., McKenney, J.L. Airline Reservations Systems: Lessons from History, in: MS Quarterly, Vol. 12, September 1988, S. 353-370. 
Copeland, T., Koller, T., Murrin, J., Valuation. Measuring and Managing the Value of Companies, New York, N. Y., 1990.

Costello, B., Systems Integration, in: Computing for Business, Vol. 10, Iss. 6,1985, S. $63-65$.

Coyne, K. P., Die Struktur dauerhafter Wettbewerbsvorteile, in: Wettbewerbsvorteile und Wettbewerbsfähigkeit, Hrsg.: Simon, H., Stuttgart, 1988, S. 18 - 29.

Cremer, J., Cooperation in Ongoing Organizations, in: Quarterly Journal of Economics, Vol. 101, 1986, S. 33 - 50.

Crutchfield, R.J., Applications: Getting a Leg Up by Using Handhelds, in: Datamation, Vol. 33, No. 1, 1987, S. 32 - 34.

Cyert, R. M., March, J. G., A Behavioral Theory of the Firm, Englewood Cliffs, N.J., 1963.

Cyert, R. M., March, J. G., Organizational Factors in the Theory of Oligopoly, in: Quarterly Journal of Economics, Vol. 70, 1956, S. 44-64.

Daniel, R. D., Management Information Crisis, in: Harvard Business Review, Vol. 39, September/October 1961, S. 111 - 121.

Dannenberg, J., Mikrocomputergestützte Instrumente der strategischen Unternehmensplanung, Wiesbaden, 1990.

Day, M., The Art of Systems Integration, in PEM: Plant Engineering \& Maintenance (Canada), Vol. 13, Iss. 2, 1990, S. 40 - 44.

Delfmann, W., Das Netzwerkprinzip als Grundlage integrierter Unternehmensführung, in: Der Integrationsgedanke in der Betriebswirtschaftslehre: Helmut Koch zum 70. Geburtstag, Hrsg.: Delfmann, W., Kuhn, Alfred, Wiesbaden, 1989, S. 87 - 113.

Delfmann, W., Kuhn, A., Hrsg., Der Integrationsgedanke in der Betriebswirtschaftslehre: Helmut Koch zum 70. Geburtstag, Wiesbaden, 1989.

DeSanctis, G., Gallupe, R.B., A Foundation for the Study of Group Decision Support Systems, in: Management Science, Vol. 33, 1987.

DeSanctis, G., Gallupe, R.B., Group Decision Support Systems: A New Frontier, Data Base, Winter 1985.

Dhebar, A.S., American Hospital Supply Corporation: The ASAP System, Boston, Massachusetts, Harvard Business School Teaching Note No. 9-187-014, Rev. 02/88, 1988. 
Dhebar, A.S., Warbelow, A., Ostofsky, K., Batterymarch Financial Management: Information Systems and Technology, Harvard Business School Case No. 9-188-013, Boston 1987.

Dixit, A., The Role of Investment in Entry Deterrence, Economic Journal, Vol. 90, 1981, S. 95 - 106.

Drucker, P. F., The Coming of the New Organization, in: Harvard Business Review, Vol. 66, 1988, S. 45 - 53.

Drury, C., Activity Based Costing, in: Management Accounting (UK), Vol. 67 , No. 8, 1989, S. 60 - 66.

Ellsberg, D., Risk, Ambiguity and the Savage Axioms, in: Quarterly Journal of Economics, Vol. 75, 1961, S. 643 - 669.

Emery, J. C., Management Information Systems, New York, Oxford, 1987.

Emery, J. C., Integrated Information Systems and their Effects on Organizational Structure, in: Information Systems and Organisational Structure, Hrsg.: Grochla, E., Szyperski, N., Berlin, New York, 1975, S. 95 - 103.

Emery, J.C., Organizational Planning and Control: Theory and Technology, London, 1969.

Farmer, D.H., MacMillan, K., Voluntary Collaboration vs. "Disloyalty" to suppliers, in: Journal of Purchasing in Materials Management, Vol. 12, No. 4, 1976, S. 3 - 8.

Farrell, J., Shapiro, C., Dynamic Competition with Lock-in, working paper, Department of Economics, University of California at Berkely, 1986.

Feder, B. J., Getting the Electronics Just Right, in: The New York Times, June 4, 1989, Section 3, S. 1 und 8.

Fink, R. B., Reevaluating ATM Strategy, in: United States Banker, Vol. 98, No. 4, April 1989, S. 60 - 63.

Fiol, C. M., Lyles, M. A., Organizational Learning, in: Academy of Management Review, Vol. 10, No. 4, 1985, S. $803-813$.

Fisher, A., Dresdner Confirms Allianz Holding, in: Financial Times, 25. Mai 1991, S. 10.

Foster, R., Innovation, New York, N. Y., 1986.

Francis, B., Frito Lays a New IS Bet, in: Datamation, Vol. 35, No. 4, 1989, S. $75-78$. 
Frank, R. H., Choosing the Right Pond, Human Behavior and the Quest for Status, New York, N. Y., 1985.

Franz, K.-P., Die Prozeßkostenrechnung im Vergleich mit der flexiblen Plankostenrechnung und der Deckungsbeitragsrechnung, in: Strategieunterstützung durch das Controlling: Revolution im Rechnungswesen?, Hrsg.: Horváth, P., Stuttgart, 1990, S. 195 - 210.

Frese, E., Grundlagen der Organisation, 4. Aufl., Wiesbaden, 1988.

Frese, E., Koordination, in: Handwörterbuch der Betriebswirtschaft, Hrsg.: Grochla, E., Wittmann, W., 4. Aufl., Stuttgart, 1975.

Galbraith, J. R., Designing Complex Organizations, Reading, Massachusetts u.a.O., 1973.

Galbraith, J. R., Achieving Integration Through Information Systems, in: Academy of Management Proceedings, 12, 1968, S. 111 - 120.

Galbraith, J. R., Kazanjian, R. K., Strategy Implementation. Structure, Systems, and Process, 2. ed., St. Paul u.a.O., 1986.

Gallupe, R.R., DeSanctis, G., Computer-Based Support for Group ProblemFinding: An Experimental Investigation, in: MIS Quarterly, Vol. 12, June 1988, S. $278-287$.

Gälweiler, A., Die strategische Führung der Unternehmung, in: Sonderdruck des Management-Zentrums St. Gallen 1980, S. 20.

Gälweiler, A., Unternehmenssicherung und strategische Planung, in: Zeitschrift für betriebswirtschaftliche Forschung, 28. Jg., 1976, S. 362 379.

Gälweiler, A., Unternehmensplanung, Grundlagen und Praxis, Frankfurt, New York, N. Y., 1974, S. 230.

Gentry, J.A., State of the Art of Short-Run Financial Management, Financial Management, Vol. 17, Summer 1988, S. 41 - 57.

Gershefski, G.W., Building a Corporate Financial Model, in: Harvard Business Review, Vol. 47, July-August 1969, S. 61 - 71.

Ghemawat, P., Commitment, The Dynamic of Strategy, Boston, 1991.

Ghemawat, P., Dauerhafte Wettbewerbsvorteile aufbauen, in: Wettbewerbsvorteile und Wettbewerbsfähigkeit, Hrsg.: Simon, H., Stuttgart, 1988 , S. 30 - 36.

Ghemawat, P., Sustainable Advantage, in: Harvard Business Review, Vol. 64,1986, S. 53 - 58. 
Gilbert, R. J., Mobility Barriers and the Value of Incumbency, in: Handbook of industrial organization, Hrsg.: Schmalensee, R., Willig, R., Amsterdam u.a.O., 1989, S. 476 - 535.

Gitomer, J., Seamless systems Integration, in: Journal of Information Systems Management, Vol. 6, Iss. 3, 1989, S. 77 - 80.

Gluck, F. W., Kaufmann, S. P., Walleck, A. S., Strategic Management for Competitive Advantage, in: McKinsey Quarterly, 1980.

Gluck, F. W., Strategic Choice and Resource Allocation, in: McKinsey Quarterly, Winter 1980, S. 22 - 33.

Goldberg, V.P., Toward an Expanded Economic Theory of Contract, in: Journal of Economic Issues, Vol. 10, 1976, S. 45 - 61.

Goldhar, J. D., Jelinek, M., Plan for Economies of Scope, in: Harvard Business Review, Vol. 61, November - December 1983, S. 141 - 148.

Gray, P. et. al., Assessing GDSS empirical research, Proceedings of the Conference on Information Systems and Decision Processes, 1989.

Grochla, E., Einführung in die Organisationstheorie, Stuttgart, 1978.

Grochla, E., Grundfragen der informationstechnologischen Integration, in: Seibt, D., Angewandte Informatik: Professor Dr. Paul Schmitz zu seinem 60. Geburtstag gewidmet, Braunschweig, 1985.

Grossman, S.J., Hart, O.D., The Costs and Benefits of Ownership: A Theory of Vertical and Lateral Integration, in: Journal of Political Economy, Vol. 94, 1986, S. 691 - 719.

Gutenberg, E., Einführung in die Betriebswirtschaftslehre, Bd. 1, Die Produktion, Berlin, Göttingen, Heidelberg, 1951.

Hage, J., Communication and Organizational Control, New York, N. Y., 1971.

Hahn, D., Strategische Unternehmensplanung. Ein konzentrierter Überblick, in: WISU, 10. Jg., Nr. 6, 1981, S. 275 - 279.

Hahn, D., Taylor, B., Hrsg., Strategische Unternehmensplanung, Stand und Entwicklungstendenzen, Heidelberg, Wien, 1986.

Hardin, G., The Tragedy of the Commons, in: Science, Vol. 162, No. 3859, December 13, 1968, S. 1243- 1248.

Hart, O.D., Incomplete Contracts and the Theory of the Firm, in: Journal of Law, Economics, and Organization, Vol.4, 1988, S. 119 - 139. 
Hauschildt, J., Zielsysteme, in: Handwörterbuch der Organisation, Hrsg.: Grochla, E., 2. Aufl., Stuttgart, 1980.

Hax, A., Majluf, N. Strategic Management. An Integrative Perspective, Englewood Cliffs, 1984.

Hax, H., Laux, H., Flexible Planung - Verfahrensregeln und Entscheidungsmodelle für die Planung bei Ungewißheit, in: Schmalenbachs Zeitschrift für betriebswirtschaftliche Forschung, 24. Jahrgang, 1972, S. 318 - 340 .

Hayek, Fr.A. von, The Use of Knowledge in Society, in: American Economic Review, Vol. 35, 1945, S. 519 - 530.

Heinen, E., Einführung in die Betriebswirtschaftslehre, 9. Aufl., Wiesbaden, 1986.

Heinen, E., Kostenlehre, Wiesbaden, 1983.

Heinen, E., Industriebetriebslehre als Entscheidungslehre, in: Industriebetriebslehre, Entscheidungen im Industriebetrieb, Hrsg.: Heinen, E., Wiesbaden, 1978.

Heinen, E., Grundlage betriebswirtschaftlicher Entscheidungen: Das Zielsystem der Unternehmung, Wiesbaden, 1976.

Heinen, E., Zum Wissenschaftsprogramm der entscheidungsorientierten Betriebswirtschaftslehre, in: Zeitschrift für Betriebswirtschaft, 1969, S. $207-220$.

Henderson, B., The Experience Curve Reviewed, IV. The Growth Share Matrix of the Product Portfolio, Perspectives, No. 135, Boston, MA:

Hinterhuber, H. H., Zur Objektivierung strategischer Entscheidungen, in: Informationstechnologie und strategische Führung, Hrsg.: Spremann, K., Zur, E., Wiesbaden 1989, S. 29 - 48.

Hinterhuber, H. H., Strategische Unternehmensführung, Berlin, New York, 1980.

Hirshman, A. O., Economic Development, Research and Policy Making: Some Converging Views, in: Behavioral Science, Vol. 7, April 1962, S. 211.

Hofman, D. J., Rockart, J. F., Lithonia Lighting: Case Study. Working Paper No. 201, Massachusetts Institute of Technology, Center for Information Systems Research, Cambridge, Massachusetts, 1989.

Holmstrom, B.R., Tirole, J., The Theory of the Firm, in: Handbook of Industrial Organization, Hrsg.: Schmalensee, R., Willig, R., Amsterdam, New York, 1989, S. 61 - 134. 
Hopper, M. D., Rattling SABRE - New Ways to Compete on Information, in: Harvard Business Review, Vol. 68, 1990, S. 118 - 125.

Horváth, P., Revolution im Rechnungswesen: Strategisches Kostenmanagement, in: Strategieunterstützung durch das Controlling: Revolution im Rechnungswesen?, Hrsg.: Horvath, P., Stuttgart, 1990.

Horváth, P., Hrsg., Strategieunterstützung durch das Controlling: Revolution im Rechnungswesen?, Stuttgart, 1990, S. 175 - 194.

Horváth, P., Meyer, R., Prozeßkostenrechnung, in: Controlling, Bd. 1, Nr. 4, 1989, S. $214-219$.

House, Ch. H., Price, R. L., The Return Map: Tracking Product Teams, in: Harvard Business Review, Vol. 81, January - February 1991, S. 92 100.

Huber, G.P., A Theory of the Effects of Advanced Information Technologies on Organizational Design, Intelligence, and Decision Making, Academy of Management Review, Vol. 15, 1990, S. 47 - 71.

Huber, G.P., Issues in the design of group decision support systems, MIS Quarterly, Vol. 8, 1984.

Hübner, H., Informationsmanagement. Strategie - Gestaltung - Instrumente, München, Wien, 1984.

Hübner, H., Integration und Informationstechnologie im Unternehmen, München, 1979.

Jaben, J., ATM Results Unproven, in: United States Banker, Vol. 99, No. 12, December 1988, S. 36- 41.

Janis, I., Victims of Groupthink, Boston, 1972.

Jarillo, C. J., Ricart, J.E.,Sustaining Networks, in: Interfaces, Vol. 17, No. 5, S. $82-91$.

Jeans, M., Morrow, M., The Practicalities of Using Activity-Based Costing, in: Management Accounting (UK), Vol. 67, No. 10, 1989, S. 42 - 44 .

Jensen, M.C., Meckling, W.H., Coordination, Control and the Management of Organizations, Vorlesungsskript, Boston, Massachusetts, Harvard Business School, 1988, S. 15.

Jensen, M.C., Meckling, W.H., Theory of the Firm: Managerial Behavior, Agency Costs, and Ownership Structure, in: Journal of Financial Economics, Vol. 3, No. 4, 1976, S. 305 - 360, wiederabgedruckt in: Barney, J.B., Ouchi, W.G., Organizational Economics, San Francisco, London, 1986, S. 214 - 275. 
Johansen, R., Groupware Computer Support for Business Teams, New York, N. Y., 1988.

Johansen, R., Teleconferencing and Beyond, New York, N. Y., 1984.

Johnson, H. Th., Activity-Based Information: A Blueprint for World Class Management Accounting, in: Management Accounting, Vol. 69, No. 12 , 1988, S. $23-30$.

Johnston, H. R., Lawrence, P. R., Beyond Vertical Integration - The Rise of the Value-Adding Partnership, in: Harvard Business Review, Vol. 66, July - August 1988, S. 94 - 101.

Johnston, H.R., Vitale, M.R., Creating Competitive Advantage with Interorganisational Information Systems, in: MIS Quarterly, June, Vol. 12, 1988, S. 153 - 165.

Jung, C. G., Integration of the personality, New York, N. Y., 1939.

Kanter, J., Management Information Systems, 3rd Ed., Englewood Cliffs, New Jersey, 1984.

Kaufmann, F., Data Systems That Cross Company Boundaries, in: Harvard Business Review, Vol. 44, January-February 1966, S. 141 - 149.

Keen, P.G.W., Competing in Time. Using Telecommunications for Competitive Advantage, Cambridge, Massachusetts, 1988.

Keen, P.G.W., Value Analysis: Justifying Decision Support Systems, in: MIS Quarterly, Vol. 5, March 1981, S. 1 - 14.

Keen, P.G.W., Scott Morton, M.S., Decision Support Systems, Reading, Massachusetts, 1978.

Keen, P.G.W., Wagner, G.R., DSS: An Executive Mind-Support System, in: Datamation, November, 1979, S. 117 - 122.

Keil, M., Sviokla, J.J., Du Pont's AI Task Force: The Water Intrusion Expert System, Boston, Massachusetts, Harvard Business School Case No. 9189-189, 1989.

Khandwalla, P. N., The design of organizations, New York, N. Y., 1977.

Kieser, A., Kubicek, H., Organisation, 2. Aufl., Berlin, 1983.

Kieser, A., Kubicek H., An Organizational Concept for the Design of Management Information Systems, in: Information Systems and Organisational Structure, Hrsg.: Grochla, E., Szyperski, N., Berlin, New York, 1975, S. 163 - 183. 
Kirsch, W., Picot, A., Hrsg., Die Betriebswirtschaftslehre im Spannungsfeld zwischen Generalisierung und Spezialisierung: Edmund Heinen zum 70. Geburtstag, Wiesbaden 1989.

Klein, B., Crawford, R., Alchian, A. A., Vertical Integration, Appropriable Rents, and the Competitive Contracting Process, in: Journal of Law and Economics, Vol. 21, 1978, S. 297 - 326.

Knight, F., Risk, Uncertainty, and Profit, Boston, 1921.

Koch, H., Integrierte Unternehmensplanung, Wiesbaden, 1982.

Kolodziej, S., The Glow of Success, in: Computerworld, Focus on Integration, October 2, 1989, S. 26 - 30.

Kolodziej, S., En Route to Customer Satisfaction, in: Computerworld, Focus on Integration, June 5, 1989, S. 43 - 46.

Konsynski, B. R., Mc Farlan, F. W., Information Partnerships - Shared Data, Shared Scale, in: Harvard Business Review, September October 1990, S. 114 - 120.

Konsynski, B., Vitale, M.R., Baxter Healthcare Corporation: ASAP Express, Boston, Massachusetts, Harvard Business School, Case No 9-188-080, revised July 9, 1990.

Koopmans, T., Three Essays on the State of Economic Science, New York, N. Y., 1957.

Korzeniowski, P., Bank Reaps Return on Net Investment, in: Network World, Vol. 4, No. 33, August 17, 1987, S. 1, 38.

Kosiol, E., Organisation der Unternehmung, 2. Aufl., Wiesbaden, 1976.

Kosiol, E., Aufbauorganisation, in: Handwörterbuch der Organisation, Hrsg.: Grochla, E., Stuttgart, 1969, Sp. 172 - 191.

Kosiol, E., Einführung in die Betriebswirtschaftslehre, Wiesbaden, 1968.

Kosiol, E., Organisation der Unternehmung, Wiesbaden, 1962.

Kotler, Ph., A Design for the Firm's Marketing Nerve Center, in: Business Horizons, Vol. 9, 1966, S. 70.

Kreps, D., Wilson, R., Reputation and Imperfect Information, in: Journal of Economic Theory, Vol. 27, 1982, S. 253 - 279.

Kroeber, D. W., Watson, H. J., Computer Based Information Systems, 2nd ed., New York, N. Y., 1987. 
Krüger, W., Pfeiffer, P., Eine konzeptionelle und empirische Analyse der Informationsstrategien und der Aufgaben des Informationsmanagements, in: Zeitschrift für betriebswirtschaftliche Forschung, 43. Jg., 1991, S. 21 - 43.

Kubicek, H., Informationstechnologie und organisatorische Regelungen, Berlin, 1975.

Laux, H., Grenzen integrativer Anreizsysteme, in: Der Integrationsgedanke in der Betriebswirtschaftslehre: Helmut Koch zum 70. Geburtstag, Hrsg.: Delfmann, W., Kuhn, Alfred, Wiesbaden, 1989, S. 201 - 218.

Laux, H., (Pareto-)Optimale Anreizsysteme bei unsicheren Erwartungen, in: Zeitschrift für Betriebswirtschaftliche Forschung, 40. Jg., 1988, S. 1093 - 1111.

Lawrence, P. R., Lorsch, J. W., Organization and Environment, Boston, Massachusetts, 1967.

Lax, D. A., Sebenius, J. K., The Manager as Negotiator, New York, London, 1986.

Lazarsfeld, P., Rosenberg, M., Hrsg., The Language of Social Research, Glencoe, Illinois, 1955.

Leavitt, Th., Whisler, Th. L., Management in the 1980's, in: Harvard Business Review, Vol. 36, 1958, S. 41 - 48.

Lee, P. M., The Micro-Marketing Revolution, in: Small Business Reports, Vol. 15, No. 2, 1990, S. 71 - 82.

Lehmann, H., Integration, in: Handwörterbuch der Organisation, Hrsg.: Grochla, E., 2. Aufl., Stuttgart, 1980.

Leibenstein, H., Beyond Economic Man, Cambridge, Massachusetts, 1976.

Leibenstein, H., Allocative Efficiency versus X-Efficiency, in: American Economic Review, Vol. 56, June 1966, S. 392 - 415.

Leonard-Barton, D., Sviokla, J.J., Putting Expert Systems to Work, in: Harvard Business Review, Vol. 66, 1988, S. 91 - 98.

Leontief, W., The Choice of Technology, in: Scientific American, Vol. 252, June 1985, S. 37 - 45.

Levitt, B., March, J. G., Organizational Learning, in: Annual Review of Sociology, Vol. 14, 1988, S. 319 - 340.

Linder, J. Mead, M., A Strategic Transition (A), Boston, Massachusetts, Harvard Business School Case No. 9-187-065, 1986. 
Loomes, G., Sugden, R., Regret Theory, in: Economic Journal, Vol. 92, 1982, S. 805 - 824.

Lowe, D., Cooperative Structuring of Information: The Representation of Reasoning and Debate, Journal of Man-Machine Studies, Vol. 23, 1985, S. $97-111$.

Luce, R. D., Raiffa, H., Games and Decisions, Paperback Reprint, New York, N. Y., 1957.

MacMillan, K., Farmer, D., Redefining the Boundaries of the Firm, in: Journal of Industrial Economics, Vol. 27, No. 3 , 1979, S. 277 - 285.

Main , T., Computers of the World, Unite!, in: Fortune, Vol. 122, No. 7, 1990 , S. $113-122$.

Main, T.J., Short, J. E., Managing the Merger: Building Partnership Through IT Planning at the New Baxter, in: MIS Quarterly, Vol. 13, December 1989, S. 469 - 484.

Malone, Th. W., Yates, J., Benjamin, R. I., The Logic of Electronic Markets, in: Harvard Business Review, Vol. 67, May - June 1989, S. 166 - 170.

Malone, Th. W., Yates, J., Benjamin, R. I., Electronic Markets and Electronic Hierarchies, in: Communications of the ACM, Vol. 30, June 1987, S. 484 - 497.

March, J. G., Simon, H. A., Organizations, New York, N. Y., 1958.

McCaskey, M. B., The executive challenge: managing change and ambiguity, Boston, 1982.

McFarlan, F.W., Bruns, W., How Information Technology Is Changing Management Control Systems, Boston, Massachusetts, Harvard Business School Note \# 9-187-139, 1987.

McFarlan, F.W., Information Technology Changes the Way You Compete, Harvard Business Review, Vol. 62, May-June, 1984, S. 98-103.

McGee, J. V., Vitale, M. R., Consolidated Rail Corporation: Railcar Identification Project, Boston, Massachusetts, Harvard Business School Case No. 9-188-082, Revised Version, 5/88.

McGee, J.V., Konsynski, B., Transnet, Boston, Massachusetts, Harvard Business School Case No. 9-188-098, 1988.

McGraw-Hill, Product Support Architecture, New York, N. Y., 1985.

McKenney, J. L., McFarlan, F. W., The Information Archipelago - Maps and Bridges, in: Harvard Business Review, Vol. 60, No. 5, 1982. 
McLeod, R. Jr., Management Information Systems. A Study of Computer Based Information Systems, 4. Auflage, New York, N. Y., 1990.

Mead, M., ESAB AB: Toughing it Out (A), Boston, Massachusetts, Harvard Business School, Case No. 9-188-005, 1988.

Meffert, H., Klassische Funktionenlehre und marktorientierte Führung Integrationsperspektiven aus der Sicht des Marketing, - S. 372 - 408 in: Integration und Flexibilität, Hrsg.: Albach, H. u.a., Wiesbaden 1990.

Meffert, H., Die Wertkette als Instrument einer integrierten Unternehmensplanung, in: Der Integrationsgedanke in der Betriebswirtschaftslehre, Hrsg.: Delfman, W., Kuhn, A., Wiesbaden, 1989, S. 255 - 278.

Meffert, H., Strategische Unternehmensführung und Marketing, Wiesbaden, 1988.

Meffert, H., Marketing, Grundlagen der Absatzpolitik, 7. Aufl., Wiesbaden, 1987

Meffert, H., Marketing und strategische Unternehmensführung - ein wettbewerbsorientierter Kontingenzansatz, in: Strategische Unternehmensplanung, Stand und Entwicklungstendenzen, Hrsg.: Hahn, D., Taylor, B., Heidelberg , Wien, 1986, S. 660 - 683.

Meffert, H., Größere Flexibilität als Unternehmenskonzept, in: Zeitschrift für betriebswirtschaftliche Forschung, Vol. 37, 1985, S. 121 - 137.

Meffert, H., Computergestützte Marketing-Informationssysteme, Konzeptionen, Modellanwendungen, Entwicklungsstrategien, Wiesbaden 1975.

Meffert, H., Informationssysteme. Grundbegriffe der EDV und Systemanalyse, Tübingen, Düsseldorf, 1975.

Meffert, H., Katz, R., Unternehmensverhalten in stagnierenden und gesättigten Märkten: Ergebnisse einer empirischen Untersuchung in der Bundesrepublik Deutschland, Wissenschaftliche Gesellschaft für Marketing und Unternehmensführung, Münster, 1983.

Meffert, H., Ohlson, G., Was Sie beim Marktein- und -austritt beachten müssen, in: Die Absatzwirtschaft 1982, Nr. 10, Sonderausgabe, 1982, S. $178-190$.

Mellerowicz, K., Unternehmenspolitik, Bd. 1, Freiburg i. Br., 1963.

Mertens, P., Griese, J., Industrielle Datenverarbeitung II, 2. Auflage, Wiesbaden, 1979. 
Mertens, P., Plattfaut, E., Ansätze zur DV-Unterstützung der strategischen Unternehmensplanung, in: Die Betriebswirtschaft, Vol. 43, 1985, S. 19 -30 .

Mertens, P., Plattfaut, E., Informationstechnik als strategische Waffe, in: Information Management, Nr. 2, 1986, S. 6 - 17.

Mertens, P., Schumann, M. Hohe, U., Informationstechnik als Mittel zur Verbesserung der Wettbewerbsposition - Erkenntnisse aus einer Beispielsammlung, in: Informationstechnologie und strategische Führung, Hrsg.: Spremann, K., Zur, E., Wiesbaden, 1989, S. 109 - 136.

Mesarovic, M. D., Macko, D., Takahara, Y., Theory of Hierarchical, Multilevel Systems, New York, London, 1970.

Meyer, J., Qualität als strategische Wettbewerbswaffe, in: Wettbewerbsvorteile und Wettbewerbsfähigkeit, Hrsg.: Simon, H., Stuttgart, 1988, S. $73-88$.

Meyer, N. D., Boone, M. E., The Information Edge, New York, N. Y., 1987.

Miles, R. E., Snow, Ch. C., Network Organizations - New Concepts for New Forms, in: McKinsey Quarterly, Autumn, 1986, S. 53 - 66.

Miles, R.E., Snow, Ch., Fit, Failure and the Hall of Fame, in: California Management Review, Vol. 26, No. 3, 1984, S. 10 - 28.

Milgrom, P., Employment Contracts, Influence Activities, and Efficient Organizational Design, in: Journal of Political Economy, Vol. 96, 1988, S. $42-60$.

Mills, R.G., Reponen, T., McFarlan, F. W., Finnpap/Finnboard (B) FinProject, Boston, Massachusetts, Harvard Business School Case No. 9188-103, Revised July 1988.

Mintzberg, H., The Structuring of Organizations, Englewood Cliffs, New Jersey, 1979.

Mintzberg, H., Patterns in Strategy Formation, in: Management Science, Vol. 24, 1978 S. 934-948.

Mises, L. von, Human Action, 3. Nachdruck, Chicago, 1949.

Nagel, E., On the statement: "The whole is more than the sum of its parts", in: The language of social research, Hrsg.: Lazarsfeld, P., Rosenberg, M., Glencoe, Illinois, 1955, wiederabgedruckt als: Über die Aussage: "Das Ganze ist mehr als die Summe seiner Teile", in: Logik der Sozialwissenschaften, Hrsg:, Topitsch, F. von, Köln, Berlin, 1965, S. 225-235. 
Nagel, K., Bewertung strategischer Wettbewerbsvorteile durch Informationssysteme, in: Spremann, K., Zur, E., Informationstechnologie und strategische Führung, Wiesbaden, 1989, S. 49 - 64.

Nicklisch, H, Organisation, 2. Aufl., Stuttgart 1922.

Ordelheide, D., Rudolph, B., Büsselmann, E., Hrsg., Betriebswirtschaftslehre und ökonomische Theorie, Jahrestagung des Verbandes der Hochschullehrer für Betriebswirtschaft vom 6. Juni 1990, Stuttgart, 1991.

Osborn, T. W., Micro Marketing into the Niches: The Eye of the Stranger, in: Marketing Communications, Vol. 12, No. 3, 1987, S. 57 - 72.

Ostrofsky, K., Mrs. Fields Cookies, Boston, Massachusetts, Harvard Business School Case No. 9-189-056, Rev. 25-07-89, 1989.

Overlack, J., Wettbewerbsvorteile durch Informationstech-nologie, Frankfurt a. M. u.a.O., 1988.

o.V., Gaining the Competitive Edge, in: Fleet Owner, Big Fleet Edition, Vol. 85, No. 5, 1990, S. 82 - 86.

o.V., The LeBaron Phenomenon, in: Institutional Investor, August, 1985, S. $104-115$.

o.V., Ordering System Dries Up Flow of Paperwork, in: Modern Healthcare, May 1982, S. 147.

Panzar, J. C., Determinants of Firm and Industry Structure, in: Handbook of industrial organization, Hrsg.: Schmalensee, R., Willig, R., Amsterdam u.a.O., 1989.

Parsons, G. L., Information Technology: A New Competitive Weapon, in: Sloan Management Review, Vol. 25, Fall, 1983, S. 3 - 13.

Parsons, G. L., Strategic Information Technology, Boston, Massachusetts, Harvard Business School Paper, 1983.

Perrow, C. A., Framework for Comparative Organizational Analysis, American Sociological Review, 1967, Vol. 32, S. 194 - 208

Peters, T., The Destruction of Hierarchy, in: Industry Week, August 15, 1988, S. 33 - 35.

Peters, T./Waterman, J.,In Search of Excellence, New York, N. Y. 1982 S. 8 $-19$ 
Picot, A., Ökonomische Theorien der Organisation - Ein Überblick über neuere Ansätze und deren betriebswirtschaftliches Anwendungspotential, in: Betriebswirtschaftslehre und ökonomische Theorie, Jahrestagung des Verbandes der Hochschullehrer für Betriebswirtschaft vom 6. Juni 1990, Hrsg.: Ordelheide, D., Rudolph, B., Büsselmann, E., Stuttgart, 1991, S. 143 - 170.

Picot, A., Zur Bedeutung allgemeiner Theorieansätze für die betriebswirtschaftliche Information und Kommunikation: Der Beitrag der Transaktionskosten- und Principal-Agent-Theorie, in: Die Betriebswirtschaftslehre im Spannungsfeld zwischen Generalisierung und Spezialisierung: Edmund Heinen zum 70. Geburtstag, Hrsg.: Kirsch, W., Picot, A., Wiesbaden, 1989, S. 363 - 379.

Picot, A., Kommunikationstechnik und Dezentralisierung, in: Information und Wirtschaftlichkeit, Hrsg.: Ballwieser, W., Berger, K. H., Wiesbaden, 1985.

Porter, M. E., Competitive Advantage, New York, N. Y., 1985.

Porter, M. E., Competitive Strategy, New York, N. Y., 1980.

Porter, M. E., Millar, V. E., How information gives you competitive advantage, in: Harvard Business Review, Vol. 63, No. 4, 1985.

Pratt, J.W., Zeckhauser, R.J., Hrsg., Principals and Agents: The Structure of Business, Boston, Massachusetts, 1985.

Remmerbach, K.-U., Markteintrittsentscheidungen, Wiesbaden, 1988.

Reponen, T., Copeland, D. McFarlan, F.W., Finnpap/Finnboard (A), Boston, Massachusetts, Harvard Business School Case No. 9-186-130, 1985.

Riebel, P., Produktion III: einfache und verbundene, in: HdWW, Band 6, Hrsg.: Albers, W. u.a., Stuttgart, 1981, S. 295 - 310.

Roberts, E. B., Managing Invention and Innovation, in: Research Technology Management, Vol. 31, No. 1, 1988, S. 11 - 29.

Rockart, J. F., De Long, D., Executive Support Systems, Homewood, Illinois, 1988.

Rockart, J. F., Short, J. E., IT in the 1990's: Managing Organizational Interdependence, in: Sloan Management Review, Vol. 30, Winter 1989, S. 7 - 17.

Rockart, J.F., Short, J.E. IT and the Networked Organization: Towards More Effective Management of Interdependence, Working Paper, Center for Information Systems Research, MIT, 1989. 
Rockart, J. F., Short, J. E., Information Technology and the New Organization: Towards More Effective Management of Interdependence, Working Paper No. 180, Massachusetts Institute of Technology, Center for Information Systems Research, Cambridge, Massachusetts, 1988.

Rockart, J.F., Treacy, M.E., The CEO goes On-Line, in : Harvard Business Review, January-February 1982, S. 82 - 88.

Ropella, W., Synergie als strategisches Ziel der Unternehmung Berlin, New York, 1989.

Rothfeder, J., Bartimo, J., How Software is Making Food Sales a Piece of Cake, in: Business Week, 2. July 1990, S. 54, 55.

Sannwald, R., Wirtschaftliche Integration, Basel, 1958.

Sauter, E., Making the Right Connections, in: World, Vol. 21, No. 3, Jul Sep 1987, S. $36-43$.

Schanz, G., Organisationsgestaltung, München, 1982.

Scheer, A.-W., EDV-orientierte Betriebswirtschaftslehre, 4. Aufl., Berlin u.a.O., 1990.

Schelling, T.C., The Strategy of Conflict, Boston, Massachusetts, 1960.

Schiller, Z., Stalking the New Consumer, in: Business Week, 26. August 1989, S. $54-62$.

Schlick, M., Gesammelte Aufsätze, Wien, 1938.

Schlick, M., Über den Begriff der Ganzheit, in: Moritz Schlick, Gesammelte Aufsätze, Wien, 1938 wiederabgedruckt in: Logik der Sozialwissenschaften, Hrsg.: Topitsch, F. von, Köln, Berlin, 1965, S. 213 - 224.

Schmalenbach, E., Die Betriebswirtschaftslehre an der Schwelle der neuen Wirtschaftsverfassung, in: Zeitschrift für handelswissenschaftliche Forschung, 22. Jg., 1928, S. 241 - 251.

Schmalensee, R., Willig, R., Hrsg., Handbook of Industrial Organization, Amsterdam, New York, 1989.

Schmerken, I., Expert Systems Churns Many Happy Returns, in: Wall Street Computer Review, Vol. 5, No. 3, December 1987, S. 14 - 17.

Schmitzer, I. L., ATM's at Maturity, in: Texas Banking, Vol. 77, No. 12, December, 1988, S. 11, 12.

Scholz, Chr., Strategisches Management, Berlin, New York, 1987. 
Schumann, J., Grundzüge der mikroökonomischen Theorie, 3. Auflage, Berlin, Heidelberg, New York 1980.

Schweiker, K., Grundlagen einer Theorie betrieblicher Datenverarbeitung, Wiesbaden, 1966.

Scott Morton, M.S., The Corporation of the 1990s - Information Technology and Organizational Transformation, New York, Oxford 1991.

Seibt D., Angewandte Informatik: Professor Dr. Paul Schmitz zu seinem 60. Geburtstag gewidmet, Braunschweig, 1985.

Shank, J. K., Strategic Cost Management - New Wine, or just New Bottles?, in: Journal of Management Accounting Research, Vol. 1, Fall, 1989, S. 47 - 65.

Shingo, Sh., [Shinguru dandori.] English: A revolution in manufacturing: the SMED system. Stamford, Connecticut, 1985.

Siegwarth, H., Overlack, J., Langfristiger Erfolg durch Qualitätsstrategien, in: Harvard Manager, Heft 3, 1986, S. 64 - 69.

Silber, K., Facing a New ATM Reality, in: Bank Systems \& Technology, Vol. 26, No. 12, December 1989, S. 26 - 30.

Siler, F., Will Another Round of Surgery help Baxter?, in: Business Week, 30. April 1990, S. 92.

Simon, H., Hrsg., Wettbewerbsvorteile und Wettbewerbsfähigkeit, Stuttgart, 1988.

Simon, H. A., Administrative Behavior, New York, N. Y., 1976.

Simon, H.A., The New Science of Management Decision, New York, N. Y., 1960.

Simon, H.A., Models of Man: New York, N. Y., 1957.

Simson, C. von, Food Fight, in: Computerworld, Focus on Integration, 2. October 1989, S. 22 - 25.

Smith, H. J., Applegate, L. M., IBM Computer Conferencing, Boston, Massachusetts, Harvard Business School Case No. 9-188-039, 1988.

Snow, C. C., Hrsg., Strategy, Organisation Design and Human Resource Management, Greenwich, Conneticut, London, England, 1989.

Spencer, H., System of Synthetic Philosophy, Aberdeen, 1893. 
Spragins, E.E., Aikman, R., American Hospital Supply: Snaring New Business with Freebies and Bonuses, in: Business Week, April 8, 1985, S. 88 und 89.

Sprague, R.H., Carlson, E.D., Building Effective Decision Support Systems, Englewood Cliffs, New Jersey, 1982.

Spremann, K., Zur, E., Informationstechnologie und strategische Führung, Wiesbaden, 1989.

Staehle, W., Deutschsprachige situative Ansätze in der Managementlehre, in: Wirtschaftswissenschaftliches Studium, 1979, S. 218 - 222.

Stalk, G., Time - The Next Source of Competitive Advantage, in: Harvard Business Review, Vol. 66. No. 4, S. 41 - 51.

Stalk, G., Hout, Th. M., Competing Against Time, New York, London, 1990.

Stalk, G., The Strategic Value of Time, in: Time-Based Competition, Hrsg:: Blackburn, J. D., Homewood, llinois, 1991.

Stoddard, D., McFarlan, F. W., Otisline, Boston, Massachusetts, Harvard Business School Case No. 9-186-304, 1986.

Szyperski, N., Führungstechnische Integration eines differenzierten Informations- und Kommunikationsmanagements, in: Seibt, D., Angewandte Informatik, Braunschweig, 1985, S. 15 - 28.

Szyperski, N., Informationssysteme, computergestützte. In: Handwörterbuch der Organisation, Hrsg.: Grochla, E., 2. Aufl., Stuttgart, 1980, Sp. 1900 - 1910.

Szyperski, N., Winand, U., Informationsmanagement und informationstechnische Perspektiven, in: Seidel, E., Wagner, D. Hrsg., Organisation, Festschrift für Knut Bleicher, Wiesbaden, 1989.

Teece, D. J., Economies of Scope and the Scope of the Enterprise, in: Journal of Economic Behavior and Organization, Vol. 1, 1980, S. 223 247.

Thompson, J. D., Organizations in Action, New York, N. Y., 1967.

Thorelli, H. B., Networks, Between Markets and Hierarchies, in: Strategic Management Journal, Vol. 7, No. 1, 1986, S. 37 - 51.

Topitsch, F. von, Hrsg., Logik der Sozialwissenschaften, Köln, Berlin, 1965.

Turney, P. B., Anderson, B., Accounting for Continous Improvement, in: Sloan Management Review, Vol. 30, No. 2, 1989, S. 37 - 47. 
Ulrich, H., Integrative Unternehmensführung, in: Kirsch, W., Picot, A., Die Betriebswirtschaftslehre im Spannungsfeld zwischen Generalisierung und Spezialisierung, Wiesbaden, 1989, S. 184 - 198.

United States Civil Aeronautics Board, Report to Congress on Airline Computer Reservations Systems, 1983.

Van de Ven, A., Delbecq, A., Koenig, R. Jr., Determinants of Coordination Modes Within Organizations, in: American Sociological Review, Vol. 41, 1976, S. 322 - 338.

Venkatraman, N., Strategic Management and Information Technology: Evolutionary Linkages and a Research Framework, in: Strategy, Organisation Design and Human Resource Management, Hrsg.: Snow, C. C., Greenwich, Conneticut, London, England, 1989.

Venkatraman, N., Kambil, A., The Check's Not in the Mail: Strategies for Electronic Integration Tax Return Filing, Sloan Management Review, Vol. 32, Winter 1991, S. 34 - 43.

Venkatraman, N., Short, J.E., Strategies for Electronic Integration: From Order-Entry to Value-Added Partnerships at Baxter, Author's Draft, Massachusetts Institute of Technology, Cambridge, Massachussetts, 1990.

Venkatraman, N., Zaheer, A., Electronic Integration and Strategic Advantage: A Quasi-Experimental Study in the Insurance Industry, International Conference on Information Systems, Boston, Massachusetts, December 4-6, 1989.

Vieweg, H.-G., Koordination: die Entwicklung eines Modells und seine Anwendung in einer empirischen Untersuchung, München, 1987.

Vitale, M.R., American Hospital Supply Corp.: The ASAP System (A) \& (B), MIS Teaching Note, Boston, Massachusetts, Harvard Business School, Case No 5-188-119, revised April, 1989, S. 7.

Vitale, M. R., Benetton S.p.A.: Industrial Fashion (A), Boston, Massachusetts, 1988.

Vitale, M.R., American Hospital Supply Corp.: The ASAP System (A) \& (B), Boston, Massachusetts, Harvard Business School, Cases No 9186-005 und 9-186-006, 1986.

Vitale, M. R., Earl, H., British Home Supply (BhS), Boston, Massachusetts, 1988.

Vitale, M.R., Ives, B., MBS Textbook Exchange, Boston, Massachusetts, Harvard Business School Case No. 9-188-028, 1988. 
Vogel D., Nunamaker J., Applegate L., and Konsynski B., Group Decision Support Systems: Determinants of Success, DSS-87 Transactions, 7th International Conference on Decision Support Systems, 1987, S. 118 128.

Walton, R. E., Up and Running, Integrating Information Technology and the Organization, Boston, Massachusetts, 1989.

Warbelow, A., Kokuryo, J., Konsynski, B., Aucnet TV Auction Network System, Boston, Massachusetts, Harvard Business School Case No. 9190-001, Rev. 19.7.89, 1989.

Warbelow, A., Konsynksi, B., American Gem Market System, Boston, Massachusetts, Harvard Business School Case No. 9-189-088, 1988.

Warbelow, A., Konsynksi, B., IVANS, Boston, Massachusetts, Harvard Business School Case No. 9-187-188, Revised July 1987.

Webber, J. C., Packaged Goods Marketing Research - Where's It All Going, in Journal of Advertizing Research, Vol. 26, No. 5, S. RC3 - RC5.

Weick, K. E., The Social Psychology of Organizing, 2nd ed., New York, N. Y., 1979.

Welge, M.K., Unternehmensführung, Band 2: Organisation; unter Mitwirkung von Herbert Kubicek, Stuttgart, 1986.

Whisler, Th. L., Information Systems and Organisational Structure, Berlin, New York, 1975.

Whisler, Th. L., Man, Organization, and Computer - A Contingency Analysis, in: in: Information Systems and Organisational Structure, Hrsg.: Grochla, E., Szyperski, N., Berlin, New York, 1975, S. 246 - 267.

Whisler, Th. L., Information Technology and Organizational Change, Belmont, California, 1970.

Wildemann, H., Erfolgspotentialaufbau durch neue Produktionstechnologien, in: Wettbewerbsvorteile und Wettbewerbsfähigkeit, Hrsg.: Simon, H., Stuttgart, 1988.

Williamson, O. E., Comparative Economic Organization - Vergleichende ökonomische Organisationstheorie: Die Analyse diskreter Strukturalternativen, in: Ordelheide, D., Rudolph, B., Büsselmann, E., Hrsg., Betriebswirtschaftslehre und ökonomische Theorie, Stuttgart, 1991, S. 13 - 49.

Williamson, O. E., The Economic Institutions of Capitalism, New York, N. Y., 1985. 
Williamson, O. E., Transaction Cost Economics: The Governance of Contractual Relations, in: Journal of Law and Economics, Vol. 22, 1979, S. 3 - 61.

Williamson, O.E., Markets and Hierarchies, Analysis and Antitrust Applications, New York, N. Y., 1975.

Williamson, O. E., The Economics of Discretionary Behavior: Managerial Objectives in the Theory of the Firm, Englewood Cliffs, New Jersey, 1964.

Wiseman, C., Attack \& Counterattack: The New Game in Intormation Technology, in: Planning Review, Vol. 16, No. 5, September/October, 1988, S. $6-12$.

Wiseman, Ch., Strategy and Computers, Homewood, Illinois, 1985.

Wittek, B., Strategien auf Kundennutzen aufbauen, in: Wettbewerbsvorteile und Wettbewerbsfähigkeit, Hrsg.: Simon, H., Stuttgart, 1988, S. $66-72$.

Wöhe, G., Einführung in die Allgemeine Betriebswirtschaftslehre, 14. Aufl., München, 1981.

Wouw, C., Information Systems Planning, Eindhoven, 1977.

Yates, J., Benjamin, R. The Past and the Present as a Window on the Future, in: The Corporation of the 1990s - Information Technology and Organizational Transformation, Hrsg:: Scott Morton, M.S., New York, Oxford 1991, S. 61 - 94

Zimmer, L. F., Reviving ATMs, in: Bank Management, Vol. 66, Iss. 6, Juni 1990, S. 58 - 61, 64.

Zottola, L., The United Systems of Benetton, in: Computerworld, Vol. 24, No. 14, 1990, S. 70.

Zuboff, S., In the Age of the Smart Machine, New York, N. Y., 1988. 
Nicolaus Henke - 978-3-631-75100-8

Downloaded from PubFactory at 01/11/2019 08:21:33AM

via free access 


\section{SCHRIFTEN ZUM MARKETING}

Band 1 Friedrich Wehrle: Strategische Marketingplanung in Warenhăusern. Anwendung der Portfolio-Methode. 1981. 2. Auflage. 1984.

Band 2 Jürgen Althans: Die Übertragbarkeit von Werbekonzeptionen auf internationale Mărkte. Analyse und Exploration auf der Grundlage einer Befragung bei europaweit tátigen Werbeagenturen. 1982.

Band 3 Günter Kimmeskamp: Die Rollenbeurteilung von Handelsvertretungen. Eine empirische Untersuchung zur Einschătzung des Dienstleistungsangebotes durch Industrie und Handel. 1982.

Band 4 Manfred Bruhn: Konsumentenzufriedenheit und Beschwerden. Erklărungsansătze und Ergebnisse einer empirischen Untersuchung in ausgewăhtten Konsumbereichen. 1982.

Band 5 Heribert Meffert (Hrsg.): Kundendienst-Management. Entwicklungsstand und Entscheidungsprobleme der Kundendienstpolitik. 1982.

Band 6 Ralf Becker: Die Beurteilung von Handelsvertretem und Reisenden durch Hersteller und Kunden. Eine empirische Untersuchung zum Vergleich der Funktionen und Leistungen. 1982.

Band 7 Gerd Schnetkamp: Einstellungen und Involvement als Bestimmungsfaktoren des sozialen Verhaltens. Eine empirische Analyse am Beispiel der Organspendebereitschaft in der Bundesrepublik Deutschland. 1982.

Band 8 Stephan Bentz: Kennzahlensysteme zur Erfolgskontrolle des Verkaufs und der MarketingLogistik. Entwicklung und Anwendung in der Konsumgüterindustrie. 1983.

Band 9 Jan Honsel: Das Kautverhalten im Antiquitătenmarkt. Eine empirische Analyse der Kaufmotive, ihrer Bestimmungsfaktoren und Verhaltenswirkungen. 1984.

\section{SCHAIFTEN ZU MARKETING UND MANAGEMENT}

Band 10 Matthias Krups: Marketing innovativer Dienstleistungen am Beispiel elektronischer Wirtschaftsinformationsdienste. 1985.

Band 11 Bernd Faehsler: Emotionale Grundhaltungen als Einflußfaktoren des Kăuferverhaltens. Eine empirische Analyse der Beziehungen zwischen emotionalen Grundhaltungen und ausgewăhlten Konsumstrukturen. 1986.

Band 12 Ernst-Otto Thiesing: Strategische Marketingplanung in filialisierten Universalbanken. Integrierte Filial- und Kundengruppenstrategien auf der Grundlage erfolgsbeeinflussender Schlüsselfaktoren. 1986.

Band 13 Rainer Landwehr: Standardisierung der internationalen Werbeplanung. Eine Untersuchung der Prozeßstandardisierung am Beispiel der Werbebudgetierung im Automobilmarkt. 1988.

Band 14 Paul-Josef Patt: Strategische Erfolgsfaktoren im Einzelhandel. Eine empirische Analyse am Beispiel des Bekleidungsfachhandels. 1988. 2. Auflage. 1990.

Band 15 Elisabeth Tolle: Der Einfluß ablenkender Tătigkeiten auf die Werbewirkung. Bestimmungsfaktoren der Art und Hőhe von Ablenkungseffekten bei Rundfunkspots. 1988.

Band 16 Hanns Ostmeier: Ökologieorientierte Produktinnovationen. Eine empirische Analyse unter besonderer Berücksichtigung ihrer Erfolgseinschătzung. 1990.

Band 17 Bernd Büker: Qualitătsbeurteilung investiver Dienstleistungen. Operationalisierungsansătze an einem empirischen Beispiel zentraler EDV-Dienste. 1991.

Band 18 Kerstin Ch. Monhemius: Umweltbewußtes Kautverhalten von Konsumenten. Ein Beitrag zur Operationalisierung, Erklărung und Typologie des Verhaltens in der Kaufsituation. 1993. 
Band 19 Uwe Schürmann: Erfolgsfaktoren der Werbung im Produktlebenszyklus. Ein Beitrag zur Werbewirkungsforschung. 1993.

Band 20 Ralf Birkelbach: Qualitătsmanagement in Dienstleistungscentern. Konzeption und typenspezifische Ausgestaltung unter besonderer Berücksichtigung von Verkehrsflughăfen. 1993.

Band 21 Simone Frömbling. Zielgruppenmarketing im Fremdenverkehr von Regionen. Ein Beitrag zur Marktsegmentierung auf der Grundlage von Werten, Motiven und Einstellungen. 1993.

Band 22 Marcus Poggenpohl: Verbundanalyse im Einzelhandel auf der Grundlage von Kundenkarteninformationen. Eine empirische Untersuchung von Verbundbeziehungen zwischen Abteilungen. 1994.

Band 23 Kai Bauche: Segmentierung von Kundendienstleistungen aưf investiven Mărkten. Dargestelt am Beispiel von Personal Computem. 1994.

Band 24 Ewald Werthmöller: Răumliche Identităt als Aufgabenfeld des Stădte- und Regionenmarketing. Ein Beitrag zur Fundierung des Placemarketing. 1995.

Band 25 Nicolaus Müller: Marketingstrategien in High-Tech-Mărkten. Typologisienung, Ausgestaltungsformen und Einflußfaktoren auf der Grundlage strategischer Gruppen. 1995.

Band 26 Nicolaus Henke: Wettbewerbsvorteile durch Integration von Geschăftsaktivităten. Ein zeitablaurbezogener wettbewerbsstrategischer Analyseansatz unter besonderer Berücksichtigung des Einsatzes von Kommunikations- und Informationssystemen (KIS). 1995. 


\title{
Rainer Bieker
}

\section{Entwicklung von Wettbewerbsvorteilen für Technologie-Unternehmen \\ - untersucht am Beispiel der Unternehmensneugründung -}

\author{
Frankfurt/M., Bern, New York, Paris, 1990. V, 297 S., Abb. u. Tab. \\ Europäische Hochschulschriften: Reihe 5, Volks- und Betriebswirtschaft. Bd. 1057 \\ ISBN 3-631-42325-X br. DM 86.--*
}

Mit der vorliegenden Arbeit wird neugegründeten, technologieorientierten Unternehmen ein Instrumentarium zur Verfügung gestellt, das ihnen helfen soll, sich optimal zu entwickeln. Die Untersuchung baut auf Erfahrungen des Autors mit einer Vielzahl von High-Tech-Unternehmen auf, die er in vierjähriger Arbeit bei einer der führenden deutschen Venture-Management und Gründungsberatungsgesellschaften sammelte. Es wird systematisch analysiert, welche Ansatzpunkte sich einem High-Tech-Unternehmen bieten, Wettbewerbsvorteile aufzubauen und zu verteidigen. Anschließend werden für verschiedene Marktsituationen Strategien entwickelt, die es einem Unternehmen gestatten, seine Wettbewerbsvorteile effizient umzusetzen, um sich im Markt zu behaupten. Die vorliegende Untersuchung gehört zu den wenigen, die sich speziell mit Marketing-Strategien für High-Tech-Unternehmen beschäftigen. Hervorzuheben ist, daß sie besonders auf die Spezifika neu gegründeter Unternehmen eingeht. Sie richtet sich gleichermaßen an Wissenschaftler und Praktiker.

Aus dem Inhalt: Marketing-Strategien für neu gegründete High-TechUnternehmen

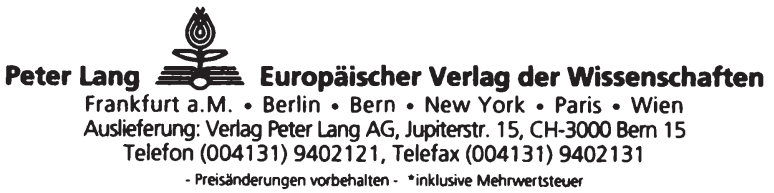


Nicolaus Henke - 978-3-631-75100-8

Downloaded from PubFactory at 01/11/2019 08:21:33AM

via free access 